\title{
\#USES
}

science for a changing world

Prepared in cooperation with the

JOHNSON COUNTY STORMWATER MANAGEMENT PROGRAM

\section{Effects of Nonpoint and Selected Point Contaminant Sources on Stream-Water Quality and Relation to Land Use in Johnson County, Northeastern Kansas, October 2002 Through June 2004}

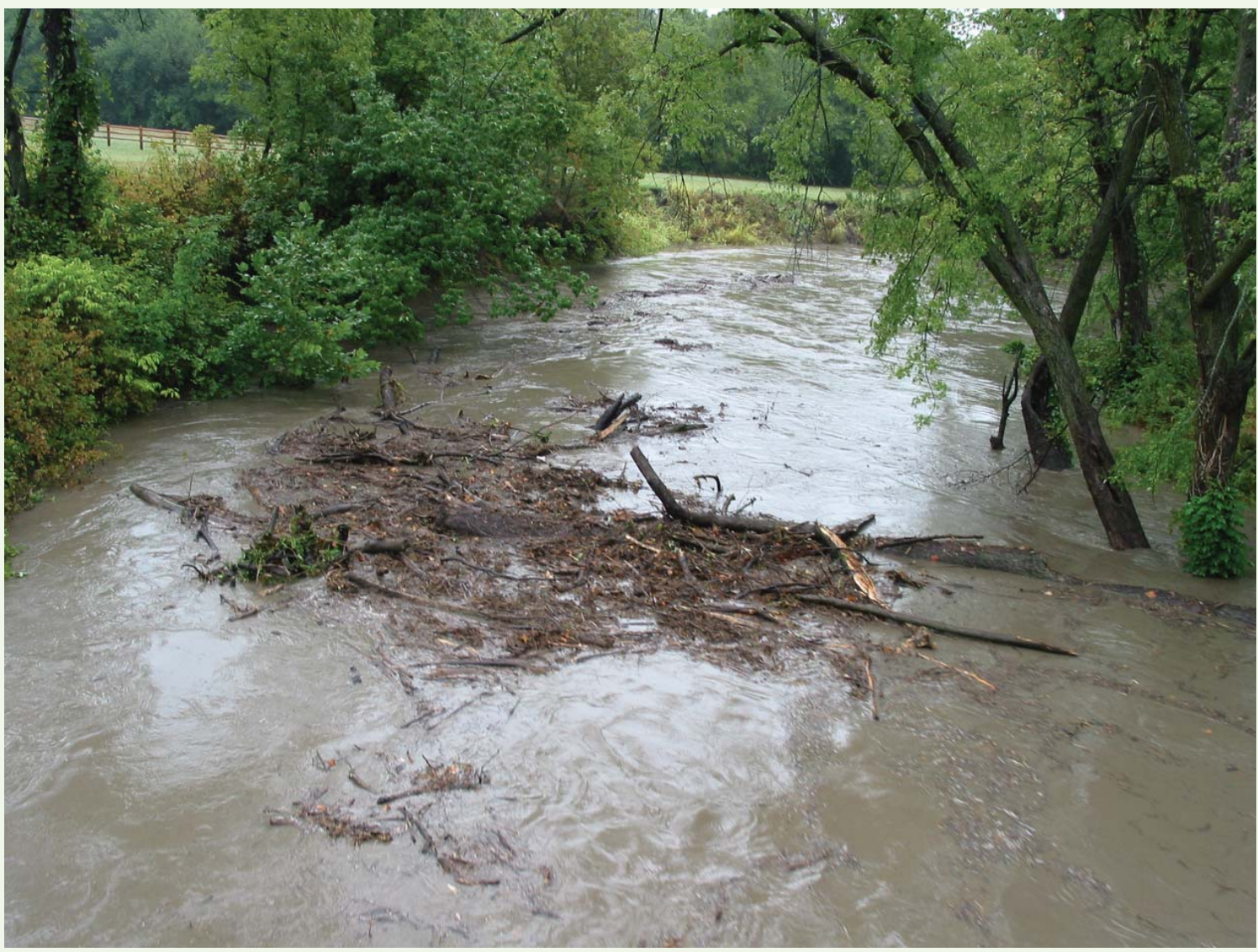

Scientific Investigations Report 2005-5144 


\section{Effects of Nonpoint and Selected Point Contaminant Sources on Stream-Water Quality and Relation to Land Use in Johnson County, Northeastern Kansas, October 2002 Through June 2004}

By Casey J. Lee, D.P. Mau, and T.J. Rasmussen

Scientific Investigations Report 2005-5144 


\title{
U.S. Department of the Interior Gale A. Norton, Secretary
}

\author{
U.S. Geological Survey \\ P. Patrick Leahy, Acting Director
}

\section{U.S. Geological Survey, Reston, Virginia: 2005}

For sale by U.S. Geological Survey, Information Services

Box 25286, Denver Federal Center

Denver, CO 80225

For more information about the USGS and its products:

Telephone: 1-888-ASK-USGS

World Wide Web: http://www.usgs.gov/

Any use of trade, product, or firm names in this publication is for descriptive purposes only and does not imply endorsement by the U.S. Government.

Although this report is in the public domain, permission must be secured from the individual copyright owners to reproduce any copyrighted materials contained within this report.

Suggested citation:

Lee, C.J., Mau, D.P., and Rasmussen, T.J., 2005, Effects of nonpoint and selected point contaminant sources on stream-water quality and relation to land use in Johnson County, northeastern Kansas, October 2002 through June 2004: U.S. Geological Survey Scientific Investigations Report 2005-5144, 104 p.

Cover photograph:

High flow in Kill Creek at 95th Street near DeSoto, Kansas, August 31, 2003. 


\section{Contents}

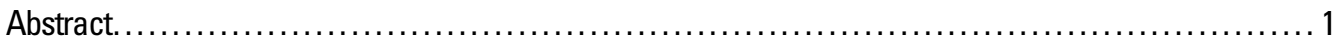

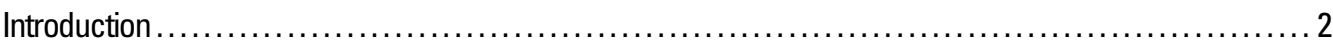

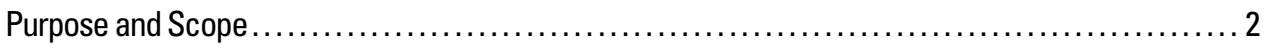

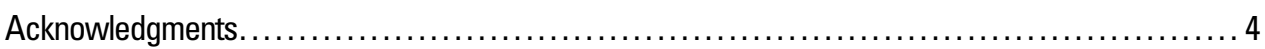

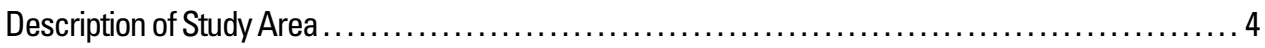

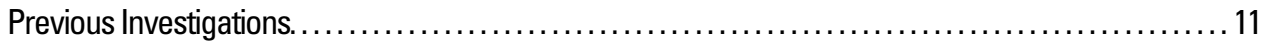

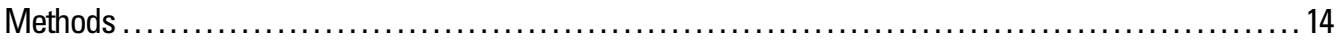

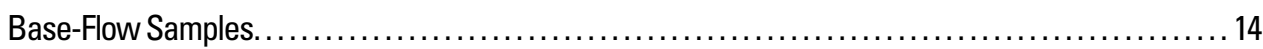

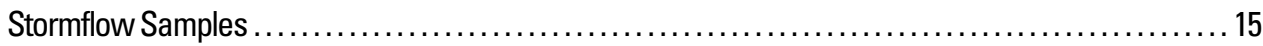

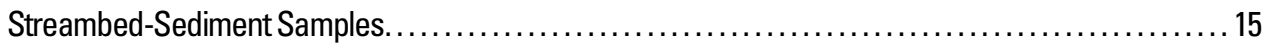

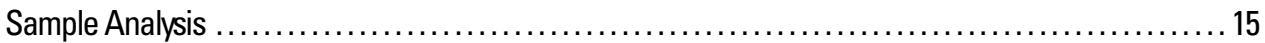

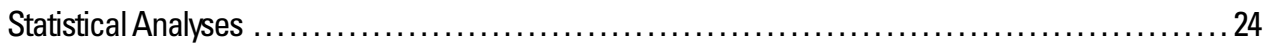

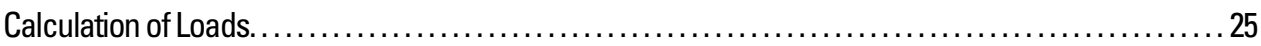

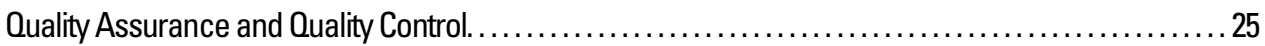

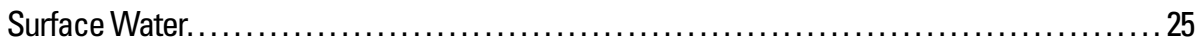

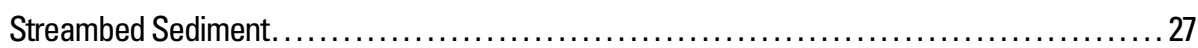

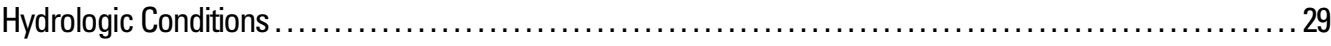

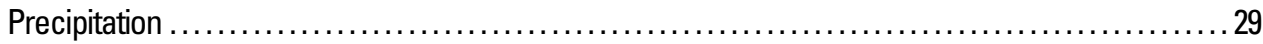

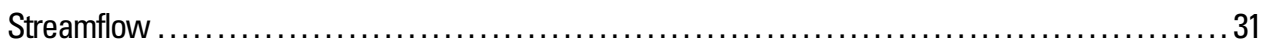

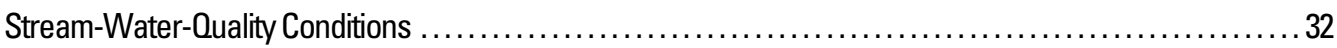

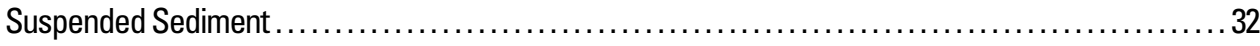

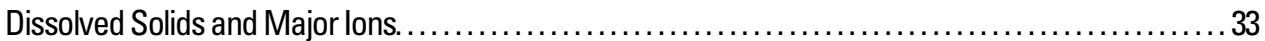

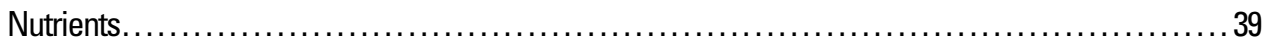

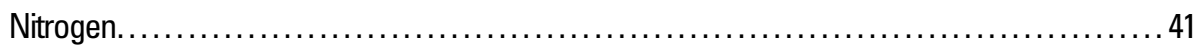

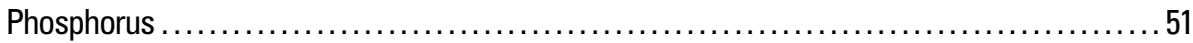

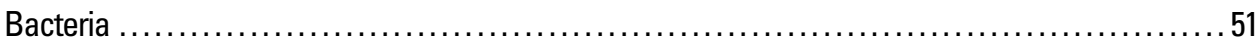

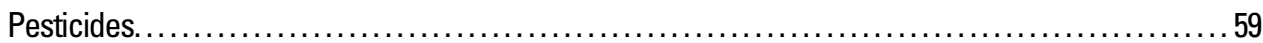

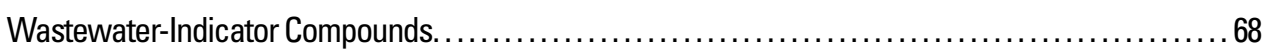

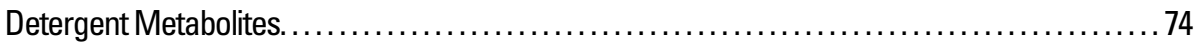

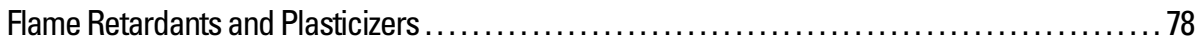

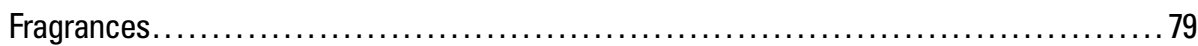

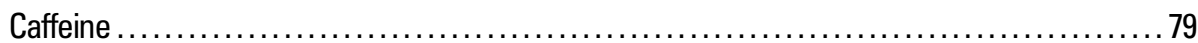

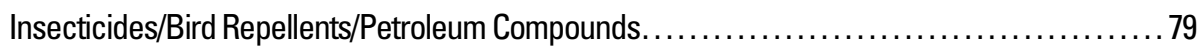

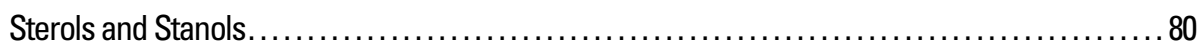

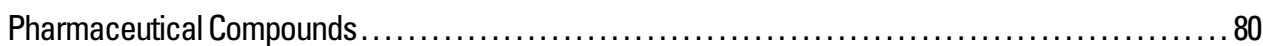

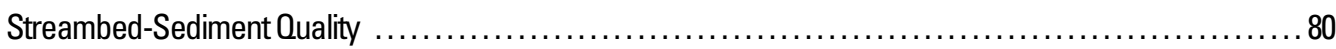

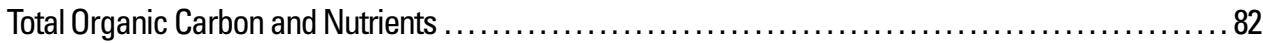

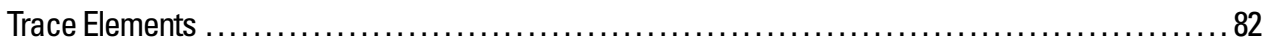

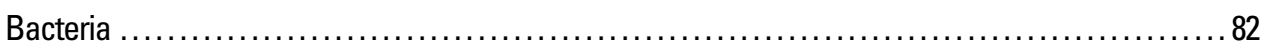

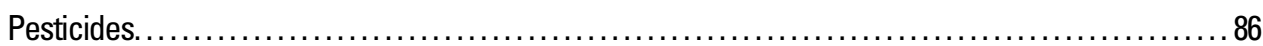

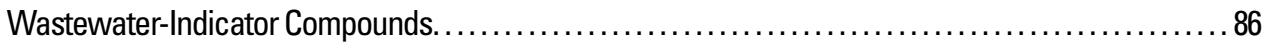

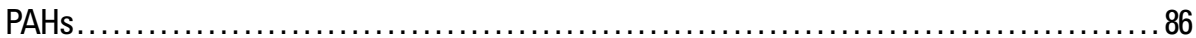




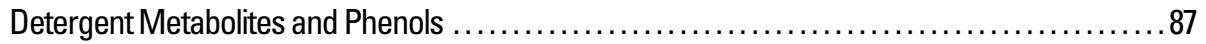

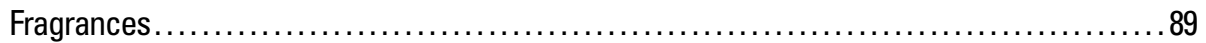

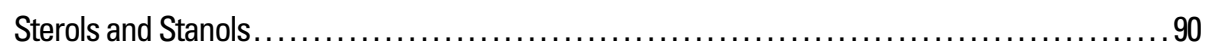

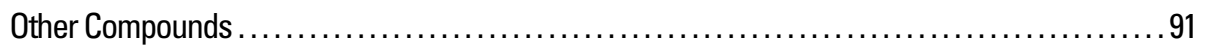

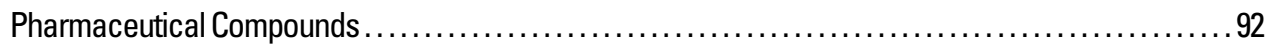

Summary of Stream-Water and Streambed-Sediment -Quality Data by Watershed . ................. 92

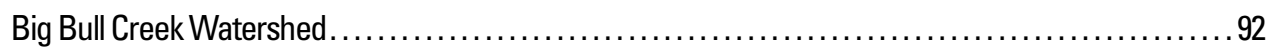

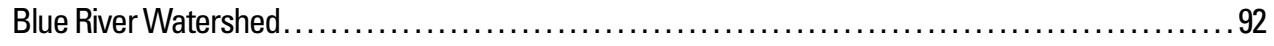

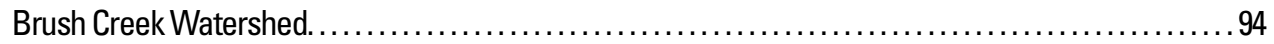

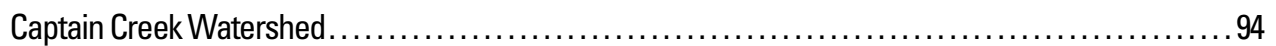

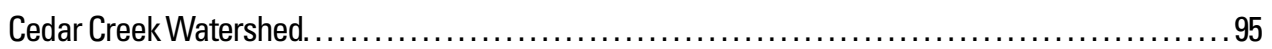

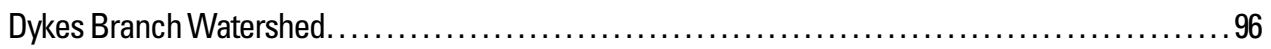

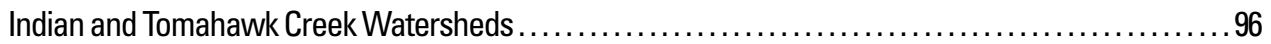

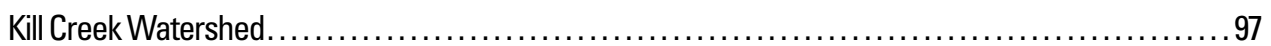

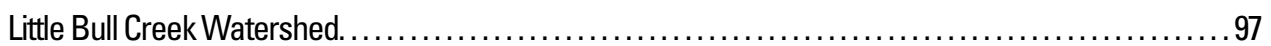

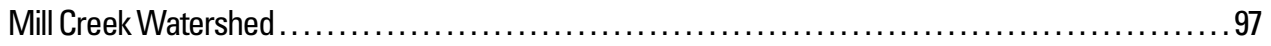

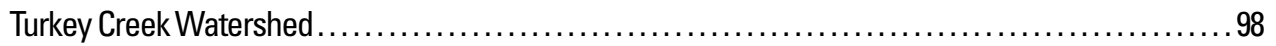

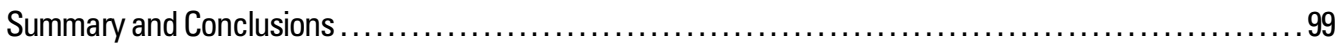

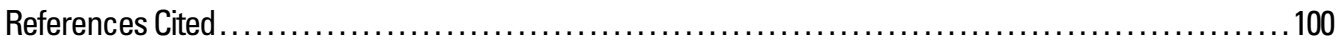

\section{Figures}

\section{1-3. Maps showing:}

1. Location of physiographic divisions, watershed boundaries, and sampling sites in Johnson County, northeastern Kansas, October 2002 through June $2004 . \ldots \ldots \ldots \ldots \ldots \ldots \ldots \ldots . \ldots \ldots$

2. Urban and nonurban land use in Johnson County, northeastern Kansas, 2003 . .............9

3. Agricultural and nonagricultural land use in Johnson County, northeastern Kansas, 2003 . . 12

4. Possible septic system locations in Johnson County, northeastern Kansas, 2003 .......... 13

5-36. Graphs showing:

5. Comparison of precipitation and streamflow at Mill Creek at Johnson Drive during streamwater sample collection, October 2002-June 2004.

6. Streamflow at base-flow sampling sites, November 4-7, 2002, and July 14-18, 2003 ....... 30

7. Estimated mean percentage of streamflow at the farthest downstream sampling site originating from wastewater-treatment facility discharges during base-flow sample collection, November 4-7, 2002, and July 14-18, 2003.....

8. Suspended-sediment concentrations in base- and stormflow samples collected at stormflow sampling sites, October 2002-June 2004.

9. Suspended-sediment concentrations and estimated loads in base- and stormflow samples, October 2002-June 2004.

10. Dissolved solids, magnesium, sodium, and chloride concentrations in base- and stormflow samples collected at stormflow sampling sites, October 2002-June 2004................ 39

11. Total nitrogen concentrations in base-flow samples, November 4-7, 2002, and

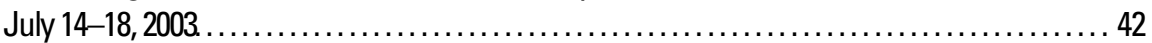

12. Total nitrogen concentrations in base- and stormflow samples collected at stormflow sampling

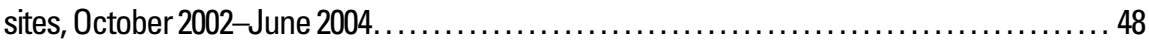

13. Total nitrogen concentrations and estimated loads in base- and stormflow samples, October 2002 through June 2004 
14. Percentage of dissolved nitrogen and dissolved phosphorus species in total nitrogen and phosphorus concentrations in samples collected at stormflow sampling sites under varying

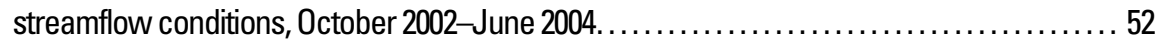

15. Total phosphorus concentrations in base-flow samples, November 4-7, 2002, and

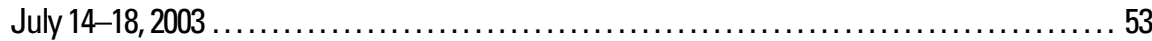

16. Total phosphorus concentrations in base-flow and stormflow samples collected at stormflow

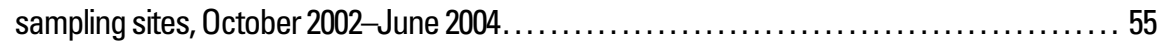

17. Total phosphorus concentrations and estimated loads in base- and stormflow samples,

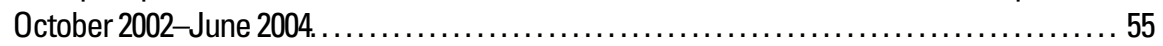

18. Fecal coliform bacteria densities in base-flow samples, November 4-7, 2002, and

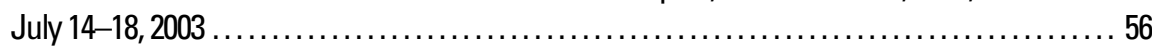

19. Escherichia coli densities in base-flow samples, November 4-7, 2002, and

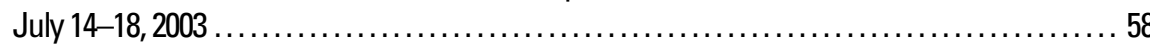

20. Indicator bacteria densities in base-flow and stormflow samples collected at stormflow

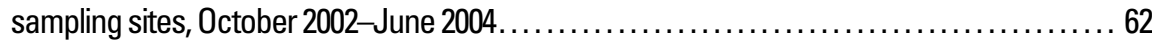

21. Escherichia colidensities and estimated loads in base- and stormflow samples, October 2002-June 2004................................................. 63

22. Atrazine concentrations in base-flow samples, November 4-7, 2002, and July 14-18, 2003 . . 67

23. Number of pesticide compound detections and total concentrations in stormflow samples,

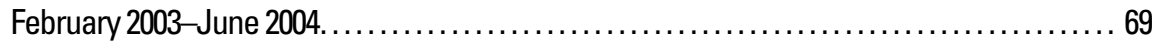

24. Number of organic wastewater-indicator compound detections and total concentrations in base-flow samples, November 4-7, 2002, and July 14-18, 2003.................... 72

25. Number of organic wastewater-indicator compound detections and total compound concentrations in stormflow samples, February 2003-April $2004 \ldots \ldots \ldots \ldots \ldots \ldots \ldots \ldots . \ldots 75$

26. Nonylphenol-diethoxylate concentrations in base-flow samples, November 4-7, 2002, and

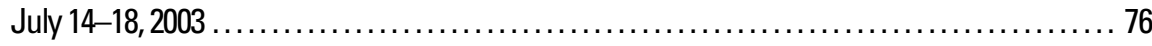

27. Nonylphenol-diethoxylate concentrations in stormflow samples, February 2003-April 2004 . 77

28. Nonylphenol-diethoxylate concentrations and estimated loads in base- and stormflow

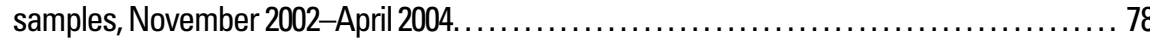

29 Number of pharmaceutical compound detections and total concentrations in selected baseflow samples, November 4-7, 2002, and July 14-18, 2003........................ 81

30. Total nitrogen and total phosphorus concentrations in streambed-sediment samples, March 31-April 3, 2003. . 83

31. Number of trace elements detected at concentrations greater than U.S. Environmental Protection Agency sediment-quality threshold effects level and probable effects level in Johnson County streambed-sediment samples, March 31-April 3, 2003............... 84

32. Indicator bacteria densities in streambed-sediment samples, March 31-April 3, 2003 ..... 85

33. Total chlordane concentrations in streambed-sediment samples, March 31-April 3, 2003 .. 87

34. Number of organic wastewater-indicator compound detections and total concentrations in streambed-sediment samples, March 31-April 3, 2003........................ 88

35. Nonylphenol concentrations in streambed-sediment samples, March 31-April 3, 2003 .... 90

36. Number of pharmaceutical compound detections and total concentrations in streambedsediment samples, March 31-April 3, 2003................................ 93

\section{Tables}

1. Watersheds containing stream segments with 303-(d) listings and (or) implemented total maximum daily loads, Johnson County, northeastern Kansas. 
2. Location and contributing drainage area of sampling sites in Johnson County, northeastern Kansas, October 2002-June 2004.

.5

3. Wastewater-treatment facilities sampled in Johnson County, northeastern Kansas, their designed

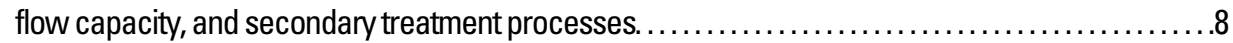

4. Estimates of urban and nonurban land-use percentages in selected watersheds of Johnson County, northeastern Kansas. 10

5. Stormflow sampling sites, contributing drainage areas, estimates of urban and nonurban land-use percentages, and estimated number of septic systems per square mile of drainage area in Johnson

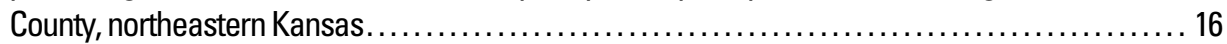

6. Constituents analyzed in stream-water and streambed-sediment samples collected from selected Johnson County streams, northeastern Kansas, October 2002-June 2004.

7. Mean relative percentage differences between environmental and replicate sample pairs with detections of dissolved solids, major ions, nutrients, trace elements, bacteria, and selected pesticide, wastewater, and pharmaceutical compounds in stream-water samples from Johnson County, northeastern Kansas, October 2002-June 2004 .

8. Mean relative percentage differences between environmental and replicate sample pairs with detections of nutrients, trace elements, bacteria, and pesticide, wastewater, and pharmaceutical compounds in streambed-sediment samples from Johnson County, northeastern Kansas, October 2002-June 2004.

9. Statistical summary of the results of streamflow measurements at Johnson County stormflow sampling sites, northeastern Kansas, October 2002-June 2004.

10. Statistical summary of the results of analysis of suspended sediment in samples collected from Johnson County stormflow sampling sites, northeastern Kansas, October 2002-June 2004.

11. Results of analysis of dissolved solids and major ions in water from base-flow sampling sites, Johnson County, northeastern Kansas, November 4-7, 2002, and July 14-18, 2003.

12. Statistical summary of the results of analysis of dissolved solids and major ions collected from Johnson County stormflow sampling sites, northeastern Kansas, October 2002-June 2004. 40

13. Results of analysis of nutrients in base-flow samples collected from selected Johnson County streams, northeastern Kansas, November 4-7, 2002, and July 14-18, 2003, level III ecoregion 40 criteria, and samples collected nationwide through the National Water-Quality Assessment Program, 1980-90.

14. Statistical summary of the results of analysis of nutrients collected from Johnson County stormflow sampling sites, northeastern Kansas, October 2002-June 2004, and samples collected nationwide through the National Water-Quality Assessment Program, 1980-90.

15. Percentage of base-flow samples collected up- and downstream from wastewater-treatment discharges that were equal to or exceeded Kansas Department of Health and Environment Escherichia coli and prior fecal coliform criteria for selected Johnson County streams, northeastern Kansas, October 2002-June 2004.

16. Median indicator bacteria densities in base-flow samples from watersheds with predominantly urban or nonurban land uses, Johnson County, northeastern Kansas, November 4-7, 2002, and July 14-18, 2003.

17. Results of regression analysis for bacteria densities with streamflow and suspended-sediment concentrations for selected Johnson County streams, northeastern Kansas, October 2002June 2004

18. Median and maximum concentrations of pesticide compounds detected at greater than laboratory reporting levels in base-flow and stormflow samples collected from selected Johnson County streams, northeastern Kansas, October 2002-June 2004, and samples collected nationwide for the U.S. Geological Survey's National Water-Quality Assessment cycle-I studies, 1992-2001 . . . . . . . 65

19. Organic wastewater-indicator compounds analyzed in stream-water samples from selected streams in Johnson County, northeastern Kansas, October 2002-June 2004 . 
20. Median and maximum concentrations of organic wastewater-indicator compounds detected at concentrations larger than laboratory reporting levels in base-flow samples from selected Johnson County streams, northeastern Kansas, November 4-7, 2002, and July 14-18, 2003, and samples collected nationwide by the U.S. Geological Survey, 1999-2000, and in Minnesota, 2000-02 . ......73

21. Concentrations of polycyclic aromatic hydrocarbons and metabolites in streambed-sediment samples collected from selected Johnson County streams, northeastern Kansas, March 31-

April 3, 2003.

22. Concentrations of fragrance compounds in streambed-sediment samples collected from selected Johnson County streams, northeastern Kansas, March 31-April 3, 2003 


\section{Conversion Factors, Abbreviations, and Datum}

\begin{tabular}{lll}
\hline Multiply & By & To obtain \\
\hline cubic foot per second $\left(\mathrm{ft}^{3} / \mathrm{s}\right)$ & 0.02832 & cubic meter per second $\left(\mathrm{m}^{3} / \mathrm{s}\right)$ \\
cubic foot per second $\left(\mathrm{ft}^{3} / \mathrm{s}\right)$ & 0.6463 & million gallons per day $(\mathrm{Mgal} / \mathrm{d})$ \\
gram $(\mathrm{g})$ & 0.035274 & ounce $(\mathrm{oz})$ \\
inch $(\mathrm{in})$. & 2.54 & centimeter $(\mathrm{cm})$ \\
kilogram $(\mathrm{kg})$ & 2.205 & pound $(\mathrm{lb})$ \\
kilogram per day $(\mathrm{kg} / \mathrm{d})$ & 2.205 & pound per day $(\mathrm{lb} / \mathrm{d})$ \\
liter $(\mathrm{L})$ & 33.82 & ounce, fluid $(\mathrm{oz})$ \\
microgram per kilogram $(\mu \mathrm{g} / \mathrm{kg})$ & 0.00000016 & ounce per pound $(\mathrm{oz} / \mathrm{lb})$ \\
microgram per liter $(\mu \mathrm{g} / \mathrm{L})$ & 1.0 & part per billion $(\mathrm{ppb})$ \\
micrometer $(\mu \mathrm{m})$ & 0.00003937 & inch $($ in. $)$ \\
mile $(\mathrm{mi})$ & 1.609 & kilometer $(\mathrm{km})$ \\
milligram $(\mathrm{mg})$ & 0.0000353 & ounce $(\mathrm{oz})$ \\
milligram per gram $(\mathrm{mg} / \mathrm{g})$ & 0.016 & ounce per pound $(\mathrm{oz} / \mathrm{lb})$ \\
milligram per kilogram $(\mathrm{mg} / \mathrm{kg})$ & 0.000016 & ounce per pound $(\mathrm{oz} / \mathrm{lb})$ \\
milligram per liter $(\mathrm{mg} / \mathrm{L})$ & 1.0 & part per million $(\mathrm{ppm})$ \\
milliliter $(\mathrm{mL})$ & 0.0338 & ounce, fluid $(\mathrm{oz})$ \\
million gallons per day $(\mathrm{Mgal} / \mathrm{d})$ & 0.04381 & cubic meter per second $\left(\mathrm{m}^{3} / \mathrm{s}\right)$ \\
ounce $(\mathrm{oz}$, fluid) & 0.02957 & liter $(\mathrm{L})$ \\
pound $(\mathrm{lb})$ & 0.4536 & kilogram $(\mathrm{kg})$ \\
pound per square inch $\left(\mathrm{lb} / \mathrm{in}^{2}\right)$ & 6.895 & kilopascal $(\mathrm{kPa})$ \\
square mile $\left(\mathrm{mi}{ }^{2}\right)$ & 2.590 & square kilometer $\left(\mathrm{km}{ }^{2}\right)$ \\
\hline
\end{tabular}

Temperature in degrees Celsius $\left({ }^{\circ} \mathrm{C}\right)$ may be converted to degrees Fahrenheit $\left({ }^{\circ} \mathrm{F}\right)$ as follows:

${ }^{\circ} \mathrm{F}=\left(1.8 \mathrm{x}^{\circ} \mathrm{C}\right)+32$.

Temperature in degrees Fahrenheit $\left({ }^{\circ} \mathrm{F}\right)$ may be converted to degrees Celsius $\left({ }^{\circ} \mathrm{C}\right)$ as follows:

${ }^{\circ} \mathrm{C}=\left({ }^{\circ} \mathrm{F}-32\right) / 1.8$.

Horizontal coordinate information is referenced to the North American Datum of 1983 (NAD 83).

Specific conductance is given in microsiemens per centimeter at 25 degrees Celsius $(\mu \mathrm{S} / \mathrm{cm}$ at $\left.25^{\circ} \mathrm{C}\right)$.

Concentrations of chemical constituents in water are given either in milligrams per liter (mg/L) or micrograms per liter $(\mu \mathrm{g} / \mathrm{L})$.

Concentrations of chemical constituents in streambed sediment are given in milligrams per kilogram $(\mathrm{mg} / \mathrm{kg})$ or micrograms per kilogram $(\mu \mathrm{g} / \mathrm{kg})$.

Bacteria densities are given in colonies per 100 milliliters $(\mathrm{col} / 100 \mathrm{~mL}$ ) of water and colonies per gram dry weight (col/GDW). 


\title{
Effects of Nonpoint and Selected Point Contaminant Sources on Stream-Water Quality and Relation to Land Use in Johnson County, Northeastern Kansas, October 2002 Through June 2004
}

\author{
By Casey J. Lee, David P. Mau, and Teresa J. Rasmussen
}

\section{Abstract}

Water and sediment samples were collected by the U.S. Geological Survey in 12 watersheds in Johnson County, northeastern Kansas, to determine the effects of nonpoint and selected point contaminant sources on stream-water quality and their relation to varying land use. The streams studied were located in urban areas of the county (Brush, Dykes Branch, Indian, Tomahawk, and Turkey Creeks), developing areas of the county (Blue River and Mill Creek), and in more rural areas of the county (Big Bull, Captain, Cedar, Kill, and Little Bull Creeks). Two base-flow synoptic surveys (73 total samples) were conducted in 11 watersheds, a minimum of three stormflow samples were collected in each of six watersheds, and 15 streambed-sediment sites were sampled in nine watersheds from October 2002 through June 2004.

Discharge from seven wastewater treatment facilities (WWTFs) were sampled during base-flow synoptic surveys. Discharge from these facilities comprised greater than 50 percent of streamflow at the farthest downstream sampling site in six of the seven watersheds during base-flow conditions. Nutrients, organic wastewater-indicator compounds, and prescription and nonprescription pharmaceutical compounds generally were found in the largest concentrations during base-flow conditions at sites at, or immediately downstream from, pointsource discharges from WWTFs. Downstream from WWTF discharges streamflow conditions were generally stable, whereas nutrient and wastewater-indicator compound concentrations decreased in samples from sites farther downstream.

During base-flow conditions, sites upstream from WWTF discharges had significantly larger fecal coliform and Escherichia coli densities than downstream sites. Stormflow samples had the largest suspended-sediment concentrations and indicator bacteria densities. Other than in samples from sites in proximity to WWTF discharges, stormflow samples generally had the largest nutrient concentrations in Johnson County streams.

Discharge from WWTFs with trickling-filter secondary treatment processes had the largest concentrations of many potential contaminants during base-flow conditions. Samples from two of three trickling-filter WWTFs exceeded Kansas
Department of Health and Environment $\mathrm{pH}$ - and temperaturedependent chronic aquatic-life criteria for ammonia when earlylife stages of fish are present. Discharge from trickling-filter facilities generally had the most detections and largest concentrations of many organic wastewater-indicator compounds in Johnson County stream-water samples. Caffeine (stimulant), nonylphenol-diethoxylate (detergent surfactant), and tris(2butoxyethyl) phosphate (floor polish, flame retardant, and plasticizer) were found at concentrations larger than maximum concentrations in comparable studies.

Land use and seasonality affected the occurrence and magnitude of many potential water-quality contaminants originating from nonpoint sources. Base-flow samples from urban sites located upstream from WWTF discharges had larger indicator bacteria densities and wastewater-indicator compound concentrations than did base-flow samples from sites in nonurban areas. Dissolved-solids concentrations were the largest in winter stormflow samples from urban sites and likely were due to runoff from road-salt application. One sample from an urban watershed had a chloride concentration of 1,000 milligrams per liter, which exceeded the Kansas Department of Health and Environment's acute aquatic-life use criterion (860 milligrams per liter) likely due to effects from road-salt application. Pesticide concentrations were the largest in spring stormflow samples collected in nonurban watersheds. Although most wastewater-indicator compounds were found at the largest concentrations in samples from WWTF discharges, the compounds 9-10, anthraquinone (bird repellent), caffeine (stimulant), carbazole (component of coal tar, petroleum products), nonylphenol-diethoxylate (detergent surfactant), and tris(2butoxyethyl) phosphate (flame retardant and plasticizer) also were detected in larger than microgram-per-liter concentrations in stormflow samples primarily from sites downstream from urban watersheds.

The effects of point contaminant sources and varying land use also were observable in streambed-sediment samples. Nutrients, silver, and many wastewater-indicator and prescription and nonprescription pharmaceutical compounds had the largest concentrations in streambed-sediment samples collected immediately downstream from WWTF discharges. Trace elements, total chlordane, total dichloro-diphenyl-trichloroethane 


\section{Effects of Contaminant Sources on Stream-Water Quality and Relation to Land Use in Johnson County, Northeast Kansas}

(DDT), polyaromatic hydrocarbons (PAHs), and selected wastewater-indicator compounds were not as linked to WWTF discharges and had the largest concentrations in streambedsediment samples from watersheds with primarily urban land uses. Information provided in this report can be used by Johnson County officials to help define current (2005) waterquality conditions and to identify source areas of water-quality contamination.

\section{Introduction}

Johnson County is the fastest growing and most populous county in Kansas. The population has increased by 238 percent since 1960 and by 37 percent from 1990 to 2003. The approximate population was 486,500 residents in 2003 (U.S. Census Bureau, 2004). Population increases lead to land-use changes that can affect the water quality of local streams. Point-source discharges, such as wastewater-treatment facility (WWTF) discharges, as well as nonpoint-source discharges, such as stormwater runoff from urban and agricultural watersheds, sanitary sewer overflows, and contamination from failing septic systems can degrade stream-water quality.

Contaminant sources in Johnson County streams result in many of the stream segments not meeting designated uses. The Kansas Department of Health and Environment (KDHE) has listed the designated uses and impairments of stream segments on the biennial 303-(d) list and has developed or plans to develop total maximum daily loads (TMDLs) to address impairments. Five watersheds (Blue River, Cedar Creek, Indian Creek, Kill Creek, and Mill Creek) within Johnson County (fig. 1) have stream segments that have been listed on the 303-(d) list or have had TMDLs developed for impaired water quality by KDHE (table 1) (Kansas Department of Health and Environment, 2004b). TMDLs are quantitative standards and include strategies designed to achieve water-quality standards. The standards constitute goals to fully support the designated uses of streams, lakes, and wetlands. KDHE has conducted sampling within these five watersheds as part of the statewide monitoring program since 1988 (Kansas Department of Health and Environment, 2004b).

Some municipalities in Johnson County are subject to the U.S. Environmental Protection Agency (USEPA) National Pollutant Discharge and Elimination System (NPDES) Phase II Stormwater Program regulations designed to reduce the effects of stormwater runoff on stream-water quality. NPDES Phase II regulations require permittees to establish best management practices to reduce stream-water impacts from stormwater runoff (U.S. Environmental Protection Agency, 2005a).

Stream-water contamination often is identified through the quantification of contaminants such as suspended sediment, nutrients, metals, bacteria, and pesticides. These contaminants can pose human-health risks for drinking and recreational water uses; inhibit the growth, reproduction, and diversity of aquatic organisms; and degrade aesthetic stream qualities. In addition, many of these chemical constituents often occur in larger concentrations in streambed sediment than in the overlying water column (Van Donsel and Geldreich, 1971; Horowitz, 1991). Contaminated sediment can be toxic to benthic organisms, and the contaminants can bioaccumulate in fish, insects, and mammals (U.S. Environmental Protection Agency, 2000a).

New analytical techniques and sampling methodologies recently have become available that allow for the detection of organic wastewater-indicator compounds associated with household chemicals (herein identified as wastewater compounds) and over-the-counter and prescription pharmaceuticals (herein identified as pharmaceutical compounds) that typically are discharged to the environment by WWTFs (Kolpin and others, 2002). The effects of many of these wastewater and pharmaceutical compounds are poorly understood, although some are known or suspected endocrine disrupters (Daughton and Ternes, 1999). Detection of these compounds in stream water can indicate that a human-waste source is contributing to the stream (Wilkison and others, 2002). Traditional measures of water-quality degradation combined with new methodologies for measuring contaminant sources can provide a more comprehensive assessment of stream-water quality in Johnson County.

In 2002, the U.S. Geological Survey (USGS), in cooperation with the Johnson County Stormwater Management Program, began an investigation in Johnson County to characterize stream-water-quality conditions. Initial study efforts were designed to describe the effects of nonpoint and selected point contaminant sources on stream-water quality and their relation to land use. Data collected through 2006 are geared toward estimating water-quality constituent loads in watersheds with different land-use characteristics.

\section{Purpose and Scope}

The purpose of this report is to describe the effects of nonpoint and selected point contaminant sources on water-quality conditions in Johnson County streams and their relation to land use using analytical results from stream-water and streambedsediment samples. Data were evaluated to determine the distribution of potential water-quality contaminants relative to contaminant sources and varying land-use characteristics. The report describes the results of two synoptic stream-water sample collections during base-flow conditions (73 samples) in 11 watersheds; collection of at least three stream-water samples from each of six watersheds during stormflow conditions, and collection of 15 streambed-sediment samples in nine watersheds. Data were summarized by constituent and by watershed to provide both a county-wide and an individual watershed perspective on data collected from stream-water and streambedsediment samples. All base-flow water samples were analyzed for suspended sediment, dissolved solids, major ions, nutrients, bacteria, pesticides, and wastewater compounds. In addition, water samples collected from selected sites during base-flow conditions were analyzed for selected over-the-counter and prescription pharmaceutical compounds. Stormflow samples were analyzed for suspended sediment, dissolved solids, major ions, nutrients, bacteria, and pesticide compounds. Selected 


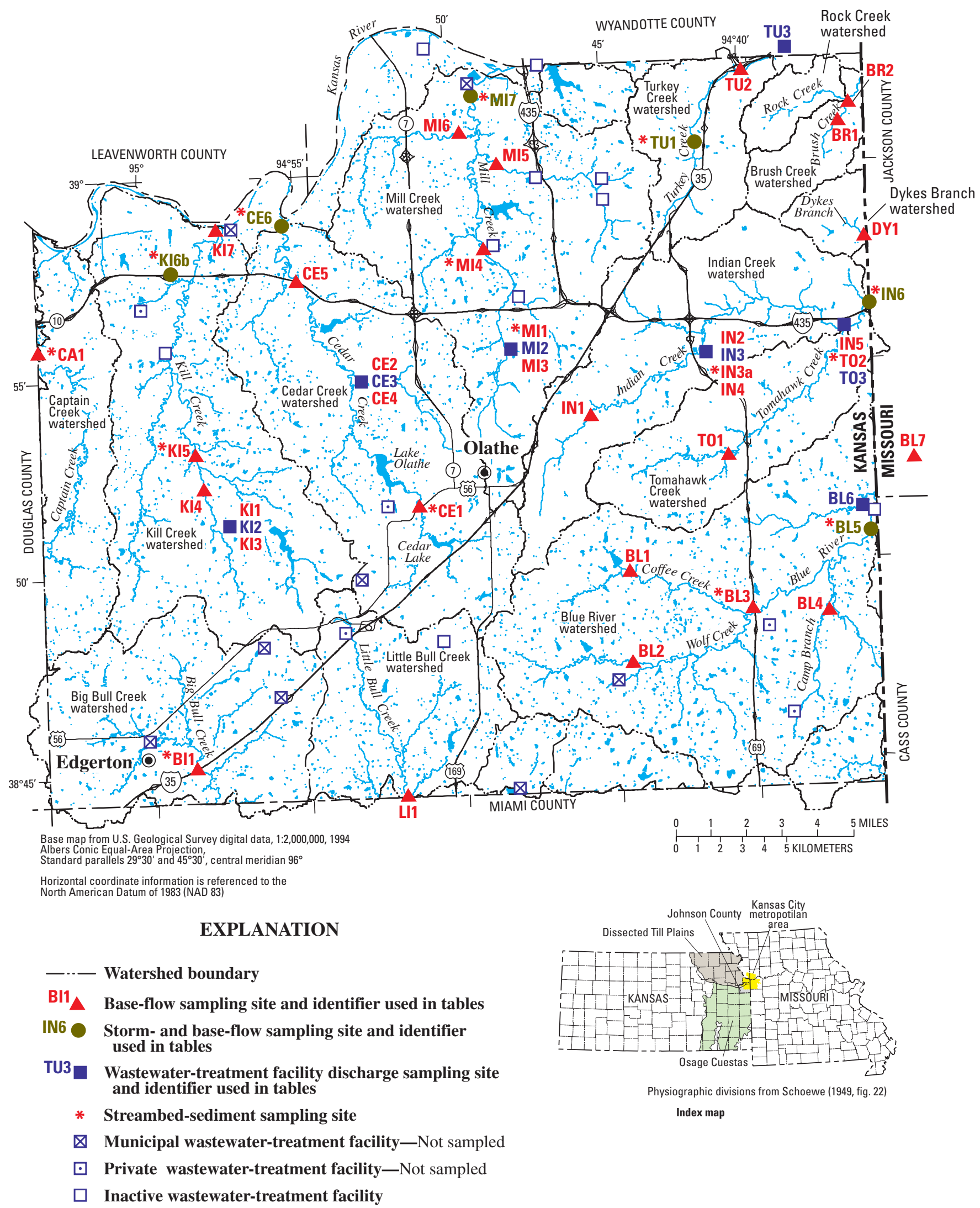

Figure 1. Location of physiographic divisions, watershed boundaries, and sampling sites in Johnson County, northeastern Kansas, October 2002 through June 2004. 
Table 1. Watersheds containing stream segments with 303-(d) listings and (or) implemented total maximum daily loads (TMDLs), Johnson County, northeastern Kansas.

[Data from Kansas Department of Health and Environment, 2004b; X, 303-(d) listing and total maximum daily load implemented; --, no total maximum daily load]

\begin{tabular}{|c|c|c|c|c|c|c|c|c|c|}
\hline \multirow[b]{2}{*}{$\begin{array}{l}\text { Watershed } \\
\text { (fig. 1) }\end{array}$} & \multicolumn{9}{|c|}{ 303-(d) listing and (or) implemented TMDLs } \\
\hline & $\begin{array}{c}\text { Dissolved } \\
\text { oxygen }\end{array}$ & $\begin{array}{c}\text { Sediment/ } \\
\text { biological } \\
\text { impact }^{1}\end{array}$ & Chloride & $\begin{array}{l}\text { Nitrates/ } \\
\text { nitrites }\end{array}$ & $\begin{array}{c}\text { Nutrients/ } \\
\text { biological } \\
\text { oxygen } \\
\text { demand }^{1}\end{array}$ & Copper & Zinc & $\begin{array}{c}\text { Fecal } \\
\text { coliform } \\
\text { bacteria }\end{array}$ & Chlordane $^{2}$ \\
\hline Blue River & $\mathrm{X}$ & -- & -- & -- & $\mathrm{X}$ & -- & -- & $\mathrm{X}$ & $\mathrm{X}$ \\
\hline Cedar Creek & -- & -- & -- & $\mathrm{X}$ & -- & -- & -- & $\mathrm{X}$ & -- \\
\hline Kill Creek & -- & -- & -- & -- & -- & $X$ & -- & $\mathrm{X}$ & $\mathrm{X}$ \\
\hline Mill Creek & -- & $\mathrm{X}$ & $\mathrm{X}$ & -- & $\mathrm{X}$ & -- & $\mathrm{X}$ & $\mathrm{X}$ & -- \\
\hline
\end{tabular}

${ }^{1}$ Impairment identified by biological monitoring.

${ }^{2}$ Impairment identified by fish tissue analysis.

stormflow samples also were analyzed for wastewater compounds. Streambed-sediment samples were analyzed for total organic carbon, total carbon, nutrients, trace elements, bacteria, pesticides, and wastewater and pharmaceutical compounds.

This report identifies potential contaminants that are most prevalent in Johnson County streams and the source areas contributing these contaminants. The identification of areas with large contaminant contributions will allow for more effective implementation of best management practices and aid in determining their success. These results will support Federal, State, and local watershed strategies designed to improve water quality and help to identify the sources and mode of transport of wastewater and pharmaceutical compounds in the environment.

\section{Acknowledgments}

The authors are grateful to Tony Holt and Pedro Calderon at the Johnson County Laboratory (Johnson County, Kansas) for helping to coordinate laboratory and onsite sampling efforts during all aspects of the study. Appreciation is also expressed to Shannon Porter of the Johnson County Automated Information Mapping System (AIMS) and Kevin Skridulis of the Johnson County Appraiser's Office for their assistance in obtaining and working with geographic information system data and to Eileen Hack of the Johnson County Wastewater and Public Works Department for technical assistance.

\section{Description of Study Area}

Johnson County, Kansas, consists of $477 \mathrm{mi}^{2}$ of surface area located in the western part of the Kansas City metropolitan area (U.S. Census Bureau, 2004). The county contains all or part of 17 watersheds, 13 of which are within the sampling-site network described in this report. These watersheds are the Big Bull Creek, Blue River, Brush Creek, Captain Creek, Cedar
Creek, Dykes Branch, Indian Creek, Kill Creek, Little Bull Creek, Mill Creek, Rock Creek (upstream from Brush Creek site BR2), Tomahawk Creek, and Turkey Creek watersheds (fig. 1, table 2). Designated uses for streams within the county include aquatic-life support, contact recreation, drinking-water supply, food procurement, ground-water recharge, irrigation, industrial use, and livestock watering.

Fifteen municipal WWTFs (seven of which were sampled in this study) and five private WWTFs are located within Johnson County watersheds (fig. 1). Private WWTFs are not significant sources of streamflow to Johnson County streams (E. Hack, Johnson County Wastewater and Public Works, written commun, 2005). In addition, there are 10 inactive WWTFs that previously discharged to Johnson County streams (fig. 1). Table 3 lists WWTFs sampled in Johnson County, their designed flow capacity, and secondary treatment processes (E. Hack, Johnson County Wastewater and Public Works, written commun., 2003). Johnson County WWTFs sampled include facilities with either activated-sludge or trickling-filter secondary treatment processes. Activated-sludge treatment facilities use microorganisms in a mixed sludge in combination with aeration to degrade organic material in wastewater. Trickling-filter facilities force wastewater to flow over a medium enriched with microorganisms that degrade organic material in the wastewater stream. Activated-sludge treatment facilities have been shown to be more efficient than tricklingfilter facilities at removing many wastewater compounds (Phillips and others, 2004). Wastewater and pharmaceutical compounds currently (2005) do not have NPDES regulations, and WWTFs in Johnson County are not specifically designed to remove these compounds.

Johnson County lies within the Attenuated Drift Border of the Dissected Till Plains physiographic section and partly within the Osage Cuestas of the Osage Plains physiographic section (fig. 1) (Schoewe, 1949). The county mostly consists of gently rolling uplands with hilly areas along the streams. The 
Table 2. Location and contributing drainage area of sampling sites in Johnson County, northeastern Kansas, 0 ctober $2002-J u n e ~ 2004$.

$\left[\mathrm{mi}^{2}\right.$, square miles; --, not applicable]

\begin{tabular}{|c|c|c|c|c|c|c|c|}
\hline $\begin{array}{l}\text { Sampling } \\
\text { site } \\
\text { identifier } \\
\text { (fig. 1) }\end{array}$ & $\begin{array}{c}\text { U.S. Geological } \\
\text { Survey identification } \\
\text { number }\end{array}$ & Site name & $\begin{array}{c}\text { Contributing } \\
\text { drainage area in } \\
\text { Johnson County } \\
\left(\mathrm{mi}^{2}\right)\end{array}$ & $\begin{array}{c}\text { Latitude } \\
\text { (degrees, minutes, } \\
\text { seconds) }\end{array}$ & $\begin{array}{c}\text { Longitude } \\
\text { (degrees, minutes, } \\
\text { seconds) }\end{array}$ & Sample type ${ }^{1}$ & $\begin{array}{c}\text { Contact } \\
\text { recreation } \\
\text { classification }{ }^{2}\end{array}$ \\
\hline \multicolumn{8}{|c|}{ Big Bull Creek watershed } \\
\hline BI1 & 06914950 & Big Bull Creek near Edgerton & 26.5 & $38^{\circ} 45^{\prime} 12^{\prime \prime}$ & $94^{\circ} 58^{\prime} 38^{\prime \prime}$ & $1,2, \mathrm{~S}$ & -- \\
\hline \multicolumn{8}{|c|}{ Blue River watershed } \\
\hline BL1 & 384951094443200 & Coffee Creek at Pflumm Road & 8.3 & $38^{\circ} 49^{\prime} 51^{\prime \prime}$ & $94^{\circ} 44^{\prime} 32^{\prime \prime}$ & -- & -- \\
\hline BL2 & 384732094443200 & Wolf Creek at Pflumm Road & 9.7 & $38^{\circ} 47^{\prime} 32^{\prime \prime}$ & $94^{\circ} 44^{\prime} 32^{\prime \prime}$ & -- & -- \\
\hline BL3 & 06893080 & Blue River near Stanley & 46.5 & $38^{\circ} 48^{\prime} 45^{\prime \prime}$ & $94^{\circ} 40^{\prime} 33^{\prime \prime}$ & $2, \mathrm{~S}$ & $\mathrm{C}$ \\
\hline BL4 & 384840094381100 & Camp Branch at 175 th Street & 6.7 & $38^{\circ} 48^{\prime} 40^{\prime \prime}$ & $94^{\circ} 38^{\prime} 11^{\prime \prime}$ & 2 & $\mathrm{~b}$ \\
\hline BL5 & 06893100 & $\begin{array}{l}\text { Blue River at Kenneth Road, Overland } \\
\text { Park }\end{array}$ & 65.7 & $38^{\circ} 50^{\prime} 32^{\prime \prime}$ & $94^{\circ} 36^{\prime} 44^{\prime \prime}$ & $2, \mathrm{~S}, \mathrm{R}$ & $\mathrm{C}$ \\
\hline BL6 & 385114094365800 & $\begin{array}{l}\text { Discharge from Blue River Main } \\
\text { Number } 1 \text { wastewater-treatment facility }\end{array}$ & 0 & $38^{\circ} 51^{\prime} 14^{\prime \prime}$ & $94^{\circ} 36^{\prime} 59^{\prime \prime}$ & $1,2, \mathrm{~W}$ & $\mathrm{C}$ \\
\hline BL7 & 385226094351500 & Blue River at Holmes Road, Missouri & 75.5 & $38^{\circ} 52^{\prime} 26^{\prime \prime}$ & $94^{\circ} 35^{\prime} 16^{\prime \prime}$ & 1,2 & $\mathrm{C}$ \\
\hline \multicolumn{8}{|c|}{ Brush Creek watershed } \\
\hline BR1 & 390056094371600 & Brush Creek at 63rd Street & 5.5 & $39^{\circ} 00^{\prime} 56^{\prime \prime}$ & $94^{\circ} 37^{\prime} 17^{\prime \prime}$ & 1,2 & -- \\
\hline BR2 & 390127094365800 & Brush Creek at Belinder Street & 11.1 & $39^{\circ} 01^{\prime} 27^{\prime}$ & $94^{\circ} 36^{\prime} 58^{\prime \prime}$ & 1,2 & -- \\
\hline \multicolumn{8}{|c|}{ Captain Creek watershed } \\
\hline CA1 & 385540095032800 & Captain Creek near 119th Street & 16.0 & $38^{\circ} 55^{\prime} 40^{\prime \prime}$ & $95^{\circ} 03^{\prime} 28^{\prime \prime}$ & $S$ & $\mathrm{C}$ \\
\hline \multicolumn{8}{|c|}{ Cedar Creek watershed } \\
\hline CE1 & 06892440 & Cedar Creek at Highway 56 at Olathe & 13.2 & $38^{\circ} 51^{\prime} 33^{\prime \prime}$ & $94^{\circ} 51^{\prime} 14^{\prime \prime}$ & $1,2, \mathrm{~S}$ & $\mathrm{C}$ \\
\hline CE2 & 385437094531800 & $\begin{array}{l}\text { Cedar Creek near Cedar Creek } \\
\text { wastewater-treatment facility }\end{array}$ & 25.4 & $38^{\circ} 54^{\prime} 38^{\prime \prime}$ & $94^{\circ} 53^{\prime} 18^{\prime \prime}$ & 2 & $\mathrm{C}$ \\
\hline CE3 & 385438094531800 & $\begin{array}{l}\text { Discharge from Cedar Creek } \\
\text { wastewater-treatment facility }\end{array}$ & 0 & $38^{\circ} 54^{\prime} 38^{\prime \prime}$ & $94^{\circ} 44^{\prime} 32^{\prime \prime}$ & $1,2, \mathrm{~W}$ & $\mathrm{C}$ \\
\hline CE4 & 385445094531900 & Cedar Creek at 119th Street & 25.4 & $38^{\circ} 54^{\prime} 46^{\prime \prime}$ & $94^{\circ} 53^{\prime} 19^{\prime \prime}$ & 2 & $\mathrm{C}$ \\
\hline CE5 & 385722094545500 & Cedar Creek at Highway 10 & 47.2 & $38^{\circ} 57^{\prime} 22^{\prime \prime}$ & $94^{\circ} 54^{\prime} 56^{\prime \prime}$ & 1,2 & $\mathrm{C}$ \\
\hline CE6 & 06892495 & Cedar Creek near DeSoto & 58.5 & $38^{\circ} 58^{\prime} 41^{\prime \prime}$ & $94^{\circ} 55^{\prime} 20^{\prime \prime}$ & $1,2, \mathrm{R}, \mathrm{S}$ & $\mathrm{C}$ \\
\hline \multicolumn{8}{|c|}{ Dykes Branch watershed } \\
\hline DY1 & 385804094363600 & Dykes Branch Creek at 89th Street & 3.4 & $38^{\circ} 58^{\prime} 04^{\prime \prime}$ & $94^{\circ} 36^{\prime} 36^{\prime \prime}$ & 1,2 & -- \\
\hline
\end{tabular}


Table 2. Location and contributing drainage area of sampling sites in Johnson County, northeastern Kansas, 0ctober 2002-June 2004.-Continued

$\left[\mathrm{mi}^{2}\right.$, square miles; --, not applicable]

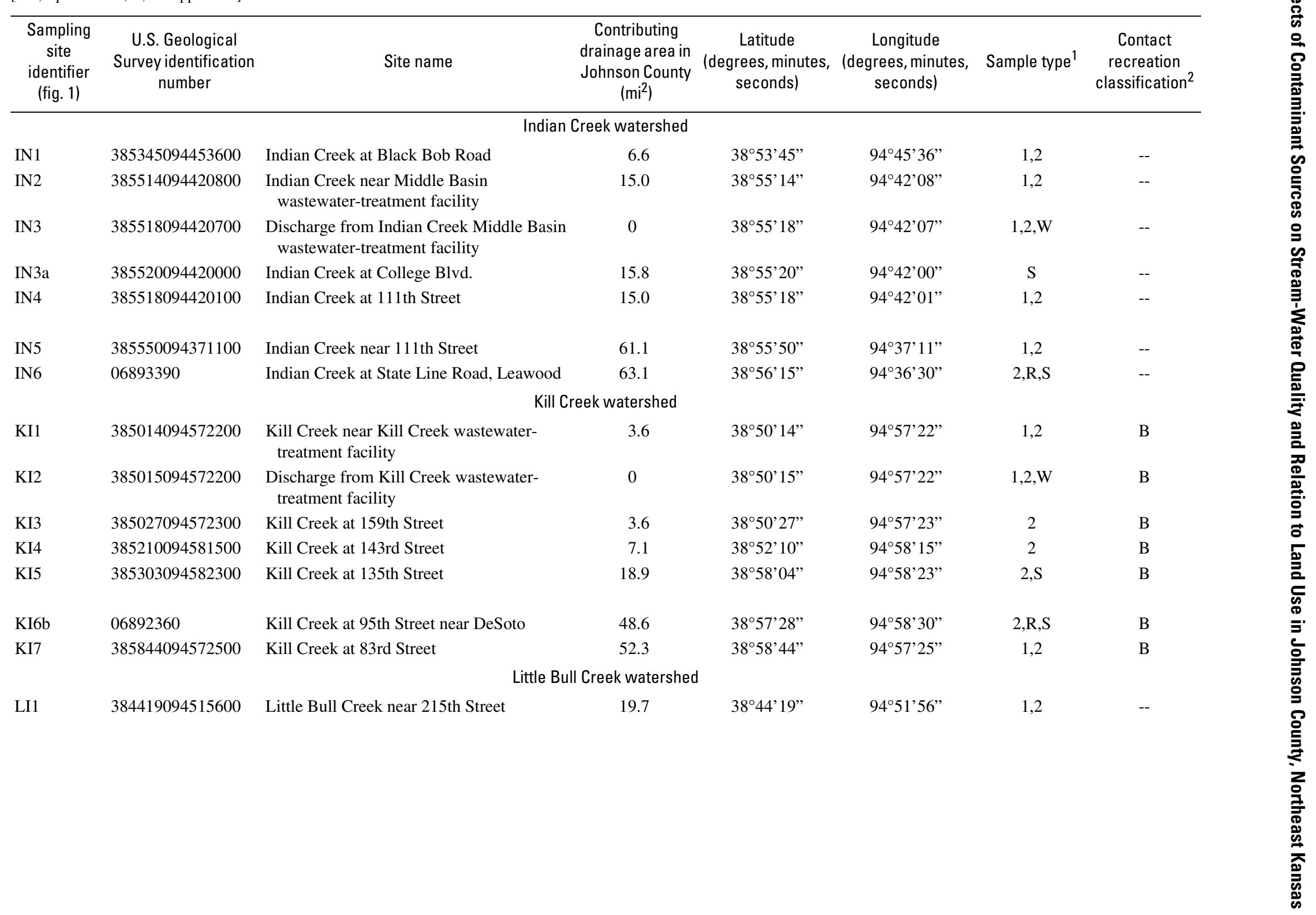


Table 2. Location and contributing drainage area of sampling sites in Johnson County, northeastern Kansas, October 2002-June 2004.-Continued

$\left[\mathrm{mi}^{2}\right.$, square miles; --, not applicable]

\begin{tabular}{|c|c|c|c|c|c|c|c|}
\hline $\begin{array}{l}\text { Sampling } \\
\text { site } \\
\text { identifier } \\
\text { (fig. 1) }\end{array}$ & $\begin{array}{l}\text { U.S. Geological } \\
\text { Survey identification } \\
\text { number }\end{array}$ & Site name & $\begin{array}{c}\text { Contributing } \\
\text { drainage area in } \\
\text { Johnson County } \\
\left(\mathrm{mi}^{2}\right)\end{array}$ & $\begin{array}{l}\text { Latitude } \\
\text { (degrees, minutes, } \\
\text { seconds) }\end{array}$ & $\begin{array}{l}\text { Longitude } \\
\text { (degrees, minutes, } \\
\text { seconds) }\end{array}$ & Sample type ${ }^{1}$ & $\begin{array}{l}\text { Contact } \\
\text { recreation } \\
\text { classification }\end{array}$ \\
\hline \multicolumn{8}{|c|}{ Mill Creek watershed } \\
\hline MI1 & 385356094491200 & Mill Creek at 127th Street & 4.4 & $38^{\circ} 53^{\prime} 57^{\prime \prime}$ & $94^{\circ} 49^{\prime} 12^{\prime \prime}$ & $1,2, \mathrm{~S}$ & $\mathrm{C}$ \\
\hline MI2 & 385357094491200 & $\begin{array}{l}\text { Discharge from Harold Street wastewater- } \\
\text { treatment facility }\end{array}$ & 0 & $38^{\circ} 53^{\prime} 57^{\prime \prime}$ & $94^{\circ} 49^{\prime} 23^{\prime \prime}$ & $1,2, \mathrm{~W}$ & $\mathrm{C}$ \\
\hline MI4 & 385800094485300 & Mill Creek at 87th Street Lane & 19.4 & $38^{\circ} 58^{\prime} 00^{\prime \prime}$ & $94^{\circ} 48^{\prime} 53^{\prime \prime}$ & $1,2, \mathrm{~S}$ & $\mathrm{C}$ \\
\hline MI5 & 390010094482100 & Little Mill Creek at Warwick Lane & 13.0 & $39^{\circ} 00^{\prime} 11^{\prime \prime}$ & $94^{\circ} 48^{\prime} 21^{\prime \prime}$ & 1,2 & B \\
\hline MI6 & 390058094493000 & Clear Creek at 63rd Street & 10.3 & $39^{\circ} 00^{\prime} 58^{\prime \prime}$ & $94^{\circ} 49^{\prime} 31^{\prime \prime}$ & 2 & $\mathrm{~B}$ \\
\hline MI7 & 06892513 & Mill Creek at Johnson Drive, Shawnee & 58.8 & $39^{\circ} 01^{\prime} 46^{\prime \prime}$ & $94^{\circ} 49^{\prime} 03^{\prime \prime}$ & $1,2, \mathrm{R}, \mathrm{S}$ & $\mathrm{C}$ \\
\hline \multicolumn{8}{|c|}{ Tomahawk Creek watershed } \\
\hline TO1 & 385238094411300 & Tomahawk Creek at Antioch Road & 10.3 & $38^{\circ} 52^{\prime} 38^{\prime \prime}$ & $94^{\circ} 41^{\prime} 13^{\prime \prime}$ & 1,2 & -- \\
\hline TO3 & 385550094371500 & $\begin{array}{l}\text { Discharge from Tomahawk Creek } \\
\text { wastewater-treatment facility }\end{array}$ & 0 & $38^{\circ} 55^{\prime} 50^{\prime \prime}$ & $94^{\circ} 37^{\prime} 15^{\prime \prime}$ & $1,2, \mathrm{~W}$ & -- \\
\hline \multicolumn{8}{|c|}{ Turkey Creek watershed } \\
\hline TU1 & 390027094415600 & Turkey Creek at 67th Street & 6.7 & $39^{\circ} 00^{\prime} 27^{\prime \prime}$ & $94^{\circ} 41^{\prime} 56^{\prime \prime}$ & $1,2, \mathrm{R}, \mathrm{S}$ & $\mathrm{B}$ \\
\hline TU2 & 390219094402000 & Turkey Creek at Highway 635 & 14.5 & $39^{\circ} 02 ’ 19^{\prime \prime}$ & $94^{\circ} 40^{\prime} 20^{\prime \prime}$ & 1,2 & $\mathrm{~B}$ \\
\hline TU3 & 390252094385400 & $\begin{array}{l}\text { Turkey Creek downstream of Myron } \\
\text { Nelson Complex wastewater-treatment } \\
\text { facility at Foxridge Road }\end{array}$ & 15.0 & $39^{\circ} 02^{\prime} 52^{\prime \prime}$ & $94^{\circ} 38^{\prime} 54^{\prime \prime}$ & $1,2, \mathrm{~W}$ & $\mathrm{~B}$ \\
\hline
\end{tabular}

${ }^{1}$ Sample type: 1 , indicates site was sampled during the first synoptic survey; 2 , indicates site was sampled during the second synoptic survey; $\mathrm{R}$, indicates a stormflow sampling site; $\mathrm{S}$, indicates a streambed-sediment sampling site; $\mathrm{W}$, indicates wastewater-treatment facility discharge.

${ }^{2}$ Contact recreation classification: B, indicates the primary contact recreational stream segment is by Kansas law or written permission of the landowner open to and accessible by the public; $\mathrm{b}$, indicates that the secondary contact recreational stream segment is not open to and accessible by the public under Kansas law; C, indicates that the primary contact recreation stream segment is not open to and accessible by the public under Kansas law; --, indicates capacity of the referenced stream segment to support the indicated beneficial use has not been determined by use attainability analysis (Kansas Department of Health and Environment, 2004c). 
Table 3. Wastewater-treatment facilities sampled in Johnson County, northeastern Kansas, their designed flow capacity, and secondary treatment processes.

[Information from E. Hack, Johnson County Wastewater and Public Works Department, written commun., 2003]

\begin{tabular}{lccl}
\hline \multicolumn{1}{c}{$\begin{array}{c}\text { Wastewater-treatment facility } \\
\text { (receiving stream) }\end{array}$} & $\begin{array}{c}\text { Discharge } \\
\text { sampling site } \\
\text { (fig. 1) }\end{array}$ & $\begin{array}{c}\text { Design flow } \\
\text { (million gallons per day) }\end{array}$ & Secondary treatment processes \\
\hline $\begin{array}{c}\text { Blue River Main Number 1 (Blue River } \\
\text { via Negro Creek) }\end{array}$ & BL6 & 3.0 & Extended aeration and activated sludge \\
$\begin{array}{l}\text { Cedar Creek (Cedar Creek) } \\
\begin{array}{l}\text { Indian Creek Middle Basin (Blue River } \\
\text { via Indian Creek) }\end{array}\end{array}$ & IN3 & 3.0 & Activated sludge \\
$\begin{array}{l}\text { Kill Creek (Kill Creek) } \\
\text { Harold Street (Mill Creek) }\end{array}$ & KI2 & 9.0 & Complete mix activated sludge \\
$\begin{array}{l}\text { Tomahawk Creek (Blue River via Indian } \\
\text { Creek) }\end{array}$ & MO3 & 2.5 & Activated sludge \\
Myron Nelson Complex (Turkey Creek) & TU3 & 3.2 & Trickling filter \\
${ }^{1}$ Mission Main & & 10.0 & Trickling filter \\
${ }^{1}$ Turkey Creek & & 15.0 & Trickling filter \\
\hline
\end{tabular}

${ }^{1}$ Mission Main and Turkey Creek facilities are two separate facilities with a common discharge to Turkey Creek; the two facilities combined are referred to as the Myron Nelson Complex.

north-flowing streams (Captain Creek, Cedar Creek, Kill Creek, and Mill Creek) are known to have steeper gradients and greater relief than the east- and south-flowing streams (O'Connor, 1971). Johnson County is underlain by Cambrian, Ordovician, Devonian, Mississippian, Pennsylvanian, and Quaternary sedimentary rock above a Precambrian basement. The sedimentary rock is characterized by alternating limestone and shale and minor amounts of fine-grained sandstone. Soils in the county generally consist of loess, glacial deposits, and residual from the weathering of bedrock (Plinsky and others, 1975).

The mean annual temperature from Olathe, Kansas (located approximately in the center of Johnson County), is $54.7^{\circ} \mathrm{F}$, with a mean monthly range of $28.0^{\circ} \mathrm{F}$ in January to $78.2^{\circ} \mathrm{F}$ in July (National Oceanic and Atmospheric Administration, 1966-98). Mean annual precipitation (1961-90) is 39.6 in., with 68 percent of the rain occurring during the growing season from April through September (National Oceanic and Atmospheric Administration, 1966-98).

Population increases in Johnson County have resulted in increased urban and suburban land uses. Since 1990, land parcels dedicated to residential and commercial land use in Johnson County have increased more than 45 percent (K. Skridulis, Johnson County Appraiser's Office, written commun., 2004). Figure 2 shows urban and nonurban land use for Johnson County in 2003; table 4 lists estimates of urban and nonurban land use and estimated number of septic systems per square mile by watershed (S. Porter, Johnson County Automated Information Mapping System, written commun., 2003). The "undeveloped" designation in table 4 includes agricultural land uses as well as vacant land. The "no data" land-use designation indicates land uses that could not be identified because they were not taxed by the county in 2003 . This designation includes the inactive Sunflower Army Ammunition Plant in the Captain and Kill Creek watersheds and the New Century AirCenter in the Cedar, Kill, and Little Bull Creek watersheds. The "no data" designation also includes most roads in the county. Impervious surface data were estimated by adding the total area of all buildings, courtyards, as well as paved and unpaved roads and parking lots in the county (S. Porter, Johnson County Automated Information Mapping System, written commun., 2003).

Brush Creek, Dykes Branch, Indian Creek, Rock Creek, Tomahawk Creek, and Turkey Creek watersheds contain the most urban development in the county, with more than 75 percent of these watersheds devoted to residential, commercial, industrial, and right-of-way land uses and less than 10 percent of each watershed identified as undeveloped (table 4). More than 18 percent of these watersheds are impervious surface. The Blue River and Mill Creek watersheds are experiencing the most-recent development (Mid-America Regional Council, 2002); both have more than 24 percent of their area devoted to residential, commercial, and industrial land uses. However, impervious surface areas in these watersheds are smaller than the more urbanized watersheds, and the largest percentage of land in each watershed is still undeveloped. The majority of land also is undeveloped in the Big Bull Creek, Captain Creek, Cedar Creek, Kill Creek and Little Bull Creek watersheds.

Assuming that the differences in the surface area of streams between watersheds are negligible, the percentage of land use devoted to surface water is largely a reflection of the size and number of surface-water impoundments within a watershed. The more area devoted to surface-water impoundments in a watershed, the larger the capacity of the watershed to 


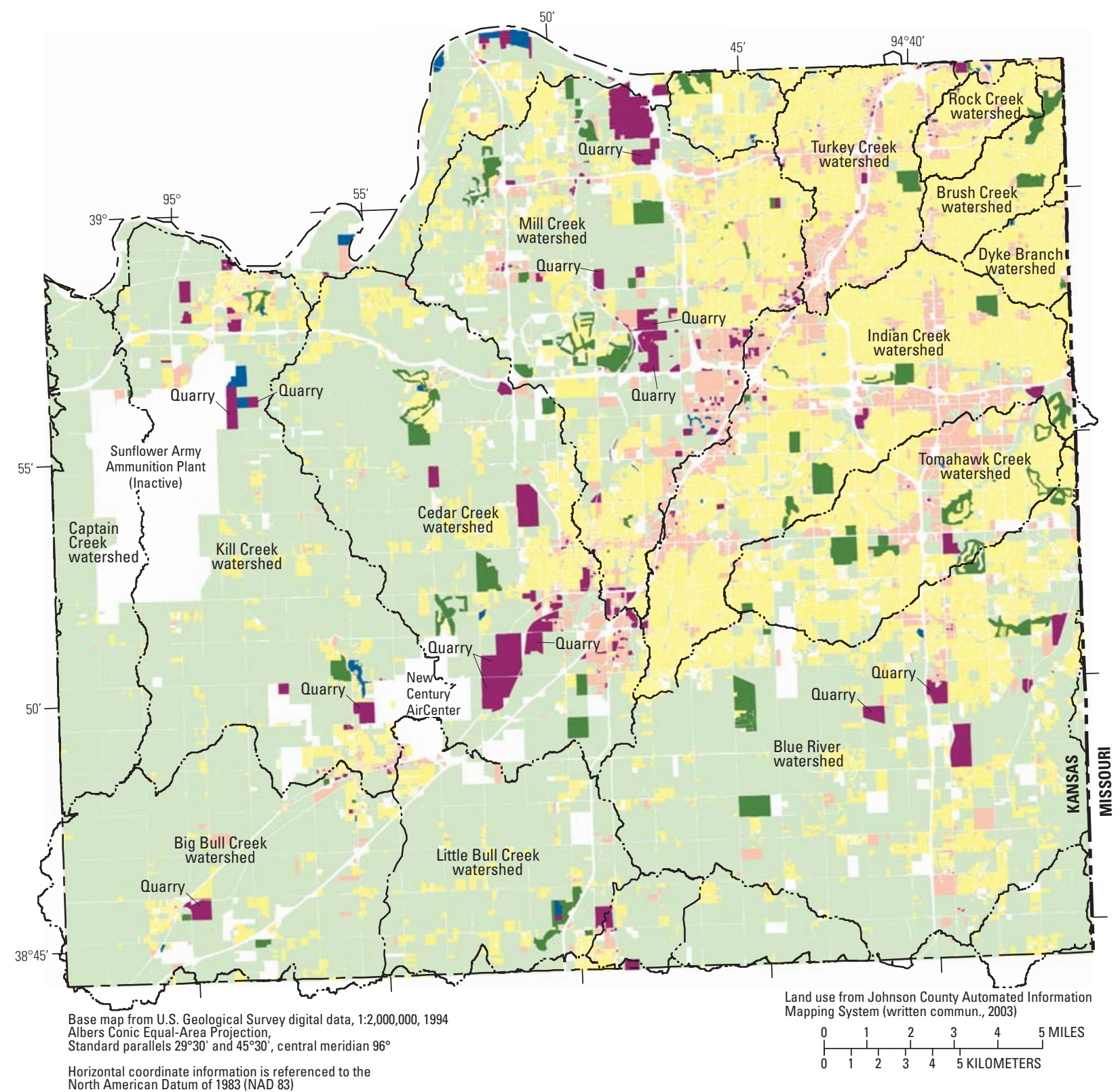

Horizontal coordinate information is referenced to the

EXPLANATION

Land use

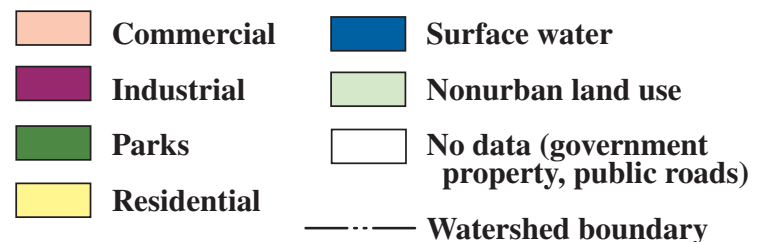

Figure 2. Urban and nonurban land use in Johnson County, northeast Kansas, 2003. 
Table 4. Estimates of urban and nonurban land-use percentages in selected watersheds of Johnson County, northeastern Kansas.

[Data from Johnson County Automated Information Mapping System, written commun., 2003; $\mathrm{mi}^{2}$, square miles]

\begin{tabular}{|c|c|c|c|c|c|c|c|c|c|c|c|}
\hline \multirow[b]{2}{*}{ Watershed (fig. 2) } & \multirow[b]{2}{*}{$\begin{array}{l}\text { Watershed } \\
\text { area }\left(\mathrm{mi}^{2}\right)\end{array}$} & \multicolumn{8}{|c|}{ Land-use percentage } & \multirow[b]{2}{*}{$\begin{array}{c}\text { Percentage } \\
\text { impervious } \\
\text { surface }\end{array}$} & \multirow{2}{*}{$\begin{array}{c}\text { Estimated } \\
\text { number of } \\
\text { septic } \\
\text { systems pe } \\
\text { square mile } \\
\text { of drainage } \\
\text { area }\end{array}$} \\
\hline & & Residential & Commercial & Industrial & $\begin{array}{l}\text { Right-of- } \\
\text { way }\end{array}$ & Parks & $\begin{array}{c}\text { Surface } \\
\text { water }\end{array}$ & $\begin{array}{c}\text { Undeveloped } \\
\text { (nonurban) }\end{array}$ & No data ${ }^{2}$ & & \\
\hline Big Bull Creek & 39.8 & 6.7 & 1.8 & 0.8 & 1.8 & 0.4 & 1.8 & 81.5 & 5.2 & 1.7 & 9.3 \\
\hline Blue River & 76.0 & 20.2 & 3.4 & 1.0 & .6 & 2.0 & 2.2 & 61.0 & 9.6 & 4.8 & 32.4 \\
\hline Brush Creek & 7.1 & 74.8 & 4.4 & 0 & .8 & 4.6 & .2 & 1.2 & 14.0 & 23.4 & 3.7 \\
\hline Captain Creek & 22.7 & 1.1 & .4 & 0 & .5 & 0 & 1.6 & 70.0 & 26.4 & 1.1 & 4.5 \\
\hline Cedar Creek & 59.0 & 12.4 & 4.9 & 3.8 & 2.5 & 2.8 & 2.3 & 64.5 & 6.8 & 5.0 & 16.6 \\
\hline Dykes Branch & 4.1 & 79.5 & 4.3 & 0 & .8 & 6 & .5 & 1.4 & 12.9 & 21.9 & 0 \\
\hline Indian Creek & 40.9 & 68.3 & 9.9 & .2 & 2.6 & 2.3 & .5 & 5.2 & 11.0 & 27.2 & 1.1 \\
\hline Kill Creek & 60.0 & 8.4 & 1.4 & 1.7 & 1.0 & 6 & 2.1 & 61.9 & 22.9 & 3.3 & 15.9 \\
\hline Rock Creek & 4.6 & 68.6 & 11.1 & .3 & 2.4 & 0 & .2 & .9 & 16.5 & 29.2 & .4 \\
\hline Tomahawk Creek & 23.4 & 67.8 & 7.4 & .4 & .8 & 2.9 & .8 & 8.5 & 11.4 & 18.9 & 13.8 \\
\hline Turkey Creek & 16.1 & 55.4 & 18.9 & .6 & 5.4 & 1.5 & .4 & 3.2 & 14.6 & 28.5 & 4.7 \\
\hline
\end{tabular}

1"Undeveloped" land use includes agricultural and vacant land.

2“No data" land use includes untaxed land uses (such as government property and public roads). 
store surface runoff before it enters a stream. These impoundments can slow streamflow response to runoff and may act as sinks for many sediment-associated water-quality contaminants. The most urban watersheds (Brush Creek, Dykes Branch, Indian Creek, Rock Creek, Tomahawk Creek, and Turkey Creek) have the fewest surface-water impoundments, totaling less than 1 percent of each watershed. Nonurban and urbanizing watersheds (Big Bull Creek, Blue River, Captain Creek, Cedar Creek, Kill Creek, Little Bull Creek, and Mill Creek) have the largest percentages of surface water.

Figure 3 shows agricultural and nonagricultural land use for 2003, and figure 4 shows the possible location of septic systems for 2003. The largest percentages of agricultural land are in the Blue River, Big Bull, Captain, Cedar, Kill, and Little Bull Creek watersheds (K. Skridulis, Johnson County Appraiser's Office, written commun., 2004). A greater percentage of land use in the southern Blue River watershed is devoted to agriculture than in the northern part of the watershed. Mill Creek has some agricultural land uses, whereas urban watersheds Brush, Dykes Branch, Indian, Rock, Tomahawk, and Turkey Creeks have little or no agricultural land uses. Possible septic system locations are based on county estimates of households and businesses not connected to sewage lines. The majority of septic systems are located in watersheds with predominantly nonurban land uses; the largest concentration of septic systems are in the Blue River watershed (table 4).

\section{Previous Investigations}

Although a comprehensive study of Johnson County streams has not been conducted, many streams and lakes within the individual watersheds have been studied. From 1982-83, a 13-month study of Cedar Lake and Lake Olathe (within the Cedar Creek watershed) was conducted by the city of Olathe. This study estimated sediment and nutrient loads contributed to each lake and found detectable concentrations of trace elements and pesticides in fish tissues (Lee and Sears, 1985). Subsequent studies conducted by Mau (2000) and Mau and others (2004) found that total nitrogen and phosphorus yields for the watershed upstream from Lake Olathe were consistent with watersheds of mixed agricultural land uses. Atrazine was found in the largest concentrations in spring and summer samples from the Cedar Creek watershed upstream from Lake Olathe, occasionally exceeding KDHE chronic aquatic-life criterion of $3.0 \mu \mathrm{g} / \mathrm{L}$ (Mau and others, 2004). The herbicides alachlor, atrazine, and metolachlor were detected in bottom sediment from both Cedar Lake and Lake Olathe (Mau, 2000).

USGS conducted a study of the Hillsdale Lake Basin (Johnson and Miami Counties) from May 1994 through May 1995, which sampled Big Bull and Little Bull Creeks in the southwestern part of Johnson County (Putnam, 1997). This study focused on the occurrence of nutrients and triazine herbicides in the basin during low-flow conditions. Samples from Big Bull Creek and Little Bull Creek at times exceeded the 10-mg/L Maximum Contaminant Level (MCL) for total nitrite plus nitrate in drinking water (U.S. Environmental Protection Agency, 2002) and routinely exceeded the Hillsdale WaterQuality Protection Project proposed total phosphorus criterion of $0.05 \mathrm{mg} / \mathrm{L}$. The largest concentrations of total nitrogen and total phosphorus occurred directly downstream from WWTFs located in the Big Bull and Little Bull Creek Basins and decreased downstream. Additionally, triazine herbicide samples exceeded the USEPA atrazine MCL of $3.0 \mu \mathrm{g} / \mathrm{L}$ in 17 of 36 samples from Big Bull Creek and in 6 of 33 samples from Little Bull Creek. Triazine herbicides were detected more frequently in the spring after herbicide application (Putnam, 1997).

USGS also studied water quality in stormwater runoff in the Blue River Basin of Kansas and Missouri at sites with varying degrees of urbanization from 1981 to 1982 (Blevins, 1986). Highly urbanized sites in the lower Blue River Basin in Kansas City, Missouri, recorded as much as three times the stormwater runoff per unit area as less urbanized sites in the basin. Highly urbanized sites with concrete channels in the Brush Creek Basin generally had smaller concentrations of suspended sediment, nutrients, and metals than upstream sites, but suspendedsediment concentrations increased when stream water reached unlined channels. The largest concentrations of suspended sediment, nitrogen, phosphorus, and most metals were found in the Blue River Basin downstream from agricultural land uses. However, the initial flush of runoff from urban areas generally had larger concentrations of biological oxygen demand, suspended sediment, dissolved solids, as well as nutrients and metals, due to flushing of combined sewers (Blevins, 1986).

The Blue River and Brush Creek watersheds also were studied from 1998 through 2000, and water samples were analyzed for major ions, nutrients, bacteria, pesticides, wastewaterindicator compounds, and selected pharmaceuticals (Wilkison and others, 2002). Continuous discharge of wastewater effluent was the primary source of nutrients, wastewater-indicator compounds, and pharmaceutical compounds in stream samples in the Blue River Basin. Wastewater inputs into Brush Creek were a result of stormflow that triggered the overflow of combined and sanitary sewers, discharging untreated wastewater into the creek. During base-flow conditions the Blue River contained substantially larger nutrient and wastewater compound concentrations, as well as median Escherichia coli (E. coli) bacteria densities that were double that of Brush Creek samples. However, during some stormflow conditions, wastewater compounds were larger in Brush Creek samples (Wilkison and others, 2002).

A study was conducted in the Indian and Rock Creek watersheds during 1981-82 through the National Urban Runoff Program funded by USEPA (Mid-America Regional Council and F.X. Browne and Associates, Inc., 1983). This study found that urban areas with more commercial and industrial areas and high traffic volumes caused increased contributions of nitrogen, oxygen-demanding substances, and metals including copper, zinc, and lead. Suspended-sediment concentrations were much larger during stormflow conditions; nonpoint sources of sediment were thought to be from exposed soil and from basins with 


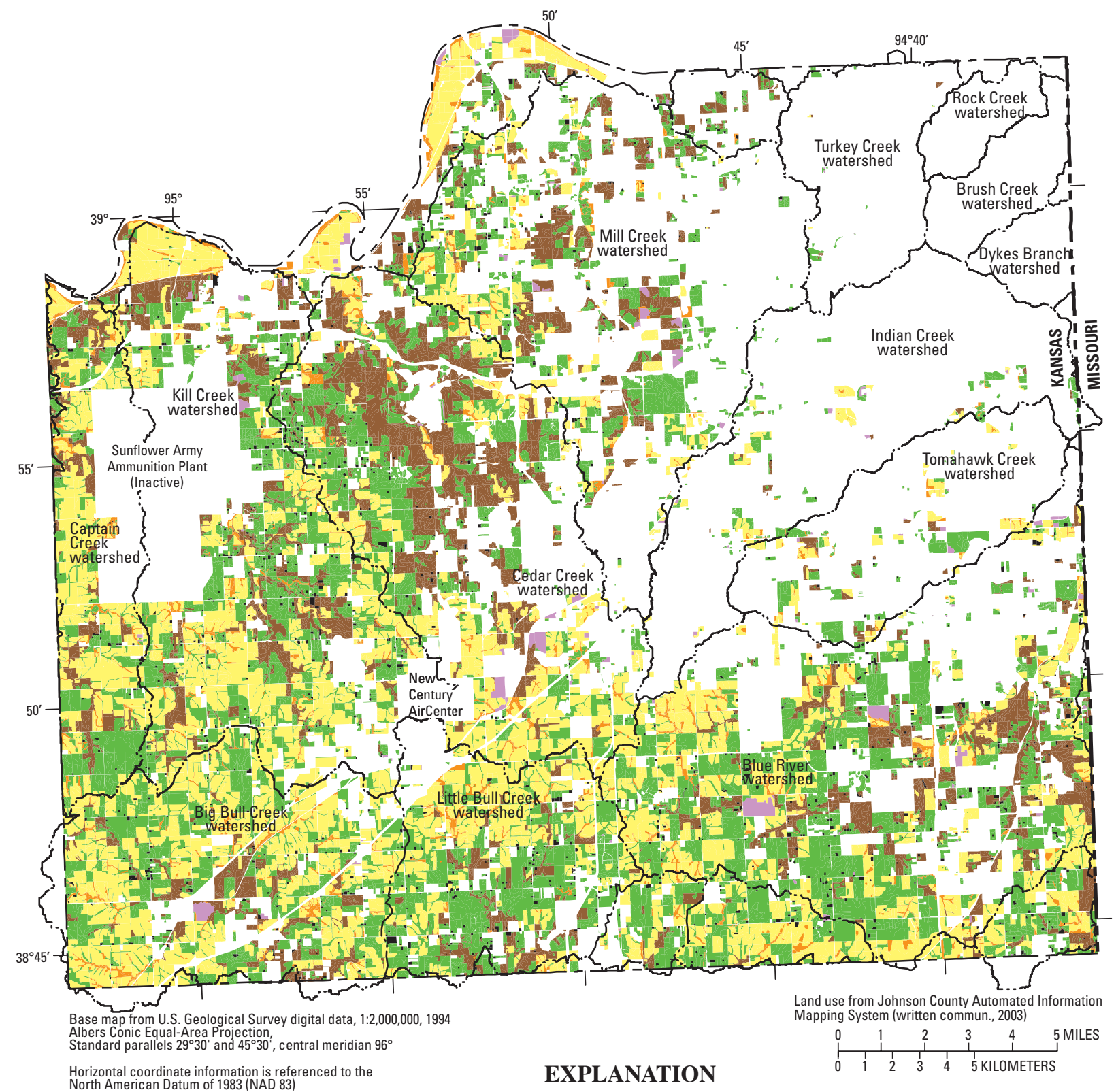

Land use

\begin{tabular}{|c|c|}
\hline Homesite & Canopy cover \\
\hline \multirow{2}{*}{$\begin{array}{l}\text { Commercial agricultural } \\
\text { use-Nurseries, tree farms, } \\
\text { and so forth }\end{array}$} & Nonusable acreage \\
\hline & Nonagricultural land \\
\hline Cropland & \\
\hline Grassland & \\
\hline
\end{tabular}

Figure 3. Agricultural and nonagricultural land use in Johnson County, northeastern Kansas, 2003. 


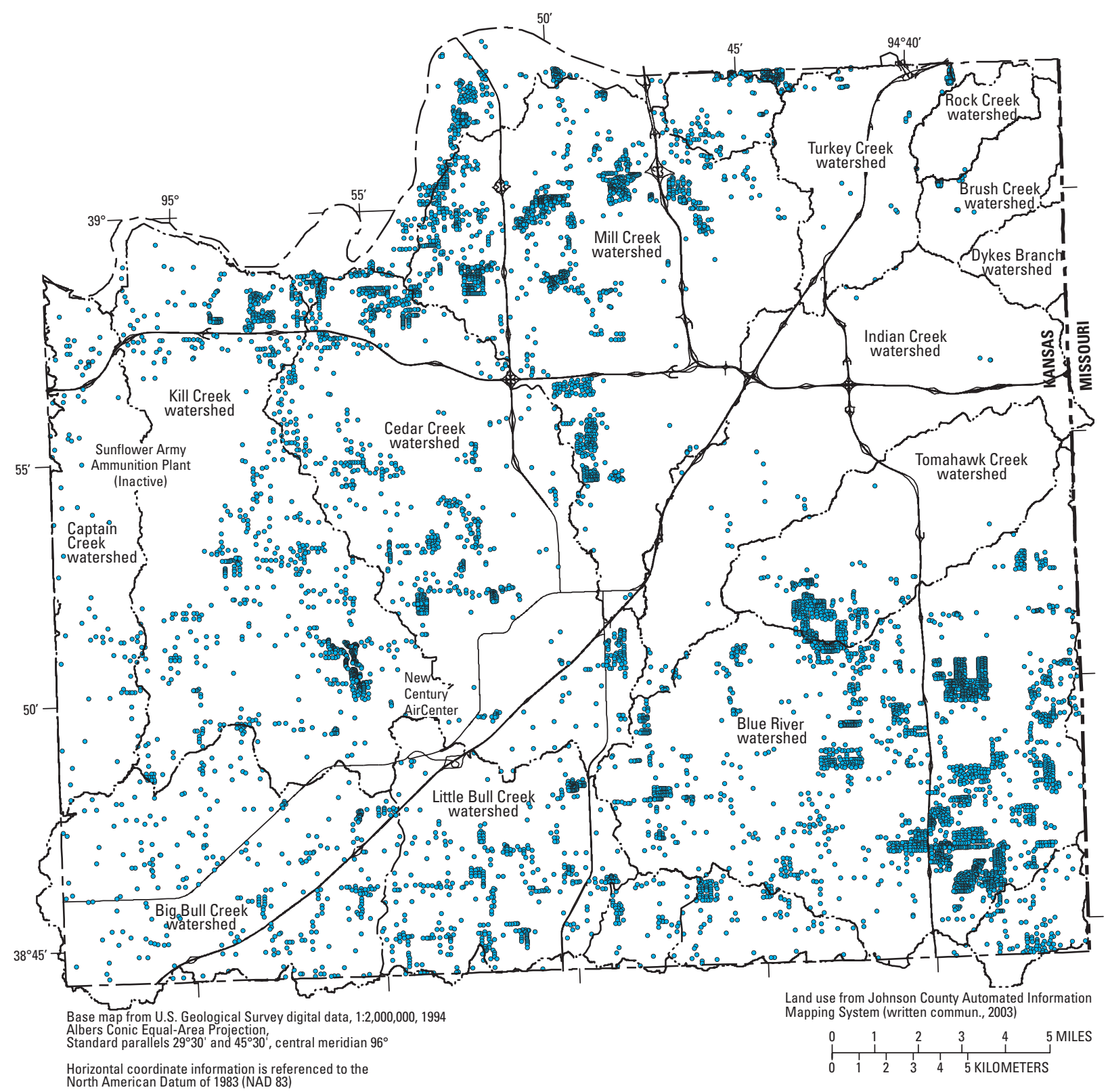

EXPLANATION

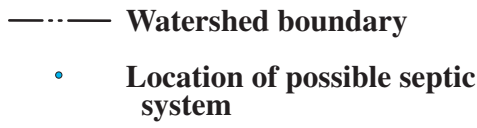

Figure 4. Possible septic system locations, Johnson County, northeastern Kansas, 2003. 
steep channel slopes. Contaminants such as phosphorus, manganese, and iron were found to be associated with suspendedsediment concentrations (Mid-America Regional Council and F.X. Browne and Associates, Inc., 1983).

The USGS National Water-Quality Assessment (NAWQA) Program compiled historical data from surfacewater sites with a minimum of 25 samples throughout the United States from 1980 to 1990 in basins with varying land uses (U.S. Geological Survey, 2004). Along with other data, water samples were analyzed for nutrients, including ammonia, nitrate, total nitrogen, total phosphorus, and orthophosphorus. Nutrient data are presented on a Web site (http://water.usgs.gov/nawqa/nutrients/datasets/ retro.data.sw) and are used in this report to compare national conditions with those in Johnson County stream-water samples. The NAWQA Program also completed assessments in 51 study units from 1992 to 2001 and analyzed water samples for the occurrence of pesticide compounds (Martin and others, 2003). Pesticide data are summarized in Martin and others (2003) and on a Web site (http://water.usgs.gov/nawqa/) and are used periodically in this report to compare national conditions with those in Johnson County stream-water samples collected from October 2002 through June 2004.

Wastewater and pharmaceutical compounds have been of increasing interest in surface water and streambed sediment in recent years; however, there are few established criteria or guidelines for these compounds. To place wastewater and pharmaceutical compound concentrations found in Johnson County stream water in context with concentrations in other parts of the United States, two USGS studies performed by Kolpin and others (2002) and Lee and others (2004) were used for comparison purposes. Kolpin and others (2002) sampled streams for wastewater and pharmaceutical compounds on a national scale to determine occurrence in a variety of geographic areas and land uses. However, unlike some samples collected as part of the study described in this report, Kolpin and others (2002) did not directly sample WWTF discharges; therefore, their results may be underrepresentative compared to the stream sample results presented in this report. Lee and others (2004) sampled 65 sites in Minnesota, including WWTF influent, landfill and feedlot lagoon leachate, surface water, ground water, and WWTF discharges. Both of these studies analyzed wastewater and pharmaceutical compounds from whole-water samples (this study used filtered water samples). Lee and others (2004) observed that whole and filtered-water analyses found similar concentrations for most wastewater and pharmaceutical compounds; however, some compounds had smaller concentrations in filtered-water analyses due to adsorption to suspended sediment. For this reason, these studies may report larger concentrations of some contaminants than the Johnson County study reported herein due to the inclusion of both sediment-bound and dissolved contaminants.

\section{Methods}

Sampling sites were selected throughout Johnson County (fig. 1) to: (1) provide spatial coverage of the county and (2) differentiate point and nonpoint sources of contaminants in each watershed.

\section{Base-Flow Samples}

Synoptic, stream-water samples were collected during November 4-7, 2000, and July 14-18, 2003, to provide two "snapshots" of Johnson County streams during base-flow hydrologic conditions. The base-flow data were used to determine spatial variation in Johnson County streams and to contrast water-quality conditions upstream and downstream from WWTFs as well as to compare with water-quality samples collected during stormflow conditions. Although a maximum of two base-flow samples from each site do not provide a statistical basis for evaluating stream-water-quality conditions at any given site, they do give an indication of the sources and occurrence of water-quality contaminants during base-flow conditions. Base flow is defined as the sustained low flow of a stream originating primarily from springs or ground-water seepage or from point-source discharges such as WWTFs. Synoptic samples were collected such that sites within a particular watershed were sampled on the same day and that all sites in the county were sampled during a 1-week period. This was done to capture consistent hydrologic conditions throughout the sampling period. Streamflow was measured using methods presented in Buchanan and Somers (1969).

Although 45 sites throughout Johnson County were selected initially for base-flow sampling, 12 sites did not have measurable streamflow during the November 4-7, 2002, synoptic survey, and 3 sites lacked streamflow during the July 14-18, 2003, synoptic survey. At the majority of sites sampled, riffles were less than $0.5 \mathrm{ft}$ in depth, and grab samples for suspended sediment, dissolved solids, major ions, nutrients, and trace elements were collected using equal-width increments (EWI) (Wilde and others, 1998). Cross-section measurements of specific conductance, $\mathrm{pH}$, water temperature, turbidity, and dissolved oxygen were made onsite using Yellow Springs Instruments (YSI, Yellow Springs, Ohio) 6600 multiparameter water-quality monitors. Values were recorded as a mean of cross-sectional measurements, and streams were generally well mixed. Air temperature and barometric pressure also were recorded at the time of sampling. Samples for analysis of bacteria, pesticides, and wastewater and pharmaceutical compounds were collected by grab sample at the centroid of flow. Samples were collected in accordance with USGS protocols for the collection of wastewater and pharmaceutical compounds with the exception of the final methanol rinse (Lewis and Zaugg, 2003). 


\section{Stormflow Samples}

Stormflow samples were collected from October 2002 through June 2004 during or soon after precipitation to characterize nonpoint sources of contamination associated with runoff conditions. Three stormflow samples were collected from each of the following sites: BL5 on the Blue River, CE6 on Cedar Creek, IN6 on Indian Creek, KI6b on Kill Creek, MI7 on Mill Creek, and TU1 on Turkey Creek (fig. 1, table 2). Additional stream-water samples were collected from sites BL5, CE6, IN6, KI6b, and MI7 and included in this report to improve definition of water quality during varying streamflow conditions.

Discrete stormflow samples generally were collected during streamflow conditions at least one and as large as four orders of magnitude greater than that observed during baseflow sampling. EWI sampling methods (Wilde and others, 1998) were used when possible to collect samples; otherwise, dip samples from the centroid of flow were collected using a weighted bottle, or samples were collected with an automated sampler. Cross-sectional water-quality monitor readings recorded at the same time as stormflow sample collection indicated that streamflow conditions at sites generally were well mixed. Four samples were collected concurrently to compare point (dip or automated sampler) and EWI sampling methods. These samples are discussed in the "Quality Assurance and Quality Control" section. Stormflow sampling sites are listed with their estimated corresponding drainage areas, percentage land use, and estimated number of septic systems per square mile (table 5).

\section{Streambed-Sediment Samples}

Streambed-sediment samples were collected from March 31 through April 3, 2003, at 15 sites within the Big Bull Creek, Blue River, Captain Creek, Cedar Creek, Indian Creek, Kill Creek, Mill Creek, Tomahawk Creek, and Turkey Creek watersheds (table 2). Eight of the 15 sites were located downstream from continuously discharging municipal WWTFs. These samples were used to determine contaminant source areas, to evaluate fate and transport of potential contaminants, and to estimate toxicity of streambed sediments. A variety of chemical constituents often occur in larger concentrations in streambed sediment than in the overlying water column (Van Donsel and Geldreich, 1971; Horowitz, 1991). Contaminated sediment can be toxic to benthic organisms and the contaminants can bioaccumulate in fish, insects, and mammals (U.S. Environmental Protection Agency, 2000a). Sediment samples were collected during a period of low flow using stainless-steel and plastic spoons. The bottom sediment was removed from the stream, placed in plastic or glass containers, homogenized, and shipped for analysis.

\section{Sample Analysis}

Base- and stormflow samples were analyzed for suspended sediment, dissolved solids, major ions, nutrients (nitrogen and phosphorus), trace elements, bacteria, and pesticide, wastewater, and pharmaceutical compounds. Streambed-sediment samples were analyzed for total organic carbon, total carbon, major ions, nutrients, trace elements, bacteria, and pesticide, wastewater, and pharmaceutical compounds. Constituents analyzed in all stream-water and streambed-sediment samples in this study are listed in table 6. Complete analytical results of base- and stormflow surface-water samples and streambedsediment samples are posted on a USGS Web site (http://ks.water.usgs.gov/Kansas/studies/qw/joco/) and are on file with USGS in Lawrence, Kansas.

Water samples were analyzed at several laboratories. Suspended-sediment samples were analyzed at the USGS Sediment Laboratory in Iowa City, Iowa, according to methods presented in Guy (1969). Major ions, nutrients, and fecal coliform bacteria were analyzed at the Johnson County Environmental Laboratory in Johnson County, Kansas, according to standard methods (American Public Health Association and others, 1995), and selected replicate samples were sent to the USGS National Water-Quality Laboratory (NWQL) in Denver, Colorado, and analyzed according to methods presented in Fishman and Friedman (1989), Faires (1993), and Fishman (1993). Analysis of water samples for $E$. coli and enterococci bacteria was performed at the USGS laboratory in Lawrence, Kansas. These samples were processed within 6 hours of collection using membrane filtration methods described by Wilde and others (1998).

Water samples for selected dissolved pesticides and wastewater compounds were analyzed at NWQL using methods described by Zaugg and others $(1995,2002)$. Pharmaceutical compounds in filtered samples were analyzed at NWQL using methods described by Cahill and others (2004). These methods are described as "information rich" because of their ability to report values at very small concentrations, often less than the microgram-per-liter level. These reported values may be denoted as estimated (E) for some constituents when values are reported outside of instrument calibration range or when concentrations are reported at less than NWQL laboratory reporting levels (Childress and others, 1999).

Chemical analysis of streambed sediment was performed at the Atlanta, Georgia, USGS sediment chemistry laboratory using digestion after homogenization and passage through a $63-\mu \mathrm{m}$ sieve (Horowitz and others, 2001). Bacteria in streambed sediment was determined at the Columbus, Ohio, USGS microbiology laboratory 1 day after sample collection using methods described by Francy and Darner (1998). Pesticides in streambed sediment were determined by dual-capillary column gas chromatography with electron capture detection (Foreman and others, 1995). Wastewater compounds (which include some compounds classified as pesticides in this report) in streambed sediment were extracted using accelerated solvent extraction (ASE) with 50-percent isopropanol at two 
Table 5. Stormflow sampling sites, contributing drainage areas, estimates of urban and nonurban land-use percentages, and estimated number of septic systems per square mile of drainage area in Johnson County, northeastern Kansas.

[[Data from Johnson County Automated Information Mapping System, written commun., 2003; $\mathrm{mi}^{2}$, square miles]

\begin{tabular}{|c|c|c|c|c|c|c|c|c|c|c|c|}
\hline \multirow{2}{*}{$\begin{array}{l}\text { Stormflow } \\
\text { sampling site } \\
\text { identifier } \\
\text { (fig. 1) }\end{array}$} & \multirow{2}{*}{$\begin{array}{l}\text { Contributing } \\
\text { drainage } \\
\text { area }\left(\mathrm{mi}^{2}\right)\end{array}$} & \multicolumn{8}{|c|}{ Percentage land use } & \multirow{2}{*}{$\begin{array}{c}\text { Percentage } \\
\text { impervious } \\
\text { surface }\end{array}$} & \multirow{2}{*}{$\begin{array}{c}\text { Estimated } \\
\text { number of } \\
\text { septic systems } \\
\text { per square mile } \\
\text { of drainage } \\
\text { area }\end{array}$} \\
\hline & & Residential & Commercial & Industrial & $\begin{array}{l}\text { Right-of- } \\
\text { way }\end{array}$ & Parks & $\begin{array}{c}\text { Surface } \\
\text { water }\end{array}$ & $\begin{array}{c}\text { Un- } \\
\text { developed }^{1} \\
\text { (nonurban) }\end{array}$ & No data ${ }^{2}$ & & \\
\hline BL5 & 65.7 & 15.1 & 2.5 & 1.0 & 0.6 & 1.6 & 2.2 & 69.3 & 7.7 & 3.0 & 36.5 \\
\hline CE6 & 58.5 & 12.4 & 4.9 & 3.8 & 2.5 & 2.8 & 2.3 & 64.5 & 6.8 & 3.9 & 16.6 \\
\hline IN6 & 63.1 & 68.1 & 8.9 & .3 & 1.9 & 2.2 & .6 & 6.4 & 11.6 & 23.5 & 5.7 \\
\hline KI6b & 48.6 & 6.4 & .9 & 1.3 & .8 & .5 & 1.9 & 61.7 & 26.5 & 2.9 & 14.8 \\
\hline MI7 & 58.8 & 26.7 & 11.1 & 2.8 & 3.4 & 3.4 & 1.9 & 37.8 & 12.9 & 12.2 & 24.4 \\
\hline TU1 & 6.7 & 48.2 & 26.7 & .4 & 5.2 & 0 & .5 & 2.8 & 16.2 & 30.9 & 1.1 \\
\hline
\end{tabular}

1"Undeveloped" land use includes agricultural and vacant land.

2“No data" land use includes untaxed land uses (such as government property and public roads). 
Table 6. Constituents analyzed in stream-water and streambed-sediment samples collected from selected Johnson County streams, northeastern Kansas, October 2002-June 2004.

[X, analyzed; --, not analyzed or not applicable; N/A, no laboratory reporting level established; <, less than; E, estimated; $\mu$ S/cm, microsiemens per centimeter at 25 degrees Celsius; FNU, formazin nephelometric units; mg/L, milligrams per liter; mg/kg, milligrams per kilogram; $\mu \mathrm{g} / \mathrm{L}$, micrograms per liter; $\mu \mathrm{g} / \mathrm{kg}$, micrograms per kilogram; col/100 mL, colonies per 100 milliliters]

\begin{tabular}{|c|c|c|c|c|c|}
\hline Constituent & $\begin{array}{l}\text { Base-flow } \\
\text { samples }\end{array}$ & $\begin{array}{l}\text { Stormflow } \\
\text { samples }\end{array}$ & $\begin{array}{l}\text { Streambed- } \\
\text { sediment } \\
\text { sample }\end{array}$ & $\begin{array}{c}\text { Stream-water } \\
\text { laboratory reporting } \\
\text { level }\end{array}$ & $\begin{array}{c}\text { Streambed- } \\
\text { sediment } \\
\text { laboratory } \\
\text { reporting level }\end{array}$ \\
\hline Specific conductance & $\mathrm{X}$ & $\mathrm{X}$ & -- & $1 \mu \mathrm{S} / \mathrm{cm}$ & -- \\
\hline $\mathrm{pH}$ & $\mathrm{X}$ & $\mathrm{X}$ & -- & 0.1 standard units & -- \\
\hline Water temperature & $\mathrm{X}$ & $\mathrm{X}$ & -- & 0.1 degrees Celsius & -- \\
\hline Dissolved oxygen & $\mathrm{X}$ & $\mathrm{X}$ & -- & $0.1 \mathrm{mg} / \mathrm{L}$ & -- \\
\hline Turbidity & $\mathrm{X}$ & $\mathrm{X}$ & -- & $0.1 \mathrm{FNU}$ & -- \\
\hline Alkalinity & $\mathrm{X}$ & $\mathrm{X}$ & -- & $2 \mathrm{mg} / \mathrm{L}$ & -- \\
\hline Total organic carbon & -- & -- & $\mathrm{X}$ & -- & $1,000 \mathrm{mg} / \mathrm{kg}$ \\
\hline Total carbon & -- & -- & $X$ & -- & $1,000 \mathrm{mg} / \mathrm{kg}$ \\
\hline Suspended sediment & $\mathrm{X}$ & $\mathrm{X}$ & -- & $1 \mathrm{mg} / \mathrm{L}$ & -- \\
\hline Dissolved solids & $\mathrm{X}$ & $X$ & -- & $2 \mathrm{mg} / \mathrm{L}$ & -- \\
\hline \multicolumn{6}{|c|}{ Major lons } \\
\hline Calcium & $\mathrm{X}$ & $\mathrm{X}$ & $\mathrm{X}$ & $.10 \mathrm{mg} / \mathrm{L}$ & $1,000 \mathrm{mg} / \mathrm{kg}$ \\
\hline Magnesium & $\mathrm{X}$ & $\mathrm{X}$ & $\mathrm{X}$ & $.10 \mathrm{mg} / \mathrm{L}$ & $1,000 \mathrm{mg} / \mathrm{kg}$ \\
\hline Sodium & $\mathrm{X}$ & $\mathrm{X}$ & $\mathrm{X}$ & $.10 \mathrm{mg} / \mathrm{L}$ & $1,000 \mathrm{mg} / \mathrm{kg}$ \\
\hline Potassium & $\mathrm{X}$ & $\mathrm{X}$ & $\mathrm{X}$ & $.10 \mathrm{mg} / \mathrm{L}$ & $1,000 \mathrm{mg} / \mathrm{kg}$ \\
\hline Sulfate & $\mathrm{X}$ & $\mathrm{X}$ & -- & $.05 \mathrm{mg} / \mathrm{L}$ & -- \\
\hline Chloride & $\mathrm{X}$ & $\mathrm{X}$ & -- & $10 \mathrm{mg} / \mathrm{L}$ & -- \\
\hline Fluoride & $X$ & $X$ & -- & $.10 \mathrm{mg} / \mathrm{L}$ & -- \\
\hline Silica & $\mathrm{X}$ & $\mathrm{X}$ & -- & $.10 \mathrm{mg} / \mathrm{L}$ & -- \\
\hline \multicolumn{6}{|c|}{ Nutrients } \\
\hline Nitrogen, total, as $\mathrm{N}$ & -- & -- & $\mathrm{X}$ & & $100 \mathrm{mg} / \mathrm{kg}$ \\
\hline Nitrogen, nitrite, as $\mathrm{N}$ & $\mathrm{X}$ & $\mathrm{X}$ & -- & $.02 \mathrm{mg} / \mathrm{L}$ & -- \\
\hline Nitrogen, nitrite plus nitrate, as $\mathrm{N}$ & $\mathrm{X}$ & $\mathrm{X}$ & -- & $.10 \mathrm{mg} / \mathrm{L}$ & -- \\
\hline Nitrogen, ammonia, as $\mathrm{N}$ & $\mathrm{X}$ & $\mathrm{X}$ & -- & $.02 \mathrm{mg} / \mathrm{L}$ & -- \\
\hline $\begin{array}{l}\text { Nitrogen, ammonia plus dissolved organic nitro- } \\
\text { gen, as } \mathrm{N}\end{array}$ & $\mathrm{X}$ & $\mathrm{X}$ & -- & $.20 \mathrm{mg} / \mathrm{L}$ & -- \\
\hline Nitrogen ammonia plus total organic nitrogen, as $\mathrm{N}$ & $\mathrm{X}$ & $\mathrm{X}$ & & $.20 \mathrm{mg} / \mathrm{L}$ & \\
\hline Phophorus, total & $\mathrm{X}$ & $\mathrm{X}$ & $\mathrm{X}$ & $.01 \mathrm{mg} / \mathrm{L}$ & $100 \mathrm{mg} / \mathrm{kg}$ \\
\hline Phosphorus, orthophosphate, as $\mathrm{P}$ & $\mathrm{X}$ & $\mathrm{X}$ & -- & $.01 \mathrm{mg} / \mathrm{L}$ & -- \\
\hline Phosphorus, dissolved & $\mathrm{X}$ & $\mathrm{X}$ & -- & $.01 \mathrm{mg} / \mathrm{L}$ & -- \\
\hline \multicolumn{6}{|c|}{ Trace elements } \\
\hline Aluminum & -- & -- & $\mathrm{X}$ & -- & $1 \mathrm{mg} / \mathrm{kg}$ \\
\hline Antimony & -- & -- & $X$ & -- & $.10 \mathrm{mg} / \mathrm{kg}$ \\
\hline Arsenic & -- & -- & $X$ & -- & $.10 \mathrm{mg} / \mathrm{kg}$ \\
\hline Barium & -- & -- & $X$ & -- & $1 \mathrm{mg} / \mathrm{kg}$ \\
\hline Beryillium & -- & -- & $X$ & -- & $.10 \mathrm{mg} / \mathrm{kg}$ \\
\hline Boron & -- & -- & $X$ & -- & $1 \mathrm{mg} / \mathrm{kg}$ \\
\hline
\end{tabular}


Table 6. Constituents analyzed in surface-water and streambed-sediment samples collected from selected Johnson County streams, northeastern Kansas, October 2002-June 2004.-Continued

$[\mathrm{X}$, analyzed; --, not analyzed or not applicable; N/A, no laboratory reporting level established; <, less than; E, estimated; $\mu \mathrm{S} / \mathrm{cm}$, microsiemens per centimeter at 25 degrees Celsius; FNU, formazin nephelometric units; mg/L, milligrams per liter; mg/kg, milligrams per kilogram; $\mu \mathrm{g} / \mathrm{L}$, micrograms per liter; $\mu \mathrm{g} / \mathrm{kg}$, micrograms per kilogram; col/100 mL, colonies per 100 milliliters]

\begin{tabular}{|c|c|c|c|c|c|}
\hline Constituent & $\begin{array}{c}\text { Base-flow } \\
\text { samples }\end{array}$ & $\begin{array}{l}\text { Stormflow } \\
\text { samples }\end{array}$ & $\begin{array}{l}\text { Streambed- } \\
\text { sediment } \\
\text { sample }\end{array}$ & $\begin{array}{c}\text { Stream-water } \\
\text { laboratory reporting } \\
\text { level }\end{array}$ & $\begin{array}{l}\text { Streambed- } \\
\text { sediment } \\
\text { laboratory } \\
\text { reporting level }\end{array}$ \\
\hline \multicolumn{6}{|c|}{ Trace elements-Continued } \\
\hline Cadmium & -- & -- & $X$ & -- & $0.1 \mathrm{mg} / \mathrm{kg}$ \\
\hline Chromium & -- & -- & $X$ & -- & $1 \mathrm{mg} / \mathrm{kg}$ \\
\hline Cobalt & -- & -- & $\mathrm{X}$ & -- & $1 \mathrm{mg} / \mathrm{kg}$ \\
\hline Copper & -- & -- & $X$ & -- & $1 \mathrm{mg} / \mathrm{kg}$ \\
\hline Iron & $\mathrm{X}$ & $X$ & $X$ & $0.01 \mathrm{mg} / \mathrm{L}$ & $1,000 \mathrm{mg} / \mathrm{kg}$ \\
\hline Lead & -- & -- & $\mathrm{X}$ & -- & $1 \mathrm{mg} / \mathrm{kg}$ \\
\hline Lithium & -- & -- & $\mathrm{X}$ & -- & $1 \mathrm{mg} / \mathrm{kg}$ \\
\hline Manganese & $X$ & $X$ & $X$ & $.01 \mathrm{mg} / \mathrm{L}$ & $10 \mathrm{mg} / \mathrm{kg}$ \\
\hline Mercury & -- & -- & $X$ & -- & $.01 \mathrm{mg} / \mathrm{kg}$ \\
\hline Molybdenum & -- & -- & $X$ & -- & $1 \mathrm{mg} / \mathrm{kg}$ \\
\hline Nickel & -- & -- & $\mathrm{X}$ & -- & $1 \mathrm{mg} / \mathrm{kg}$ \\
\hline Selenium & -- & -- & $\mathrm{X}$ & -- & $.1 \mathrm{mg} / \mathrm{kg}$ \\
\hline Silver & -- & -- & $\mathrm{X}$ & -- & $.5 \mathrm{mg} / \mathrm{kg}$ \\
\hline Strontium & -- & -- & $\mathrm{X}$ & -- & $1 \mathrm{mg} / \mathrm{kg}$ \\
\hline Sulfur & -- & -- & $\mathrm{X}$ & -- & $1,000 \mathrm{mg} / \mathrm{kg}$ \\
\hline Titanium & -- & -- & $\mathrm{X}$ & -- & $50 \mathrm{mg} / \mathrm{kg}$ \\
\hline Uranium & -- & -- & $\mathrm{X}$ & -- & $.05 \mathrm{mg} / \mathrm{kg}$ \\
\hline Vanadium & & & & & $1 \mathrm{mg} / \mathrm{kg}$ \\
\hline Zinc & -- & -- & $X$ & -- & $1 \mathrm{mg} / \mathrm{kg}$ \\
\hline \multicolumn{6}{|c|}{ Bacteria } \\
\hline Fecal coliform & $\mathrm{X}$ & $\mathrm{X}$ & $X$ & $10 \mathrm{col} / 100 \mathrm{~mL}$ & N/A \\
\hline Escherichia coli & $\mathrm{X}$ & $X$ & $X$ & $1 \mathrm{col} / 100 \mathrm{~mL}$ & N/A \\
\hline Enterococci & $\mathrm{X}$ & $\mathrm{X}$ & $\mathrm{X}$ & $1 \mathrm{col} / 100 \mathrm{~mL}$ & N/A \\
\hline \multicolumn{6}{|c|}{ Pesticide compounds (filtered water analysis) } \\
\hline 1-Napthol & $\mathrm{X}$ & $\mathrm{X}$ & -- & $.088 \mu \mathrm{g} / \mathrm{L}$ & -- \\
\hline 2,6-Diethylaniline & $\mathrm{X}$ & $\mathrm{X}$ & -- & $.006 \mu \mathrm{g} / \mathrm{L}$ & -- \\
\hline 2-[(2-Ethyl-6-methylphenyl)amino]-1-propanol & $\mathrm{X}$ & $\mathrm{X}$ & -- & $.12 \mu \mathrm{g} / \mathrm{L}$ & -- \\
\hline 2-Chloro-4-isopropylamino-6-amino-s-triazine & $\mathrm{X}$ & $\mathrm{X}$ & -- & $.006 \mu \mathrm{g} / \mathrm{L}$ & -- \\
\hline 2-Chloro-2,6-diethylacetanilide & $\mathrm{X}$ & $\mathrm{X}$ & -- & $.005 \mu \mathrm{g} / \mathrm{L}$ & -- \\
\hline 2-Ethyl-6-methylaniline & $\mathrm{X}$ & $\mathrm{X}$ & -- & $.0045 \mu \mathrm{g} / \mathrm{L}$ & -- \\
\hline 3,4-Dichloroaniline & $\mathrm{X}$ & $\mathrm{X}$ & -- & $.0045 \mu \mathrm{g} / \mathrm{L}$ & -- \\
\hline 4-Chloro-2-methylphenol & $\mathrm{X}$ & $\mathrm{X}$ & -- & $.0056 \mu \mathrm{g} / \mathrm{L}$ & -- \\
\hline Acetochlor & $\mathrm{X}$ & $\mathrm{X}$ & -- & $.006 \mu \mathrm{g} / \mathrm{L}$ & -- \\
\hline Alachlor & $\mathrm{X}$ & $\mathrm{X}$ & -- & $.0045 \mu \mathrm{g} / \mathrm{L}$ & -- \\
\hline
\end{tabular}


Table 6. Constituents analyzed in surface-water and streambed-sediment samples collected from selected Johnson County streams, northeastern Kansas, October 2002-June 2004.-Continued

[X, analyzed; --, not analyzed or not applicable; N/A, no laboratory reporting level established; <, less than; E, estimated; $\mu \mathrm{S} / \mathrm{cm}$, microsiemens per centimeter at 25 degrees Celsius; FNU, formazin nephelometric units; $\mathrm{mg} / \mathrm{L}$, milligrams per liter; $\mathrm{mg} / \mathrm{kg}$, milligrams per kilogram; $\mu \mathrm{g} / \mathrm{L}$, micrograms per liter; $\mu \mathrm{g} / \mathrm{kg}$, micrograms per kilogram; col/100 mL, colonies per 100 milliliters]

\begin{tabular}{|c|c|c|c|c|c|}
\hline Constituent & $\begin{array}{l}\text { Base-flow } \\
\text { samples }\end{array}$ & $\begin{array}{l}\text { Stormflow } \\
\text { samples }\end{array}$ & $\begin{array}{l}\text { Streambed- } \\
\text { sediment } \\
\text { sample }\end{array}$ & $\begin{array}{c}\text { Stream-water } \\
\text { laboratory reporting } \\
\text { level }\end{array}$ & $\begin{array}{c}\text { Streambed- } \\
\text { sediment } \\
\text { laboratory } \\
\text { reporting level }\end{array}$ \\
\hline \multicolumn{6}{|c|}{ Pesticide compounds (filtered water analysis)—Continued } \\
\hline Aldrin & -- & -- & $\mathrm{X}$ & -- & $1-3 \mu \mathrm{g} / \mathrm{kg}$ \\
\hline alpha-Endosulfan & -- & -- & $\mathrm{X}$ & -- & $1-3 \mu \mathrm{g} / \mathrm{kg}$ \\
\hline alpha-HCH & $\mathrm{X}$ & -- & $\mathrm{X}$ & -- & $1-3 \mu \mathrm{g} / \mathrm{kg}$ \\
\hline Atrazine & $\mathrm{X}$ & $\mathrm{X}$ & $\mathrm{X}$ & $0.007 \mu \mathrm{g} / \mathrm{L}$ & $100 \mu \mathrm{g} / \mathrm{kg}$ \\
\hline Azinphos-methyl & $\mathrm{X}$ & $\mathrm{X}$ & -- & $.05 \mu \mathrm{g} / \mathrm{L}$ & -- \\
\hline Azinphos-methyl-oxon & $\mathrm{X}$ & $\mathrm{X}$ & -- & $.016 \mu \mathrm{g} / \mathrm{L}$ & -- \\
\hline Benfluralin & $\mathrm{X}$ & $X$ & -- & $.01 \mu \mathrm{g} / \mathrm{L}$ & -- \\
\hline beta-HCH & -- & -- & $X$ & -- & $1-3 \mu \mathrm{g} / \mathrm{kg}$ \\
\hline Butylate & $X^{1}$ & -- & -- & $.02 \mu \mathrm{g} / \mathrm{L}$ & -- \\
\hline Carbaryl & $X$ & $X$ & -- & $.041 \mu \mathrm{g} / \mathrm{L}$ & -- \\
\hline Carbofuran & $X^{1}$ & -- & -- & $.02 \mu \mathrm{g} / \mathrm{L}$ & -- \\
\hline Chloroneb & -- & -- & $\mathrm{X}$ & -- & $5-15 \mu \mathrm{g} / \mathrm{kg}$ \\
\hline Chlorpyrifos & $X$ & $\mathrm{X}$ & $X$ & $.005 \mathrm{ug} / \mathrm{L}$ & -- \\
\hline Chlorpyrofos, oxygen analog & $X$ & $\mathrm{X}$ & -- & $.056 \mu \mathrm{g} / \mathrm{L}$ & $<50 \mu \mathrm{g} / \mathrm{kg}$ \\
\hline cis-Chlordane & -- & -- & $\mathrm{X}$ & -- & $1-3 \mu \mathrm{g} / \mathrm{kg}$ \\
\hline cis-Nonachlor & -- & -- & $\mathrm{X}$ & -- & $1-3 \mu \mathrm{g} / \mathrm{kg}$ \\
\hline cis-Permethrin & $\mathrm{X}$ & $\mathrm{X}$ & $\mathrm{X}$ & $.006 \mu \mathrm{g} / \mathrm{L}$ & $5-15 \mu \mathrm{g} / \mathrm{kg}$ \\
\hline Cyanazine & $X^{1}$ & -- & -- & $.02 \mu \mathrm{g} / \mathrm{L}$ & -- \\
\hline Cyfluthrin & $\mathrm{X}$ & $\mathrm{X}$ & -- & $.008 \mu \mathrm{g} / \mathrm{L}$ & -- \\
\hline Cypermethrin & $X$ & $\mathrm{X}$ & -- & $.0086 \mu \mathrm{g} / \mathrm{L}$ & -- \\
\hline Dacthal & $\mathrm{X}$ & $\mathrm{X}$ & -- & $.003 \mu \mathrm{g} / \mathrm{L}$ & -- \\
\hline DCPA & $\mathrm{X}$ & $\mathrm{X}$ & $\mathrm{X}$ & -- & $5-15 \mu \mathrm{g} / \mathrm{kg}$ \\
\hline Deethylatrazine & $X^{1}$ & -- & -- & $.006 \mu \mathrm{g} / \mathrm{L}$ & -- \\
\hline Desulfinylfipronil & $\mathrm{X}$ & $\mathrm{X}$ & -- & $.004 \mu \mathrm{g} / \mathrm{L}$ & -- \\
\hline Desulfinylfipronil amide & $\mathrm{X}$ & $\mathrm{X}$ & -- & $.009 \mu \mathrm{g} / \mathrm{L}$ & -- \\
\hline Diazinon & $\mathrm{X}$ & $\mathrm{X}$ & $X$ & $.005 \mu \mathrm{g} / \mathrm{L}$ & $<50 \mu \mathrm{g} / \mathrm{kg}$ \\
\hline Diazinon, oxygen analog & $\mathrm{X}$ & $\mathrm{X}$ & -- & .04 (dimensionless) & -- \\
\hline Dichlorvos & $X$ & $\mathrm{X}$ & -- & $.011 \mu \mathrm{g} / \mathrm{L}$ & -- \\
\hline Dicrotophos & $X$ & $\mathrm{X}$ & -- & $.084 \mu \mathrm{g} / \mathrm{L}$ & -- \\
\hline Dieldrin & $X$ & $X$ & $\mathrm{X}$ & $.0048 \mu \mathrm{g} / \mathrm{L}$ & $1-3 \mu \mathrm{g} / \mathrm{kg}$ \\
\hline Dimethoate & $\mathrm{X}$ & $\mathrm{X}$ & -- & $.0061 \mu \mathrm{g} / \mathrm{L}$ & -- \\
\hline Disulfoton & $X^{1}$ & -- & -- & $.02 \mu \mathrm{g} / \mathrm{L}$ & -- \\
\hline Endrin & -- & -- & $X$ & -- & $2-6 \mu \mathrm{g} / \mathrm{kg}$ \\
\hline EPTC & $X^{1}$ & -- & -- & $.002 \mu \mathrm{g} / \mathrm{L}$ & -- \\
\hline
\end{tabular}


Table 6. Constituents analyzed in surface-water and streambed-sediment samples collected from selected Johnson County streams, northeastern Kansas, October 2002-June 2004.-Continued

[X, analyzed; --, not analyzed or not applicable; N/A, no laboratory reporting level established; <, less than; E, estimated; $\mu$ S/cm, microsiemens per centimeter at 25 degrees Celsius; FNU, formazin nephelometric units; mg/L, milligrams per liter; mg/kg, milligrams per kilogram; $\mu \mathrm{g} / \mathrm{L}$, micrograms per liter; $\mu \mathrm{g} / \mathrm{kg}$, micrograms per kilogram; col/100 mL, colonies per 100 milliliters]

\begin{tabular}{|c|c|c|c|c|c|}
\hline Constituent & $\begin{array}{c}\text { Base-flow } \\
\text { samples }\end{array}$ & $\begin{array}{l}\text { Stormflow } \\
\text { samples }\end{array}$ & $\begin{array}{l}\text { Streambed- } \\
\text { sediment } \\
\text { sample }\end{array}$ & $\begin{array}{c}\text { Stream-water } \\
\text { laboratory reporting } \\
\text { level }\end{array}$ & $\begin{array}{l}\text { Streambed- } \\
\text { sediment } \\
\text { laboratory } \\
\text { reporting level }\end{array}$ \\
\hline \multicolumn{6}{|c|}{ Pesticide compounds (filtered water analysis) —Continued } \\
\hline Ethafluralin & $X^{1}$ & -- & -- & $0.009 \mu \mathrm{g} / \mathrm{L}$ & -- \\
\hline Ethion & $\mathrm{X}$ & $X$ & -- & $.004 \mu \mathrm{g} / \mathrm{L}$ & -- \\
\hline Ethion monoxon & $\mathrm{X}$ & $\mathrm{X}$ & -- & $.033 \mu \mathrm{g} / \mathrm{L}$ & -- \\
\hline Ethoprop & $X^{1}$ & -- & -- & $.005 \mu \mathrm{g} / \mathrm{L}$ & -- \\
\hline Fenamiphos & $\mathrm{X}$ & $\mathrm{X}$ & -- & $.029 \mu \mathrm{g} / \mathrm{L}$ & -- \\
\hline Fenamiphos sulfone & $\mathrm{X}$ & $\mathrm{X}$ & -- & $.0077 \mu \mathrm{g} / \mathrm{L}$ & -- \\
\hline Fipronil & $\mathrm{X}$ & $\mathrm{X}$ & -- & $.007 \mu \mathrm{g} / \mathrm{L}$ & -- \\
\hline Fipronil sulfide & $\mathrm{X}$ & $\mathrm{X}$ & -- & $.005 \mu \mathrm{g} / \mathrm{L}$ & -- \\
\hline Fipronil sulfone & $\mathrm{X}$ & $\mathrm{X}$ & -- & $.005 \mu \mathrm{g} / \mathrm{L}$ & -- \\
\hline Fonofos & $\mathrm{X}$ & $\mathrm{X}$ & -- & $.0027 \mu \mathrm{g} / \mathrm{L}$ & -- \\
\hline Fonofos, oxygen analog & $\mathrm{X}$ & $\mathrm{X}$ & -- & $.0021 \mu \mathrm{g} / \mathrm{L}$ & -- \\
\hline Heptachlor epoxide & -- & -- & $\mathrm{X}$ & -- & $1-3 \mu \mathrm{g} / \mathrm{kg}$ \\
\hline Heptachlor & -- & -- & $\mathrm{X}$ & -- & $1-3 \mu \mathrm{g} / \mathrm{kg}$ \\
\hline Hexachlorobenzene & -- & -- & $X$ & -- & $1-3 \mu \mathrm{g} / \mathrm{kg}$ \\
\hline Hexazinone & $\mathrm{X}$ & $X$ & -- & $.013 \mu \mathrm{g} / \mathrm{L}$ & -- \\
\hline Iprodione & $\mathrm{X}$ & $\mathrm{X}$ & -- & $1.4 \mu \mathrm{g} / \mathrm{L}$ & -- \\
\hline Isodrin & -- & -- & $\mathrm{X}$ & -- & $1-3 \mu \mathrm{g} / \mathrm{kg}$ \\
\hline Isofenphos & $\mathrm{X}$ & $\mathrm{X}$ & -- & $.0034 \mu \mathrm{g} / \mathrm{L}$ & -- \\
\hline Lindane & $\mathrm{X}$ & -- & $\mathrm{X}$ & $.004 \mu \mathrm{g} / \mathrm{L}$ & $1-3 \mu \mathrm{g} / \mathrm{kg}$ \\
\hline Linuuron & $X^{1}$ & -- & -- & $.035 \mu \mathrm{g} / \mathrm{L}$ & -- \\
\hline Malaoxon & $\mathrm{X}$ & $\mathrm{X}$ & -- & $.008 \mu \mathrm{g} / \mathrm{L}$ & -- \\
\hline Malathion & $X$ & $\mathrm{X}$ & -- & $.027 \mu \mathrm{g} / \mathrm{L}$ & -- \\
\hline Metalaxyl & $\mathrm{X}$ & $\mathrm{X}$ & $X$ & $.05 \mu \mathrm{g} / \mathrm{L}$ & $<100 \mu \mathrm{g} / \mathrm{kg}$ \\
\hline Metolachlor & $X$ & $\mathrm{X}$ & $\mathrm{X}$ & $.013 \mu \mathrm{g} / \mathrm{L}$ & $<50 \mu \mathrm{g} / \mathrm{kg}$ \\
\hline Metribuzin & $\mathrm{X}$ & $\mathrm{X}$ & -- & $.006 \mu \mathrm{g} / \mathrm{L}$ & -- \\
\hline Myclobutanil & $\mathrm{X}$ & $\mathrm{X}$ & -- & $.008 \mu \mathrm{g} / \mathrm{L}$ & -- \\
\hline Mirex & -- & -- & $X$ & -- & $1-3 \mu \mathrm{g} / \mathrm{kg}$ \\
\hline Molinate & $X^{1}$ & -- & -- & $.002 \mu \mathrm{g} / \mathrm{L}$ & -- \\
\hline Napropamide & $X^{1}$ & -- & -- & $.007 \mu \mathrm{g} / \mathrm{L}$ & -- \\
\hline$o, p^{\prime}-\mathrm{DDD}$ & -- & -- & $\mathrm{X}$ & -- & $1-3 \mu \mathrm{g} / \mathrm{kg}$ \\
\hline o,p'-DDE & -- & -- & $X$ & -- & $1-3 \mu \mathrm{g} / \mathrm{kg}$ \\
\hline o,p'-DDT & -- & -- & $X$ & -- & $2-6 \mu \mathrm{g} / \mathrm{kg}$ \\
\hline o,p'-Methoxychlor & -- & -- & $\mathrm{X}$ & -- & $5-15 \mu \mathrm{g} / \mathrm{kg}$ \\
\hline Oxychlordane & -- & -- & $X$ & -- & $1-3 \mu \mathrm{g} / \mathrm{kg}$ \\
\hline
\end{tabular}


Table 6. Constituents analyzed in surface-water and streambed-sediment samples collected from selected Johnson County streams, northeastern Kansas, October 2002-June 2004.-Continued

[X, analyzed; --, not analyzed or not applicable; N/A, no laboratory reporting level established; <, less than; E, estimated; $\mu \mathrm{S} / \mathrm{cm}$, microsiemens per centimeter at 25 degrees Celsius; FNU, formazin nephelometric units; $\mathrm{mg} / \mathrm{L}$, milligrams per liter; $\mathrm{mg} / \mathrm{kg}$, milligrams per kilogram; $\mu \mathrm{g} / \mathrm{L}$, micrograms per liter; $\mu \mathrm{g} / \mathrm{kg}$, micrograms per kilogram; col/100 mL, colonies per 100 milliliters]

\begin{tabular}{|c|c|c|c|c|c|}
\hline Constituent & $\begin{array}{l}\text { Base-flow } \\
\text { samples }\end{array}$ & $\begin{array}{l}\text { Stormflow } \\
\text { samples }\end{array}$ & $\begin{array}{l}\text { Streambed- } \\
\text { sediment } \\
\text { sample }\end{array}$ & $\begin{array}{c}\text { Stream-water } \\
\text { laboratory reporting } \\
\text { level }\end{array}$ & $\begin{array}{c}\text { Streambed- } \\
\text { sediment } \\
\text { laboratory } \\
\text { reporting level }\end{array}$ \\
\hline \multicolumn{6}{|c|}{ Pesticide compounds (filtered water analysis) —Continued } \\
\hline p,p'-DDD & -- & -- & $\mathrm{X}$ & -- & $1-3 \mu \mathrm{g} / \mathrm{kg}$ \\
\hline $\mathrm{p}, \mathrm{p}$ '-DDE & $\mathrm{X}^{1}$ & -- & $\mathrm{X}$ & $0.003 \mu \mathrm{g} / \mathrm{L}$ & $1-3 \mu \mathrm{g} / \mathrm{kg}$ \\
\hline p,p'-DDT & -- & -- & $\mathrm{X}$ & -- & $2-6 \mu \mathrm{g} / \mathrm{kg}$ \\
\hline p,p'-Methoxychlor & -- & -- & $\mathrm{X}$ & -- & $5-15 \mu \mathrm{g} / \mathrm{kg}$ \\
\hline Paraoxon-methyl & $\mathrm{X}$ & $\mathrm{X}$ & -- & $.029 \mu \mathrm{g} / \mathrm{L}$ & -- \\
\hline Parathion & $X^{1}$ & -- & -- & $.01 \mu \mathrm{g} / \mathrm{L}$ & -- \\
\hline Parathion-methyl & $\mathrm{X}$ & $\mathrm{X}$ & -- & $.006 \mu \mathrm{g} / \mathrm{L}$ & -- \\
\hline Pebulate & $\mathrm{X}^{1}$ & -- & -- & $.004 \mu \mathrm{g} / \mathrm{L}$ & -- \\
\hline Pendimethalin & $\mathrm{X}$ & $\mathrm{X}$ & -- & $.022 \mu \mathrm{g} / \mathrm{L}$ & -- \\
\hline Pentachloroanisole & -- & -- & $X$ & -- & $1-3 \mu \mathrm{g} / \mathrm{kg}$ \\
\hline Phorate & $\mathrm{X}$ & $\mathrm{X}$ & -- & $.011 \mu \mathrm{g} / \mathrm{L}$ & -- \\
\hline Phorate oxon & $X$ & $\mathrm{X}$ & -- & $.097 \mu \mathrm{g} / \mathrm{L}$ & -- \\
\hline Phosmet & $X$ & $\mathrm{X}$ & -- & $.0079 \mu \mathrm{g} / \mathrm{L}$ & -- \\
\hline Phosmet oxon & $X$ & $\mathrm{X}$ & -- & $.055 \mu \mathrm{g} / \mathrm{L}$ & -- \\
\hline Polychlorinated biphenyls (PCBs) & -- & -- & $\mathrm{X}$ & -- & $50-150 \mu \mathrm{g} / \mathrm{kg}$ \\
\hline Prometon & $\mathrm{X}$ & $\mathrm{X}$ & $\mathrm{X}$ & $.015 \mu \mathrm{g} / \mathrm{L}$ & $<50 \mu \mathrm{g} / \mathrm{kg}$ \\
\hline Prometryn & $\mathrm{X}$ & $\mathrm{X}$ & -- & $.0054 \mu \mathrm{g} / \mathrm{L}$ & -- \\
\hline Pronamide & $\mathrm{X}^{1}$ & -- & -- & $.004 \mu \mathrm{g} / \mathrm{L}$ & -- \\
\hline Propachlor & $\mathrm{X}^{1}$ & -- & -- & $.01 \mu \mathrm{g} / \mathrm{L}$ & -- \\
\hline Propanil & $\mathrm{X}^{1}$ & -- & -- & $.011 \mu \mathrm{g} / \mathrm{L}$ & -- \\
\hline Propargite & $X^{1}$ & -- & -- & $.02 \mu \mathrm{g} / \mathrm{L}$ & -- \\
\hline Propyzamide & $\mathrm{X}$ & $\mathrm{X}$ & -- & $.0041 \mu \mathrm{g} / \mathrm{L}$ & -- \\
\hline Simazine & $\mathrm{X}$ & $\mathrm{X}$ & -- & $.005 \mu \mathrm{g} / \mathrm{L}$ & -- \\
\hline Tebuthiuron & $\mathrm{X}$ & $\mathrm{X}$ & -- & $.016 \mu \mathrm{g} / \mathrm{L}$ & -- \\
\hline Terbacil & $\mathrm{X}^{1}$ & -- & -- & $.034 \mu \mathrm{g} / \mathrm{L}$ & -- \\
\hline Terbufos & $\mathrm{X}$ & $\mathrm{X}$ & -- & $.017 \mu \mathrm{g} / \mathrm{L}$ & -- \\
\hline Terbufos-oxygen-analogue sulfone & $\mathrm{X}$ & $\mathrm{X}$ & -- & $.067 \mu \mathrm{g} / \mathrm{L}$ & -- \\
\hline Terbuthylazine & $X$ & $\mathrm{X}$ & -- & $.010 \mu \mathrm{g} / \mathrm{L}$ & -- \\
\hline Thiobencarb & $X^{1}$ & -- & -- & $.005 \mu \mathrm{g} / \mathrm{L}$ & -- \\
\hline Toxaphene & -- & -- & $\mathrm{X}$ & -- & $200-600 \mu \mathrm{g} / \mathrm{kg}$ \\
\hline trans-Chlordane & -- & -- & $\mathrm{X}$ & -- & $1-3 \mu \mathrm{g} / \mathrm{kg}$ \\
\hline trans-Nonachlor & -- & -- & $\mathrm{X}$ & -- & $1-3 \mu \mathrm{g} / \mathrm{kg}$ \\
\hline trans-Permethrin & -- & -- & $\mathrm{X}$ & -- & $5-15 \mu \mathrm{g} / \mathrm{kg}$ \\
\hline Triallate & $\mathrm{X}^{1}$ & -- & -- & $.002 \mu \mathrm{g} / \mathrm{L}$ & -- \\
\hline
\end{tabular}


Table 6. Constituents analyzed in surface-water and streambed-sediment samples collected from selected Johnson County streams, northeastern Kansas, October 2002-June 2004.-Continued

[X, analyzed; --, not analyzed or not applicable; N/A, no laboratory reporting level established; <, less than; E, estimated; $\mu$ S/cm, microsiemens per centimeter at 25 degrees Celsius; FNU, formazin nephelometric units; mg/L, milligrams per liter; mg/kg, milligrams per kilogram; $\mu \mathrm{g} / \mathrm{L}$, micrograms per liter; $\mu \mathrm{g} / \mathrm{kg}$, micrograms per kilogram; col/100 mL, colonies per 100 milliliters]

\begin{tabular}{|c|c|c|c|c|c|}
\hline Constituent & $\begin{array}{l}\text { Base-flow } \\
\text { samples }\end{array}$ & $\begin{array}{l}\text { Stormflow } \\
\text { samples }\end{array}$ & $\begin{array}{l}\text { Streambed- } \\
\text { sediment } \\
\text { sample }\end{array}$ & $\begin{array}{c}\text { Stream-water } \\
\text { laboratory reporting } \\
\text { level }\end{array}$ & $\begin{array}{l}\text { Streambed- } \\
\text { sediment } \\
\text { laboratory } \\
\text { reporting level }\end{array}$ \\
\hline \multicolumn{6}{|c|}{ Pesticide compounds (filtered water analysis) — Continued } \\
\hline Tribromomethane & $\mathrm{X}$ & $\mathrm{X}$ & -- & $0.5 \mu \mathrm{g} / \mathrm{L}$ & -- \\
\hline Trifluralin & $\mathrm{X}$ & $\mathrm{X}$ & -- & $.009 \mu \mathrm{g} / \mathrm{L}$ & -- \\
\hline \multicolumn{6}{|c|}{ Wastewater compounds } \\
\hline 1,4 Dichlorobenzene & $\mathrm{X}$ & $\mathrm{X}$ & $\mathrm{X}$ & $.5 \mu \mathrm{g} / \mathrm{L}$ & $<50 \mu \mathrm{g} / \mathrm{kg}$ \\
\hline 1-Methylnaphthalene & $X$ & $X$ & $X$ & $.5 \mu \mathrm{g} / \mathrm{L}$ & $<50 \mu \mathrm{g} / \mathrm{kg}$ \\
\hline 17-alpha-ethynyl-esterdiol & -- & -- & $X$ & -- & $<250 \mu \mathrm{g} / \mathrm{kg}$ \\
\hline 17-beta-estradiol & -- & -- & $X$ & -- & $<250 \mu \mathrm{g} / \mathrm{kg}$ \\
\hline 2,6-Dimethylnaphthalene & $\mathrm{X}$ & $\mathrm{X}$ & $\mathrm{X}$ & $.5 \mu \mathrm{g} / \mathrm{L}$ & $<50 \mu \mathrm{g} / \mathrm{kg}$ \\
\hline 2-Methylnaphthalene & $\mathrm{X}$ & $\mathrm{X}$ & $\mathrm{X}$ & $.5 \mu \mathrm{g} / \mathrm{L}$ & $<50 \mu \mathrm{g} / \mathrm{kg}$ \\
\hline 3,4-dichlorophenyl isocyanate & -- & -- & $\mathrm{X}$ & -- & $<100 \mu \mathrm{g} / \mathrm{kg}$ \\
\hline 3-Beta-coprostanol & $X$ & $\mathrm{X}$ & $X$ & $2.0 \mu \mathrm{g} / \mathrm{L}$ & $<200 \mu \mathrm{g} / \mathrm{kg}$ \\
\hline 3-Methyl-1(H)-indole (Skatol) & $\mathrm{X}$ & $\mathrm{X}$ & $\mathrm{X}$ & $1.0 \mu \mathrm{g} / \mathrm{L}$ & $<50 \mu \mathrm{g} / \mathrm{kg}$ \\
\hline 3-Tert-Butyl-4-hydroxy anisole (BHA) & $\mathrm{X}$ & $\mathrm{X}$ & -- & $5.0 \mu \mathrm{g} / \mathrm{L}$ & -- \\
\hline 4-Cumylphenol & $\mathrm{X}$ & $\mathrm{X}$ & $\mathrm{X}$ & $1.0 \mu \mathrm{g} / \mathrm{L}$ & $<50 \mu \mathrm{g} / \mathrm{kg}$ \\
\hline 4-Nonylphenol & $\mathrm{X}$ & $\mathrm{X}$ & $\mathrm{X}$ & $5.0 \mu \mathrm{g} / \mathrm{L}$ & $<500 \mu \mathrm{g} / \mathrm{kg}$ \\
\hline 4-Octylphenol & $\mathrm{X}$ & $\mathrm{X}$ & $\mathrm{X}$ & $1.0 \mu \mathrm{g} / \mathrm{L}$ & $<50 \mu \mathrm{g} / \mathrm{kg}$ \\
\hline 4-Tert-octylphenol & $\mathrm{X}$ & $\mathrm{X}$ & $\mathrm{X}$ & $1.0 \mu \mathrm{g} / \mathrm{L}$ & $<50 \mu \mathrm{g} / \mathrm{kg}$ \\
\hline 5-Methyl-1H-benzotriazole & $\mathrm{X}$ & $\mathrm{X}$ & -- & $2.0 \mu \mathrm{g} / \mathrm{L}$ & -- \\
\hline 9,10 -Anthraquinone & $\mathrm{X}$ & $\mathrm{X}$ & $\mathrm{X}$ & $.5 \mu \mathrm{g} / \mathrm{L}$ & $<50 \mu \mathrm{g} / \mathrm{kg}$ \\
\hline Acetophenone & $\mathrm{X}$ & $\mathrm{X}$ & $\mathrm{X}$ & $.5 \mu \mathrm{g} / \mathrm{L}$ & $<50 \mu \mathrm{g} / \mathrm{kg}$ \\
\hline $\begin{array}{l}\text { Acetyl-hexamethyl-tetrahydro-naphthalene } \\
\text { (AHTN) }\end{array}$ & $\mathrm{X}$ & $\mathrm{X}$ & $\mathrm{X}$ & $.5 \mu \mathrm{g} / \mathrm{L}$ & $<50 \mu \mathrm{g} / \mathrm{kg}$ \\
\hline Anthracene & $\mathrm{X}$ & $\mathrm{X}$ & $\mathrm{X}$ & $.5 \mu \mathrm{g} / \mathrm{L}$ & $<50 \mu \mathrm{g} / \mathrm{kg}$ \\
\hline Benzo A Pyrene & $\mathrm{X}$ & $\mathrm{X}$ & $\mathrm{X}$ & $.5 \mu \mathrm{g} / \mathrm{L}$ & $<50 \mu \mathrm{g} / \mathrm{kg}$ \\
\hline Benzophenone & $\mathrm{X}$ & $\mathrm{X}$ & $X$ & $.5 \mu \mathrm{g} / \mathrm{L}$ & $<50 \mu \mathrm{g} / \mathrm{kg}$ \\
\hline beta-Sitosterol & $\mathrm{X}$ & $\mathrm{X}$ & $\mathrm{X}$ & $2.0 \mu \mathrm{g} / \mathrm{L}$ & $<500 \mu \mathrm{g} / \mathrm{kg}$ \\
\hline beta-Stigmastanol & $X$ & $X$ & $X$ & $2.0 \mu \mathrm{g} / \mathrm{L}$ & $<500 \mu \mathrm{g} / \mathrm{kg}$ \\
\hline Bisphenol A & $X$ & $X$ & $X$ & $1.0 \mu \mathrm{g} / \mathrm{L}$ & $<100 \mu \mathrm{g} / \mathrm{kg}$ \\
\hline Bromacil & $\mathrm{X}$ & $\mathrm{X}$ & $\mathrm{X}$ & $.5 \mu \mathrm{g} / \mathrm{L}$ & $<100 \mu \mathrm{g} / \mathrm{kg}$ \\
\hline Bromoform & -- & -- & $\mathrm{X}$ & -- & $<50 \mu \mathrm{g} / \mathrm{kg}$ \\
\hline Caffeine & $\mathrm{X}$ & $\mathrm{X}$ & -- & $.5 \mu \mathrm{g} / \mathrm{L}$ & -- \\
\hline Camphor & $\mathrm{X}$ & $\mathrm{X}$ & $\mathrm{X}$ & $.5 \mu \mathrm{g} / \mathrm{L}$ & $<50 \mu \mathrm{g} / \mathrm{kg}$ \\
\hline Carbazole & $\mathrm{X}$ & $\mathrm{X}$ & $\mathrm{X}$ & $.5 \mu \mathrm{g} / \mathrm{L}$ & $<50 \mu \mathrm{g} / \mathrm{kg}$ \\
\hline Cholesterol & $\mathrm{X}$ & $\mathrm{X}$ & $\mathrm{X}$ & $2.0 \mu \mathrm{g} / \mathrm{L}$ & $<200 \mu \mathrm{g} / \mathrm{kg}$ \\
\hline Cotinine & $X$ & $\mathrm{X}$ & -- & $1.0 \mu \mathrm{g} / \mathrm{L}$ & -- \\
\hline
\end{tabular}


Table 6. Constituents analyzed in surface-water and streambed-sediment samples collected from selected Johnson County streams, northeastern Kansas, October 2002-June 2004.-Continued

[X, analyzed; --, not analyzed or not applicable; N/A, no laboratory reporting level established; <, less than; E, estimated; $\mu \mathrm{S} / \mathrm{cm}$, microsiemens per centimeter at 25 degrees Celsius; FNU, formazin nephelometric units; $\mathrm{mg} / \mathrm{L}$, milligrams per liter; $\mathrm{mg} / \mathrm{kg}$, milligrams per kilogram; $\mu \mathrm{g} / \mathrm{L}$, micrograms per liter; $\mu \mathrm{g} / \mathrm{kg}$, micrograms per kilogram; col/100 mL, colonies per 100 milliliters]

\begin{tabular}{|c|c|c|c|c|c|}
\hline Constituent & $\begin{array}{l}\text { Base-flow } \\
\text { samples }\end{array}$ & $\begin{array}{l}\text { Stormflow } \\
\text { samples }\end{array}$ & $\begin{array}{l}\text { Streambed- } \\
\text { sediment } \\
\text { sample }\end{array}$ & $\begin{array}{c}\text { Stream-water } \\
\text { laboratory reporting } \\
\text { level }\end{array}$ & $\begin{array}{c}\text { Streambed- } \\
\text { sediment } \\
\text { laboratory } \\
\text { reporting level }\end{array}$ \\
\hline \multicolumn{6}{|c|}{ Wastewater compounds-Continued } \\
\hline N,N-diethyl-meta-toluamide (DEET) & $\mathrm{X}$ & $\mathrm{X}$ & $\mathrm{X}$ & $0.5 \mu \mathrm{g} / \mathrm{L}$ & $<100 \mu \mathrm{g} / \mathrm{kg}$ \\
\hline Diethyl phthalate & -- & -- & $\mathrm{X}$ & -- & E16 \\
\hline Diethylhexyl phthalate & -- & -- & $\mathrm{X}$ & -- & E29 \\
\hline d-Limonene & $\mathrm{X}$ & $\mathrm{X}$ & $\mathrm{X}$ & $.5 \mu \mathrm{g} / \mathrm{L}$ & $<50 \mu \mathrm{g} / \mathrm{kg}$ \\
\hline Equilenin & -- & -- & $\mathrm{X}$ & -- & $<100 \mu \mathrm{g} / \mathrm{kg}$ \\
\hline Estrone & -- & -- & $\mathrm{X}$ & -- & $<250 \mu \mathrm{g} / \mathrm{kg}$ \\
\hline Fluoranthene & $\mathrm{X}$ & $\mathrm{X}$ & $\mathrm{X}$ & $.5 \mu \mathrm{g} / \mathrm{L}$ & $<50 \mu \mathrm{g} / \mathrm{kg}$ \\
\hline $\begin{array}{l}\text { Hexahydro-hexamethyl-cyclopentabenzopyran } \\
\text { (HHCB) }\end{array}$ & $\mathrm{X}$ & $\mathrm{X}$ & $\mathrm{X}$ & $.5 \mu \mathrm{g} / \mathrm{L}$ & $<50 \mu \mathrm{g} / \mathrm{kg}$ \\
\hline Indole & $\mathrm{X}$ & $\mathrm{X}$ & $\mathrm{X}$ & $.5 \mu \mathrm{g} / \mathrm{L}$ & $<50 \mu \mathrm{g} / \mathrm{kg}$ \\
\hline Isoborneol & $\mathrm{X}$ & $\mathrm{X}$ & $\mathrm{X}$ & $.5 \mu \mathrm{g} / \mathrm{L}$ & $<50 \mu \mathrm{g} / \mathrm{kg}$ \\
\hline Isophorone & $\mathrm{X}$ & $\mathrm{X}$ & $\mathrm{X}$ & $.5 \mu \mathrm{g} / \mathrm{L}$ & $<50 \mu \mathrm{g} / \mathrm{kg}$ \\
\hline Isopropyl benzene (Cumene) & $\mathrm{X}$ & $\mathrm{X}$ & $\mathrm{X}$ & $.5 \mu \mathrm{g} / \mathrm{L}$ & $<50 \mu \mathrm{g} / \mathrm{kg}$ \\
\hline Isoquinonline & $\mathrm{X}$ & $\mathrm{X}$ & $\mathrm{X}$ & $.5 \mu \mathrm{g} / \mathrm{L}$ & $<50 \mu \mathrm{g} / \mathrm{kg}$ \\
\hline Menthol & $\mathrm{X}$ & $\mathrm{X}$ & $\mathrm{X}$ & $.5 \mu \mathrm{g} / \mathrm{L}$ & $<50 \mu \mathrm{g} / \mathrm{kg}$ \\
\hline Methyl salicylate & $\mathrm{X}$ & $\mathrm{X}$ & $\mathrm{X}$ & $.5 \mu \mathrm{g} / \mathrm{L}$ & $<100 \mu \mathrm{g} / \mathrm{kg}$ \\
\hline Naphthalene & $\mathrm{X}$ & $\mathrm{X}$ & $\mathrm{X}$ & $.5 \mu \mathrm{g} / \mathrm{L}$ & $<50 \mu \mathrm{g} / \mathrm{kg}$ \\
\hline Nonylphenol-diethoxylate (NPEO2) & $\mathrm{X}$ & $\mathrm{X}$ & $\mathrm{X}$ & $.5 \mu \mathrm{g} / \mathrm{L}$ & $<500 \mu \mathrm{g} / \mathrm{kg}$ \\
\hline Nonylphenol-ethoxylate (NPEO1) & -- & -- & $\mathrm{X}$ & -- & $<500 \mu \mathrm{g} / \mathrm{kg}$ \\
\hline Octylphenol-diethoxylate & $\mathrm{X}$ & $\mathrm{X}$ & $\mathrm{X}$ & $1.0 \mu \mathrm{g} / \mathrm{L}$ & $<100 \mu \mathrm{g} / \mathrm{kg}$ \\
\hline Octylphenol-ethoxylate & $\mathrm{X}$ & $X$ & $\mathrm{X}$ & $1.0 \mu \mathrm{g} / \mathrm{L}$ & $<100 \mu \mathrm{g} / \mathrm{kg}$ \\
\hline Para-cresol & $\mathrm{X}$ & $\mathrm{X}$ & $\mathrm{X}$ & $1.0 \mu \mathrm{g} / \mathrm{L}$ & $<100 \mu \mathrm{g} / \mathrm{kg}$ \\
\hline Pentachlorophenol & $\mathrm{X}$ & $\mathrm{X}$ & $\mathrm{X}$ & $2.0 \mu \mathrm{g} / \mathrm{L}$ & $<200 \mu \mathrm{g} / \mathrm{kg}$ \\
\hline Phenanthrene & $\mathrm{X}$ & $\mathrm{X}$ & $\mathrm{X}$ & $.5 \mu \mathrm{g} / \mathrm{L}$ & $<50 \mu \mathrm{g} / \mathrm{kg}$ \\
\hline Phenol & $\mathrm{X}$ & $\mathrm{X}$ & $\mathrm{X}$ & $.5 \mu \mathrm{g} / \mathrm{L}$ & $<100 \mu \mathrm{g} / \mathrm{kg}$ \\
\hline Pyrene & $\mathrm{X}$ & $\mathrm{X}$ & $\mathrm{X}$ & $.5 \mu \mathrm{g} / \mathrm{L}$ & $<50 \mu \mathrm{g} / \mathrm{kg}$ \\
\hline Tetrabromodiphenyl ether & -- & -- & $\mathrm{X}$ & -- & $<50 \mu \mathrm{g} / \mathrm{kg}$ \\
\hline Tetrachloroethylene & $\mathrm{X}$ & $\mathrm{X}$ & $\mathrm{X}$ & $.5 \mu \mathrm{g} / \mathrm{L}$ & $<50 \mu \mathrm{g} / \mathrm{kg}$ \\
\hline Tributylphosphate & $\mathrm{X}$ & $\mathrm{X}$ & $\mathrm{X}$ & $.5 \mu \mathrm{g} / \mathrm{L}$ & $<50 \mu \mathrm{g} / \mathrm{kg}$ \\
\hline Triclosan & $\mathrm{X}$ & $\mathrm{X}$ & $\mathrm{X}$ & $1.0 \mu \mathrm{g} / \mathrm{L}$ & $<50 \mu \mathrm{g} / \mathrm{kg}$ \\
\hline Triethyl citrate & $\mathrm{X}$ & $\mathrm{X}$ & -- & $.5 \mu \mathrm{g} / \mathrm{L}$ & -- \\
\hline Triphenyl phosphate & $X$ & $\mathrm{X}$ & $\mathrm{X}$ & $.5 \mu \mathrm{g} / \mathrm{L}$ & $<100 \mu \mathrm{g} / \mathrm{kg}$ \\
\hline Tris(2-butoxyethyl) phosphate & $\mathrm{X}$ & $\mathrm{X}$ & $\mathrm{X}$ & $.5 \mu \mathrm{g} / \mathrm{L}$ & $<100 \mu \mathrm{g} / \mathrm{kg}$ \\
\hline Tris(2-chloroethyl) phosphate (Fyrol CEF) & $\mathrm{X}$ & $X$ & $\mathrm{X}$ & $.5 \mu \mathrm{g} / \mathrm{L}$ & $<100 \mu \mathrm{g} / \mathrm{kg}$ \\
\hline
\end{tabular}


Table 6. Constituents analyzed in surface-water and streambed-sediment samples collected from selected Johnson County streams, northeastern Kansas, October 2002-June 2004.-Continued

[X, analyzed; --, not analyzed or not applicable; N/A, no laboratory reporting level established; <, less than; E, estimated; $\mu \mathrm{S} / \mathrm{cm}$, microsiemens per centimeter at 25 degrees Celsius; FNU, formazin nephelometric units; $\mathrm{mg} / \mathrm{L}$, milligrams per liter; $\mathrm{mg} / \mathrm{kg}$, milligrams per kilogram; $\mu \mathrm{g} / \mathrm{L}$, micrograms per liter; $\mu \mathrm{g} / \mathrm{kg}$, micrograms per kilogram; col/100 mL, colonies per 100 milliliters]

\begin{tabular}{|c|c|c|c|c|c|}
\hline Constituent & $\begin{array}{l}\text { Base-flow } \\
\text { samples }\end{array}$ & $\begin{array}{l}\text { Stormflow } \\
\text { samples }\end{array}$ & $\begin{array}{l}\text { Streambed- } \\
\text { sediment } \\
\text { sample }\end{array}$ & $\begin{array}{c}\text { Stream-water } \\
\text { laboratory reporting } \\
\text { level }\end{array}$ & $\begin{array}{l}\text { Streambed- } \\
\text { sediment } \\
\text { laboratory } \\
\text { reporting level }\end{array}$ \\
\hline \multicolumn{6}{|c|}{ Wastewater compounds-Continued } \\
\hline Tris(dichlorisopropyl) phosphate (Fyrol PCF) & $\mathrm{X}$ & $\mathrm{X}$ & $\mathrm{X}$ & $0.5 \mu \mathrm{g} / \mathrm{L}$ & $<100 \mu \mathrm{g} / \mathrm{kg}$ \\
\hline \multicolumn{6}{|c|}{ Pharmaceutical compounds } \\
\hline 1,7-dimethylxanthine & $\mathrm{X}$ & -- & $\mathrm{X}$ & $.144 \mu \mathrm{g} / \mathrm{L}$ & N/A \\
\hline Acetaminophen & $\mathrm{X}$ & -- & $\mathrm{X}$ & $.036 \mu \mathrm{g} / \mathrm{L}$ & N/A \\
\hline Azithromycin & $\mathrm{X}$ & -- & $\mathrm{X}$ & $.004 \mu \mathrm{g} / \mathrm{L}$ & N/A \\
\hline Caffeine & $\mathrm{X}$ & -- & $\mathrm{X}$ & $.016 \mu \mathrm{g} / \mathrm{L}$ & N/A \\
\hline Carbamazapine & $\mathrm{X}$ & -- & $\mathrm{X}$ & $.011 \mu \mathrm{g} / \mathrm{L}$ & N/A \\
\hline Cimetidine & $\mathrm{X}$ & -- & $\mathrm{X}$ & $.012 \mu \mathrm{g} / \mathrm{L}$ & N/A \\
\hline Codeine & $X$ & -- & $\mathrm{X}$ & $.015 \mu \mathrm{g} / \mathrm{L}$ & N/A \\
\hline Cotinine & $\mathrm{X}$ & -- & $\mathrm{X}$ & $.014 \mu \mathrm{g} / \mathrm{L}$ & N/A \\
\hline Dehydronifedipine & $\mathrm{X}$ & -- & $\mathrm{X}$ & $.015 \mu \mathrm{g} / \mathrm{L}$ & N/A \\
\hline Diltiazem & $X$ & -- & $\mathrm{X}$ & $.016 \mu \mathrm{g} / \mathrm{L}$ & N/A \\
\hline Diphenhydramine & $X$ & -- & $\mathrm{X}$ & $.015 \mu \mathrm{g} / \mathrm{L}$ & N/A \\
\hline Erythromycin & $\mathrm{X}$ & -- & $\mathrm{X}$ & $.009 \mu \mathrm{g} / \mathrm{L}$ & N/A \\
\hline Fluoxetine & $\mathrm{X}$ & -- & $\mathrm{X}$ & $.014 \mu \mathrm{g} / \mathrm{L}$ & N/A \\
\hline Gemfibrozil & $\mathrm{X}$ & -- & $\mathrm{X}$ & $.013 \mu \mathrm{g} / \mathrm{L}$ & N/A \\
\hline Ibuprofen & $\mathrm{X}$ & -- & $\mathrm{X}$ & $.042 \mu \mathrm{g} / \mathrm{L}$ & N/A \\
\hline Miconazole & $\mathrm{X}$ & -- & $\mathrm{X}$ & $.018 \mu \mathrm{g} / \mathrm{L}$ & N/A \\
\hline Ranitidine & $\mathrm{X}$ & -- & $\mathrm{X}$ & $.013 \mu \mathrm{g} / \mathrm{L}$ & N/A \\
\hline Salbutamol & $\mathrm{X}$ & -- & $\mathrm{X}$ & $.023 \mu \mathrm{g} / \mathrm{L}$ & N/A \\
\hline Sulfamethoxazole & $X$ & -- & $\mathrm{X}$ & $.064 \mu \mathrm{g} / \mathrm{L}$ & N/A \\
\hline Thiabendazole & $X$ & -- & $\mathrm{X}$ & $.011 \mu \mathrm{g} / \mathrm{L}$ & N/A \\
\hline Trimethoprim & $\mathrm{X}$ & -- & $\mathrm{X}$ & $.013 \mu \mathrm{g} / \mathrm{L}$ & N/A \\
\hline Warfarin & $\mathrm{X}$ & -- & $\mathrm{X}$ & $.012 \mu \mathrm{g} / \mathrm{L}$ & N/A \\
\hline
\end{tabular}

${ }^{1}$ Indicates compound was only analyzed during the first base-flow synoptic survey, November 4-7, 2002.

temperatures $\left(120\right.$ and $\left.200^{\circ} \mathrm{C}\right)$ at $2,000 \mathrm{lb} / \mathrm{in}^{2}(40$ minutes at each temperature) using a commercially available instrument. This extract was further cleaned by the use of two solid-phase extraction cartridges (polystyrene divinylbenzene and fluorosil) in tandem. Final analysis was by full-scan capillary-column gas chromatography/mass spectrometry (GC/MS) (S. Zaugg,

USGS, written commun., 2003). Pharmaceutical compounds in streambed sediment were determined using methods described in Furlong and others (2005, in press).

\section{Statistical Analyses}

Parametric and nonparametric statistical analyses were conducted to compare relations between selected water-quality constituents and differences between groups of constituents based on land use and streamflow conditions. Simple linear regression is a parametric statistical analysis used to describe relations between water-quality constituents. Regression relations described in this report are based on logarithmically transformed data (to approximate a normal data distribution) and are used only to compare relations between water-quality 
constituents and to determine the significance of those relations (Helsel and Hirsch, 1992).

Nonparametric analyses used in this report include Kendall's tau correlation test and the Mann-Whitney test of independent groups. Nonparametric tests use ranks of data as opposed to real data values and are used if data are not normally distributed and if parametric statistical techniques are skewed by large outliers. Kendall's tau test is used to determine correlation between water-quality constituents and is used in this report to determine whether water-quality constituents correlate to streamflow conditions. The Mann-Whitney test determines whether there is a statistical difference between the medians of two independent groups. This test is used in this report to determine if land use or streamflow conditions were related to significant differences in median concentrations and loads of waterquality constituents. The probability of error ( $p$ value) is used in this report to determine the significance of statistical tests for all statistical methods. A p value of less than 0.05 (95-percent confidence that the statistical test is valid, or that the compared data sets are different) is used in this report to indicate if a statistical test is significant.

\section{Calculation of Loads}

Instantaneous loads are chemical and biological concentrations and densities multiplied by streamflow and an appropriate conversion factor and provide an estimate of the mass of a constituent transported past a given site during a given time. In contrast to concentration data, instantaneous load calculations provide a perspective on the mass transport of water-quality constituents and are useful in estimating the amount of nonpoint and point sources contributing to water-quality contamination. Hourly loads are used in this report because they best represent mass transport conditions over the time it took to collect a water-quality sample.

\section{Quality Assurance and Quality Control}

Complete results of analysis of all stream-water- and streambed-sediment-quality samples are available on the USGS Web site (http://ks.water.usgs.gov/Kansas/studies/qw/joco) and are on file with USGS in Lawrence, Kansas. Quality-assurance and quality-control samples were collected during both streamwater and streambed-sediment sampling.

\section{Surface Water}

Four suspended-sediment concentration samples were collected using both equal-width increment (Wilde and others, 1998) and point sampling methods (automated or dip sampler) to verify consistency of multiple sampling methods. Mean relative percentage differences (RPDs) between the four samples were 16.5 percent. RPD is calculated for a constituent by dividing the absolute value of the difference between the two concentrations by the mean of the two concentrations. Three of the four point samples underrepresented suspended-sediment concentrations compared to equal-width increment samples; the fourth example had no measured differences. Because differences between sampling methods were minimal, all stormflow samples collected (regardless of sampling methodology) were combined for analysis.

One equipment blank sample and one trip blank sample were collected and analyzed for inorganic and organic constituents during the July 2003 synoptic survey. Blank samples were used to determine if, and to what extent, sample collection and processing introduced bias into environmental samples. Equipment blank samples were obtained by passing highly purified water (blank water) through sampling and laboratory equipment used to collect and process environmental samples. Trip blank samples included the additional step of processing the blank water onsite to include any bias associated with environmental conditions. Inorganic and nutrient constituents in blank samples were estimated at concentrations less than laboratory reporting levels.

Pesticide compounds were not detected in blank samples. Nonylphenol-diethoxylate was the only wastewater compound detected in blank samples and was found at concentrations less than laboratory reporting levels. Acetaminophen and caffeine were detected at less than $0.1-\mu \mathrm{g} / \mathrm{L}$ concentrations in blank samples. Analyses of blank samples generally showed that equipment cleaning, sample collection, and processing procedures did not bias the results of environmental samples.

Sequential replicate stream-water samples were collected onsite to determine the variability associated with sample collection, processing procedures, and laboratory analysis. RPDs were calculated between environmental and replicate samples for compounds most frequently detected in Johnson County streams. Six replicate samples were analyzed for inorganic and nutrient compounds, two for indicator bacteria, five for pesticide and wastewater-indicator compounds, and four for pharmaceutical compounds.

Mean RPDs for dissolved solids, nutrients, fecal coliform, and $E$. coli were within 10 percent for nearly all constituents (table 7). However, mean RPDs of 17.0 percent were calculated between ammonia plus total organic nitrogen samples, 27.9 percent in dissolved phosphorus samples, 13.6 percent in total phosphorus samples, and 18.8 percent in enterococci samples. Many of these differences are due to the small concentrations of these constituents in replicate samples causing small differences in concentrations that reflect large RPDs.

Replicate samples were collected at five sampling sites and analyzed for pesticide and wastewater compounds listed in table 6. Of these paired replicate analyses, 97 percent had both reported with a detectable concentration or both reported as not detected. In the remaining 3 percent, one of the paired analyses had a detectable concentration, whereas the other was reported as not detected. However, only one paired analyses (for acetochlor) had a reported concentration larger than the analytical reporting level. In all other cases, the detected concentration 
Table 7. Mean relative percentage differences between environmental and replicate sample pairs with detections of dissolved solids, major ions, nutrients, trace elements, bacteria, and selected pesticide, wastewater, and pharmaceutical compounds in stream-water samples from Johnson County, northeastern Kansas, October 2002-June 2004.

\begin{tabular}{|c|c|c|}
\hline Constituent (number of sample pairs) & $\begin{array}{l}\text { Number of replicate } \\
\text { pairs with detections }\end{array}$ & $\begin{array}{l}\text { Relative percentage } \\
\text { difference (percent) }\end{array}$ \\
\hline Dissolved solids & 6 & 1.3 \\
\hline \multicolumn{3}{|c|}{ Major ions (6) } \\
\hline Calcium & 6 & 2.4 \\
\hline Magnesium & 6 & 2.3 \\
\hline Potassium & 6 & 5.2 \\
\hline Sodium & 6 & 3.0 \\
\hline Alkalinity & 5 & 2.6 \\
\hline Chloride & 6 & .1 \\
\hline Fluoride & 6 & 0 \\
\hline Silica & 6 & 2.2 \\
\hline Sulfate & 6 & 1.2 \\
\hline \multicolumn{3}{|c|}{ Nutrients (6) } \\
\hline Nitrite, as $\mathrm{N}$ & 4 & 6.2 \\
\hline Nitrite plus nitrate, as $\mathrm{N}$ & 6 & 2.4 \\
\hline Ammonia, as $\mathrm{N}$ & 6 & 0 \\
\hline Ammonia plus dissolved organic nitrogen, as $\mathrm{N}$ & 6 & 7.3 \\
\hline Ammonia plus total organic nitrogen, as $\mathrm{N}$ & 6 & 17.0 \\
\hline Total phosphorus & 6 & 13.6 \\
\hline Orthophosphorus, as $\mathrm{P}$ & 5 & 1.5 \\
\hline Dissolved phosphorus & 6 & 27.9 \\
\hline \multicolumn{3}{|c|}{ Trace elements (6) } \\
\hline Iron & 2 & 37.3 \\
\hline Manganese & 6 & .1 \\
\hline \multicolumn{3}{|c|}{ Bacteria (2) } \\
\hline Fecal coliform & 2 & 7.6 \\
\hline Escherichia coli & 2 & 4.7 \\
\hline Enterococci & 2 & 18.8 \\
\hline \multicolumn{3}{|c|}{ Pesticides (5) } \\
\hline 3,4 Dichloroaniline & 4 & 18.5 \\
\hline Atrazine & 5 & 8.9 \\
\hline Diazinon & 5 & 12.3 \\
\hline Metolachlor & 5 & 3.2 \\
\hline Simazine & 2 & 14.4 \\
\hline \multicolumn{3}{|c|}{ Wastewater compounds (5) } \\
\hline Acetyl-hexamethyl-tetrahydro-napthalene (AHTN) & 3 & 7.3 \\
\hline Caffeine & 4 & 2.3 \\
\hline $\mathrm{N}, \mathrm{N}$-diethyl-meta-toluamide (DEET) & 5 & .6 \\
\hline Nonylphenol-diethoxylate (NPEO2) & 4 & 26.7 \\
\hline Phenol & 2 & 11.1 \\
\hline Tris(2-butoxyethyl) phosphate & 3 & 1.9 \\
\hline \multicolumn{3}{|c|}{ Pharmaceutical compounds (4) } \\
\hline Caffeine & 4 & 25.6 \\
\hline Cotinine & 4 & 15.2 \\
\hline
\end{tabular}


was reported as an estimated concentration less than the analytical reporting level. The results of these replicate analyses indicate the reproducibility in sampling procedures and that the precision of analytical methods for determination of pesticides and wastewater compounds is at acceptable levels and does not introduce substantial bias in the reporting of water-quality information.

Mean RPDs were computed for selected pesticide and wastewater compounds detected at the largest concentrations in Johnson County stream-water samples (table 7). RPDs for these compounds were similar to differences reported for dissolved solids, nutrients, and metals. All RPDs were less than 30 percent, indicating that pesticide and wastewater compound methods were able to distinguish quantitative differences between samples.

Four replicate samples were collected during both synoptic surveys and analyzed for pharmaceutical compounds. Of the 88 replicate pairs analyzed, for the 22 pharmaceutical compounds, only one paired analysis had one detection and one nondetection (acetaminophen).

\section{Streambed Sediment}

Sequential replicate streambed-sediment samples were collected at two streambed-sediment sites (three replicate samples for indicator bacteria) for quality-assurance purposes. Replicate samples analyzed for inorganic and nutrient compounds typically were less than 10-percent different, except for samples with small concentrations near laboratory reporting levels (table 8). Combined replicate data from indicator bacteria analyses (fecal coliform, E. coli, and enterococci) had a mean RPD of 53 percent. Few pesticide compounds were detected in streambed-sediment samples; the mean RPD of those detected was 45 percent. Replicate data on streambed sediment generally showed that nutrient and trace element data were reproducible, whereas indicator bacteria and pesticide compounds were less precise.

Because the methods of determination of wastewater compounds in streambed sediment are newly developed and are currently (2005) unpublished, laboratory reagent blank, spike, and surrogate samples were included for these analyses. Two blank samples were analyzed for wastewater compounds. Analyses of three compounds (diethyl phthalate, diethylhexyl phthalate, and phenol) were detected in blank samples. All of these detections were less than laboratory reporting levels. Spike recoveries of wastewater-indicator compounds ranged from 0.8 to 106 percent and had a mean of 74 percent. Recoveries of most compounds were in the range of 50 to 110 percent with the exception of acetophenone, beta-sitosterol, bromoform, isophorone, isoquinoline, methyl salicylate, pentachloroethylene, and tetrachloroethylene, which were all detected at less than 50 percent of the original spike concentration in streambedsediment samples. Low spike recoveries of these compounds indicate that they may be underrepresented in streambedsediment samples. Surrogate recoveries ranged from 5.8 to 113 percent. Inaccurate surrogate recoveries indicate that characteristics of the sample interfered with the isolation, detec- tion, and quantification of the surrogate. Generally surrogate recoveries were low, indicating the potential for underreporting wastewater compounds in streambed-sediment samples.

Of the paired replicate analyses for wastewater compounds in streambed sediment, 92 percent had both reported with a detectable concentration or reported as nondetected. In the remaining 8 percent, only one paired analyses (carbazole) had a reported concentration larger than the analytical reporting level. Few wastewater compounds recorded detections in both streambed-sediment replicate samples; however, many compounds were detected in one replicate pair (table 8). RPDs for wastewater compounds ranged from 8 to 114 percent, illustrating that field and laboratory methods used to collect and analyze these samples had substantial variability. Replicate data on stream-bed sediment generally showed that procedures for determining the presence and (or) absence of wastewater compounds generally were precise. Determination of quantitative differences between samples was less accurate than other analytical methods; wastewater compound methods were likely to underreport wastewater compound occurrence in streambed sediment.

Methods for determining pharmaceutical compounds in streambed sediment are newly developed and currently (2005) unpublished. Laboratory blanks, reagent spikes, and surrogates were determined to validate the analytical methods used in this analysis. Pharmaceutical compounds were not detected in laboratory blank samples, indicating that the analytical method introduced no contamination. Percentage recoveries of spike samples ranged from 0 to 237 percent. Only 2 percent of the spike recoveries were greater than 100 percent, indicating that most pharmaceutical concentrations in streambed sediment likely were underreported. The compounds azithromycin, fluoxetine, ibuprofen, metformin, miconazole, and ranitidine had mean spike recoveries that were less than 10 percent, indicating that these compounds may not have been found in Johnson County streambed sediment due to the inability of analytical methods to extract and recover these compounds. Surrogate recoveries in Johnson County streambed-sediment samples ranged from 0.8 to 21 percent. These low recoveries further indicate the underrepresentation of pharmaceutical compounds in streambed sediment likely due to interference from components of the sediment samples.

Two replicate analyses were collected sequentially for pharmaceutical compounds in streambed sediment. Three pharmaceutical compounds were detected in both an original and a replicate sample; RPDs for these compounds are listed in table 8 . Of the paired replicate analyses for pharmaceutical compounds in streambed-sediment samples, four of the paired analyses had a detectable concentration, whereas the other was reported as not detected. Three of these detections had less than microgram-per-kilogram concentrations; the other (caffeine) had a detection of $6.3 \mu \mathrm{g} / \mathrm{kg}$. The mean RPD of pharmaceutical compounds detected in both replicate samples was 28 percent. Pharmaceutical quality-assurance and control data indicate that interpretation of pharmaceutical data for streambed sediment is limited to distinguishing large-scale differences among sites and compounds. 
Table 8. Mean relative percentage differences between environmental and replicate sample pairs with detections of nutrients, trace elements, bacteria, and pesticide, wastewater, and pharmaceutical compounds in streambed-sediment samples from Johnson County, northeastern Kansas, October 2002-June 2004.

\begin{tabular}{|c|c|c|}
\hline Constituent & $\begin{array}{l}\text { Number of replicate } \\
\text { pairs with detections }\end{array}$ & $\begin{array}{l}\text { Relative percentage } \\
\text { difference (percent) }\end{array}$ \\
\hline \multicolumn{3}{|c|}{ Nutrients } \\
\hline Nitrogen & 2 & 5.8 \\
\hline Phosphorus & 2 & 10 \\
\hline \multicolumn{3}{|c|}{ Trace elements } \\
\hline Arsenic & 2 & 6.6 \\
\hline Lead & 2 & 4.4 \\
\hline Nickel & 2 & .8 \\
\hline Zinc & 2 & 8.1 \\
\hline \multicolumn{3}{|c|}{ Indicator bacteria } \\
\hline Fecal coliform & 2 & 68 \\
\hline Escherichia coli & 2 & 38 \\
\hline Enterococci & 3 & 54 \\
\hline \multicolumn{3}{|c|}{ Pesticide compounds } \\
\hline cis-Chlordane & 1 & 40 \\
\hline Polychlorinated biphenyls (PCBs) & 1 & 33 \\
\hline trans-chlordane & 1 & 40 \\
\hline trans-Nonachlor & 1 & 67 \\
\hline \multicolumn{3}{|c|}{ Wastewater compounds } \\
\hline 9,10-Anthraquinone & 1 & 88 \\
\hline Anthracene & 1 & 114 \\
\hline Benzo(a) pyrene & 1 & 82 \\
\hline beta-Sitosterol & 2 & 56 \\
\hline beta-Stigmastanol & 2 & 81 \\
\hline Carbazole & 1 & 102 \\
\hline Cholesterol & 2 & 37 \\
\hline Diethylhexyl phthalate & 1 & 73 \\
\hline Fluoranthrene & 1 & 83 \\
\hline Indole & 2 & 56 \\
\hline Napthalene & 1 & 61 \\
\hline para-Cresol & 2 & 39 \\
\hline Phenanthrene & 2 & 44 \\
\hline Phenol & 1 & 22 \\
\hline Pyrene & 1 & 81 \\
\hline Skatol & 1 & 8.0 \\
\hline \multicolumn{3}{|c|}{ Pharmaceutical compounds } \\
\hline Caffeine & 1 & .2 \\
\hline Cotinine & 1 & 35 \\
\hline Diphenhydramine & 1 & 50 \\
\hline
\end{tabular}




\section{Hydrologic Conditions}

\section{Precipitation}

Synoptic base-flow samples in November 2002 and July 2003 generally were collected during and after periods of little or no rainfall, whereas stormflow samples were collected during and after periods of increased precipitation, as exemplified at site MI7 (Mill Creek at Johnson Drive, fig. 1) (fig. 5). However, site MI7 shows an exception to this generalization. A small amount of rainfall on November 5, 2002, led to a slight increase in discharge at the farthest downstream sites where samples were collected on this date (which also included Blue River samples) (fig. 6).
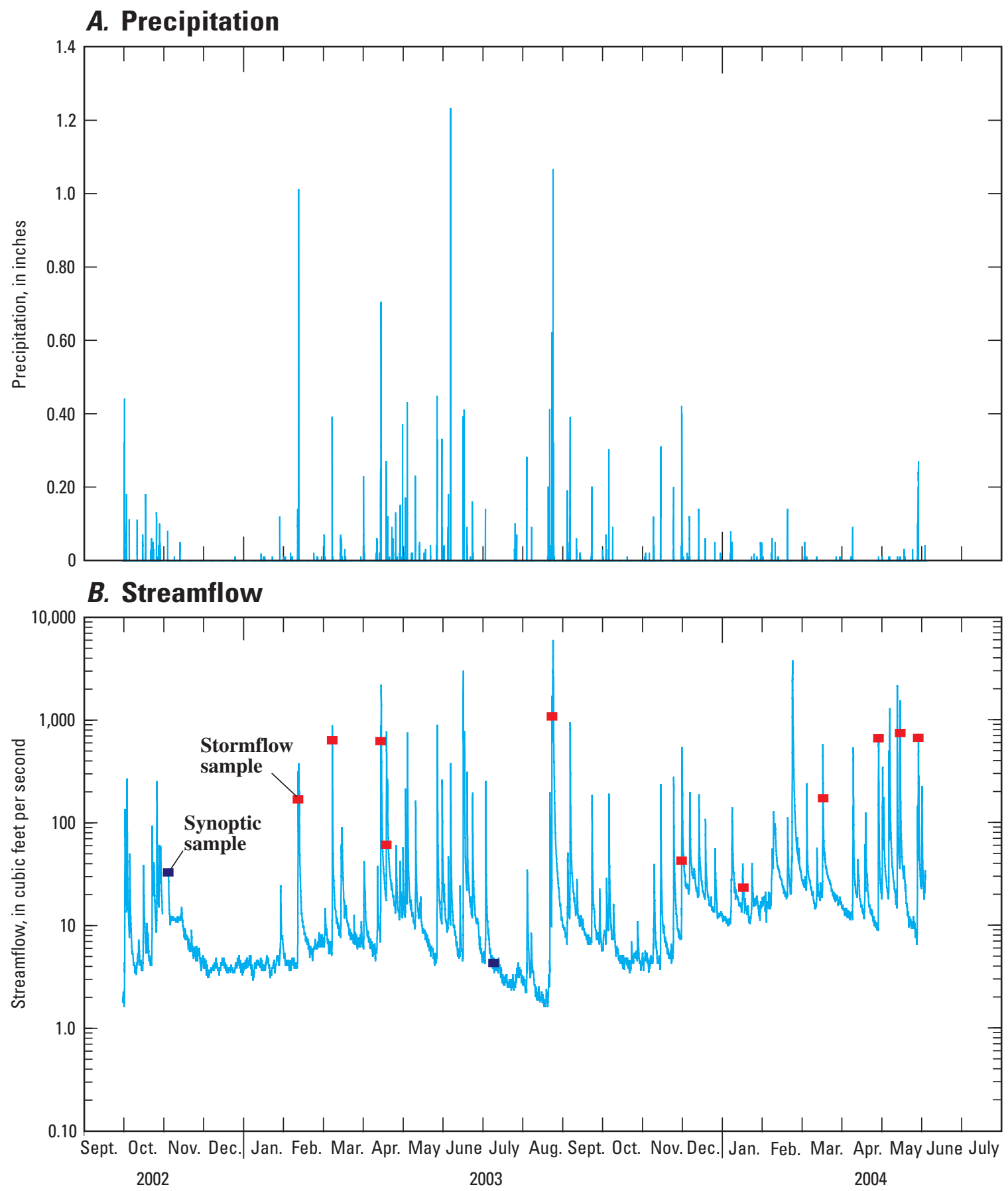

Figure 5. Comparison of precipitation and streamflow at Mill Creek at Johnson Drive (site MI7, fig. 1) during stream-water sample collection, October 2002-June 2004. 


\section{A. Big Bull Creek watershed}

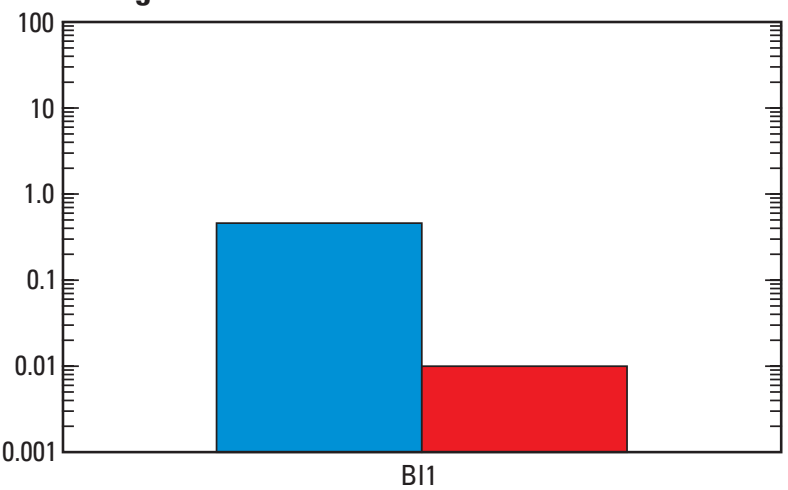

\section{Brush Creek watershed}

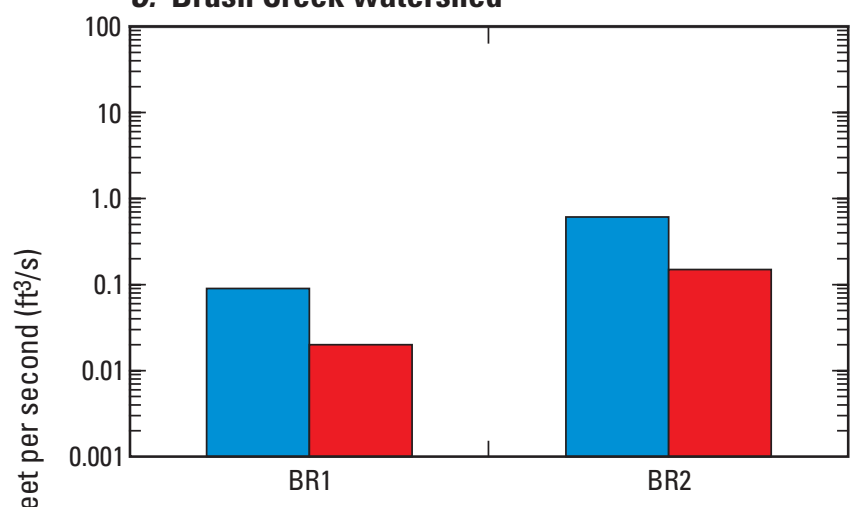

E. Dykes Branch watershed

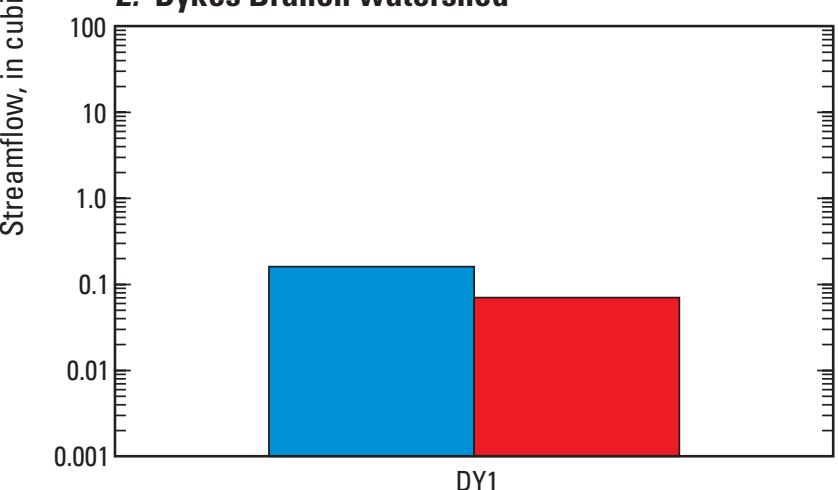

G. Kill Creek watershed

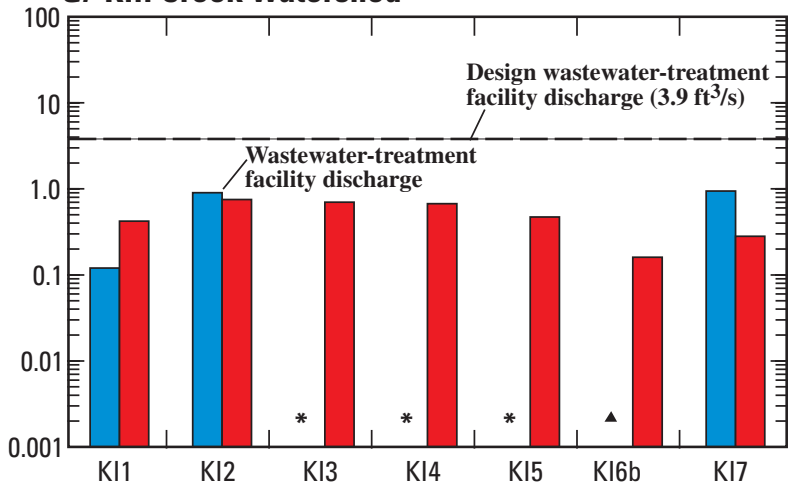

B. Blue River watershed

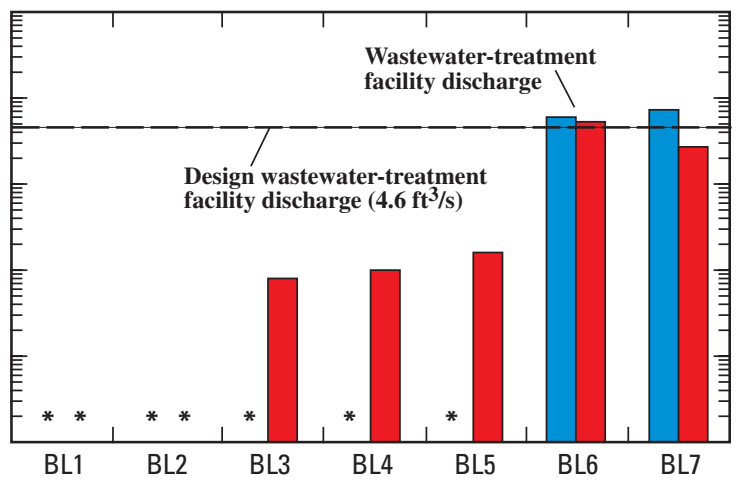

D. Cedar Creek watershed

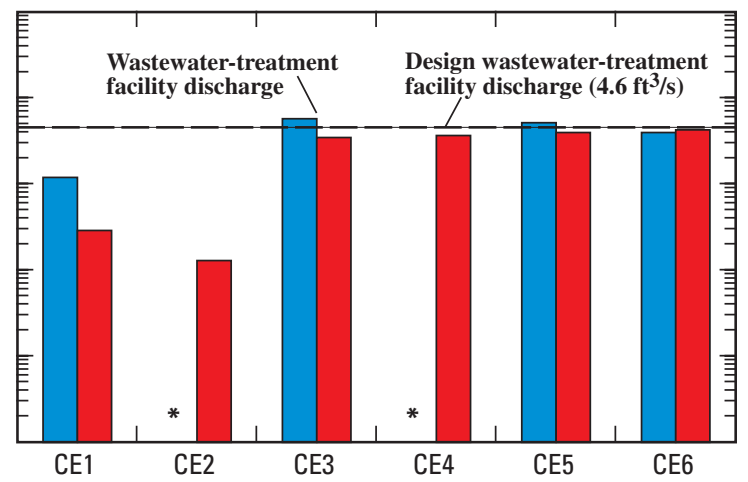

\section{F. Indian Creek watershed}

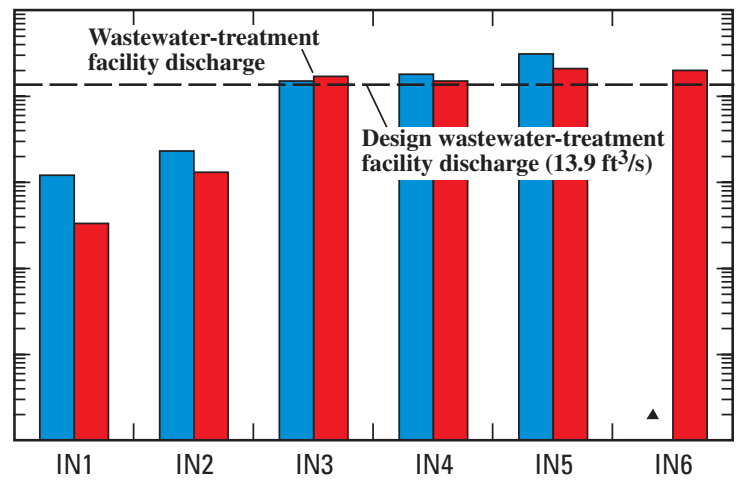

H. Little Bull Creek watershed

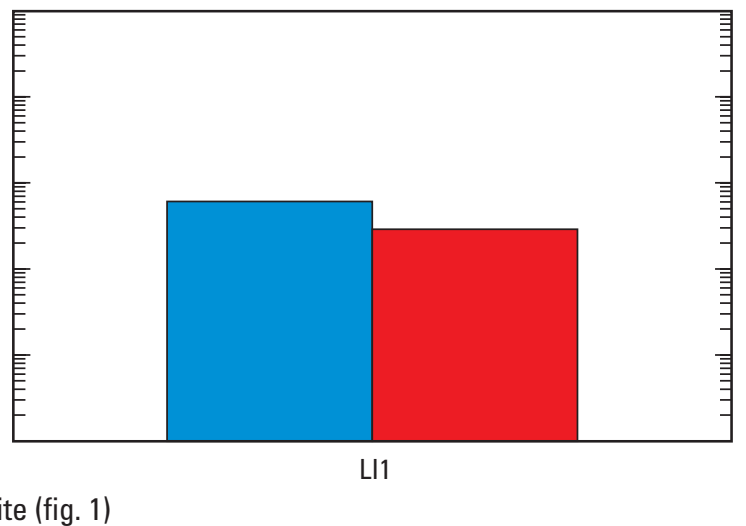

Figure 6. Streamflow at base-flow sampling sites, November 4-7, 2002, and July 14-18, 2003. 


\section{Mill Creek watershed}

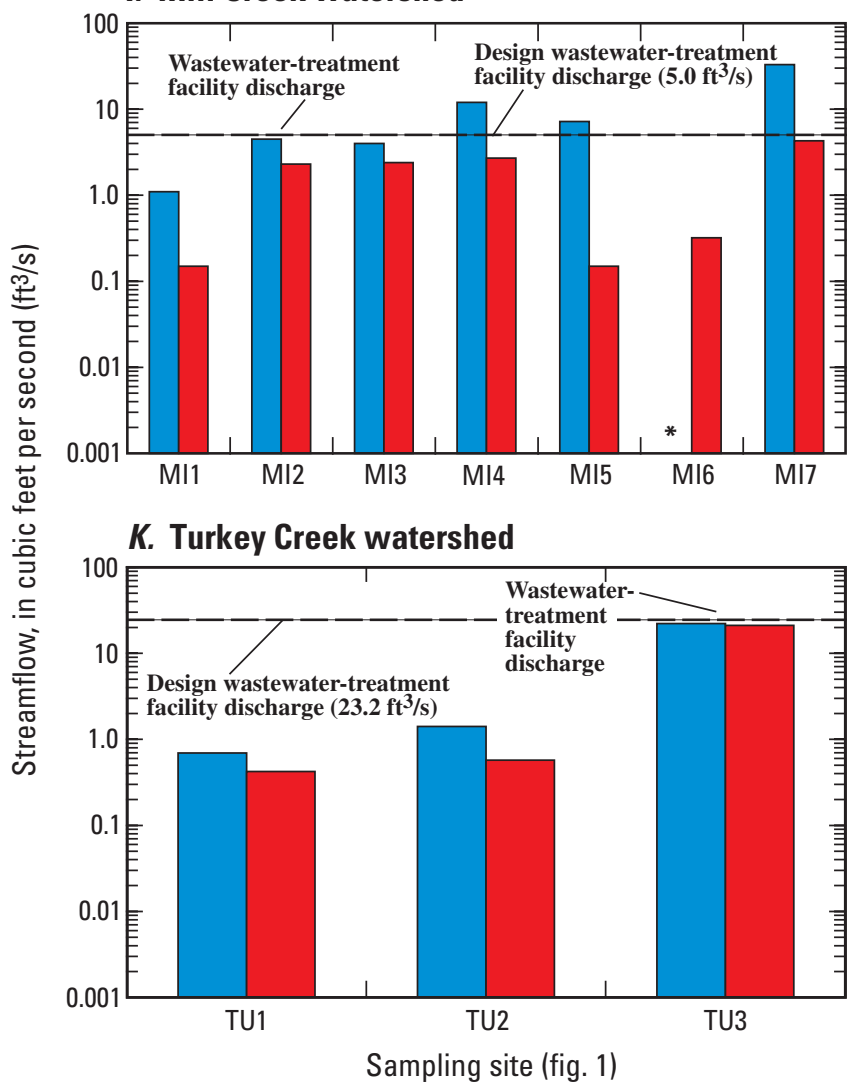

\section{J. Tomahawk Creek watershed}

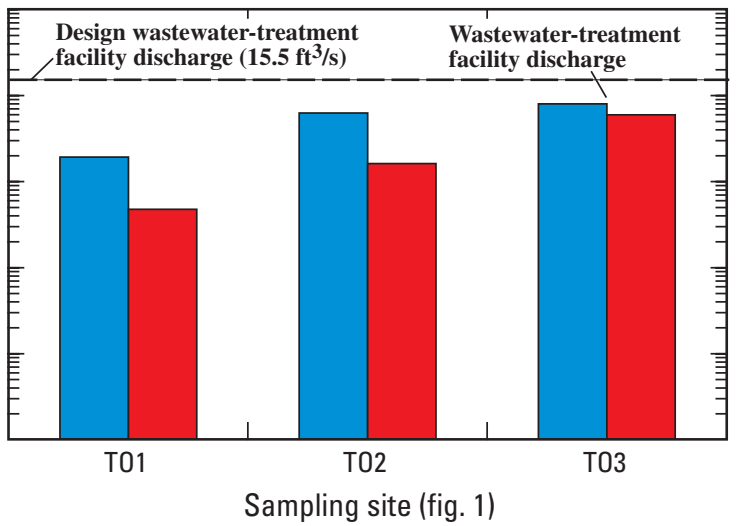

EXPLANATION

Synoptic survey

November 4-7, 2002

July 14-18, 2003

* No observable flow—sample not collected

- Site only sampled during second synoptic survey

Figure 6. Streamflow at base-flow sampling sites, November 4-7, 2002, and July 14-18, 2003.-Continued

\section{Streamflow}

Streamflow measurements were made (or obtained from the WWTF) at the time of sample collection (fig. 6) and were used to estimate sources of streamflow (fig. 7) in Johnson County watersheds as well as to provide an estimate of chemical and biological mass transport through each watershed. Sites BL1 (Coffee Creek at Pflumm Road), BL2 (Wolf Creek at Pflumm Road), and CA1 (Captain Creek near 119th Street) did not have observable streamflow during either synoptic survey. Measurements from three of seven WWTF discharges were larger than the designed flow capacity for the facility (fig. 6). However, designed flow capacity is computed as a daily average, and these measurements were collected during the morning and afternoon when WWTF discharges may be larger than average daily conditions. In six of seven watersheds, WWTF discharges made up the majority of streamflow at the farthest downstream sampling site during base-flow conditions (fig. 7). At three of eight sites, WWTF discharges were greater than 99 percent of the farthest downstream streamflow measurement, indicating possible losses of water due to evaporation or seepage, water use, and (or) errors in streamflow measurements. Streamflow measurements during base-flow conditions indicated that flow conditions were generally consistent downstream from WWTF discharges and that potential waterquality contaminants should not be lost downstream due to dilution of WWTF point sources.

Rainfall of $0.2 \mathrm{in}$. occurred during the base-flow synoptic survey on November 5, 2002, and caused small increases in streamflow at the Blue River and Mill Creek sites. This rainfall could account for net water gains downstream in the Blue River during the November 2002 measurement in contrast to net water losses during the July 2003 measurement (fig. 6). This rainfall also accounted for a $33 \mathrm{ft}^{3} / \mathrm{s}$ measurement of streamflow at site MI7 in November 2002 compared with a $4.3 \mathrm{ft}^{3} / \mathrm{s}$ measurement during base-flow conditions on July 16, 2003. In July 2003, total precipitation for the previous 3 months was at least 3 in. greater than before the initial November synoptic survey (Overland Park Stormwatch, 2004). This may account for a slight net water gain at sites downstream from the WWTF discharge in the Cedar Creek watershed during the July 2003 survey, whereas water was lost downstream during the November 2002 synoptic survey.

Stormflow samples are designated as all samples that were collected at streamflow values larger than $40 \mathrm{ft}^{3} / \mathrm{s}$ to facilitate comparisons between base- and stormflow conditions. Streamflow at the time of stormflow sample collection was a function 


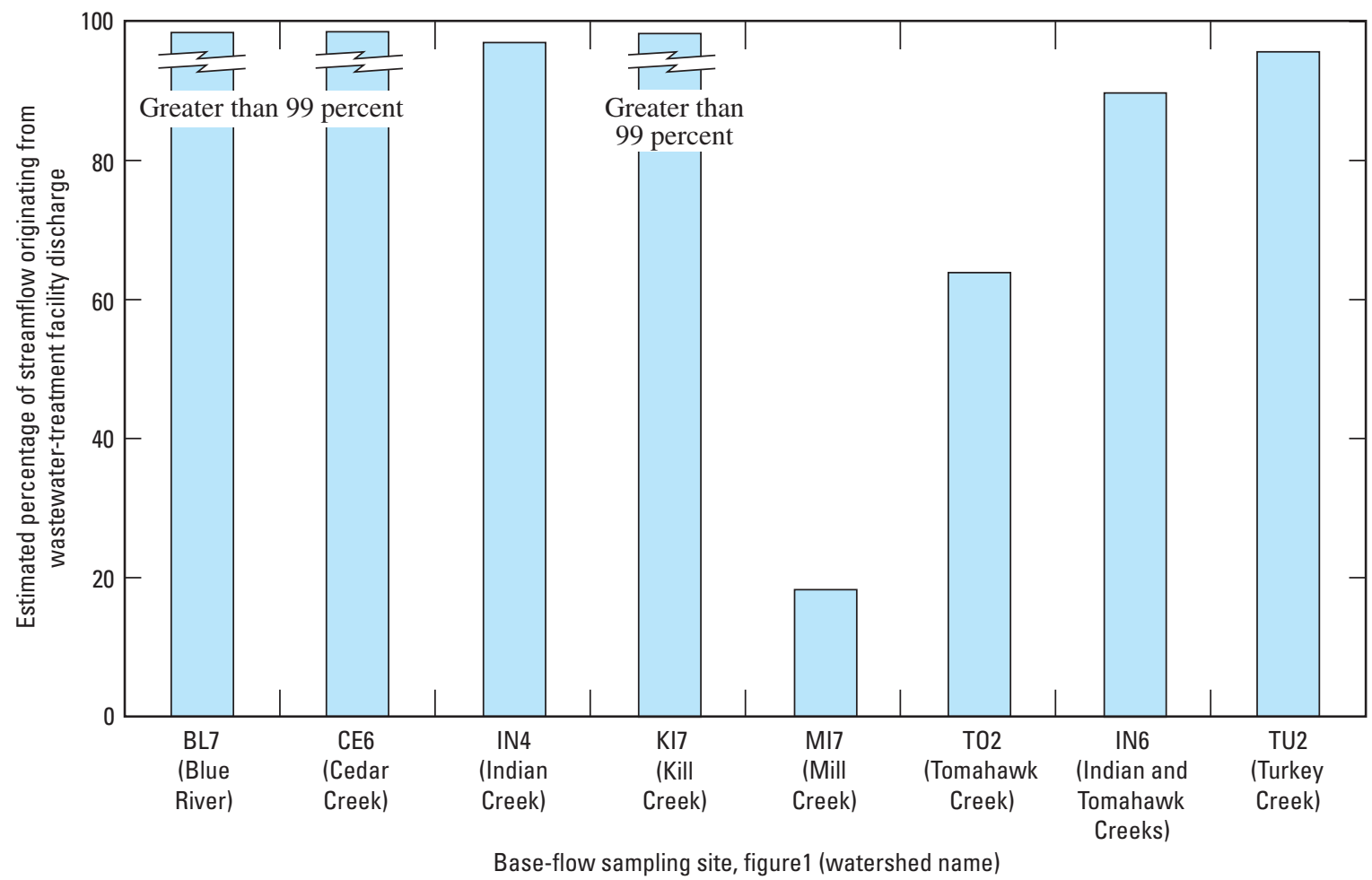

Figure 7. Estimated mean percentage of streamflow at the farthest downstream sampling site originating from wastewater-treatment facility discharges during base-flow sample collection, November 4-7, 2002, and July 14$18,2003$.

of the amount and intensity of precipitation, watershed size, and the extent of impervious surfaces and the number of surfacewater impoundments in each watershed. Site TU1 had the smallest maximum streamflow associated with stormflow samples (table 9) and the smallest watershed size (table 2). Although six base- and stormflow samples were collected from site TU1, streamflow was not measured for one of these samples, thus this sample is not included in figures comparing constituent concentrations to streamflow conditions. In addition to stormflow samples and synoptic base-flow samples, three additional base-flow samples collected at site CE6, one additional base-flow sample collected at site IN6, and two additional base-flow samples collected at site MI7 are included in data analysis to facilitate comparisons between base- and stormflow conditions. An additional base-flow sample was collected at site CE6 after a rainfall of 1.3 in. Although streamflow conditions were small enough $\left(37 \mathrm{ft}^{3} / \mathrm{s}\right)$ to categorize this sample as collected during base-flow conditions (less than $40 \mathrm{ft}^{3} / \mathrm{s}$ ), this sample was affected by storm runoff.

\section{Stream-Water-Quality Conditions}

Complete results of analysis of all stream-water-quality samples are posted on the USGS Web site (http://ks.water.usgs.gov/Kansas/studies/qw/joco) and are on file with USGS in Lawrence, Kansas.

\section{Suspended Sediment}

Suspended sediment is known to affect water clarity, bridge scour, and reservoir sedimentation. Many contaminants such as nutrients, trace elements, bacteria, and organic compounds are known to adsorb to and are transported by suspended sediment (U.S. Environmental Protection Agency, 2000a). Potential sources of suspended sediment include surface runoff, erosion of channel bank material, and resuspension of streambed sediment.

Suspended-sediment samples were collected during both synoptic surveys. All 73 base-flow samples contained less than $50 \mathrm{mg} / \mathrm{L}$ suspended sediment with the exception of the sample from site LI1 collected during the July 2003 synoptic survey $(220 \mathrm{mg} / \mathrm{L})$. The large suspended-sediment concentration in the sample from site LI1 may be due to water spraying on the gravel road (noticed by field personnel) and subsequent runoff into the creek at this site during sample collection; the suspendedsediment concentration was only $11 \mathrm{mg} / \mathrm{L}$ in the sample collected from this site during the November 2002 survey. WWTF sites throughout the county had among the smallest suspendedsediment concentrations in each respective watershed and had a diluting effect on downstream sites. Other than the LI1 site, seven of the eight largest suspended-sediment concentrations were sampled at sites upstream from WWTF discharge locations. 
Table 9. Statistical summary of the results of streamflow measurements collected at Johnson County stormflow sampling sites, northeastern Kansas, October 2002-June 2004.

\begin{tabular}{lcccc}
\hline $\begin{array}{c}\text { Sampling- } \\
\text { site } \\
\begin{array}{c}\text { identifier } \\
\text { (fig. 1) }\end{array}\end{array}$ & $\begin{array}{c}\text { Number of } \\
\text { measure- } \\
\text { ments }\end{array}$ & \multicolumn{2}{c}{ Streamflow (cubic feet per second) } \\
\cline { 3 - 5 } BL5 & 9 & 0.2 & 627 & 10,600 \\
CE6 & 11 & 2.2 & 64 & 1,460 \\
IN6 & 10 & 20 & 1,130 & 5,290 \\
KI6b & 8 & .2 & 213 & 3,010 \\
MI7 & 14 & 4.3 & 330 & 1,030 \\
& & & & \\
TU1 & 5 & .4 & 117 & 337 \\
\hline
\end{tabular}

Suspended-sediment concentrations and loads were significantly larger ( $\mathrm{p}$ value less than 0.01 ) in stormflow samples compared to base-flow samples, and concentrations often paralleled increases in streamflow (fig. 8, fig. 9, table 10). Correlation analyses using Kendall's tau showed a significant positive relation ( $\mathrm{p}$ value less than 0.01 ) between streamflow and suspended-sediment concentration for all samples from stormflow sampling sites (fig. 8). Although suspended-sediment concentrations generally increased with streamflow, precipitation intensity, antecedent moisture conditions, whether the sample was collected on the rising or falling limb of a stormflow hydrograph, and other factors can play a role in determining sediment transport.

\section{Dissolved Solids and Major lons}

Dissolved solids are an important water-quality indicator. In uncontaminated stream water, dissolved solids are the result of the natural dissolution of rocks and minerals (Hem, 1992). The major cations (calcium, magnesium, sodium, and potassium) and anions (sulfate, chloride, fluoride, and silica) are present in different combinations in stream water depending upon the types and amounts of natural and human-related sources. Dissolved solids also are an important indicator of the suitability of water for drinking, irrigation, and industrial use (Maidment, 1993). Concentrations of dissolved solids in stream water are known to vary considerably. Rainwater typically contains dissolved-solids concentrations of $10 \mathrm{mg} / \mathrm{L}$, slightly saline water ranges from 1,000 to $3,000 \mathrm{mg} / \mathrm{L}$, and seawater typically contains dissolved-solids concentrations exceeding 35,000 mg/L (Maidment, 1993). Point-source discharges from WWTFs and runoff from urban areas can increase dissolved-solid concentrations in stream water (Pope and Putnam, 1997).

Base-flow samples from sites located at or immediately downstream from WWTFs typically had the largest dissolvedsolids concentrations in each watershed studied (table 11). Twenty-nine of 73 samples exceeded the USEPA Secondary Drinking-Water Regulation of $500 \mathrm{mg} / \mathrm{L}$ for dissolved solids (U.S. Environmental Protection Agency, 2002); 22 of the 29 samples were collected at or at the next site downstream from a WWTF discharge. The USEPA criterion is not regulatory and is used in this report for comparative purposes only. Although sampling site CE1 was located upstream from WWTF discharges, a base-flow sample from this site contained

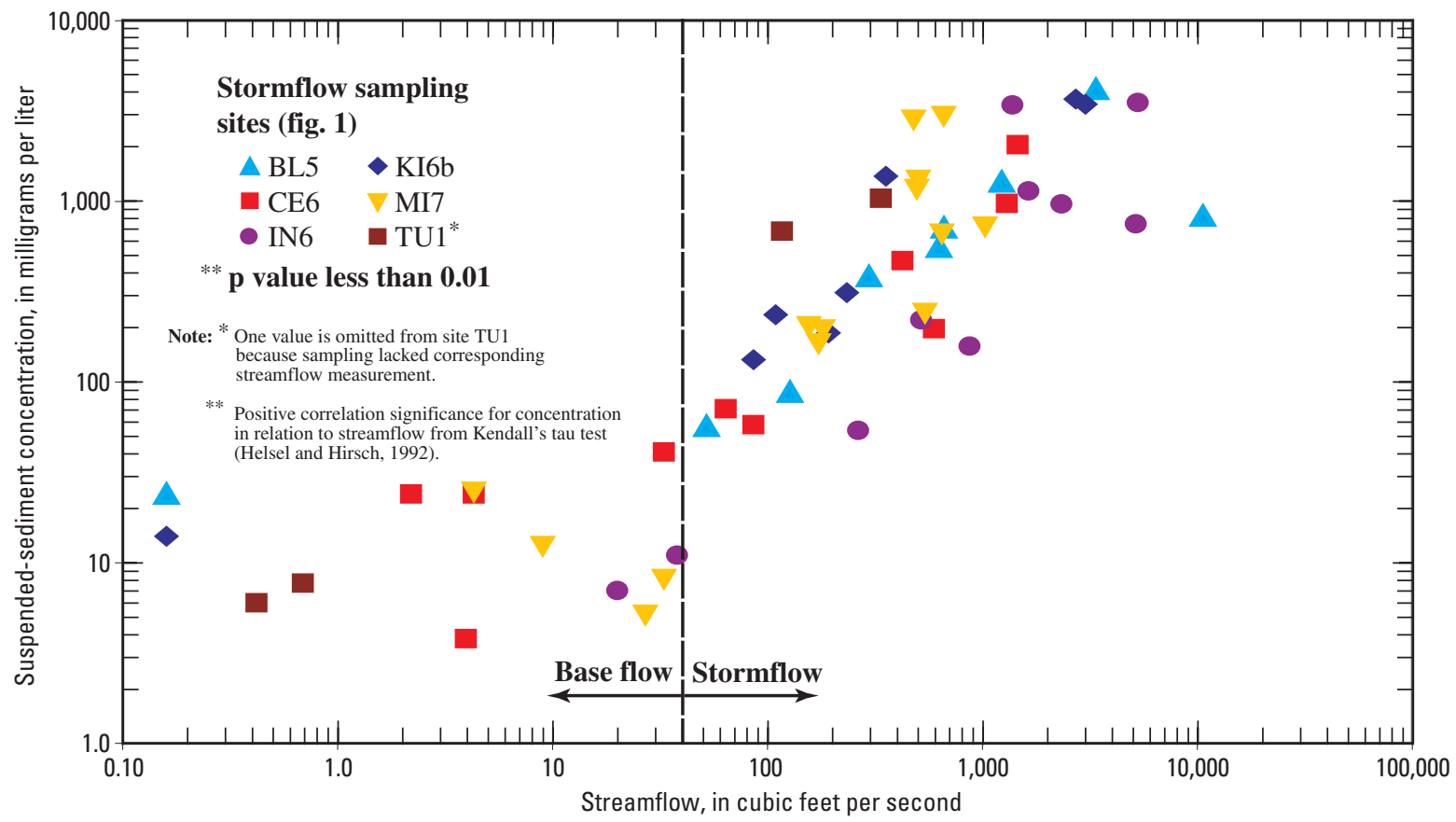

Figure 8. Suspended-sediment concentrations in base- and stormflow samples collected at stormflow sampling sites, October 2002-June 2004. 

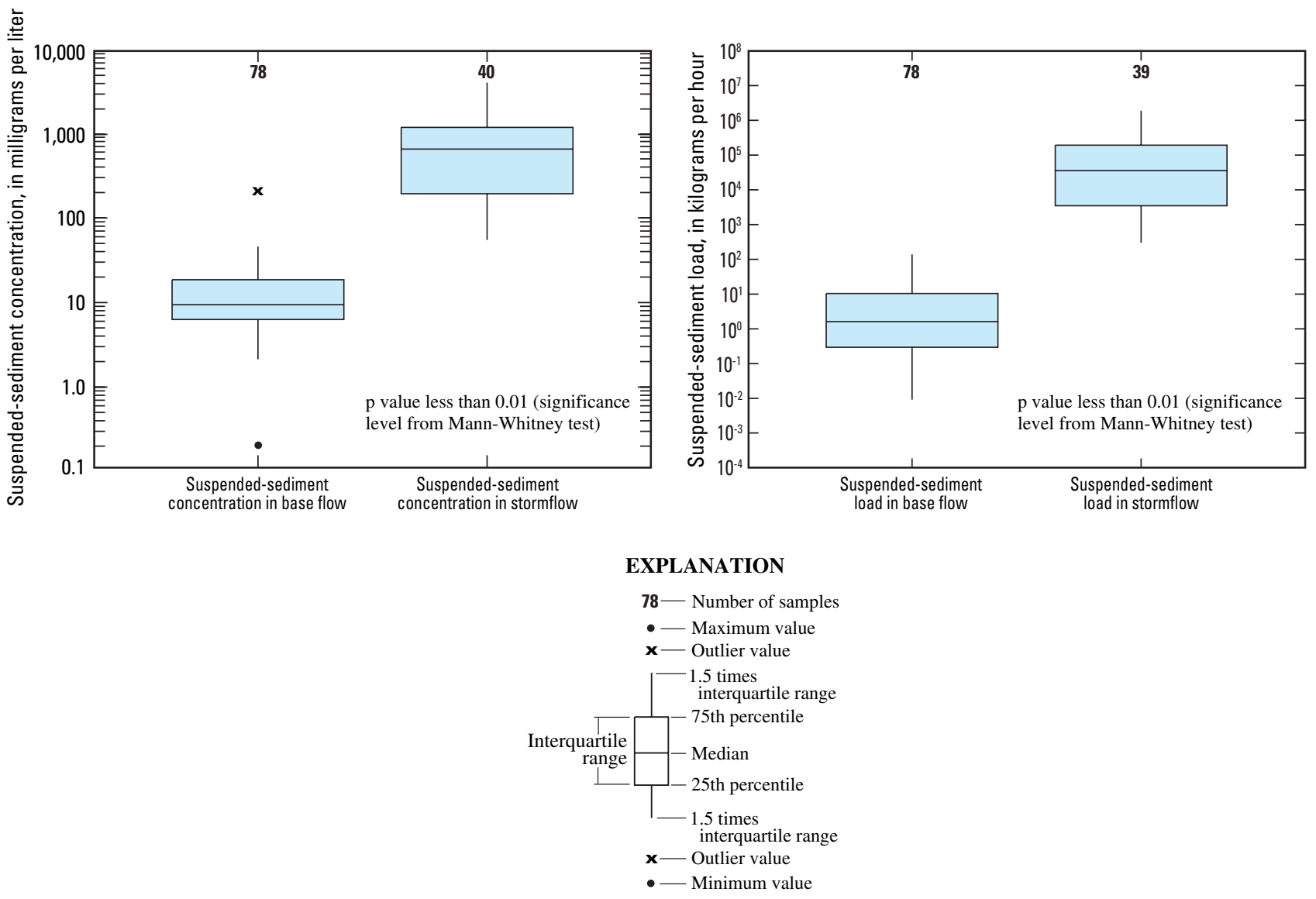

Figure 9. Suspended-sediment concentrations and estimated loads in base- and stormflow samples, October 2002-June 2004.

Table 10. Statistical summary of the results of analysis of suspended sediment in samples collected from Johnson County stormflow sampling sites, northeastern Kansas, October 2002June 2004.

\begin{tabular}{lrrrr}
\hline $\begin{array}{c}\text { Sampling } \\
\text { site } \\
\text { identifier } \\
\text { (fig. 1) }\end{array}$ & $\begin{array}{c}\text { Number of } \\
\text { samples }\end{array}$ & \multicolumn{3}{c}{$\begin{array}{c}\text { Suspended-sediment concentration } \\
\text { (milligrams per liter) }\end{array}$} \\
\cline { 3 - 5 } & & Minimum & Median & Maximum \\
\hline BL5 & 9 & 24 & 558 & 4,170 \\
CE6 & 10 & 4 & 65 & 2,060 \\
IN6 & 10 & 7 & 486 & 3,530 \\
KI6b & 8 & 14 & 275 & 3,690 \\
MI7 & 14 & 5 & 216 & 2,890 \\
& & & & \\
TU1 & 5 & 6 & 320 & 1,040 \\
\hline
\end{tabular}

the largest dissolved-solids concentration $(1,100 \mathrm{mg} / \mathrm{L})$ of all base-flow samples. Site CE1 is located immediately downstream from a limestone quarry, which may have contributed to increased dissolution of bedrock materials. Samples from site CE1 had more than twice the sulfate concentrations of samples from any other site in the county and had among the largest concentrations of calcium, magnesium, and sodium. Sodium, potassium, sulfate, and chloride concentrations generally were found at the largest concentrations in samples from WWTF discharges, whereas calcium and magnesium concentrations in WWTF discharge were similar to concentrations in samples from sites upstream from WWTF discharges.

Dissolved-solid concentrations (fig. 10) generally were diluted with increasing streamflow at stormflow sampling sites. Kendall's tau correlation tests on dissolved solids, magnesium, sodium, and chloride showed significant ( $\mathrm{p}$ values less than $0.01)$ negative correlations with streamflow for all combined values. Exceptions to the diluting trend include two samples from Cedar Creek (site CE6), one from Indian Creek (site IN6), and two samples from Mill Creek (site MI7) collected during the winter, which likely were affected by road-salt application. These samples had the largest concentrations of magnesium, sodium, and chloride of all stormflow samples collected. A sample collected from site IN6 on January 26, 2004, had the largest concentrations of dissolved solids and major ions, including a chloride concentration of $1,000 \mathrm{mg} / \mathrm{L}$ (table 12), which exceeded the KDHE acute aquatic-life use criterion $(860 \mathrm{mg} / \mathrm{L})$ (Kansas Department of Health and Environment, 2004c). The large concentration of chloride at this site is likely 
Table 11. Results of analysis of dissolved solids and major ions in water from base-flow sampling sites, Johnson County, northeastern Kansas, November 4-7, 2002, and July 14-18, 2003.

[All concentrations are given in milligrams per liter]

\begin{tabular}{|c|c|c|c|c|c|c|c|c|c|c|}
\hline \multirow{2}{*}{$\begin{array}{l}\text { Sampling } \\
\text { site } \\
\text { identifier } \\
\text { (fig. 1) }\end{array}$} & \multirow{2}{*}{$\begin{array}{l}\text { Date of sample } \\
\text { (month/day/year) }\end{array}$} & \multicolumn{9}{|c|}{ Concentrations } \\
\hline & & $\begin{array}{l}\text { Dissolved } \\
\text { solids }\end{array}$ & Calcium & Magnesium & Sodium & Potassium & Sulfate & Chloride & Fluoride & Silica \\
\hline \multicolumn{11}{|c|}{ Big Bull Creek watershed } \\
\hline \multirow[t]{2}{*}{ BI1 } & $11 / 4 / 02$ & 500 & 60 & 12 & 73 & 12 & 120 & 90 & 0.5 & 3.0 \\
\hline & $7 / 14 / 03$ & 450 & 63 & 9.7 & 60 & 9.0 & 68 & 60 & .7 & 3.9 \\
\hline \multicolumn{11}{|c|}{ Blue River watershed } \\
\hline BL3 & $7 / 18 / 03$ & 330 & 58 & 14 & 78 & 13 & 43 & 40 & .4 & 4.6 \\
\hline BL4 & $7 / 18 / 03$ & 320 & 30 & 10 & 40 & 5.0 & 130 & 30 & .6 & 3.8 \\
\hline BL5 & $7 / 18 / 03$ & 340 & 60 & 9.7 & 25 & 3.0 & 46 & 50 & .4 & 3.7 \\
\hline \multirow[t]{2}{*}{ BL6 } & $11 / 4 / 02$ & 630 & 58 & 20 & 92 & 11 & 170 & 80 & .4 & 11 \\
\hline & $7 / 18 / 03$ & 600 & 61 & 9.7 & 23 & 4.0 & 130 & 80 & .5 & 3.5 \\
\hline \multirow[t]{2}{*}{ BL7 } & $11 / 4 / 02$ & 540 & 70 & 16 & 60 & 7.5 & 130 & 60 & .4 & 7.3 \\
\hline & $7 / 18 / 03$ & 530 & 63 & 14 & 69 & 9.0 & 120 & 80 & .5 & 3.1 \\
\hline \multirow[t]{2}{*}{ BR1 } & $11 / 7 / 02$ & 210 & 34 & 5.4 & 22 & 4.0 & 45 & 30 & .3 & 4.5 \\
\hline & $7 / 15 / 03$ & 370 & 60 & 7.7 & 37 & 5.0 & 48 & 70 & .3 & 3.0 \\
\hline \multirow[t]{2}{*}{ BR2 } & 11/7/02 & 240 & 41 & 5.8 & 24 & 3.5 & 43 & 30 & .2 & 3.8 \\
\hline & $7 / 15 / 03$ & 280 & 46 & 6.1 & 30 & 4.0 & 32 & 50 & .3 & 2.1 \\
\hline \multicolumn{11}{|c|}{ Cedar Creek watershed } \\
\hline \multirow[t]{2}{*}{ CE1 } & $11 / 4 / 02$ & 1,100 & 100 & 33 & 150 & 6.1 & 410 & 50 & .7 & 3.4 \\
\hline & $7 / 14 / 03$ & 840 & 99 & 24 & 100 & 6.0 & 400 & 50 & .6 & 1.8 \\
\hline CE2 & $7 / 14 / 03$ & 450 & 90 & 9.7 & 37 & 3.0 & 77 & 60 & .8 & 4.4 \\
\hline \multirow[t]{2}{*}{ CE3 } & $11 / 4 / 02$ & 640 & 64 & 17 & 100 & 13 & 140 & 120 & .7 & 11 \\
\hline & $7 / 14 / 03$ & 640 & 71 & 14 & 110 & 14 & 130 & 120 & .7 & 4.4 \\
\hline CE4 & $7 / 14 / 03$ & 630 & 69 & 14 & 100 & 13 & 130 & 120 & .7 & 4.0 \\
\hline \multirow[t]{2}{*}{ CE5 } & $11 / 4 / 02$ & 530 & 77 & 14 & 64 & 7.0 & 110 & 70 & .5 & 7.1 \\
\hline & $7 / 14 / 03$ & 480 & 62 & 8.8 & 69 & 7.0 & 110 & 80 & .5 & 3.3 \\
\hline
\end{tabular}


2002, and July 14-18, 2003.-Continued

[All concentrations are given in milligrams per liter]

\begin{tabular}{|c|c|c|c|c|c|c|c|c|c|c|}
\hline \multirow{2}{*}{$\begin{array}{c}\text { Sampling site } \\
\text { (fig. 1) }\end{array}$} & \multirow{2}{*}{$\begin{array}{c}\text { Date of sample } \\
\text { (month/day/year) }\end{array}$} & \multicolumn{9}{|c|}{ Concentrations } \\
\hline & & $\begin{array}{l}\text { Dissolved } \\
\text { solids }\end{array}$ & Calcium & Magnesium & Sodium & Potassium & Sulfate & Chloride & Fluoride & Silica \\
\hline \multicolumn{11}{|c|}{ Cedar Creek watershed-Continued } \\
\hline \multirow[t]{2}{*}{ CE6 } & $11 / 4 / 02$ & 460 & 71 & 13 & 54 & 6.5 & 98 & 60 & 0.4 & 6.6 \\
\hline & $7 / 14 / 03$ & 330 & 50 & 7.2 & 42 & 6.0 & 64 & 50 & .4 & 2.3 \\
\hline \multicolumn{11}{|c|}{ Dykes Branch watershed } \\
\hline \multirow[t]{2}{*}{ DY1 } & $11 / 7 / 02$ & 370 & 52 & 6.7 & 51 & 4.4 & 120 & 40 & .6 & 6.6 \\
\hline & $7 / 17 / 03$ & 450 & 58 & 7.4 & 64 & 6.0 & 100 & 80 & .6 & 2.4 \\
\hline \multicolumn{11}{|c|}{ Indian Creek watershed } \\
\hline \multirow[t]{2}{*}{ IN1 } & $11 / 6 / 02$ & 350 & 56 & 11 & 35 & 3.1 & 70 & 40 & .3 & 2.3 \\
\hline & 7/17/03 & 500 & 68 & 16 & 51 & 3.0 & 89 & 110 & .4 & 1.3 \\
\hline \multirow[t]{2}{*}{ IN2 } & $11 / 6 / 02$ & 440 & 74 & 13 & 38 & 3.1 & 79 & 60 & .3 & 6.0 \\
\hline & 7/17/03 & 430 & 60 & 12 & 41 & 3.0 & 86 & 70 & .4 & 1.6 \\
\hline \multirow[t]{2}{*}{ IN3 } & $11 / 6 / 02$ & 350 & 52 & 17 & 110 & 14 & 130 & 110 & .7 & 15 \\
\hline & 7/17/03 & 640 & 49 & 12 & 100 & 14 & 120 & 110 & .7 & 5.6 \\
\hline \multirow[t]{2}{*}{ IN4 } & $11 / 6 / 02$ & 590 & 58 & 16 & 95 & 11 & 120 & 100 & .6 & 13 \\
\hline & 7/17/03 & 620 & 51 & 12 & 97 & 14 & 120 & 100 & .7 & 5.5 \\
\hline \multirow[t]{2}{*}{ IN5 } & $11 / 6 / 02$ & 550 & 65 & 15 & 73 & 7.7 & 120 & 80 & .4 & 9.0 \\
\hline & $7 / 17 / 03$ & 600 & 54 & 13 & 89 & 13 & 130 & 100 & .6 & 4.2 \\
\hline IN6 & 7/17/03 & 590 & 56 & 13 & 90 & 13 & 130 & 110 & .6 & 4.0 \\
\hline \multicolumn{11}{|c|}{ Kill Creek watershed } \\
\hline \multirow[t]{2}{*}{ KI1 } & $11 / 4 / 02$ & 470 & 86 & 15 & 34 & 3.8 & 120 & 30 & .3 & 7.9 \\
\hline & $7 / 15 / 03$ & 420 & 84 & 15 & 28 & 3.0 & 93 & 20 & .4 & 3.6 \\
\hline \multirow[t]{2}{*}{$\mathrm{KI} 2$} & $11 / 4 / 02$ & 500 & 58 & 11 & 66 & 13 & 70 & 70 & .8 & 7.3 \\
\hline & $7 / 15 / 03$ & 450 & 56 & 9.9 & 66 & 13 & 68 & 80 & .9 & 3.8 \\
\hline $\mathrm{KI} 3$ & $7 / 15 / 03$ & 460 & 60 & 11 & 60 & 11 & 72 & 80 & .9 & 3.3 \\
\hline KI4 & $7 / 15 / 03$ & 330 & 52 & 8 & 37 & 7.0 & 52 & 40 & .7 & 3.3 \\
\hline KI5 & $7 / 15 / 03$ & 390 & 63 & 8.8 & 45 & 8.0 & 60 & 60 & .6 & 2.9 \\
\hline
\end{tabular}


Table 11. Results of analysis of dissolved solids and major ions in base-flow samples collected from selected Johnson County streams, northeastern Kansas, November 4-7, 2002, and July 14-18, 2003.-Continued

[All concentrations are given in milligrams per liter]

\begin{tabular}{|c|c|c|c|c|c|c|c|c|c|c|}
\hline \multirow{2}{*}{$\begin{array}{c}\text { Sampling site } \\
\text { (fig. 1) }\end{array}$} & \multirow{2}{*}{$\begin{array}{c}\text { Date of sample } \\
\text { (month/day/year) }\end{array}$} & \multicolumn{9}{|c|}{ Concentrations } \\
\hline & & $\begin{array}{l}\text { Dissolved } \\
\text { solids }\end{array}$ & Calcium & Magnesium & Sodium & Potassium & Sulfate & Chloride & Fluoride & Silica \\
\hline \multicolumn{11}{|c|}{ Kill Creek watershed_-Continued } \\
\hline KI6b & $7 / 15 / 03$ & 310 & 61 & 9.6 & 23 & 4.0 & 47 & 30 & 0.4 & 4.1 \\
\hline \multirow[t]{2}{*}{ KI7 } & $11 / 4 / 02$ & 340 & 72 & 11 & 14 & 3.6 & 84 & 20 & .2 & 5.6 \\
\hline & $7 / 15 / 03$ & 330 & 66 & 10 & 20 & 4.0 & 45 & 30 & .3 & 4.6 \\
\hline \multicolumn{11}{|c|}{ Little Bull Creek watershed } \\
\hline \multirow[t]{2}{*}{ LI1 } & $11 / 4 / 02$ & 520 & 43 & 9.2 & 100 & 12 & 70 & 130 & .3 & 6.0 \\
\hline & $7 / 14 / 03$ & 350 & 41 & 5.8 & 56 & 8.0 & 38 & 70 & .4 & 2.9 \\
\hline \multicolumn{11}{|c|}{ Mill Creek watershed } \\
\hline \multirow[t]{2}{*}{ MI1 } & $11 / 4 / 02$ & 270 & 37 & 7.3 & 34 & 4.2 & 46 & 50 & .3 & 2.4 \\
\hline & $7 / 16 / 03$ & 430 & 59 & 9.9 & 57 & 4.0 & 65 & 90 & .5 & 2.4 \\
\hline \multirow[t]{2}{*}{ MI2 } & $11 / 4 / 02$ & 720 & 46 & 16 & 130 & 16 & 120 & 170 & .7 & 13 \\
\hline & $7 / 16 / 03$ & 860 & 55 & 12 & 170 & 15 & 120 & 260 & .7 & 4.8 \\
\hline \multirow[t]{2}{*}{ MI3 } & $11 / 4 / 02$ & 620 & 45 & 14 & 110 & 14 & 96 & 150 & .6 & 9.6 \\
\hline & $7 / 16 / 03$ & 900 & 60 & 12 & 170 & 13 & 120 & 280 & .7 & 4.2 \\
\hline \multirow[t]{2}{*}{ MI4 } & $11 / 4 / 02$ & 570 & 72 & 17 & 73 & 6.5 & 120 & 110 & .4 & 6.0 \\
\hline & $7 / 16 / 03$ & 640 & 75 & 13 & 94 & 8.0 & 110 & 140 & .5 & 2.5 \\
\hline \multirow[t]{2}{*}{ MI5 } & $11 / 4 / 02$ & 500 & 94 & 13 & 39 & 2.4 & 90 & 60 & .2 & 6.8 \\
\hline & $7 / 16 / 03$ & 330 & 65 & 7 & 28 & 3.0 & 43 & 50 & .3 & 2.7 \\
\hline MI6 & $7 / 16 / 03$ & 470 & 87 & 13 & 34 & 2.0 & 74 & 60 & .4 & 3.8 \\
\hline \multirow[t]{2}{*}{ MI7 } & $11 / 4 / 02$ & 430 & 69 & 12 & 47 & 4.0 & 74 & 80 & .3 & 6.0 \\
\hline & $7 / 16 / 03$ & 260 & 44 & 6.2 & 28 & 4.0 & 40 & 40 & .3 & 1.7 \\
\hline \multicolumn{11}{|c|}{ Tomahawk Creek watershed } \\
\hline \multirow[t]{2}{*}{ TO1 } & $11 / 6 / 02$ & 520 & 100 & 18 & 36 & 2.8 & 99 & 40 & .3 & 8.3 \\
\hline & $7 / 17 / 03$ & 530 & 88 & 17 & 47 & 3.0 & 110 & 60 & .5 & 1.7 \\
\hline \multirow[t]{2}{*}{ TO2 } & $11 / 6 / 02$ & 460 & 82 & 15 & 40 & 3.2 & 98 & 46 & .3 & 6.8 \\
\hline & 7/17/03 & 440 & 64 & 13 & 42 & 3.0 & 82 & 60 & .4 & 1.5 \\
\hline
\end{tabular}


Table 11. Results of analysis of dissolved solids and major ions in base-flow samples collected from selected Johnson County streams, northeastern Kansas, November 4-7, 2002, and July 14-18, 2003.-Continued

[All concentrations are given in milligrams per liter]

\begin{tabular}{|c|c|c|c|c|c|c|c|c|c|c|}
\hline \multirow{2}{*}{$\begin{array}{l}\text { Sampling site } \\
\text { (fig. 1) }\end{array}$} & \multirow{2}{*}{$\begin{array}{c}\text { Date of sample } \\
\text { (month/day/year) }\end{array}$} & \multicolumn{9}{|c|}{ Concentrations } \\
\hline & & $\begin{array}{l}\text { Dissolved } \\
\text { solids }\end{array}$ & Calcium & Magnesium & Sodium & Potassium & Sulfate & Chloride & Fluoride & Silica \\
\hline \multicolumn{11}{|c|}{ Tomahawk Creek watershed_Continued } \\
\hline \multirow[t]{2}{*}{ TO3 } & $11 / 6 / 02$ & 690 & 48 & 20 & 110 & 13 & 180 & 110 & 0.6 & 13 \\
\hline & $7 / 17 / 03$ & 620 & 48 & 14 & 98 & 15 & 150 & 100 & .5 & 5.5 \\
\hline \multicolumn{11}{|c|}{ Turkey Creek watershed } \\
\hline \multirow[t]{2}{*}{ TU1 } & $11 / 7 / 02$ & 340 & 58 & 8 & 38 & 2.5 & 47 & 70 & .2 & 4.3 \\
\hline & $7 / 16 / 03$ & 540 & 71 & 13 & 74 & 5.0 & 100 & 140 & .4 & 2.9 \\
\hline \multirow[t]{2}{*}{ TU2 } & $11 / 7 / 02$ & 400 & 64 & 8.9 & 47 & 2.6 & 54 & 90 & .2 & 3.0 \\
\hline & $7 / 16 / 03$ & 420 & 63 & 8.9 & 59 & 4.0 & 57 & 110 & .3 & 2.3 \\
\hline \multirow[t]{2}{*}{ TU3 } & 11/7/02 & 350 & 48 & 16 & 110 & 12 & 180 & 100 & .6 & 12 \\
\hline & $7 / 16 / 03$ & 550 & 46 & 10 & 88 & 14 & 100 & 110 & .6 & 5.5 \\
\hline
\end{tabular}


A. Dissolved solids

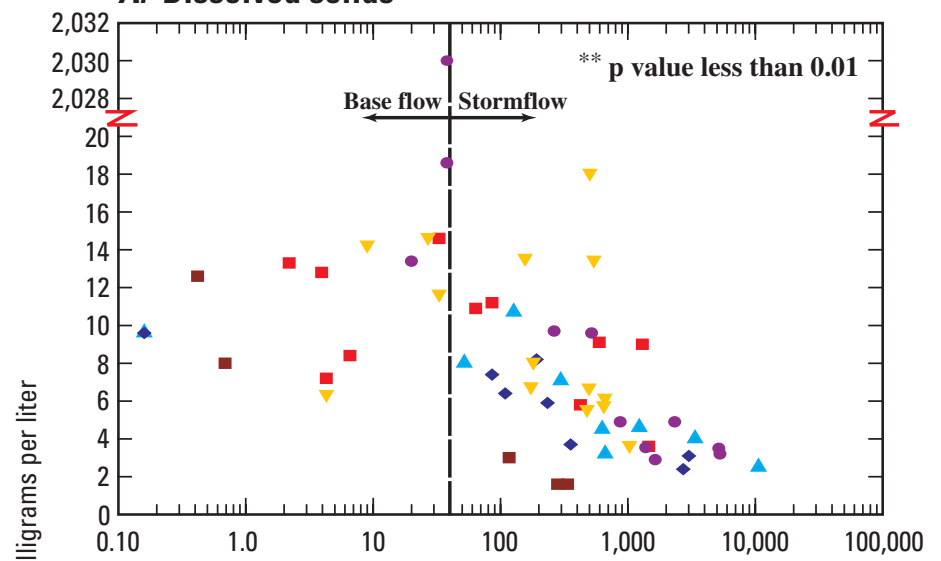

C. Sodium

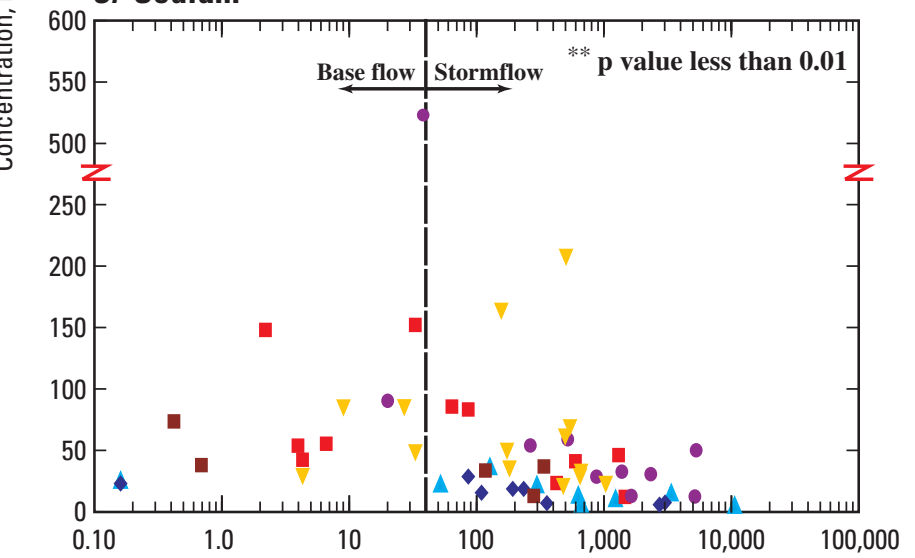

B. Magnesium

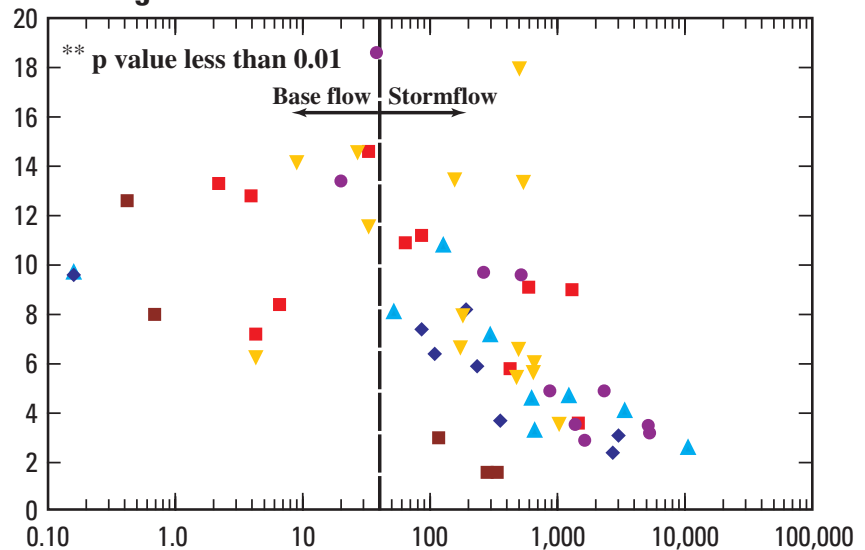

D. Chloride

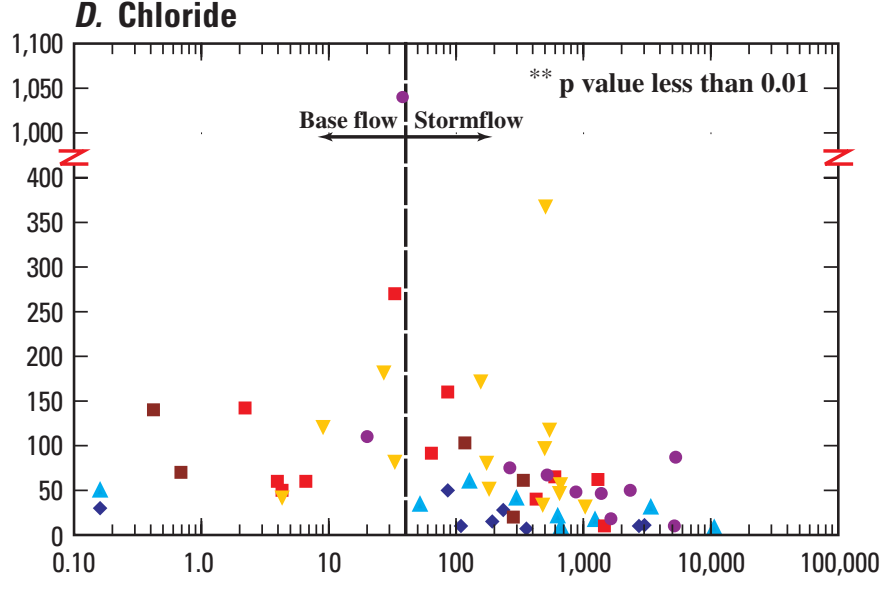

Streamflow, in cubic feet per second

\section{EXPLANATION}

Stormflow sampling sites (fig. 1)

$$
\begin{array}{cc}
\Delta \text { BL5 } & \text { KI6b } \\
\text { CE6 } & \text { MI7 } \\
\text { IN6 } & \text { TU1 }{ }^{*} \\
\text { Note: } & \text { One value is omitted from site TU1 } \\
\text { because sampling lacked corresponding } \\
\text { streamflow measurement. } \\
\text { ** } \\
\text { Negative correlation significance for concentration } \\
\text { in relation to streamflow from Kendall's tau test } \\
\text { (Helsel and Hirsch, 1992). }
\end{array}
$$

Figure 10. Dissolved solids, magnesium, sodium, and chloride concentrations in base- and stormflow samples collected at stormflow sampling sites, October 2002-June 2004.

the result of road-salt application and the large percentage of impervious surface area in the watershed (table 4).

The Blue River and Kill Creek sampling sites (BL5 and KI6b) had smaller dissolved-solids concentrations in stormflow samples collected during winter months and smaller median and maximum concentrations of dissolved solids, sodium, chloride, and sulfate (fig. 10, table 12). This is possibly because of fewer roads, less impervious surface area, and generally smaller contributions from WWTF discharges (fig. 1, table 3). Because potassium, silica, and fluoride did not exhibit discernible differences between base- and stormflow samples, they were not included in figure 10 .

\section{Nutrients}

Nutrients, including nitrogen and phosphorus, are essential to aquatic life. Nutrients support the growth of primary producers that drive the diversity and productivity of aquatic systems. However, excessive levels of nutrients are known to cause eutrophication in surface water, resulting in algal blooms, fish kills, low dissolved oxygen levels, taste-and-odor effects in drinking water, and decreased aquatic species diversity. Typical nutrient sources include municipal wastewater discharge, fertilizers, and runoff from confined animal feeding operations (Masters, 1991). Johnson County data were compared to 
Table 12. Statistical summary of the results of analysis of dissolved solids and major ions collected from Johnson County stormflow sampling sites, northeastern Kansas, October 2002-June 2004.

[All concentrations are reported in milligrams per liter]

\begin{tabular}{|c|c|c|c|c|}
\hline \multirow{2}{*}{$\begin{array}{l}\text { Sampling site identifier } \\
\text { (fig. 1) }\end{array}$} & \multirow{2}{*}{ Number of samples } & \multicolumn{3}{|c|}{ Concentration } \\
\hline & & Minimum & Median & Maximum \\
\hline \multicolumn{5}{|c|}{ Dissolved solids } \\
\hline BL5 & 9 & 170 & 240 & 390 \\
\hline CE6 & 11 & 190 & 420 & 790 \\
\hline IN6 & 10 & 160 & 280 & 2,000 \\
\hline KI6b & 8 & 180 & 230 & 320 \\
\hline MI7 & 14 & 200 & 330 & 960 \\
\hline TU1 & 6 & 130 & 240 & 540 \\
\hline \multicolumn{5}{|c|}{ Calcium } \\
\hline BL5 & 9 & 21 & 46 & 73 \\
\hline CE6 & 11 & 28 & 60 & 73 \\
\hline IN6 & 10 & 21 & 30 & 88 \\
\hline KI6b & 8 & 23 & 40 & 61 \\
\hline MI7 & 14 & 28 & 50 & 110 \\
\hline TU1 & 6 & 15 & 24 & 71 \\
\hline \multicolumn{5}{|c|}{ Magnesium } \\
\hline BL5 & 9 & 2.6 & 4.7 & 11 \\
\hline CE6 & 11 & 3.6 & 9.1 & 15 \\
\hline IN6 & 10 & 2.9 & 4.9 & 19 \\
\hline KI6b & 8 & 2.4 & 6.2 & 9.6 \\
\hline MI7 & 14 & 3.5 & 7.3 & 18 \\
\hline TU1 & 6 & 1.6 & 2.6 & 13 \\
\hline \multicolumn{5}{|c|}{ Sodium } \\
\hline BL5 & 9 & 4.9 & 15 & 34 \\
\hline CE6 & 11 & 12 & 54 & 150 \\
\hline IN6 & 10 & 12 & 41 & 520 \\
\hline KI6b & 8 & 5.8 & 17 & 29 \\
\hline MI7 & 14 & 19 & 47 & 210 \\
\hline TU1 & 6 & 13 & 35 & 74 \\
\hline \multicolumn{5}{|c|}{ Potassium } \\
\hline BL5 & 9 & 3.0 & 3.7 & 4.3 \\
\hline CE6 & 11 & 3.6 & 6.0 & 15 \\
\hline IN6 & 10 & 2.3 & 4.0 & 13 \\
\hline KI6b & 8 & 3.4 & 4.0 & 5.1 \\
\hline MI7 & 14 & 2.9 & 4.0 & 8.3 \\
\hline TU1 & 6 & 2.0 & 2.3 & 5.0 \\
\hline \multicolumn{5}{|c|}{ Sulfate } \\
\hline BL5 & 8 & 11 & 26 & 76 \\
\hline CE6 & 11 & 26 & 93 & 200 \\
\hline IN6 & 10 & 17 & 38 & 130 \\
\hline KI6b & 8 & 16 & 35 & 54 \\
\hline MI7 & 14 & 30 & 53 & 120 \\
\hline TU1 & 6 & 13 & 22 & 100 \\
\hline
\end{tabular}


Table 12. Statistical summary of the results of analysis of dissolved solids and major ions collected from Johnson County stormflow sampling sites, northeastern Kansas, October 2002-June 2004.-Continued

[All concentrations are reported in milligrams per liter]

\begin{tabular}{|c|c|c|c|c|}
\hline \multirow{2}{*}{$\begin{array}{l}\text { Sampling site identifier } \\
\text { (fig. 1) }\end{array}$} & \multirow{2}{*}{ Number of samples } & \multicolumn{3}{|c|}{ Concentration } \\
\hline & & Minimum & Median & Maximum \\
\hline \multicolumn{5}{|c|}{ Chloride } \\
\hline BL5 & 9 & 8.0 & 31 & 60 \\
\hline CE6 & 11 & 10 & 62 & 270 \\
\hline IN6 & 10 & 10 & 59 & 1,000 \\
\hline KI6b & 8 & 7.0 & 13 & 50 \\
\hline MI7 & 14 & 30 & 80 & 370 \\
\hline TU1 & 6 & 20 & 66 & 140 \\
\hline \multicolumn{5}{|c|}{ Fluoride } \\
\hline BL5 & 9 & .2 & .2 & .4 \\
\hline CE6 & 11 & .2 & .3 & 6 \\
\hline IN6 & 10 & .2 & .2 & 6 \\
\hline KI6b & 8 & .2 & .3 & 6 \\
\hline MI7 & 14 & .2 & .3 & .4 \\
\hline TU1 & 6 & .1 & .2 & .4 \\
\hline \multicolumn{5}{|c|}{ Silica } \\
\hline BL5 & 8 & 2.8 & 3.8 & 5.8 \\
\hline CE6 & 11 & 1.8 & 4.4 & 6.6 \\
\hline IN6 & 10 & 1.8 & 3.0 & 6.8 \\
\hline KI6b & 8 & 2.9 & 4.7 & 6.9 \\
\hline MI7 & 14 & 1.5 & 2.7 & 7.4 \\
\hline TU1 & 6 & 1.4 & 2.7 & 4.3 \\
\hline
\end{tabular}

historical data compiled by the USGS National Water-Quality Assessment (NAWQA) program from surface-water samples collected throughout the United States from 1980 to 1990 (U.S. Geological Survey, 2004). NAWQA median and maximum values of frequently analyzed nutrients were grouped by type of sampling site (urban or rural, table 13).

\section{Nitrogen}

Nitrogen occurs in three primary forms in surface waternitrite/nitrate, ammonia/ammonium, and organic nitrogen (Hem, 1992). Nitrate and free ammonia/ammonium are bioavailable forms of nitrogen that are known to enhance plant and algal growth. Free ammonia/ammonium is known to be toxic to fish species (U.S. Environmental Protection Agency, 1999). Additionally, bacteria are known to convert ammonia/ammonium to nitrate in the water column, which depletes stream dissolved oxygen levels and potentially causes fish kills.

The largest mean total nitrogen concentrations (calculated by summing nitrite and nitrate, ammonia, and organic nitrogen concentrations) were determined in WWTF discharges from sites BL6 (19 mg/L), CE3 (9.2 mg/L), IN3 (16 mg/L), MI2
(18 mg/L), TO3 (16 mg/L), and TU3 (19 mg/L) during baseflow synoptic surveys (fig. 11; table 13). The total nitrogen concentration in discharge from the Kill Creek wastewater facility (site KI2) was among the largest in the county during the first synoptic survey $(20 \mathrm{mg} / \mathrm{L})$; however, this facility had just begun operation in October 2002, and bacterial processes were not mature in activated sludge (E. Hack, Johnson County Wastewater and Public Works, written commun, 2005). The total nitrogen concentration was the smallest of all WWTF discharge samples from site KI2 during the July 2003 synoptic survey $(3.0 \mathrm{mg} / \mathrm{L})$. Total nitrogen concentrations decreased downstream from WWTF discharges where they either were consumed by stream biota or settled into streambed sediment. The largest total nitrogen concentration upstream from WWTF discharges was $3.4 \mathrm{mg} / \mathrm{L}$ in a sample collected at site BL4 during the July 2003 synoptic survey; all other upstream samples contained less than $3.0 \mathrm{mg} / \mathrm{L}$ total nitrogen. Twenty-one of 73 base-flow samples contained total nitrogen concentrations that were less than USEPA ambient water-quality recommendation of $0.855 \mathrm{mg} / \mathrm{L}$ for level III ecoregion 40 (the central irregular plains) (U.S. Environmental Protection Agency, 2000b). Johnson County base-flow concentrations of total nitrogen were slightly larger than NAWQA median total nitrogen data 


\section{A. Big Bull Creek watershed}

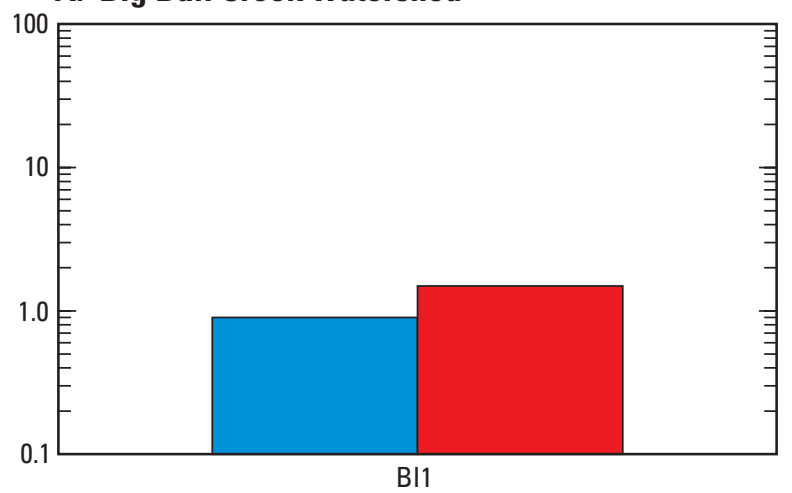

C. Brush Creek watershed

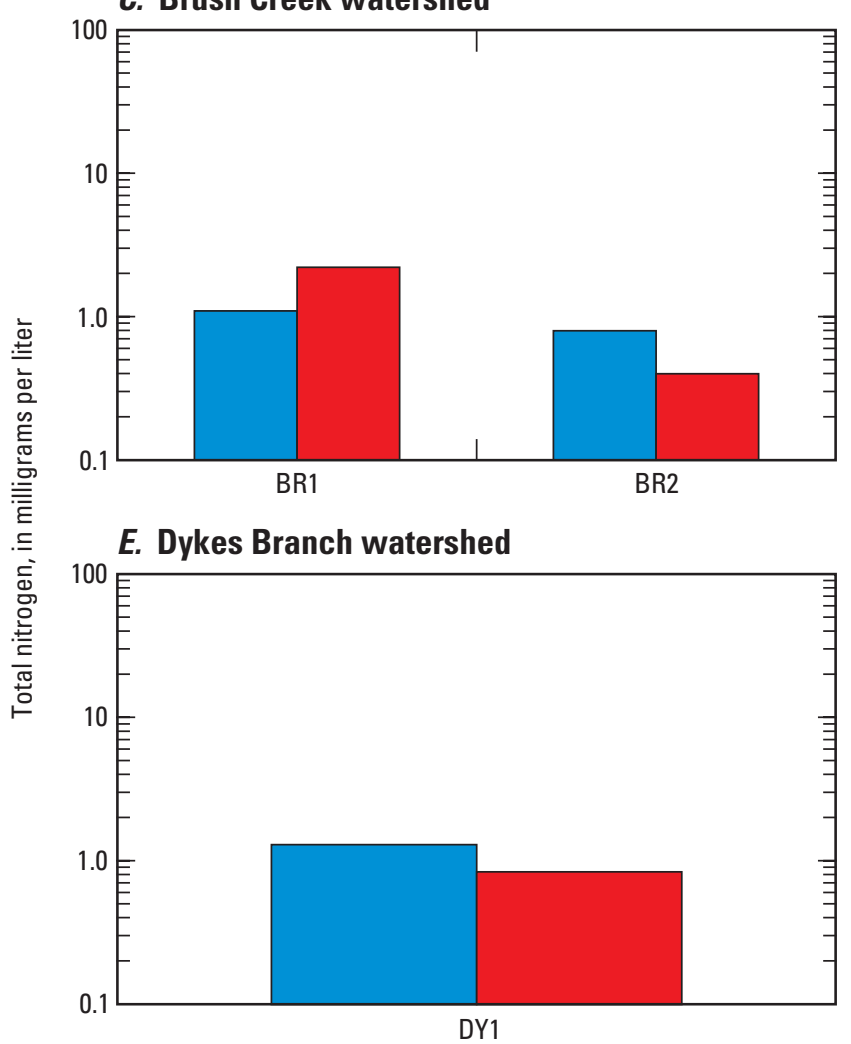

G. Kill Creek watershed

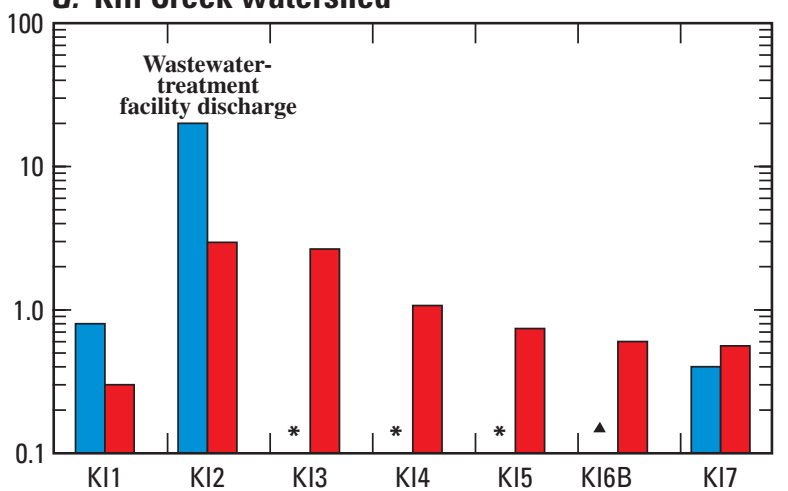

B. Blue River watershed

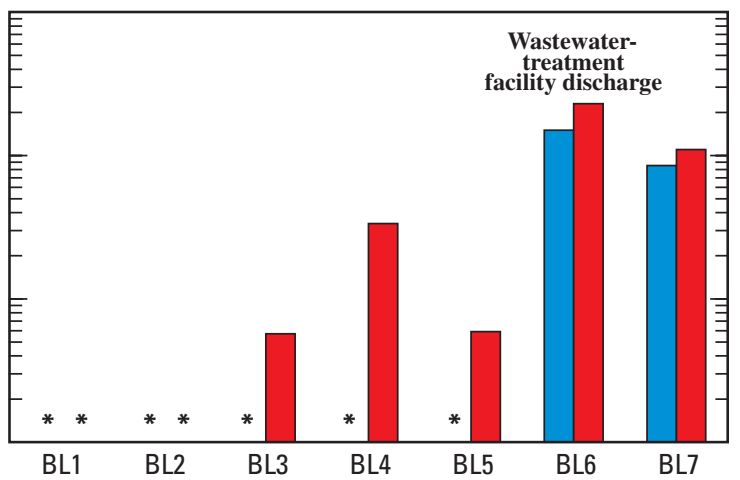

\section{Cedar Creek watershed}

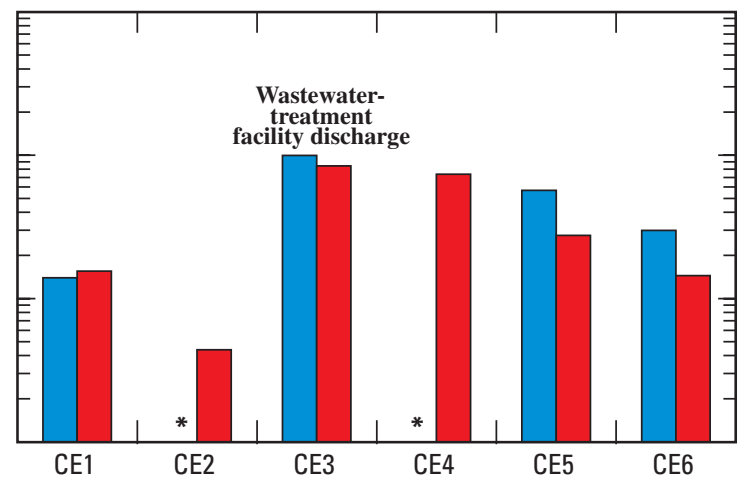

F. Indian Creek watershed

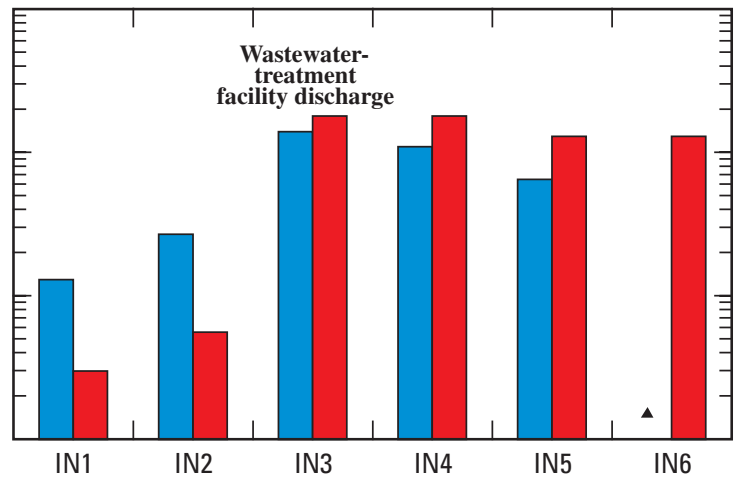

H. Little Bull Creek watershed

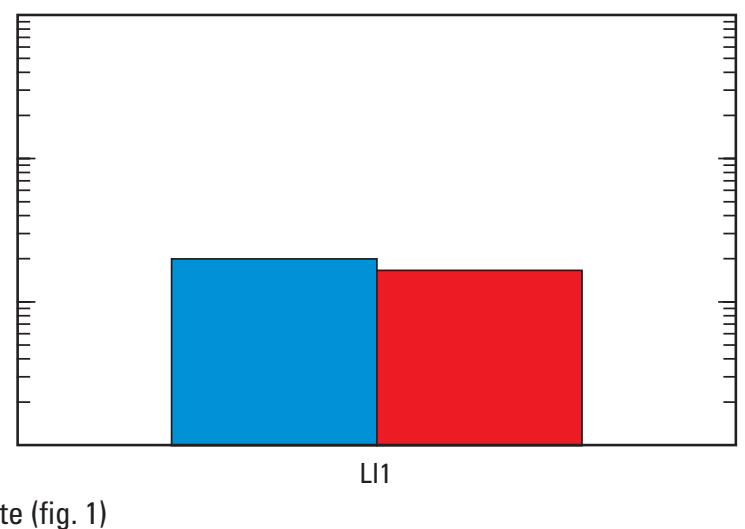

Figure 11. Total nitrogen concentrations in base-flow samples, November 4-7, 2002, and July 14-18, 2003. 

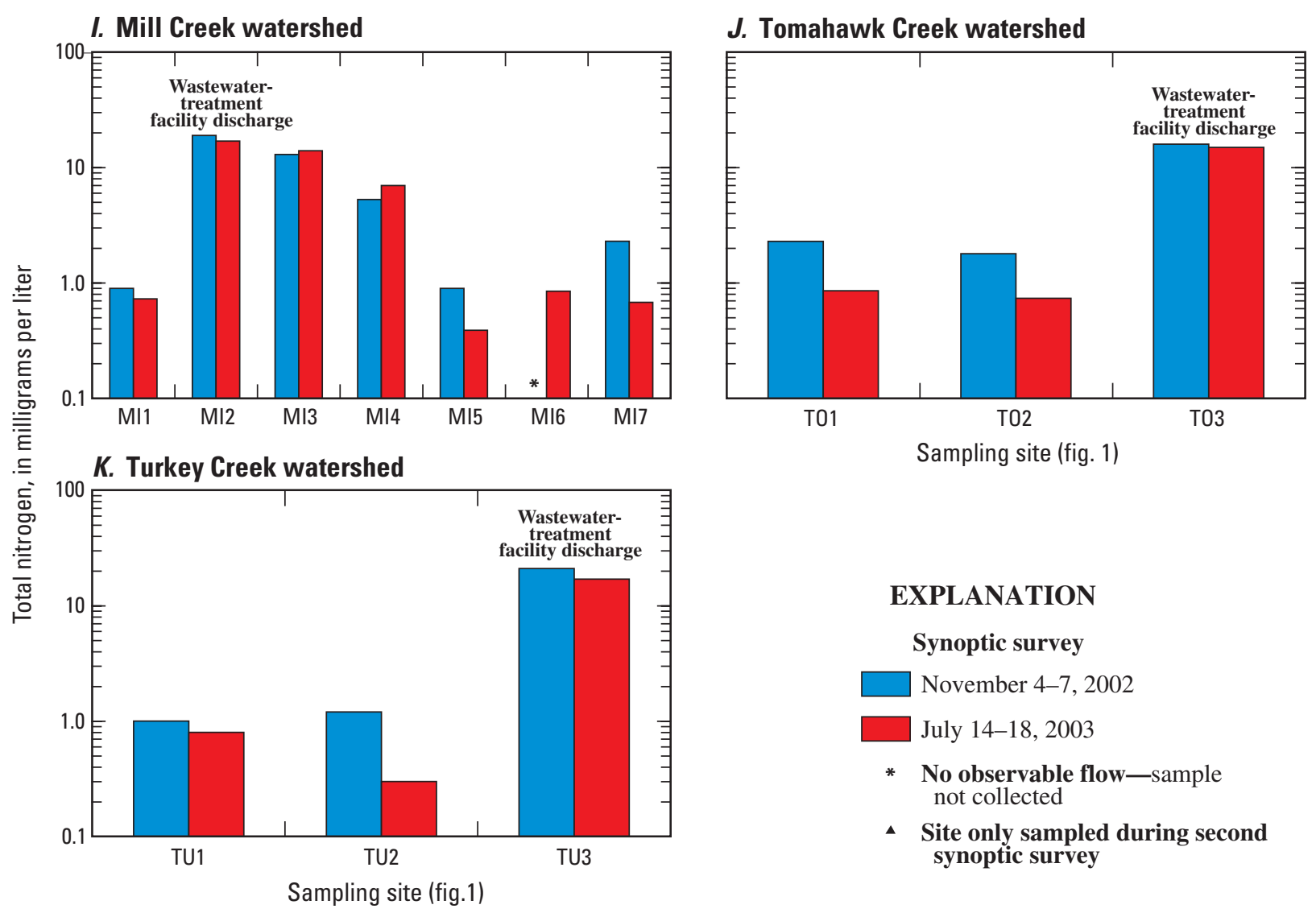

Figure 11. Total nitrogen concentrations in base-flow samples, November 4-7, 2002, and July 14-18, 2003.-Continued.

and less than maximum historical NAWQA values for either rural or urban watershed land use (table 13). Comparisons to USEPA ecoregion standards and USGS NAWQA data indicate that total nitrogen concentrations in samples from base-flow sites (mostly at and immediately downstream from WWTF discharges) were elevated compared to national conditions.

Although a Mann-Whitney test of independent groups indicated that total nitrogen concentrations were not significantly different ( $\mathrm{p}$ value $=0.09$ ) between all base- and stormflow samples, a Kendall's tau test found total nitrogen concentrations significantly positively correlated to streamflow conditions in samples collected from stormflow sites ( $\mathrm{p}$ value less than 0.01) (figs. 12 and 13). When all base-flow samples are combined, total nitrogen concentrations from WWTF discharges are similar to those in stormflow samples. However, total nitrogen concentrations from single stormflow sites generally increase with increasing streamflow. This is illustrated by maximum total nitrogen concentrations in base- $(13 \mathrm{mg} / \mathrm{L})$ and stormflow samples $(8.7 \mathrm{mg} / \mathrm{L})$ from site IN6, which show that samples from sites near WWTF discharges often had larger total nitrogen concentrations during base-flow conditions (fig. 12). There was a significant ( $\mathrm{p}$ value less than 0.01 ) difference between hourly load estimates of total nitrogen in baseand stormflow samples (fig. 13), indicating that nonpoint sources and (or) sanitary sewer overflows were likely the cause of significantly larger loads of nitrogen during the collection of stormflow samples. Median stormflow total nitrogen concentrations from stormflow sampling sites were larger than historical nationwide median total nitrogen values for rural or urban samples and did not exceed maximum values (table 14) (U.S. Geological Survey, 2004).

Analysis of nitrogen species in base-flow samples allow for further definition of nitrogen sources. Large ammonia concentrations in WWTF discharge are indicative of unoxidized sewage effluent. On the basis of onsite $\mathrm{pH}$ and watertemperature readings, base-flow samples at two of the three WWTF sites with trickling-filter secondary treatment processes (table 3)-sites TO3 (2.8 mg/L) and TU3 (2.5 mg/L) (table 13) - exceeded KDHE chronic aquatic-life criterion for total ammonia when early-life stages of fish are present (only applicable during July 2003 samples). Mean ammonia concentrations were smaller in samples from WWTF sites BL6 (0.62 mg/L), CE3 (0.08 mg/L), IN3 (0.25 mg/L), KI2 $(0.09 \mathrm{mg} / \mathrm{L})$, and MI2 $(0.08 \mathrm{mg} / \mathrm{L})$. Mean nitrite plus nitrate concentrations were larger in samples from activated-sludge WWTF sites BL6 (16 mg/L), CE3 (8.2 mg/L), IN3 (14 mg/L), and $\mathrm{KI} 2(11 \mathrm{mg} / \mathrm{L})$ as well as in samples from the trickling-filter WWTF site MI2 (16 mg/L). Treatment processes at these facilities showed improved oxidization of nutrient sources compared to the Tomahawk and Turkey Creek WWTFs (sites TO3 and TU3). 
Table 13. Results of analysis of nutrients in base-flow samples collected from selected Johnson County streams, northeastern Kansas, November 4-7, 2002, and July 14-18, 2003, level III ecoregion 40 criteria, and samples collected nationwide through the National Water-Quality Assessment Program (NAW0A), 1980-90.

[All concentrations are given in milligrams per liter; --, not determined; <, less than]

\begin{tabular}{|c|c|c|c|c|c|c|c|c|c|c|}
\hline \multirow[b]{2}{*}{ Sampling site (fig. 1) } & \multirow[b]{2}{*}{$\begin{array}{c}\text { Date of } \\
\text { sample } \\
\text { (month/day/ } \\
\text { year) }\end{array}$} & \multicolumn{8}{|c|}{ Nutrient concentrations } & \multirow[b]{2}{*}{$\begin{array}{l}\text { Phosphorus, } \\
\text { orthophos- } \\
\text { phate, as } \mathrm{P}\end{array}$} \\
\hline & & $\begin{array}{l}\text { Nitrogen, } \\
\text { total, as N }\end{array}$ & $\begin{array}{l}\text { Nitrogen, } \\
\text { nitrite, as N }\end{array}$ & $\begin{array}{c}\text { Nitrogen, } \\
\text { nitrite plus } \\
\text { nitrate, as } \mathrm{N}\end{array}$ & $\begin{array}{c}\text { Nitrogen, } \\
\text { ammonia, as } \\
\mathrm{N}\end{array}$ & $\begin{array}{c}\text { Nitrogen, } \\
\text { ammonia } \\
\text { plus } \\
\text { dissolved } \\
\text { organic } \\
\text { nitrogen, as } \\
\mathrm{N}\end{array}$ & $\begin{array}{l}\text { Nitrogen, } \\
\text { ammonia } \\
\text { plus total } \\
\text { organic } \\
\text { nitrogen, as } \\
\mathrm{N}\end{array}$ & $\begin{array}{c}\text { Phosphorus, } \\
\text { total }\end{array}$ & $\begin{array}{c}\text { Phosphorus, } \\
\text { dissolved }\end{array}$ & \\
\hline-- & -- & 0.855 & -- & 0.23 & -- & -- & 0.625 & 0.0925 & -- & -- \\
\hline \multicolumn{11}{|c|}{ NAWQA data (U.S. Geological Survey, 2004) } \\
\hline Rural median & -- & 1.6 & -- & .60 & 0.05 & -- & -- & .10 & -- & 0.04 \\
\hline Rural maximum & -- & 36 & -- & 36 & 35 & -- & -- & 9.1 & -- & 1.7 \\
\hline Urban median & -- & 1.7 & -- & .63 & .16 & -- & -- & .23 & -- & .02 \\
\hline Urban maximum & -- & 42 & -- & 12 & 21 & -- & -- & 8.3 & -- & 6.1 \\
\hline Johnson County median & -- & 1.8 & .06 & 1.5 & .08 & 0.4 & 6 & .40 & 0.24 & .45 \\
\hline $\begin{array}{l}\text { Johnson County } \\
\text { maximum } \\
\text { (sampling site, fig. 1) }\end{array}$ & -- & $\begin{array}{c}23 \\
\text { (site BL6) }\end{array}$ & $\begin{array}{r}.62 \\
\text { (site TU3) }\end{array}$ & $\begin{array}{c}20 \\
\text { (site BL6) }\end{array}$ & $\begin{array}{c}3.5 \\
\text { (site TU3) }\end{array}$ & $\begin{array}{c}5.5 \\
\text { (site TU3) }\end{array}$ & $\begin{array}{c}9.5 \\
\text { (site TU3) }\end{array}$ & $\begin{array}{c}4.7 \\
\text { (site MI2) }\end{array}$ & $\begin{array}{c}4.5 \\
\text { (site MI2) }\end{array}$ & $\begin{array}{c}4.5 \\
\text { (site MI2) }\end{array}$ \\
\hline \multicolumn{11}{|c|}{ Big Bull Creek watershed } \\
\hline BI1 & $11 / 4 / 02$ & .90 & $<.02$ & $<.10$ & $<.02$ & .4 & .9 & .34 & .25 & .22 \\
\hline & $7 / 14 / 03$ & 1.5 & .02 & .09 & .40 & 1.0 & 1.4 & .74 & .61 & .62 \\
\hline \multicolumn{11}{|c|}{ Blue River watershed } \\
\hline BL3 & $7 / 18 / 03$ & .57 & $<.02$ & .07 & .10 & .4 & .5 & .14 & .09 & .07 \\
\hline BL4 & 7/18/03 & 3.4 & .07 & 3.0 & .11 & .4 & .4 & .11 & .06 & $<.01$ \\
\hline BL5 & 7/18/03 & .59 & $<.02$ & .09 & .09 & .5 & .5 & .13 & .11 & .09 \\
\hline \multirow[t]{2}{*}{ BL6 } & $11 / 4 / 02$ & 15 & .04 & 13 & .04 & 1.2 & 1.8 & 2.7 & 2.5 & 2.5 \\
\hline & $7 / 18 / 03$ & 23 & .31 & 20 & 1.2 & 2.3 & 3.0 & 2.5 & 2.3 & 2.4 \\
\hline
\end{tabular}


Table 13. Results of analysis of nutrients in base-flow samples collected from selected Johnson County streams, northeastern Kansas, November 4-7, 2002, and July 14-18, 2003, level III ecoregion 40 criteria, and samples collected nationwide through the National Water-Quality Assessment Program (NAWOA), 1980-90.-Continued

[All concentrations are given in milligrams per liter; --, not determined; <, less than]

\begin{tabular}{|c|c|c|c|c|c|c|c|c|c|c|}
\hline \multirow[b]{2}{*}{ Sampling site (fig. 1) } & \multirow[b]{2}{*}{$\begin{array}{c}\text { Date of } \\
\text { sample } \\
\text { (month/day/ } \\
\text { year) }\end{array}$} & \multicolumn{8}{|c|}{ Nutrient concentrations } & \multirow[b]{2}{*}{$\begin{array}{c}\text { Phosphorus } \\
\text { orthophos- } \\
\text { phate, as P }\end{array}$} \\
\hline & & $\begin{array}{l}\text { Nitrogen, } \\
\text { total, as N }\end{array}$ & $\begin{array}{c}\text { Nitrogen, } \\
\text { nitrite, as N }\end{array}$ & $\begin{array}{c}\text { Nitrogen, } \\
\text { nitrite plus } \\
\text { nitrate, as N }\end{array}$ & $\begin{array}{c}\text { Nitrogen, } \\
\text { ammonia, as } \\
\mathrm{N}\end{array}$ & $\begin{array}{c}\text { Nitrogen, } \\
\text { ammonia } \\
\text { plus } \\
\text { dissolved } \\
\text { organic } \\
\text { nitrogen, as } \\
\mathrm{N}\end{array}$ & $\begin{array}{c}\text { Nitrogen, } \\
\text { ammonia } \\
\text { plus total } \\
\text { organic } \\
\text { nitrogen, as } \\
\text { N }\end{array}$ & $\begin{array}{c}\text { Phosphorus, } \\
\text { total }\end{array}$ & $\begin{array}{c}\text { Phosphorus, } \\
\text { dissolved }\end{array}$ & \\
\hline \multicolumn{11}{|c|}{ Brush Creek watershed } \\
\hline \multirow[t]{2}{*}{ BR1 } & $11 / 7 / 02$ & 1.1 & 0.03 & 0.40 & 0.04 & 0.5 & 0.7 & 0.13 & 0.09 & 0.09 \\
\hline & $7 / 15 / 03$ & 2.2 & .03 & .12 & .90 & 2.0 & 2.1 & .20 & .14 & .11 \\
\hline \multirow[t]{2}{*}{ BR2 } & $11 / 7 / 02$ & .80 & .02 & .40 & .02 & .3 & .4 & .08 & .05 & .04 \\
\hline & $7 / 15 / 03$ & .40 & $<.02$ & $<.05$ & .05 & .3 & .4 & .16 & .10 & .06 \\
\hline \multicolumn{11}{|c|}{ Cedar Creek watershed } \\
\hline \multirow[t]{2}{*}{ CE1 } & $11 / 4 / 02$ & 1.4 & $<.02$ & 1.0 & $<.02$ & .4 & .4 & .05 & .05 & .03 \\
\hline & $7 / 14 / 03$ & 1.6 & .06 & 1.1 & .20 & .5 & .5 & .07 & .03 & .01 \\
\hline CE2 & $7 / 14 / 03$ & .44 & $<.02$ & .14 & .08 & .2 & .3 & .11 & .05 & .04 \\
\hline \multirow[t]{2}{*}{ CE3 } & $11 / 4 / 02$ & 10 & .06 & 9.5 & .04 & .7 & .9 & 2.2 & 2.3 & 2.1 \\
\hline & $7 / 14 / 03$ & 8.5 & .40 & 6.8 & .12 & 1.0 & 1.7 & 2.7 & 2.6 & 2.4 \\
\hline CE4 & $7 / 14 / 03$ & 7.4 & .38 & 6.2 & .20 & .9 & 1.2 & 2.7 & 2.4 & 2.3 \\
\hline \multirow[t]{2}{*}{ CE5 } & $11 / 4 / 02$ & 5.7 & $<.02$ & 5.2 & .02 & .4 & .5 & .92 & .90 & .85 \\
\hline & $7 / 14 / 03$ & 2.8 & $<.02$ & 1.9 & .07 & .5 & .9 & 1.0 & .74 & .87 \\
\hline \multirow[t]{2}{*}{ CE6 } & $11 / 4 / 02$ & 3.0 & $<.02$ & 2.6 & $<.02$ & .4 & .4 & .77 & .76 & .72 \\
\hline & $7 / 14 / 03$ & 1.5 & .02 & .85 & .07 & .4 & .6 & .54 & .43 & .45 \\
\hline \multicolumn{11}{|c|}{ Dykes Branch watershed } \\
\hline \multirow[t]{2}{*}{ DY1 } & $11 / 7 / 02$ & 1.3 & .05 & .50 & .10 & .4 & .8 & .17 & .13 & .12 \\
\hline & $7 / 17 / 03$ & .84 & $<.02$ & .54 & .04 & .4 & .3 & .24 & .22 & .21 \\
\hline \multicolumn{11}{|c|}{ Indian Creek watershed } \\
\hline \multirow[t]{2}{*}{ IN1 } & $11 / 6 / 02$ & 1.3 & .03 & .80 & $<.02$ & .3 & .5 & .05 & $<.01$ & $<.01$ \\
\hline & $7 / 17 / 03$ & .30 & $<.02$ & $<.05$ & .02 & .2 & .3 & .04 & .02 & $<.01$ \\
\hline
\end{tabular}


Table 13. Results of analysis of nutrients in base-flow samples collected from selected Johnson County streams, northeastern Kansas, November 4-7, 2002, and July 14-18, 2003, level III ecoregion 40 criteria, and samples collected nationwide through the National Water-Quality Assessment Program (NAWQA), 1980-90.-Continued

[All concentrations are given in milligrams per liter; --, not determined; <, less than]

\begin{tabular}{|c|c|c|c|c|c|c|c|c|c|c|}
\hline \multirow[b]{2}{*}{ Sampling site (fig. 1) } & \multicolumn{10}{|c|}{ Nutrient concentrations } \\
\hline & $\begin{array}{c}\text { Date of } \\
\text { sample } \\
\text { (month/day/ } \\
\text { year) }\end{array}$ & $\begin{array}{l}\text { Nitrogen, } \\
\text { total, as N }\end{array}$ & $\begin{array}{c}\text { Nitrogen, } \\
\text { nitrite, as N }\end{array}$ & $\begin{array}{l}\text { Nitrogen, } \\
\text { nitrite plus } \\
\text { nitrate, as } \mathrm{N}\end{array}$ & $\begin{array}{c}\text { Nitrogen, } \\
\text { ammonia, as } \\
\mathrm{N}\end{array}$ & $\begin{array}{l}\text { Nitrogen, } \\
\text { ammonia } \\
\text { plus } \\
\text { dissolved } \\
\text { organic } \\
\text { nitrogen, as } \\
\text { N }\end{array}$ & $\begin{array}{l}\text { Nitrogen, } \\
\text { ammonia } \\
\text { plus total } \\
\text { organic } \\
\text { nitrogen, as } \\
\mathrm{N}\end{array}$ & $\begin{array}{l}\text { Phosphorus, } \\
\text { total }\end{array}$ & $\begin{array}{l}\text { Phosphorus, } \\
\text { dissolved }\end{array}$ & $\begin{array}{c}\text { Phosphorus, } \\
\text { orthophos- } \\
\text { phate, as P }\end{array}$ \\
\hline \multicolumn{11}{|c|}{ Indian Creek watershed-Continued } \\
\hline \multirow[t]{2}{*}{ IN2 } & $11 / 6 / 02$ & 2.7 & 0.02 & 1.3 & $<0.02$ & 0.4 & 0.7 & 0.06 & 0.03 & 0.03 \\
\hline & $7 / 17 / 03$ & .56 & $<.02$ & .16 & .07 & .2 & .4 & .04 & .03 & .02 \\
\hline \multirow[t]{2}{*}{ IN3 } & $11 / 6 / 02$ & 14 & .08 & 12 & .30 & 1.0 & 2.0 & 4.0 & 3.7 & 3.5 \\
\hline & $7 / 17 / 03$ & 18 & .22 & 17 & .20 & 1.1 & 1.3 & 3.8 & 3.8 & 3.8 \\
\hline \multirow[t]{2}{*}{ IN4 } & $11 / 6 / 02$ & 11 & .06 & 9.2 & .20 & .8 & 1.4 & 3.2 & 2.5 & 2.5 \\
\hline & $7 / 17 / 03$ & 18 & .14 & 17 & .20 & 1.1 & 1.3 & 3.6 & 3.4 & 3.5 \\
\hline \multirow[t]{2}{*}{ IN5 } & $11 / 6 / 02$ & 6.5 & .08 & 4.7 & .50 & 1.2 & 1.8 & 1.5 & 1.3 & 1.4 \\
\hline & $7 / 17 / 03$ & 13 & .14 & 11 & .60 & 2.1 & 2.2 & 3.1 & 3.0 & 2.9 \\
\hline IN6 & $7 / 17 / 03$ & 13 & .26 & 11 & .50 & 1.3 & 1.8 & 3.0 & 2.9 & 2.8 \\
\hline \multicolumn{11}{|c|}{ Kill Creek watershed } \\
\hline \multirow[t]{2}{*}{ KI1 } & $11 / 4 / 02$ & .80 & $<.02$ & .60 & $<.02$ & .2 & .2 & .05 & .03 & .02 \\
\hline & $7 / 15 / 03$ & .30 & $<.02$ & $<.05$ & .03 & .2 & .3 & .07 & .02 & $<.01$ \\
\hline \multirow[t]{2}{*}{ KI2 } & $11 / 4 / 02$ & 20 & .06 & 19 & .09 & 1.1 & 1.2 & 3.7 & 3.6 & 3.7 \\
\hline & $7 / 15 / 03$ & 3.0 & $<.02$ & 2.1 & .09 & .9 & .9 & 3.2 & 3.2 & 3.3 \\
\hline KI3 & $7 / 15 / 03$ & 2.7 & .05 & 1.8 & .10 & .8 & .9 & 2.8 & 2.7 & .53 \\
\hline KI4 & $7 / 15 / 03$ & 1.1 & $<.02$ & .47 & .06 & .4 & 6 & .91 & .86 & .88 \\
\hline KI5 & $7 / 15 / 03$ & .74 & $<.02$ & .24 & .05 & .3 & .5 & .72 & .63 & .62 \\
\hline KI6 & $7 / 15 / 03$ & .60 & $<.02$ & $<.05$ & .02 & .3 & .6 & .17 & .11 & .08 \\
\hline \multirow[t]{2}{*}{ KI7 } & $11 / 4 / 02$ & .40 & $<.02$ & .10 & $<.02$ & .2 & .3 & .10 & .07 & .05 \\
\hline & $7 / 15 / 03$ & .56 & $<.02$ & .16 & .06 & .2 & .4 & .14 & .10 & .09 \\
\hline \multicolumn{11}{|c|}{ Little Bull Creek watershed } \\
\hline \multirow[t]{2}{*}{ LI1 } & $11 / 4 / 02$ & 2.0 & .02 & 1.1 & $<.02$ & 6 & .9 & .18 & .12 & .10 \\
\hline & $7 / 14 / 03$ & 1.7 & .03 & .56 & .10 & .6 & 1.1 & .22 & .08 & .01 \\
\hline
\end{tabular}


Table 13. Results of analysis of nutrients in base-flow samples collected from selected Johnson County streams, northeastern Kansas, November 4-7, 2002, and July 14-18, 2003, level III ecoregion 40 criteria, and samples collected nationwide through the National Water-Quality Assessment Program (NAWQA), 1980-90.-Continued

[All concentrations are given in milligrams per liter; --, not determined; <, less than]

\begin{tabular}{|c|c|c|c|c|c|c|c|c|c|c|}
\hline \multirow[b]{2}{*}{ Sampling site (fig. 1) } & \multirow[b]{2}{*}{$\begin{array}{c}\text { Date of } \\
\text { sample } \\
\text { (month/day/ } \\
\text { year) }\end{array}$} & \multicolumn{8}{|c|}{ Nutrient concentrations } & \multirow[b]{2}{*}{$\begin{array}{c}\text { Phosphorus, } \\
\text { orthophos- } \\
\text { phate, as P }\end{array}$} \\
\hline & & $\begin{array}{l}\text { Nitrogen, } \\
\text { total, as N }\end{array}$ & $\begin{array}{c}\text { Nitrogen, } \\
\text { nitrite, as N }\end{array}$ & $\begin{array}{c}\text { Nitrogen, } \\
\text { nitrite plus } \\
\text { nitrate, as } \mathrm{N}\end{array}$ & $\begin{array}{c}\text { Nitrogen, } \\
\text { ammonia, as } \\
\mathrm{N}\end{array}$ & $\begin{array}{l}\text { Nitrogen, } \\
\text { ammonia } \\
\text { plus } \\
\text { dissolved } \\
\text { organic } \\
\text { nitrogen, as } \\
\mathrm{N}\end{array}$ & $\begin{array}{l}\text { Nitrogen, } \\
\text { ammonia } \\
\text { plus total } \\
\text { organic } \\
\text { nitrogen, as } \\
\text { N }\end{array}$ & $\begin{array}{c}\text { Phosphorus, } \\
\text { total }\end{array}$ & $\begin{array}{c}\text { Phosphorus, } \\
\text { dissolved }\end{array}$ & \\
\hline \multicolumn{11}{|c|}{ Mill Creek watershed } \\
\hline \multirow[t]{2}{*}{ MI1 } & $11 / 4 / 02$ & 0.90 & 0.03 & 0.40 & 0.11 & 0.4 & 0.5 & 0.17 & 0.10 & 0.09 \\
\hline & $7 / 16 / 03$ & .73 & $<.02$ & .23 & .06 & .3 & .5 & .09 & .07 & .06 \\
\hline \multirow[t]{2}{*}{ MI2 } & $11 / 4 / 02$ & 19 & .06 & 17 & .07 & 1.8 & 2.1 & 4.6 & 4.3 & 4.5 \\
\hline & $7 / 16 / 03$ & 17 & .16 & 15 & .10 & .7 & 2.1 & 4.7 & 4.5 & 4.4 \\
\hline \multirow[t]{2}{*}{ MI3 } & $11 / 4 / 02$ & 13 & .06 & 11 & .08 & 1.4 & 1.5 & 3.2 & 3.0 & 3.2 \\
\hline & $7 / 16 / 03$ & 14 & .13 & 12 & .20 & 1.1 & 1.7 & 4.2 & 4.0 & 4.0 \\
\hline \multirow[t]{2}{*}{ MI4 } & $11 / 4 / 02$ & 5.3 & .02 & 4.8 & $<.02$ & .4 & .5 & 1.0 & 1.0 & 1.0 \\
\hline & $7 / 16 / 03$ & 7.0 & .05 & 6.5 & .04 & .4 & .5 & 2.2 & 1.2 & 1.1 \\
\hline \multirow[t]{2}{*}{ MI5 } & $11 / 4 / 02$ & .90 & $<.02$ & .70 & $<.02$ & .2 & .2 & .06 & .06 & .04 \\
\hline & $7 / 16 / 03$ & .39 & $<.02$ & .19 & .05 & .3 & .2 & .10 & .07 & .06 \\
\hline MI6 & $7 / 16 / 03$ & .85 & $<.02$ & .45 & .08 & .3 & .4 & 4.5 & .03 & .02 \\
\hline \multirow[t]{2}{*}{ MI7 } & $11 / 4 / 02$ & 2.3 & $<.02$ & 1.9 & $<.02$ & .2 & .4 & .40 & .39 & .35 \\
\hline & $7 / 16 / 03$ & .68 & $<.02$ & .28 & .03 & .3 & .4 & .28 & .19 & .18 \\
\hline \multicolumn{11}{|c|}{ Tomahawk Creek watershed } \\
\hline \multirow[t]{2}{*}{ TO1 } & $11 / 6 / 02$ & 2.3 & $<.02$ & 1.7 & .02 & .4 & .6 & .05 & .04 & .04 \\
\hline & $7 / 17 / 03$ & .86 & $<.02$ & .46 & .03 & .3 & .4 & .08 & .03 & $<.01$ \\
\hline \multirow[t]{2}{*}{ TO2 } & $11 / 6 / 02$ & 1.8 & .02 & 1.2 & .02 & .4 & .6 & .05 & .03 & .03 \\
\hline & $7 / 17 / 03$ & .74 & $<.02$ & .34 & .07 & .4 & .4 & .05 & .03 & .02 \\
\hline \multirow[t]{2}{*}{ TO3 } & $11 / 6 / 02$ & 16 & .19 & 9.6 & 2.4 & 4.7 & 6.2 & 4.2 & 3.3 & 3.4 \\
\hline & $7 / 17 / 03$ & 15 & .22 & 9.1 & 2.8 & 5.4 & 6.3 & 4.5 & 4.3 & 4.3 \\
\hline \multicolumn{11}{|c|}{ Turkey Creek watershed } \\
\hline \multirow[t]{2}{*}{ TU1 } & $11 / 7 / 02$ & 1.0 & .02 & .40 & .03 & .2 & 6 & .04 & .02 & .02 \\
\hline & $7 / 16 / 03$ & .80 & $<.02$ & $<.05$ & .03 & .6 & .8 & .08 & .01 & $<.01$ \\
\hline \multirow[t]{2}{*}{ TU2 } & $11 / 7 / 02$ & 1.2 & .02 & .60 & .02 & 6 & .6 & .02 & .01 & .01 \\
\hline & $7 / 16 / 03$ & .30 & $<.02$ & $<.05$ & .03 & .2 & .3 & .11 & .04 & .03 \\
\hline \multirow[t]{2}{*}{ TU3 } & $11 / 7 / 02$ & 21 & .62 & 11 & 3.5 & 5.5 & 9.5 & 3.8 & 3.4 & 3.2 \\
\hline & $7 / 16 / 03$ & 17 & .58 & 11 & 2.5 & 4.6 & 5.6 & 4.1 & 3.8 & 3.6 \\
\hline
\end{tabular}




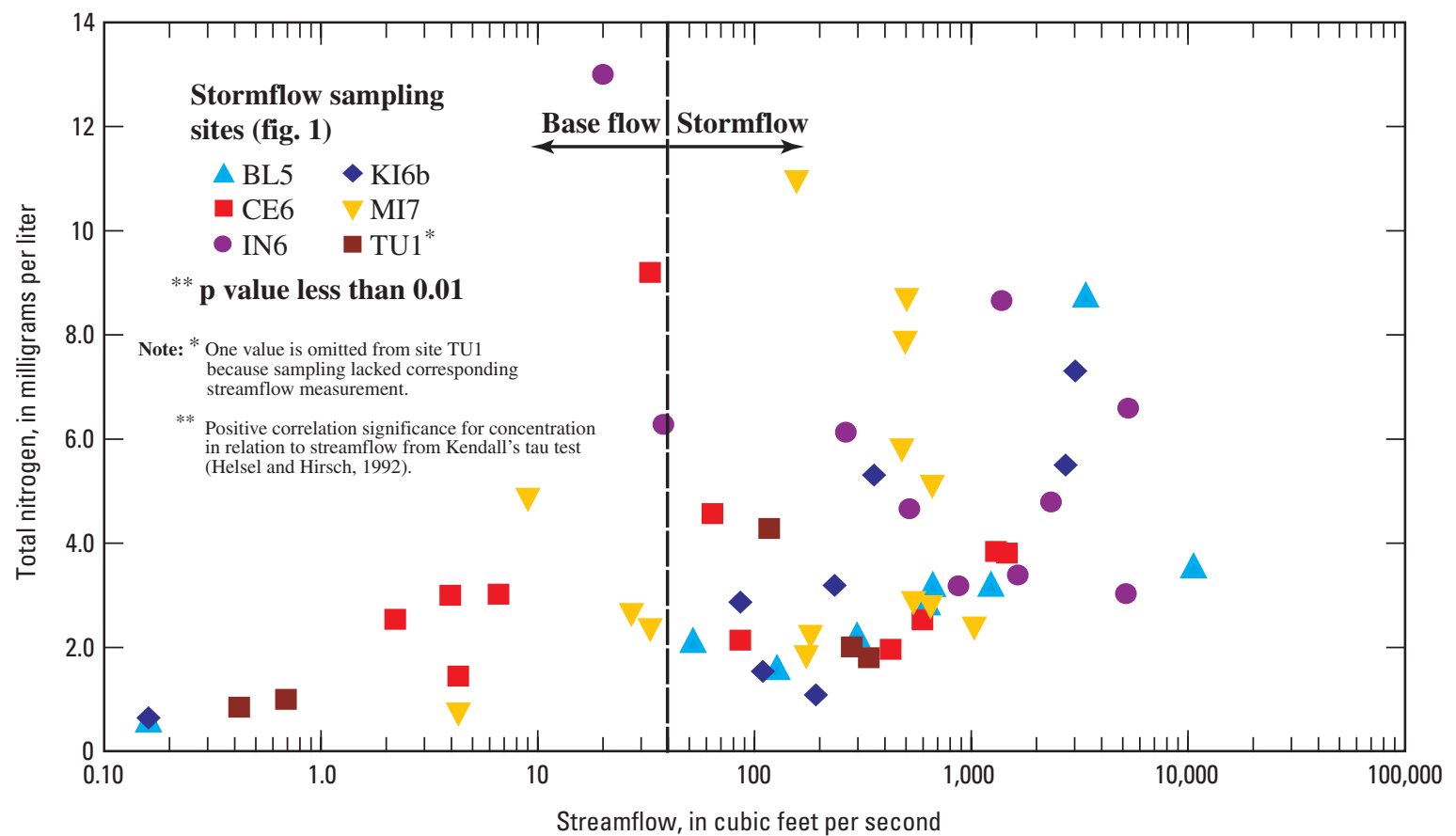

Figure 12. Total nitrogen concentrations in base- and stormflow samples collected at stormflow sampling sites, October 2002-June 2004.
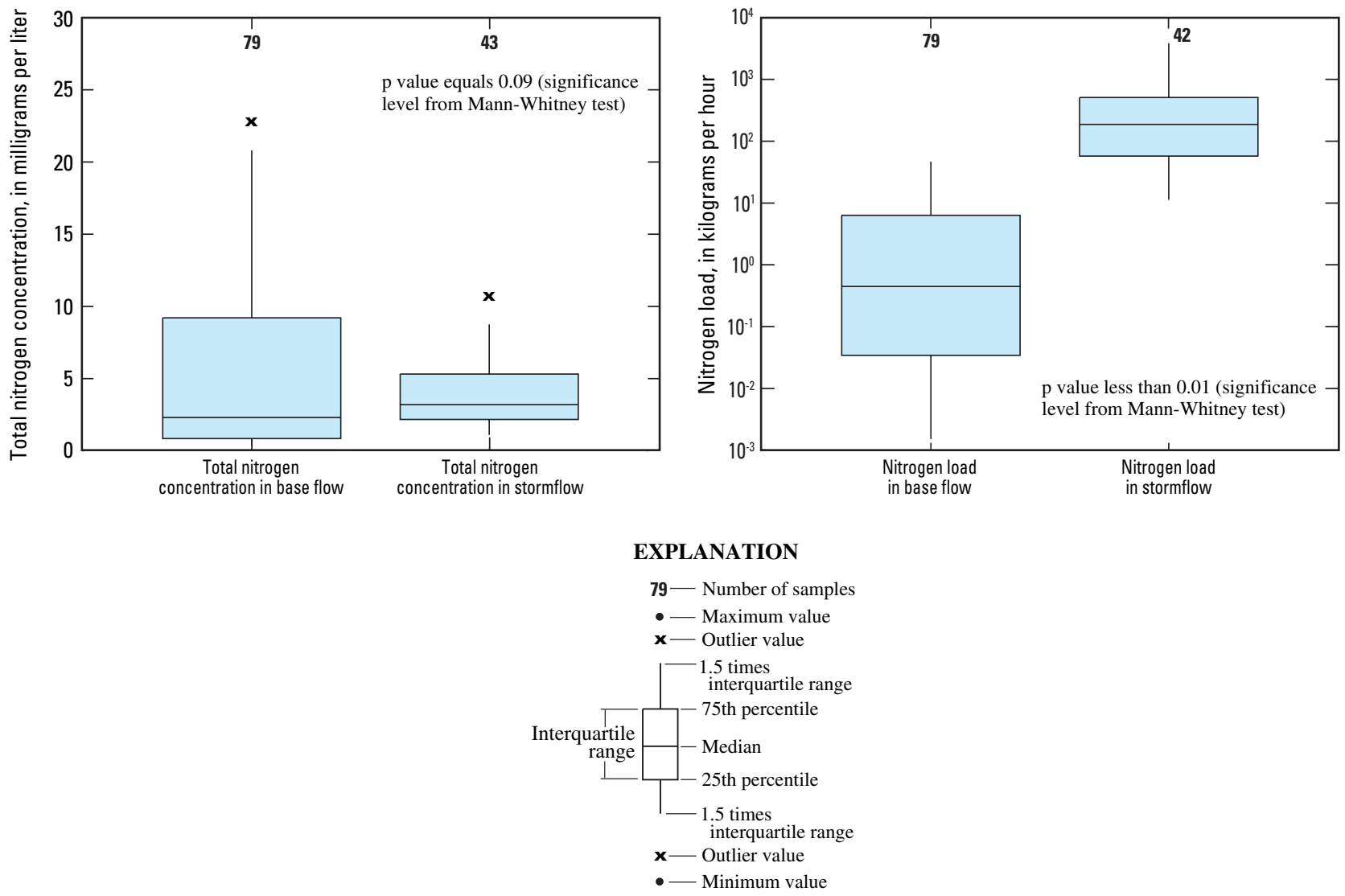

Figure 13. Total nitrogen concentrations and estimated loads in base- and stormflow samples, 0ctober 2002-June 2004. 
Table 14. Statistical summary of the results of analysis of nutrients collected from Johnson County stormflow sampling sites, northeastern Kansas, October 2002-June 2004, and samples collected nationwide through the National Water-Quality Assessment Program (NAWQA), 1980-90.

[NAWQA data from U.S. Geological Survey (2004). --, not determined; <, less than]

\begin{tabular}{|c|c|c|c|c|}
\hline \multirow{2}{*}{ Sampling site identifier (fig. 1) } & \multirow{2}{*}{$\begin{array}{c}\text { Number of } \\
\text { samples }\end{array}$} & \multicolumn{3}{|c|}{ Concentrations (milligrams per liter) } \\
\hline & & Minimum & Median & Maximum \\
\hline \multicolumn{5}{|c|}{ Total nitrogen } \\
\hline BL5 & 9 & 0.59 & 2.8 & 8.8 \\
\hline CE6 & 11 & 1.5 & 3.0 & 9.2 \\
\hline IN6 & 10 & 3.0 & 5.5 & 13 \\
\hline KI6b & 8 & .65 & 3.0 & 7.3 \\
\hline MI7 & 14 & .68 & 2.8 & 11 \\
\hline TU1 & 6 & .85 & 1.8 & 4.3 \\
\hline NAWQA rural samples & 4,036 & -- & 1.6 & 36 \\
\hline NAWQA urban samples & 1,985 & -- & 1.7 & 42 \\
\hline \multicolumn{5}{|c|}{ Nitrogen, nitrite } \\
\hline BL5 & 9 & $<.02$ & .02 & .03 \\
\hline CE6 & 11 & $<.02$ & .02 & .07 \\
\hline IN6 & 10 & .02 & .09 & .3 \\
\hline KI6b & 8 & $<.02$ & .02 & .03 \\
\hline MI7 & 14 & $<.02$ & $<.02$ & .1 \\
\hline TU1 & 6 & $<.02$ & .02 & .05 \\
\hline \multicolumn{5}{|c|}{ Nitrogen, nitrite plus nitrate } \\
\hline BL5 & 9 & .09 & .80 & 1.2 \\
\hline CE6 & 11 & .85 & 1.2 & 7.9 \\
\hline IN6 & 10 & .49 & 1.8 & 11 \\
\hline KI6b & 8 & .05 & .90 & 1.7 \\
\hline MI7 & 14 & .28 & 1.6 & 9.4 \\
\hline TU1 & 6 & .05 & .46 & .68 \\
\hline NAWQA rural samples & 6,894 & -- & .60 & 36 \\
\hline NAWQA urban samples & 3,234 & -- & .60 & 12 \\
\hline \multicolumn{5}{|c|}{ Nitrogen, ammonia } \\
\hline BL5 & 8 & .04 & .08 & .28 \\
\hline CE6 & 14 & $<.02$ & .07 & .12 \\
\hline IN6 & 10 & .07 & .30 & .80 \\
\hline KI6b & 8 & .05 & .86 & 1.7 \\
\hline MI7 & 14 & $<.02$ & .07 & .34 \\
\hline TU1 & 7 & .03 & .18 & .31 \\
\hline NAWQA rural samples & 5,895 & -- & .05 & 35 \\
\hline NAWQA urban samples & 3,356 & -- & .20 & 21 \\
\hline \multicolumn{5}{|c|}{ Nitrogen, ammonia plus dissolved organic nitrogen } \\
\hline BL5 & 9 & .07 & .50 & 1.3 \\
\hline CE6 & 11 & .40 & .69 & 1.0 \\
\hline IN6 & 10 & .60 & 1.2 & 1.5 \\
\hline KI6b & 8 & .30 & .80 & 1.3 \\
\hline MI7 & 14 & .20 & .60 & 1.0 \\
\hline TU1 & 6 & .20 & .60 & 1.1 \\
\hline
\end{tabular}


Table 14. Statistical summary of the results of analysis of nutrients collected from Johnson County stormflow sampling sites, northeastern Kansas, October 2002-June 2004, and samples collected nationwide through the National Water-Quality Assessment Program (NAWQA), 1980-90.-Continued

[NAWQA data from U.S. Geological Survey (2004). --, not determined; <, less than]

\begin{tabular}{|c|c|c|c|c|}
\hline \multirow{2}{*}{ Sampling site identifier (fig. 1) } & \multirow{2}{*}{$\begin{array}{c}\text { Number of } \\
\text { samples }\end{array}$} & \multicolumn{3}{|c|}{ Concentrations (milligrams per liter) } \\
\hline & & Minimum & Median & Maximum \\
\hline \multicolumn{5}{|c|}{ Nitrogen, ammonia plus total organic nitrogen } \\
\hline BL5 & 9 & 0.50 & 1.9 & 7.6 \\
\hline CE6 & 11 & .40 & 1.1 & 2.8 \\
\hline IN6 & 10 & 1.5 & 2.2 & 8.0 \\
\hline KI6b & 8 & 60 & 1.7 & 6.2 \\
\hline MI7 & 14 & .40 & 1.3 & 5.8 \\
\hline TU1 & 6 & 60 & 1.3 & 3.3 \\
\hline \multicolumn{5}{|c|}{ Phosphorus, total } \\
\hline BL5 & 9 & .13 & .53 & 2.5 \\
\hline CE6 & 11 & .31 & .66 & 2.4 \\
\hline IN6 & 10 & .62 & 1.2 & 3.0 \\
\hline KI6b & 8 & .17 & .50 & 2.1 \\
\hline MI7 & 14 & .28 & .69 & 2.4 \\
\hline TU1 & 6 & .04 & .48 & .84 \\
\hline NAWQA rural samples & 6,542 & -- & .10 & 9.1 \\
\hline NAWQA urban samples & 2,954 & -- & .23 & 8.3 \\
\hline \multicolumn{5}{|c|}{ Phosphorus, dissolved } \\
\hline BL5 & 9 & .06 & .10 & .16 \\
\hline CE6 & 11 & .06 & .43 & 2.3 \\
\hline IN6 & 10 & .07 & .47 & 2.9 \\
\hline KI6b & 8 & .06 & .12 & .27 \\
\hline MI7 & 14 & .09 & .23 & 1.5 \\
\hline TU1 & 6 & .01 & .08 & .11 \\
\hline \multicolumn{5}{|c|}{ Phosphorus, orthophosphate } \\
\hline BL5 & 9 & .04 & .08 & .12 \\
\hline CE6 & 11 & .05 & .45 & 2.2 \\
\hline IN6 & 10 & .06 & .31 & 2.8 \\
\hline KI6b & 8 & .04 & .09 & .27 \\
\hline MI7 & 14 & .02 & .18 & 1.5 \\
\hline TU1 & 6 & .01 & .06 & .10 \\
\hline NAWQA rural samples & 3,446 & -- & .04 & 1.7 \\
\hline NAWQA urban samples & 882 & -- & .02 & 6.1 \\
\hline
\end{tabular}


Nitrogen species in stormflow samples varied among sites and streamflow conditions (table 14, fig. 14). A Kendall's tau correlation test on dissolved nitrogen species showed a significant ( $p$ value less than 0.01 ) negative correlation with streamflow for all combined values at stormflow sites (fig. 14). This test shows that organic and particulate nitrogen likely comprise a larger portion of nonpoint sources, whereas dissolved nitrogen species comprise a larger portion of point-source contamination. However, there was considerable variability, often in samples from the same sites during similar flow conditions.

\section{Phosphorus}

Phosphorus occurs in the environment as both dissolved and particulate phosphorus. Dissolved phosphorus consists of inorganic orthophosphorus (bioavailable phosphorus) and organic phosphorus, which is a combination of phosphorus excreted by organisms and macromolecular colloidal phosphorus. Total phosphorus incorporates dissolved and particulate phosphorus. In most surface water, phosphorus concentrations limit aquatic plant and algae growth but in excess may be a primary cause of stream eutrophication. Wastewater discharge and runoff from both urban and agricultural land uses are the primary sources of phosphorus in surface water (Hem, 1992).

WWTFs were the primary source of total phosphorus in Johnson County streams during base-flow conditions (fig. 15, table 13). The largest mean total phosphorus concentrations were in samples from WWTF sites BL6 $(2.6 \mathrm{mg} / \mathrm{L})$, CE3 (2.4 mg/L), IN3 (3.9 mg/L), KI2 (3.4 mg/L), MI2 (4.6 mg/L), TO3 (4.4 mg/L), and TU3 (4.0 mg/L). The base-flow sample from site MI6 also had one of the largest total phosphorus concentrations $(4.5 \mathrm{mg} / \mathrm{L})$. However, because 99 percent of total phosphorus in this sample was associated with particulate material, this value likely was due to streambed sediment that was suspended by the sampling crew or by a disturbance upstream.

Total phosphorus concentrations decreased downstream from WWTF discharges as total phosphorus either was assimilated by aquatic life or adsorbed to streambed sediment. Eighteen of 73 base-flow samples (table 13) had a total phosphorus concentration that was less than the USEPA ambient waterquality recommendation of $0.0925 \mathrm{mg} / \mathrm{L}$ for level III ecoregion 40 (U.S. Environmental Protection Agency, 2000b). The median total phosphorus concentration of all base-flow samples was about two to four times larger than historical NAWQA median total phosphorus concentrations, and smaller than maximum NAWQA concentrations for either rural or urban samples (table 13) (U.S. Geological Survey, 2004). Comparisons to USEPA ecoregion standards and USGS NAWQA data indicate that total phosphorus concentrations at base-flow sites (mostly at and immediately downstream from WWTF discharges) were elevated compared to national conditions.

Although total phosphorus concentrations were not significantly different ( $\mathrm{p}$ value $=0.36$ ) between all base- and stormflow samples, they were shown to significantly increase during larger streamflow conditions in samples from stormflow sites
( $p$ value less than 0.01) (figs. 13 and 16). When all base-flow samples are combined, concentrations from WWTF discharges are similar to those in stormflow samples. However, total phosphorus concentrations from single stormflow sites generally increase with increasing streamflow. This is illustrated by maximum total phosphorus concentrations in base- $(3.0 \mathrm{mg} / \mathrm{L})$ and stormflow samples $(2.7 \mathrm{mg} / \mathrm{L})$ from site IN6, which show that samples from sites near WWTF discharges can have larger total phosphorus concentrations during base-flow than during stormflow conditions (fig. 16). There was a significant ( $p$ value less than 0.01) difference between hourly load estimates of total phosphorus in base- and stormflow samples (fig. 17). This indicates that nonpoint sources and (or) sanitary sewer overflows likely caused significantly larger loads of phosphorus during stormflow samples. Median total phosphorus concentrations for these six sites ranged from about 5 to 13 times the historical NAWQA median total phosphorus values and were less than historical maximum NAWQA values for rural or urban samples (table 14) (U.S. Geological Survey, 2004).

Concentrations of orthophosphorus represented most of the total phosphorus concentration in samples of Johnson County wastewater discharges. Orthophosphorus represented more than 80 percent of total phosphorus in all samples of WWTF discharge and was more than 90 percent of total phosphorus in 10 of 16 WWTF discharge samples. Large concentrations of orthophosphorus indicate that almost all discharged phosphorus is bioavailable and likely utilized by aquatic plants and algae downstream from WWTF discharges.

A Kendall's tau correlation test on dissolved phosphorus species showed a significant ( $\mathrm{p}$ value less than 0.01 ) negative correlation with streamflow for all combined values at stormflow sampling sites (fig. 14B). This test shows that organic and particulate phosphorus likely are contributed by nonpoint sources, whereas dissolved phosphorus species comprise a larger portion of point-source contamination. However, there was considerable variability, often in samples from the same sites during similar flow conditions.

\section{Bacteria}

Fecal coliform, Escherichia coli (E. coli), and enterococci bacteria were analyzed in all stream-water samples. These bacteria are "indicator bacteria," which are used to represent a potential for pathogenicity of surface water. Although these bacteria generally are not toxic themselves, they are used to represent all viruses, protozoa, and pathogenic bacteria that may cause illnesses associated with water contact and ingestion (U.S. Environmental Protection Agency, 2004b).

Fecal coliform bacteria were the original indicator bacteria standard used nationally and in Kansas and are still used in Johnson County and around the nation to regulate municipal wastewater-treatment facilities (E. Hack, Johnson County Wastewater and Public Works, written commun., 2003). National Pollutant Discharge Elimination System (NPDES) wastewater permits in Johnson County mandate a maximum 


\section{A. Dissolved nitrogen}

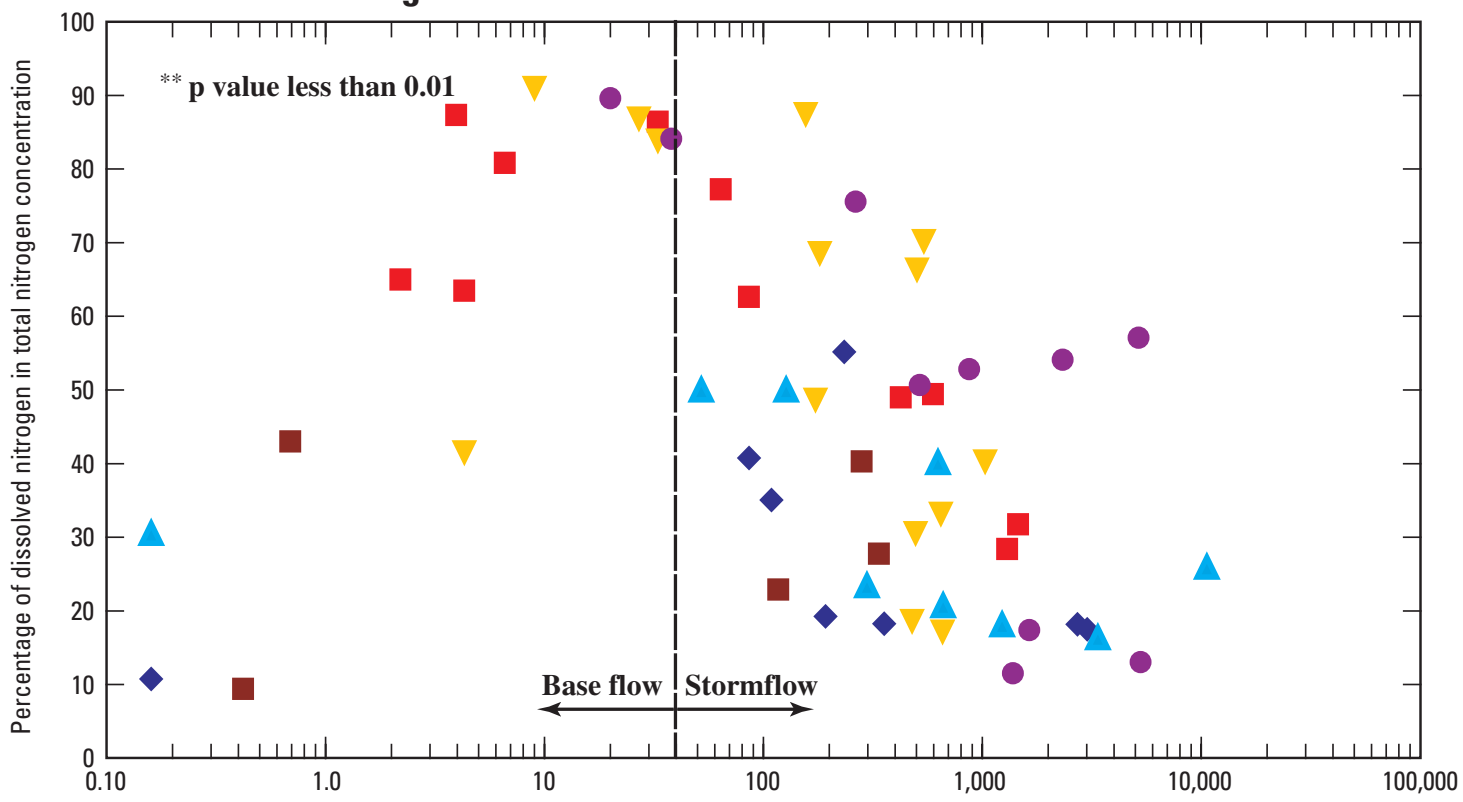

\section{B. Dissolved phosphorus}

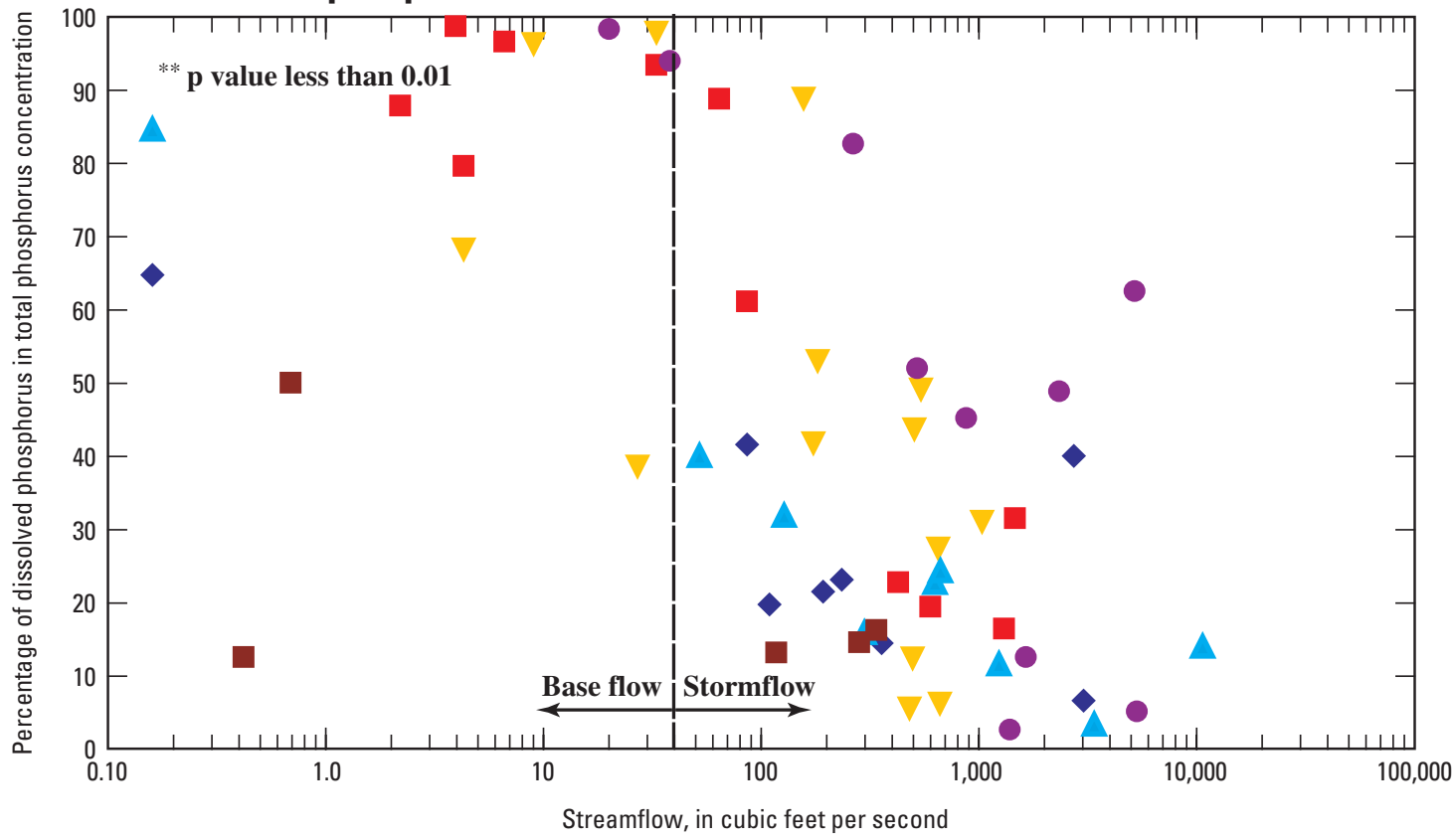

\section{EXPLANATION}

Stormflow sampling sites (fig. 1)

$$
\begin{aligned}
& \triangle \mathrm{BL} 5 \bullet \mathrm{KI} 6 \mathrm{~b} \\
& \text { CE6 } \nabla \text { MI7 } \\
& \text { - IN6 TU1* } \\
& \text { Note: * One value is omitted from site TU1 } \\
& \text { because sampling lacked corresponding } \\
& \text { streamflow measurement. }
\end{aligned}
$$

Figure 14. Percentage of dissolved nitrogen and dissolved phosphorus species in total nitrogen and phosphorus concentrations in samples collected at stormflow sampling sites under varying streamflow conditions, October 2002-June 2004. 
A. Big Bull Creek watershed

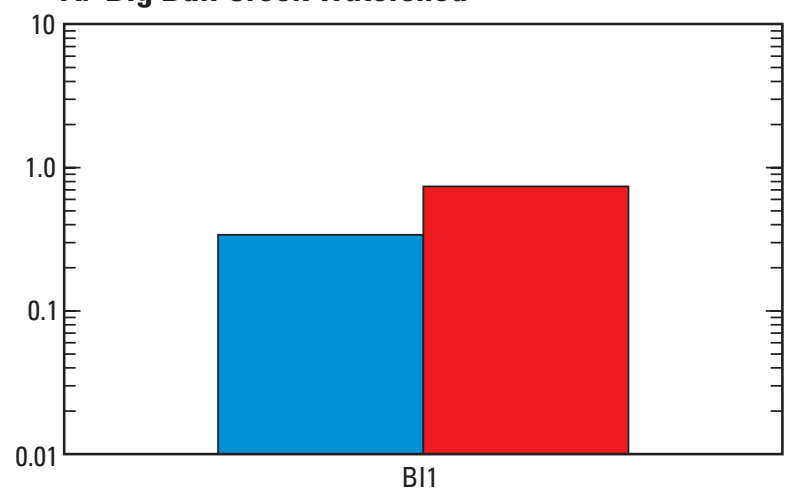

C. Brush Creek watershed

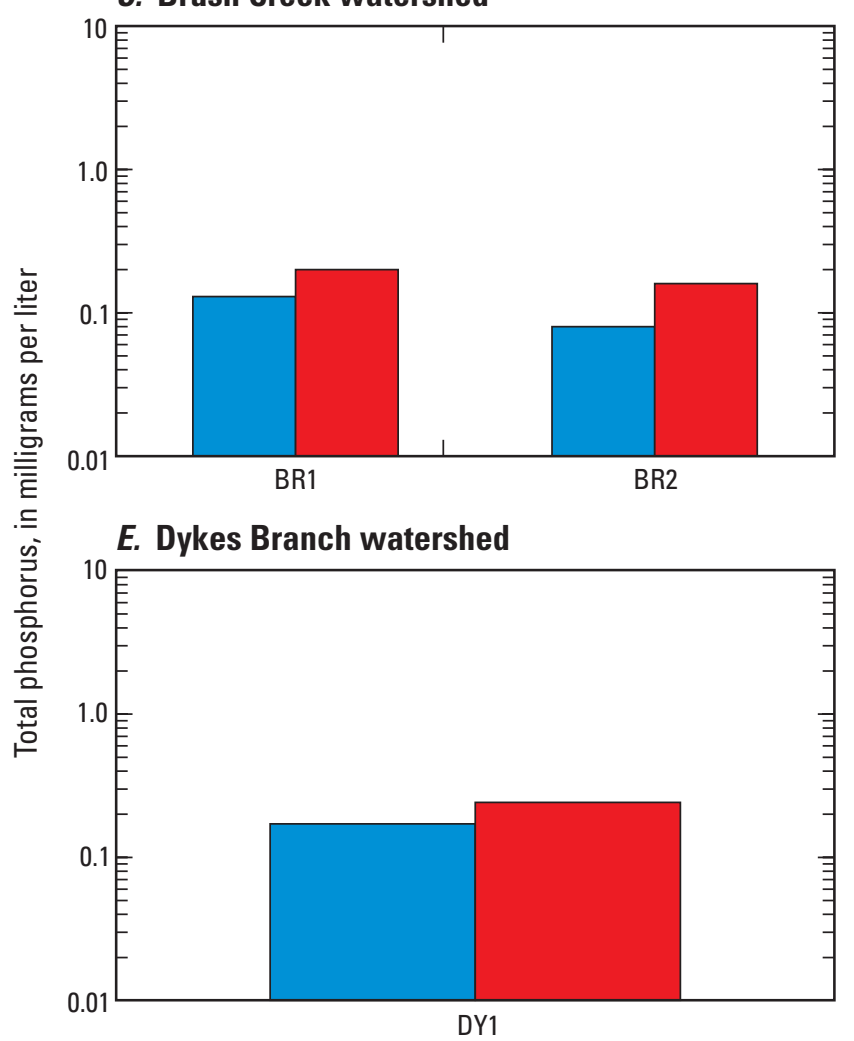

G. Kill Creek watershed

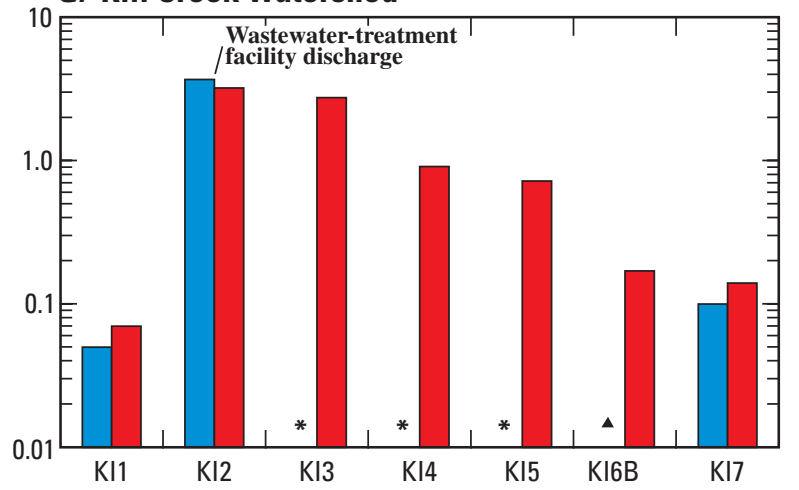

B. Blue River watershed

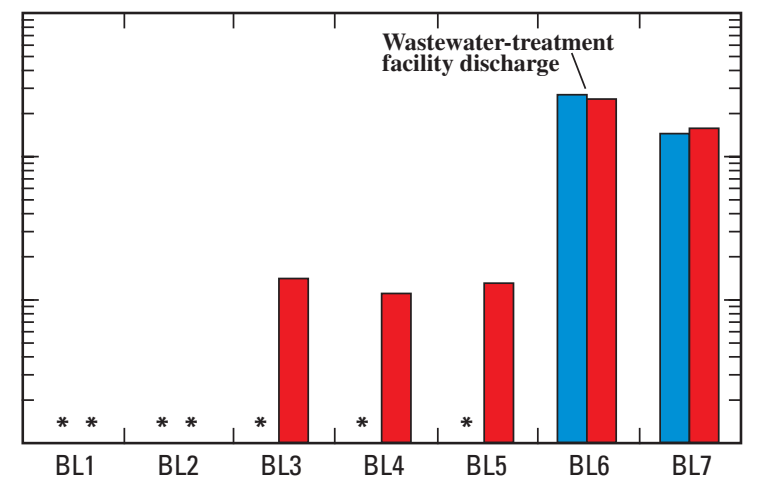

D. Cedar Creek watershed

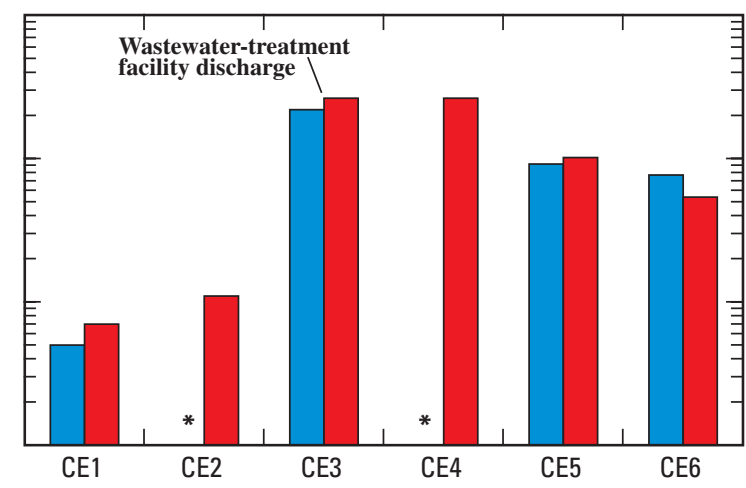

F. Indian Creek watershed

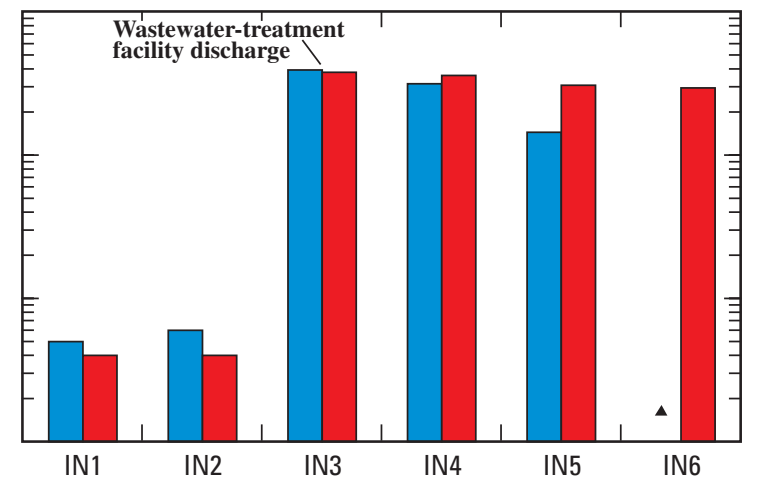

H. Little Bull Creek watershed

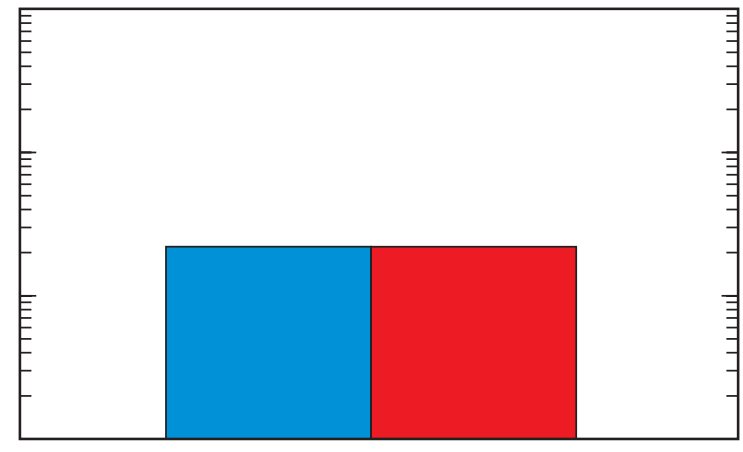

LI1

Sampling site (fig. 1)

Figure 15. Total phosphorus concentrations in base-flow samples, November 4-7, 2002, and July 14-18, 2003. 


\section{Mill Creek watershed}

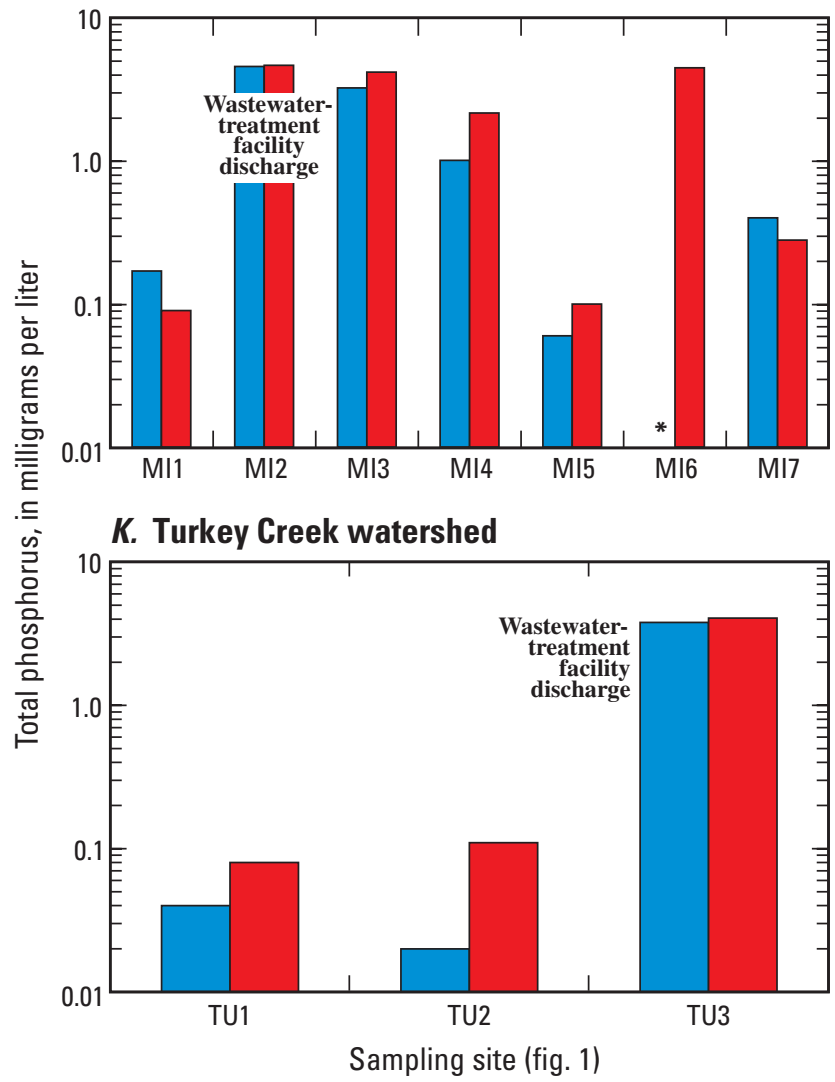

J. Tomahawk Creek watershed

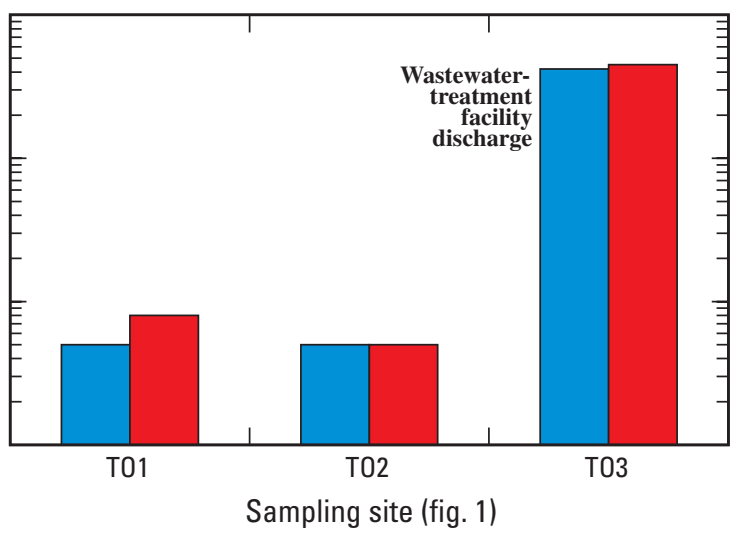

EXPLANATION

Synoptic survey

November 4-7, 2002

July 14-18, 2003

* No observable flow-sample not collected

- Site only sampled during second synoptic survey

Figure 15. Total phosphorus concentrations in base-flow samples, November 4-7, 2002, and July 14-18, 2003.-Continued

geometric mean of $200 \mathrm{col} / 100 \mathrm{~mL}$ in WWTF discharge. Earlier KDHE bacteria criteria for stream water mandated no more than a geometric mean of $200 \mathrm{col} / 100 \mathrm{~mL}$ for primary contact recreation, and the secondary contact criterion was a singlesample standard of 2,000 col/100 mL (Kansas Department of Health and Environment, 2002). Although these criteria are no longer used to regulate water quality in streams, they can be used as a guideline for acceptable fecal coliform densities and for comparison to new $E$. coli criteria.

Current (2005) Kansas stream-water-quality criteria for E. coli are divided into both primary and secondary contact criteria, and additionally into various classes $(\mathrm{B}, \mathrm{C})$ that indicate the use and accessibility of each stream. Primary contact recreation criteria refer to activities such as boating, mussel harvesting, swimming, skin diving, waterskiing, and windsurfing, whereas secondary contact criteria are applicable to activities such as wading, fishing, and hunting. Primary contact class B regulations are relevant for streams that are accessible under Kansas law, secondary contact class B and primary contact class $\mathrm{C}$ regulations are applicable to streams that are not accessible under Kansas law.

Criteria for primary contact class B streams in Kansas state that the geometric mean (the exponent of the mean of logarithmically transformed data) for $E$. coli bacteria of at least five samples collected over separate 24 -hour periods during a 30-day period shall not exceed $262 \mathrm{col} / 100 \mathrm{~mL}$ from April 1 through October 31 and 2,358 col/100 $\mathrm{mL}$ from November 1 through March 31 . The secondary contact class B criterion for E. coli may not exceed a geometric mean of $3,843 \mathrm{col} / 100 \mathrm{~mL}$ during any part of the year (Kansas Department of Health and Environment, 2004a).

Primary and secondary contact class B criteria for E. coli were used as a reference against samples from all Johnson County streams to simplify site-to-site comparisons, although many streams are subject to class $\mathrm{C}$ criteria (table 2). Bacteria samples collected during base-flow synoptic surveys do not represent a geometric mean of bacteria samples and are not subject to bacteria criteria. However, these criteria are used for the purpose of comparison to indicator bacteria densities determined in this study (Rasmussen and Ziegler, 2003). Along with E. coli, enterococci are the indicator bacteria recommended by USEPA and have been shown to correlate with enteric illnesses in freshwater lake studies (U.S. Environmental Protection Agency, 1986).

Sources of indicator bacteria in base-flow samples were not as traceable to WWTF discharge as were dissolved solids and nutrients. Class B primary contact criterion (for April 1 through October 31) and secondary contact criterion (any time of year) were used for comparison to base- and stormflow samples (figs. 18 and 19; table 15). Sites located upstream from WWTF discharges exceeded fecal coliform and $E$. coli criteria more frequently; Mann-Whitney statistical tests showed that 


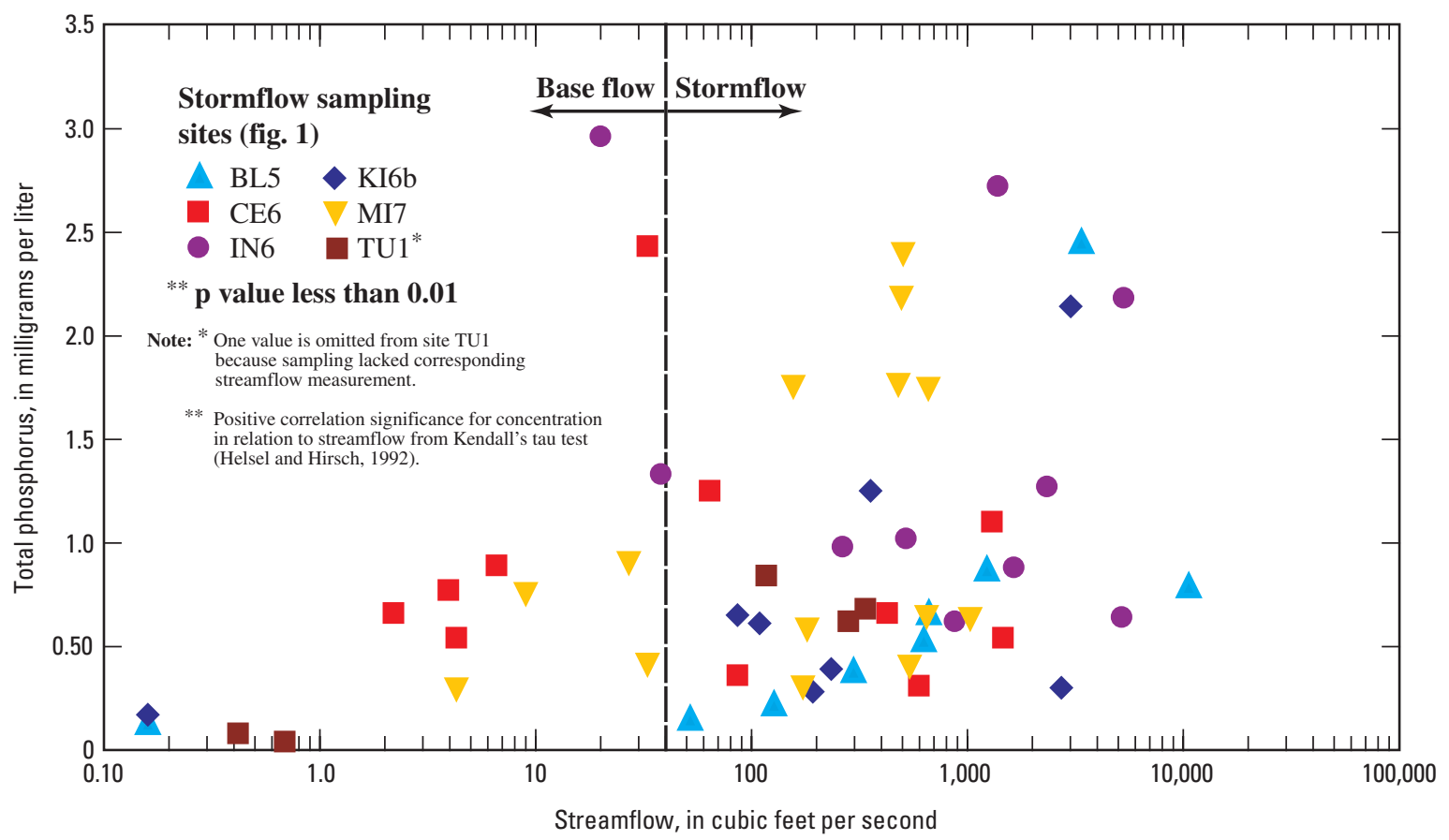

Figure 16. Total phosphorus concentrations in base-flow and stormflow samples collected at stormflow sampling sites, October 2002-June 2004.
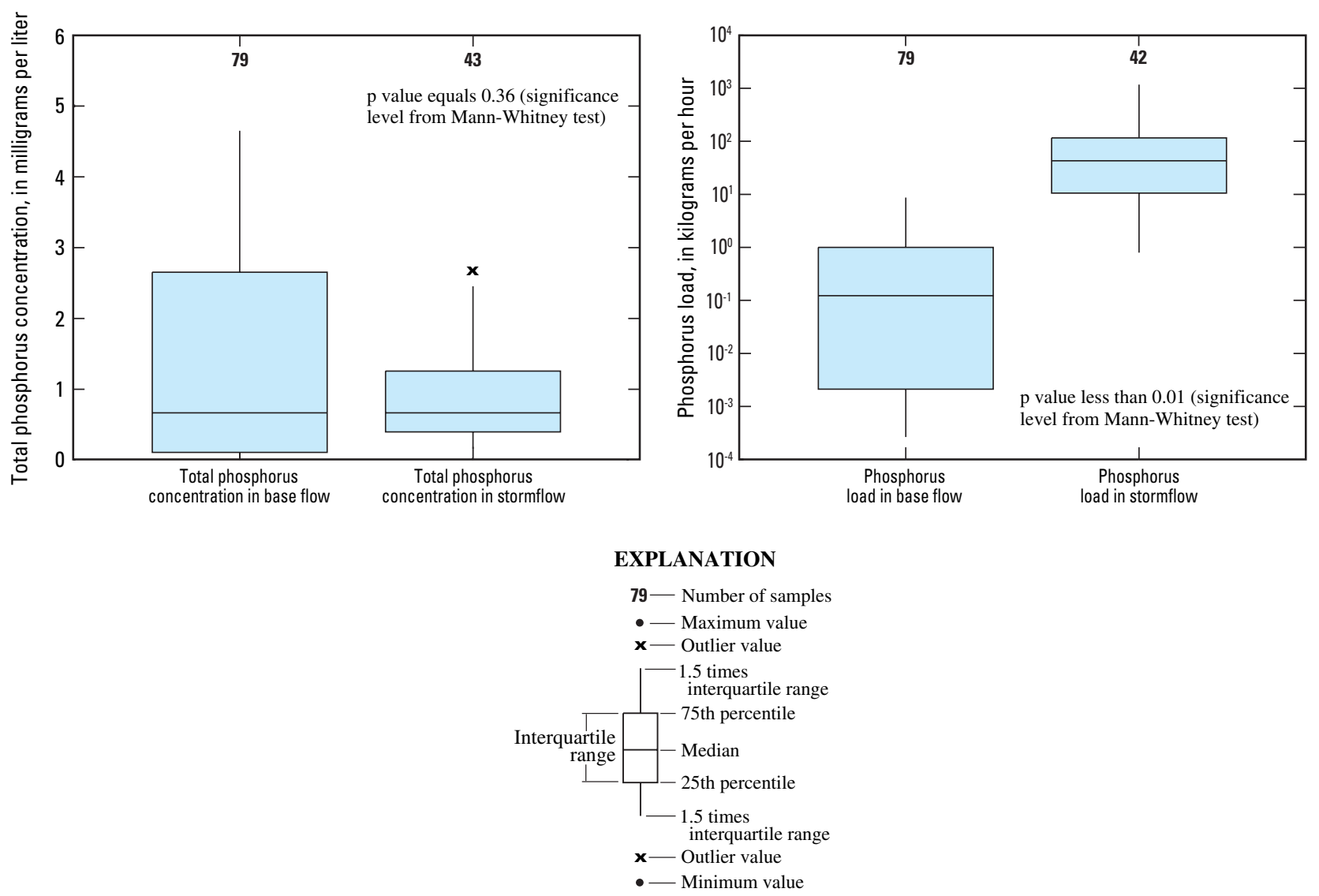

Figure 17. Total phosphorus concentrations and estimated loads in base- and stormflow samples, October 2002-June 2004. 


\section{A. Big Bull Creek watershed}

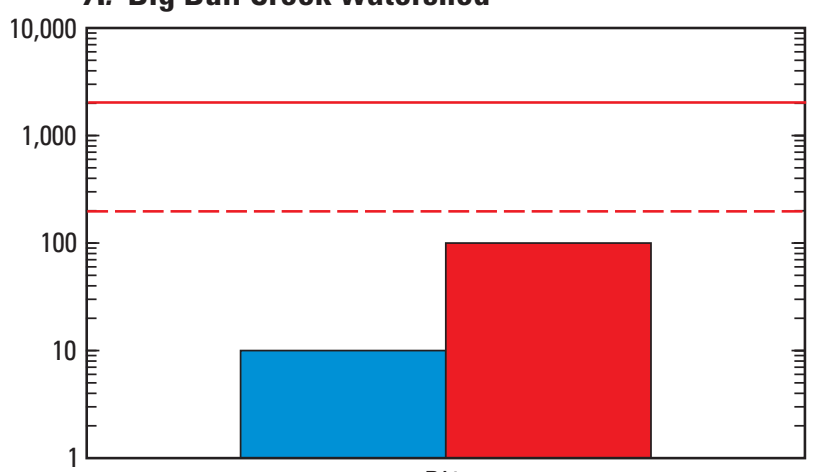

BI1

\section{Brush Creek watershed}

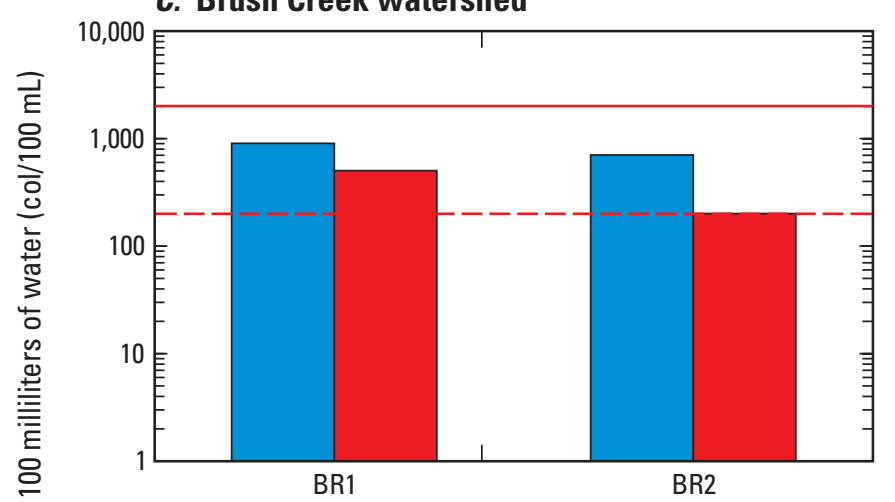

E. Dykes Branch watershed
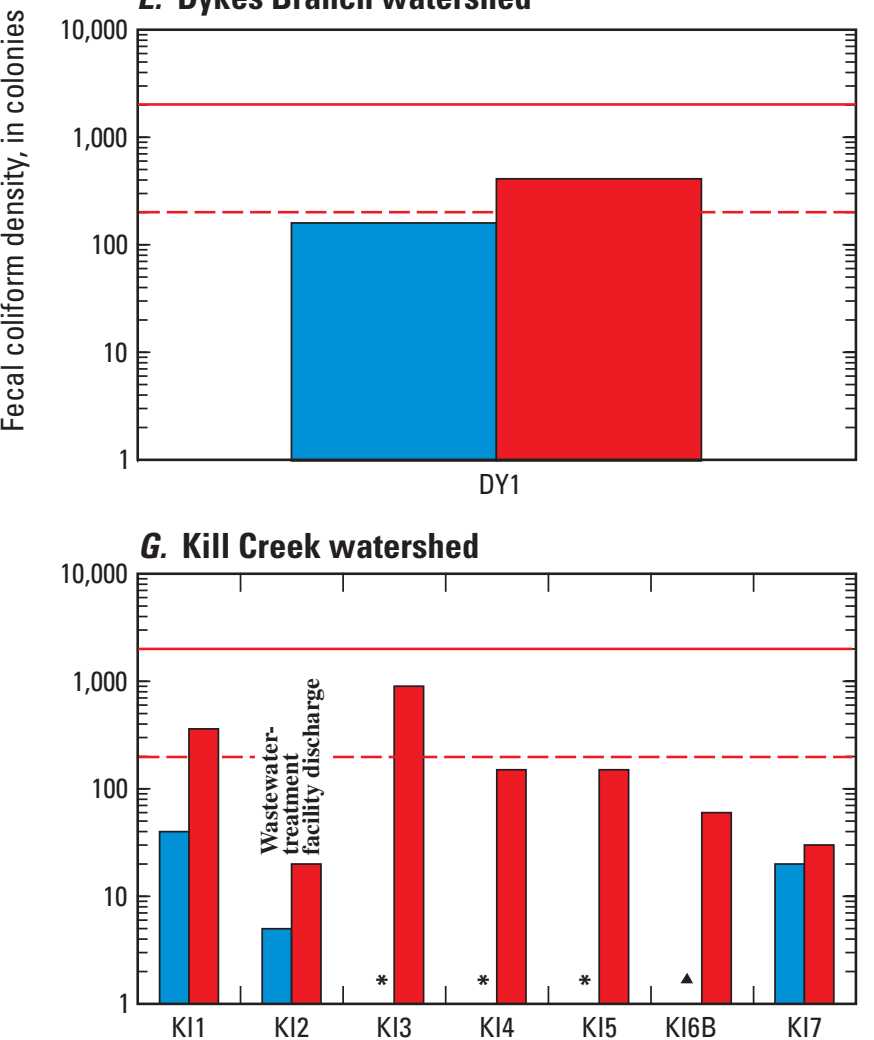

B. Blue River watershed

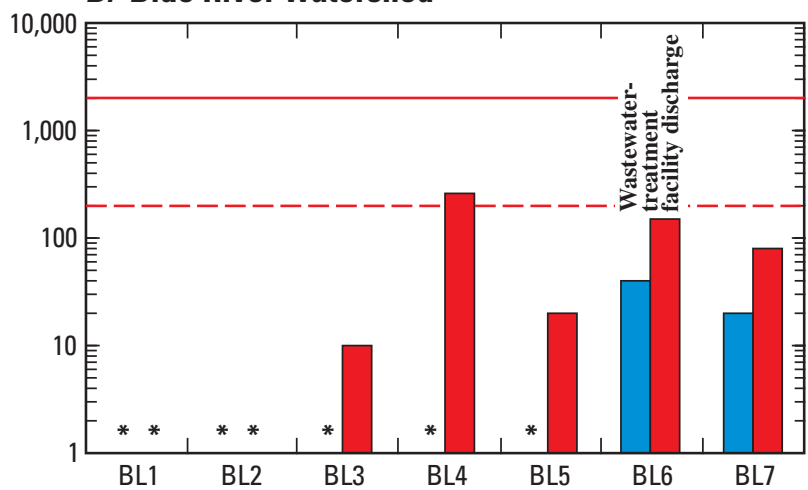

D. Cedar Creek watershed
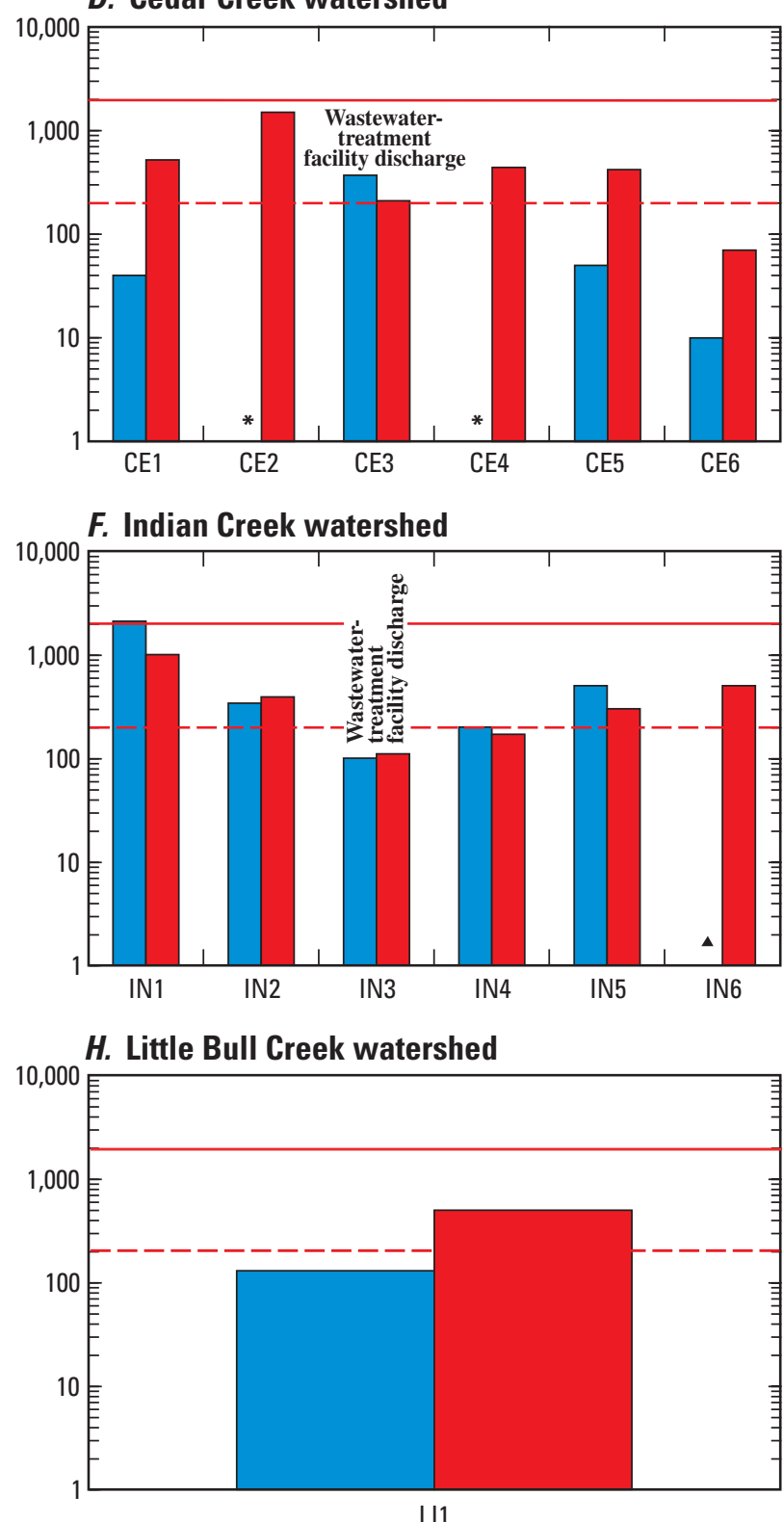

Sampling site (fig. 1)

Figure 18. Fecal coliform bacteria densities in base-flow samples, November 4-7, 2002, and July 14-18, 2003. 
I. Mill Creek watershed

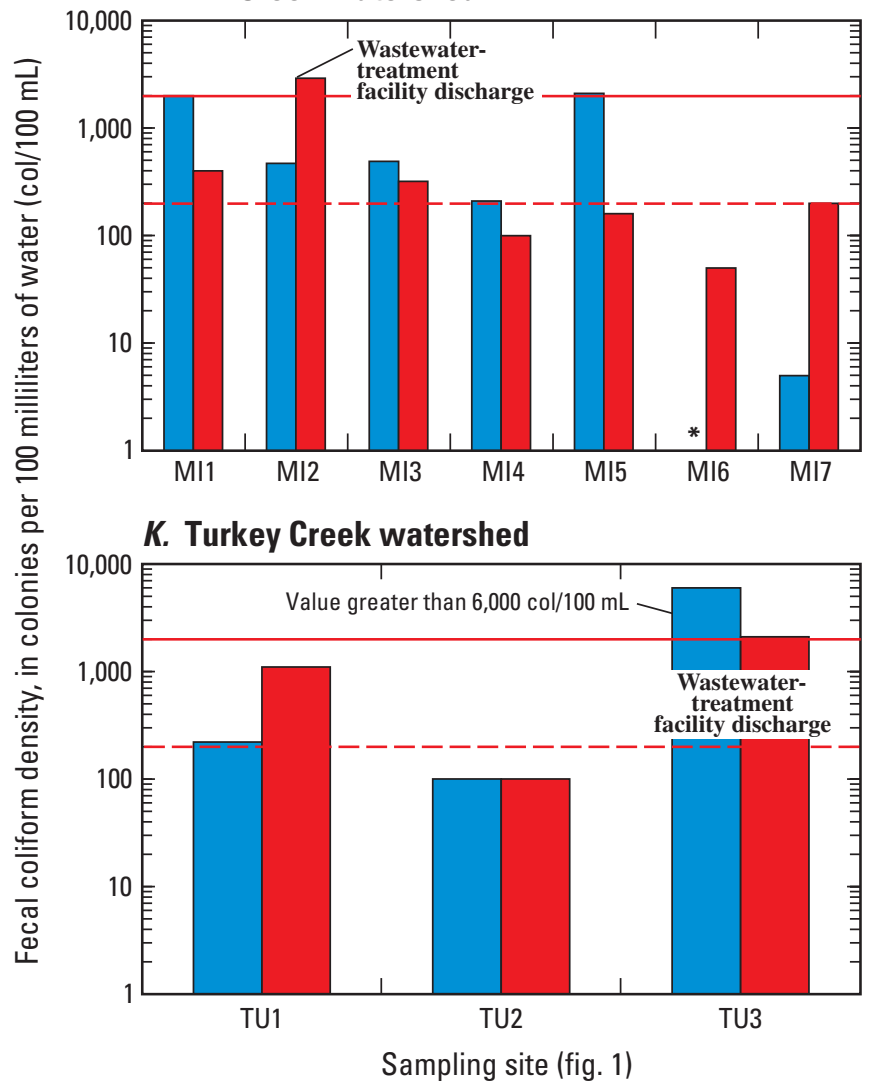

J. Tomahawk Creek watershed

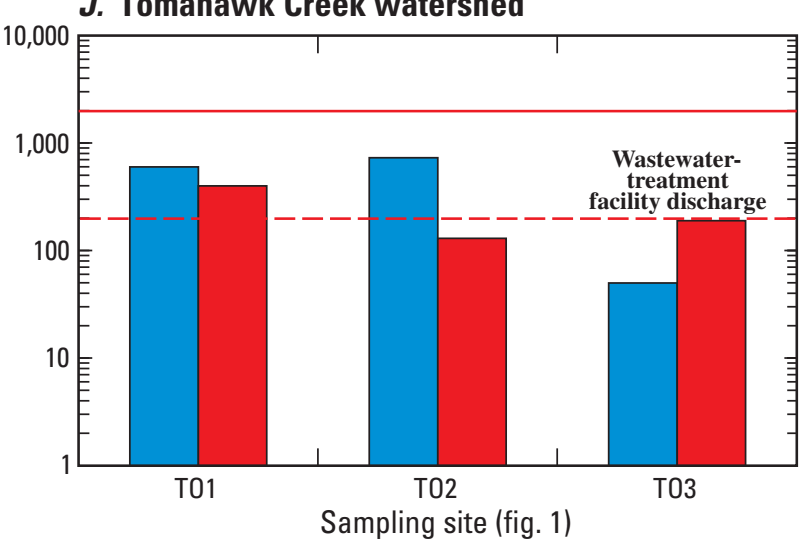

EXPLANATION

November 4-7, 2002

July 14-18, 2003

Fecal coliform secondary contact 2002 criterion $(2,000 \mathrm{col} / 100 \mathrm{~mL})$

--- Fecal coliform primary contact criterion $(200 \mathrm{col} / 100 \mathrm{~mL}$, applicable April 1 through October 31)

* No observable flow-sample not collected

- Site only sampled during second synoptic survey

Figure 18. Fecal coliform bacteria densities in base-flow samples, November 4-7, 2002, and July 14-18, 2003.—Continued

upstream sites had significantly larger median ( $\mathrm{p}$ value less than 0.05 ) bacteria densities than sites downstream from WWTF discharges (table 15). Of the seven WWTFs sampled, only samples from WWTF sites MI2 (420 and 1,100 col/100 mL) and TU3 $(16,300$ and $920 \mathrm{col} / 100 \mathrm{~mL})$ had $E$. coli densities that exceeded the class B primary or secondary contact recreation criteria (fig. 19). Among sites upstream from WWTF discharges, site MI5 (2,700 col/100 mL), site IN1 $(1,400 \mathrm{col} / 100 \mathrm{~mL})$, and site MI1 $(1,200 \mathrm{col} / 100 \mathrm{~mL})$ had the largest $E$. coli densities during November base-flow sampling. Indicator bacteria concentrations in samples from the other five WWTF discharge sites were among the smallest in their respective watersheds because of disinfection procedures.

Enterococci densities in Johnson County streams were compared to USEPA recommended criterion; 45 of 73 enterococci base-flow samples exceeded USEPA infrequently used full-body contact criterion of $151 \mathrm{col} / 100 \mathrm{~mL}$ (U.S. Environmental Protection Agency, 1986). The smallest enterococci densities generally were observed in samples from WWTF sites with the exception of densities in samples from site TU3.

Land-use differences were an important factor in the magnitude of base-flow fecal coliform and E. coli densities. Median indicator bacteria densities were compared at predominantly urban or predominantly nonurban sites where wastewater discharges had not increased ambient bacteria densities (table 16). Therefore, for the purpose of the analysis presented in table 16, all WWTF discharge sites and sites downstream from site MI2 (figs. 18 and 19) were removed. In addition, Blue River sites were classified as being upstream from WWTF discharge because the municipal WWTF discharge upstream from site BL2 (fig. 1) did not contribute measurable streamflow during base-flow conditions (fig. 5). Median fecal coliform and E. coli densities were significantly (p value less than 0.01 ) larger at predominantly urban sites than at sites with predominantly nonurban land uses, and enterococci densities were not significantly larger ( $\mathrm{p}$ value $=0.07$ ) in urban areas compared to nonurban areas (table 16). This comparison indicates that, although indicator bacteria densities are ubiquitous in the environment, potential effects from urban sources (such as leaking sewer lines, pet waste, surface-water and sediment interactions, or illicit dumping) may cause larger densities of fecal coliform and E. coli during base-flow conditions than effects from nonurban sources (such as septic systems, domestic livestock, and wildlife).

Thirty-four of 56 samples collected at stormflow sampling sites and 33 of 37 samples collected at flows larger than $100 \mathrm{ft}^{3} / \mathrm{s}$ contained fecal coliform densities larger than the 2002 KDHE criterion of 2,000 col/100 mL (fig. 20A). Twenty-five of 56 samples collected during all flow conditions and 25 of 37 samples collected at flows greater than $100 \mathrm{ft}^{3} / \mathrm{s}$ contained E. coli densities larger than the class B secondary contact criterion (3,843 col/100 mL) established by KDHE (fig. 20B). 


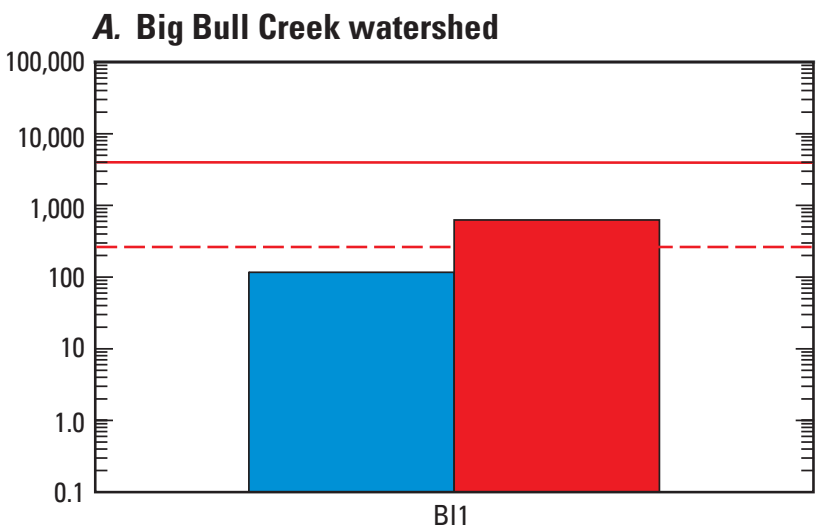

\section{B. Blue River watershed}

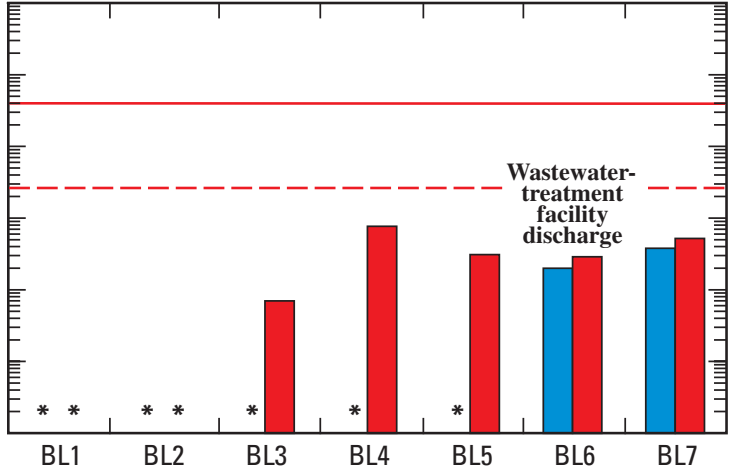

C. Brush Creek watershed

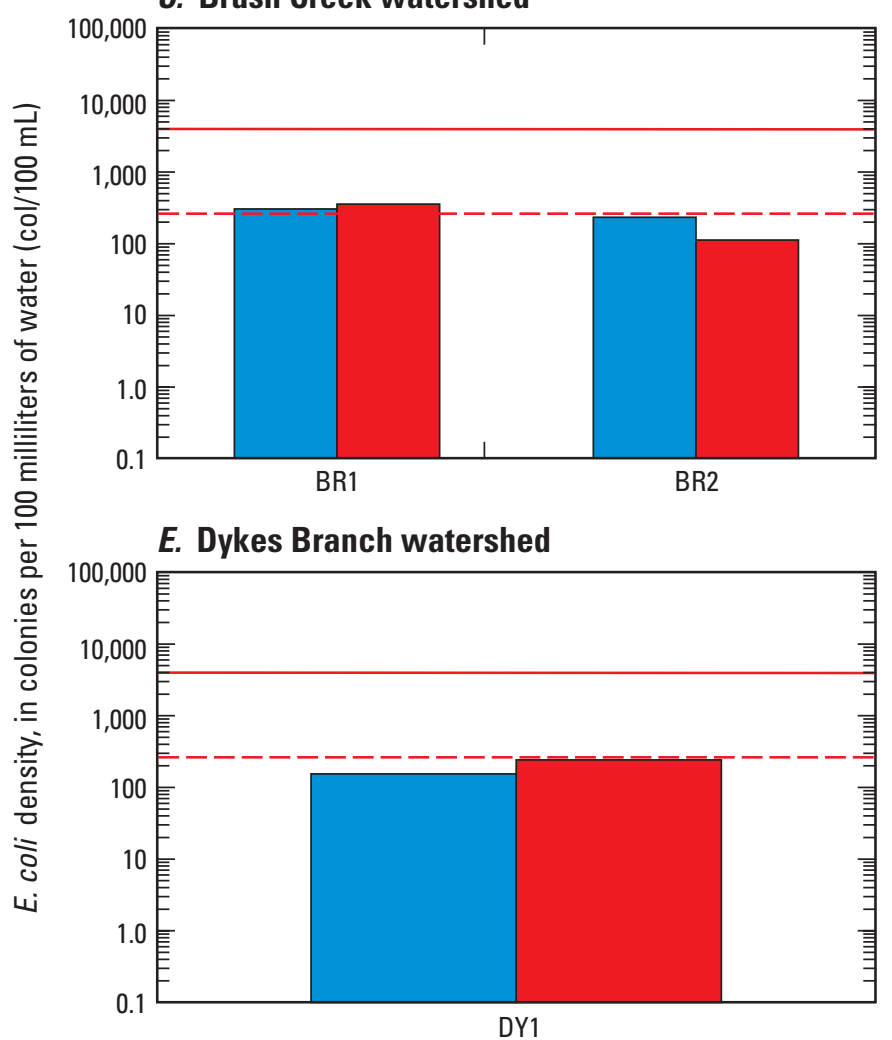

G. Kill Creek watershed

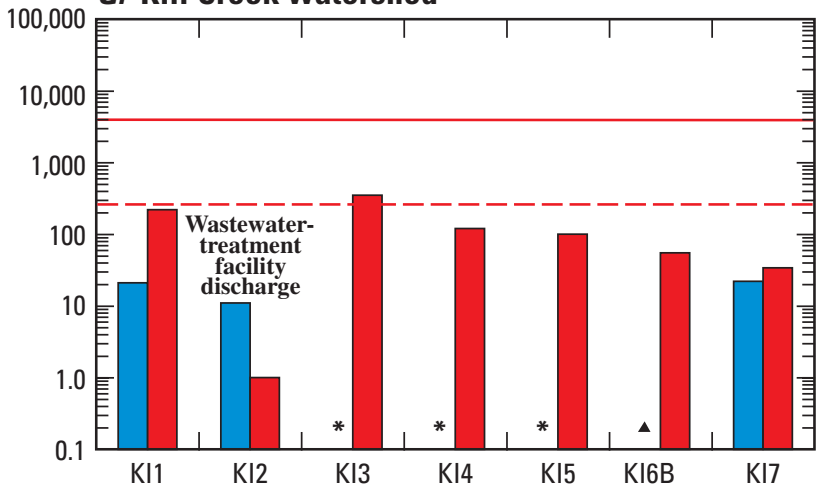

Sampling site (fig. 1)

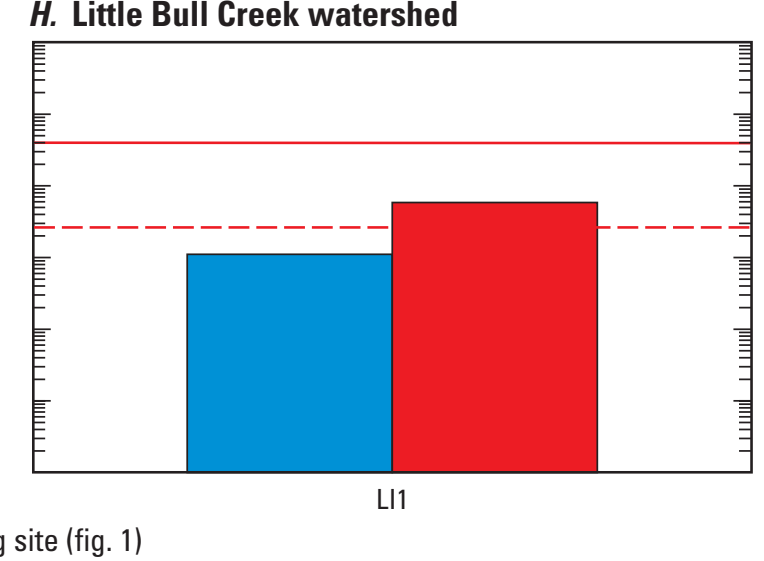

Figure 19. Escherichia coli (E. coli) densities in base-flow samples, November 4-7, 2002, and July 14-18, 2003. 

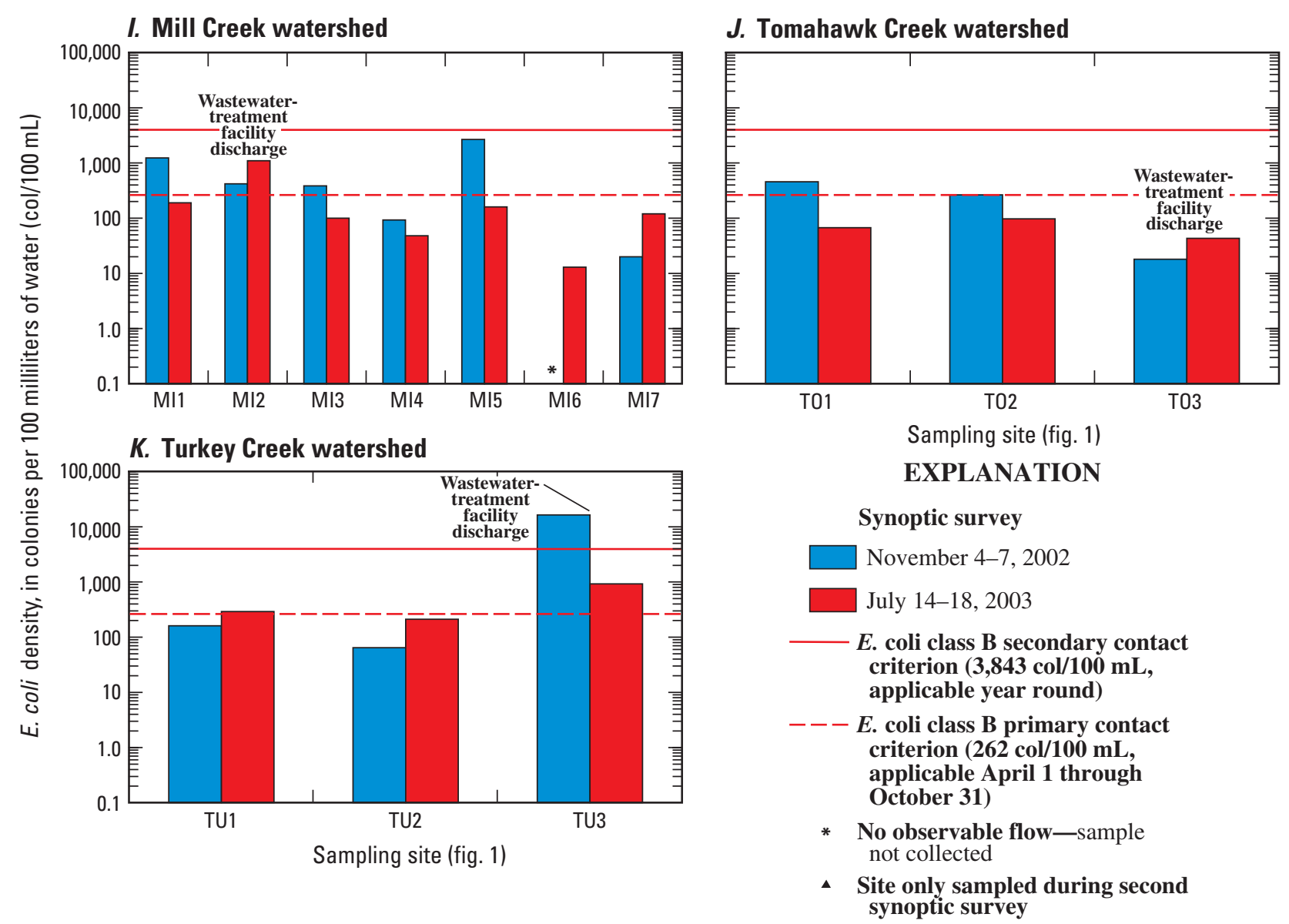

Figure 19. Escherichia coli (E. coli) densities in base-flow samples, November 4-7, 2002, and July 14-18, 2003.—Continued

Maximum bacteria densities were within an order of magnitude for each of the stormflow sampling sites. E. coli densities averaged 77 percent of fecal coliform densities. Enterococci densities were larger than USEPA (1986) infrequently used full-body contact recreation criterion of $151 \mathrm{col} / 100 \mathrm{~mL}$ in 46 of 56 samples collected at stormflow sampling sites ( 37 of 37 samples collected at flows larger than $100 \mathrm{ft}^{3} / \mathrm{s}$ ), whereas 36 of 56 samples had densities larger than 10,000 col/100 mL (fig. 20C). Enterococci densities were generally larger than other indicator bacteria in stormflow samples; densities in stormflow samples were an average of 14.4 times that of fecal coliform densities and 12.4 times that of $E$. coli densities. Samples from stormflow sampling sites showed that $E$. coli densities and loads were significantly larger (two to four orders of magnitude) in stormflow than base-flow samples ( $p$ value less than 0.01) (fig. 21), indicating that the largest contributions of $E$. coli were likely from nonpoint sources and (or) sanitary sewer overflows.

Although indicator bacteria densities were larger during stormflow conditions, there was increasing variability at large streamflow values, indicating that streamflow may not be the dominant variable affecting bacteria densities. Indicator bacteria were compared with suspended-sediment concentrations to contrast this relation with that of indicator bacteria and streamflow values. Previous studies indicated that bacteria populations in streams are closely correlated to suspended sediment (Christensen and others, 2000). Regression models and coefficients of determination $\left(\mathrm{R}^{2}\right)$ for models estimating indicator bacteria concentrations were determined using streamflow and suspended-sediment concentration (table 17). Suspendedsediment concentration had a better relation to indicator bacteria densities than streamflow, indicating that the occurrence of indicator bacteria densities in Johnson County streams is more closely related to suspended-sediment concentrations. This relation indicates that the occurrence of indicator bacteria may be tied to the occurrence of suspended sediment during stormflow conditions and that these constituents may have similar sources. Factors such as seasonality, water salinity, bacteria mortality characteristics, sanitary sewer overflows, leaking sewer lines, WWTF bypasses, and analytical method variability may affect the validity of this relation on both a temporal and spatial basis.

\section{Pesticides}

An estimated 2,103 million $\mathrm{lb}$ of pesticides were applied in the United States in 2001, the majority of which were herbicides (58 percent), insecticides ( 28 percent), and fungicides ( 8 percent) (Kiely and others, 2004). It is estimated that 76 percent of the United States total annual pesticide use in 2001 was for 
Table 15. Percentage of base-flow samples collected up- and downstream from wastewater-treatment discharges that were equal to or exceeded Kansas Department of Health and Environment (2002, 2003a) Escherichia coli (E. coli) and prior fecal coliform criteria for selected Johnson County streams, northeastern Kansas, October 2002-June 2004.

[col/100 mL, colonies per 100 milliliters of water; --, not applicable]

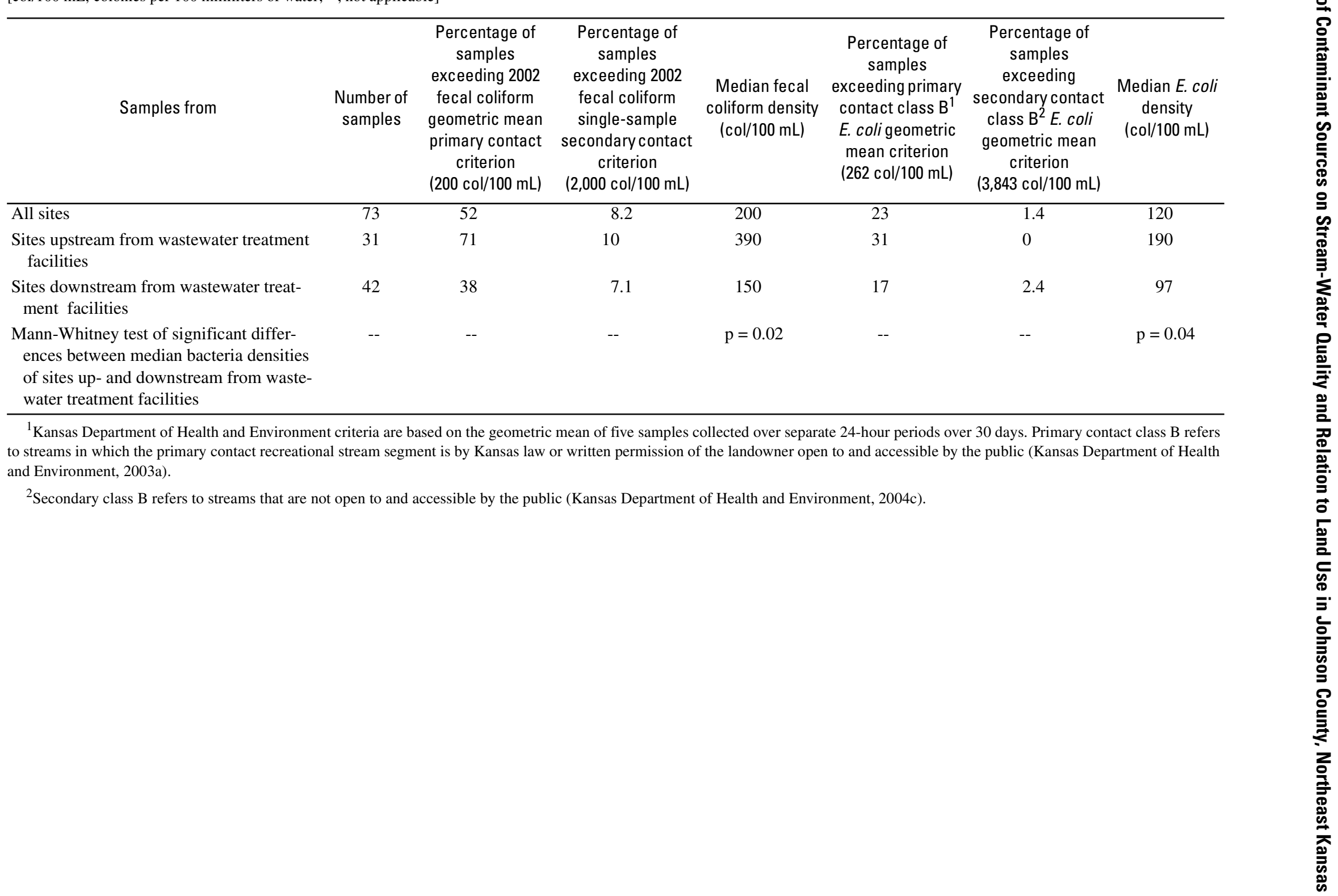


Table 16. Median indicator bacteria densities in base-flow samples from watersheds with predominantly urban or nonurban land uses, Johnson County, northeastern Kansas, November 4-7, 2002, and July 14-18, 2003.

[col/100 mL, colonies per 100 milliliters of water; --, not applicable; <, less than]

\begin{tabular}{|c|c|c|c|c|c|}
\hline $\begin{array}{l}\text { Predominant land use } \\
\text { in watershed } \\
\text { (fig. 2) }\end{array}$ & $\begin{array}{l}\text { Estimated mean } \\
\text { percentage of } \\
\text { urban land }{ }^{1} \\
\text { (residential, } \\
\text { commercial, } \\
\text { industrial, } \\
\text { right-of-way) }\end{array}$ & $\begin{array}{l}\text { Sampling site } \\
\text { identifier } \\
\text { (fig. 1) }\end{array}$ & $\begin{array}{l}\text { Median fecal } \\
\text { coliform density } \\
\text { (col/100 mL) }\end{array}$ & $\begin{array}{c}\text { Median } \\
\text { Escherichia coli } \\
\text { density } \\
\text { (col/100 mL) }\end{array}$ & $\begin{array}{c}\text { Median } \\
\text { enterococci } \\
\text { density } \\
\text { (col/100 mL) }\end{array}$ \\
\hline Urban & 82 & $\begin{array}{l}\text { BR1, BR2, DY1, } \\
\text { IN1, IN2, IN4, } \\
\text { IN5, IN6, MI, } \\
\text { TO1, TO2, TU1, } \\
\text { TU2 }\end{array}$ & 400 & 230 & 370 \\
\hline Nonurban & 28 & $\begin{array}{l}\text { BI1, BL3, BL4, } \\
\text { BL5, BL7, CE1, } \\
\text { CE2, CE4, CE5, } \\
\text { CE6, KI1, KI3, } \\
\text { KI4, KI5, KI6b, } \\
\text { KI7, LI1 }\end{array}$ & 80 & 52 & 190 \\
\hline $\begin{array}{l}\text { Mann-Whitney test of } \\
\text { significant differ- } \\
\text { ences between } \\
\text { median bacteria } \\
\text { densities of urban } \\
\text { and rural sites }\end{array}$ & -- & -- & $\mathrm{p}<0.01$ & $\mathrm{p}<0.01$ & $\mathrm{p}=0.07$ \\
\hline
\end{tabular}

agricultural purposes, 13 percent for commercial/industrial/ government use, and 11 percent for home or garden use (Kiely and others, 2004). Pesticide use is a concern in Johnson County due to the potential effects on aquatic organisms, bioaccumulation in fish tissue, and migration to downstream drinking-water supplies.

Median and maximum pesticide concentrations in baseflow and stormflow samples collected from Johnson County streams during October 2002-June 2004 were compared to data collected nationwide during the USGS NAWQA cycle-I studies from 1992-2001 (Martin and others, 2003) to place Johnson County samples in a national context (table 18). NAWQA information on frequently analyzed pesticides was grouped by type of sampling site (urban or agricultural) and represents a minimum of 135 samples.

Ninety-three pesticide compounds were analyzed in stream-water samples collected during base-flow synoptic surveys (table 6). Pesticide laboratory analyses were changed between the November 2002 and July 2003 synoptic surveys, so many compounds were analyzed exclusively during either the first or second survey. Pesticide compounds were analyzed during the first survey in base-flow samples collected from 31 sites and in samples from 22 sites during the second survey.

A majority of pesticide compounds either were not detected or were estimated at less than the laboratory reporting levels in base-flow samples. Pesticide detections were fewer and of a smaller magnitude in samples collected during the first synoptic survey in November 2002, before spring and summer pesticide application. Because of the large number of pesticides analyzed, only selected compounds are discussed in this section.

The pesticide atrazine proved to be an accurate representative of pesticide sources and transport in Johnson County streams. Atrazine was the most frequently detected pesticide in surface water in the early 1990s, has little tendency to adsorb to aquatic particles or volatilize to air, and is not prone to chemical or microbiological transformation processes (Capel and Larson, 2001).

Atrazine was detected in all Johnson County base-flow samples (fig. 22) and had the largest concentrations of any pesticide sampled (table 18). However, only one atrazine baseflow sample collected during the July synoptic at site BI1 $(3.5 \mu \mathrm{g} / \mathrm{L})$ exceeded the KDHE chronic aquatic-life criterion of $3.0 \mu \mathrm{g} / \mathrm{L}$ (Kansas Department of Health and Environment, 2004c). The atrazine concentration in this sample was more than four times that in any other base-flow sample. 2-Chloro-4isopropylamino-6-amino-s-triazine (atrazine metabolite) and acetochlor were detected at relatively large concentrations in this sample. These concentrations may be due to agricultural pesticide uses upstream from of this site; Putnam (1997) found triazine herbicide concentrations greater than $3.0 \mu \mathrm{g} / \mathrm{L}$ in Big Bull Creek in May 1994 and 1995. 

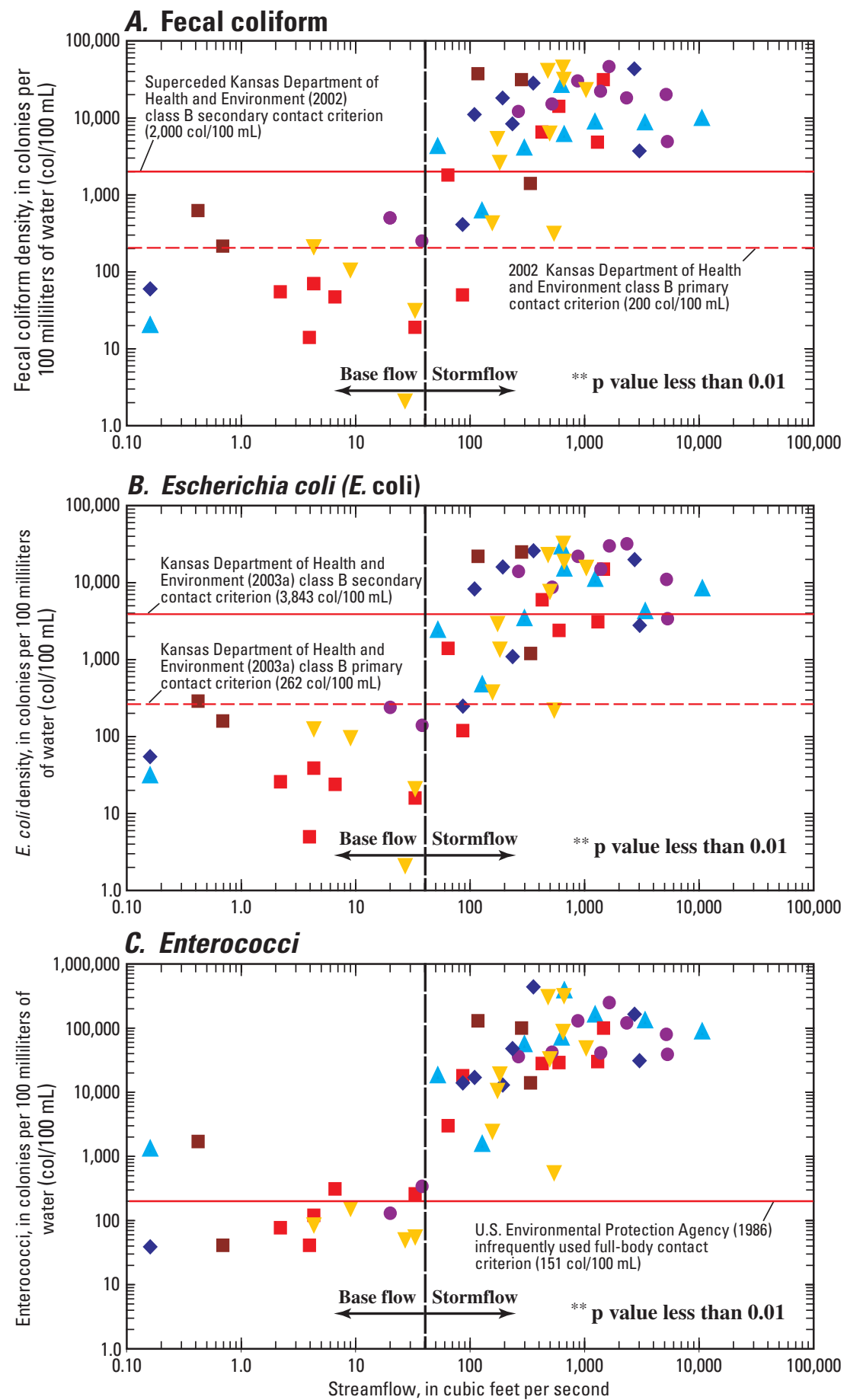

EXPLANATION

Stormflow sampling sites (fig. 1)

$$
\begin{array}{ll}
\text { BL5 } & \text { KI6b } \\
\text { CE6 } & \nabla \text { MI7 } \\
\text { - IN6 } & \text { TU1* }
\end{array}
$$

Note: ${ }^{*}$ One value is omitted from site TU1 because sampling lacked corresponding streamflow measurement.

** Significance from linear regression analysis for density in relation to streamflow

Figure 20. Indicator bacteria densities in base-flow and stormflow samples collected at stormflow sampling sites, October 2002-June 2004. 

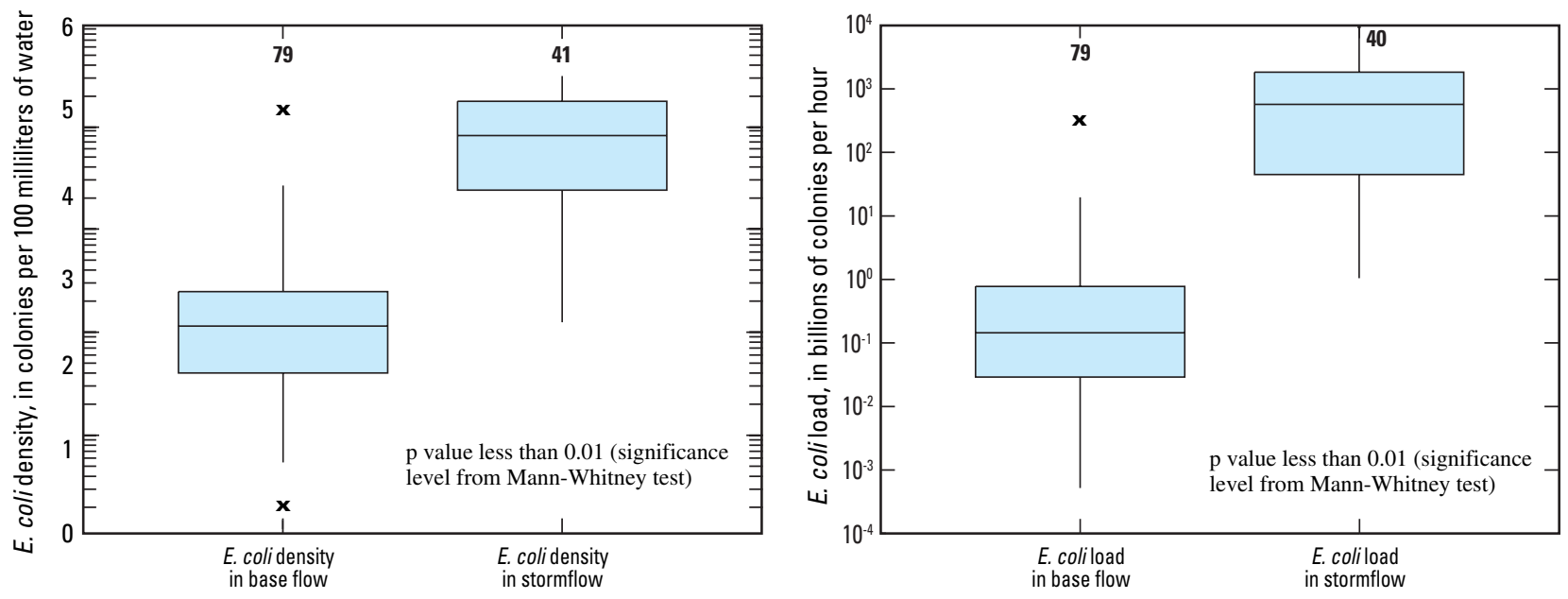

EXPLANATION

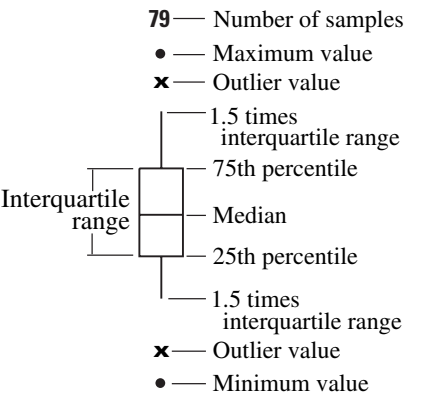

Figure 21. Escherichia coli (E. coli)densities and estimated loads in base- and stormflow samples, October 2002-June 2004.

Maximum atrazine concentrations in the Indian $(0.43 \mu \mathrm{g} / \mathrm{L})$, Kill $(0.55 \mu \mathrm{g} / \mathrm{L})$, Mill $(0.50 \mu \mathrm{g} / \mathrm{L})$, Tomahawk $(0.45 \mu \mathrm{g} / \mathrm{L})$, and Turkey Creek $(0.17 \mu \mathrm{g} / \mathrm{L})$ watersheds were in base-flow samples collected at WWTF discharge sites in July 2003 (fig. 22). Atrazine in wastewater discharge may have been due to concentrations in municipal water supplies;

concentrations ranged from 0.2 to $0.6 \mu \mathrm{g} / \mathrm{L}$ in water supplied by Water District No. 1 of Johnson County during 2004 (WaterOne, 2004). Blue River atrazine concentrations varied little in base-flow samples collected during the July 2003 synoptic survey upstream (site BL5, $0.15 \mu \mathrm{g} / \mathrm{L}$ ), at (site BL6, $0.15 \mu \mathrm{g} / \mathrm{L}$ ), or downstream (site BL7, $0.15 \mu \mathrm{g} / \mathrm{L}$ ) from the WWTF discharge, potentially due to extended aeration treatment processes. Cedar Creek had the largest atrazine concentrations during the July 2003 synoptic survey upstream from WWTF discharges in a sample from site CE1 $(0.72 \mu \mathrm{g} / \mathrm{L})$ and in a sample from the WWTF discharge site CE3 $(0.52 \mu \mathrm{g} / \mathrm{L})$.

Other pesticides exhibited occurrence patterns similar to atrazine. Concentrations of the pesticides 3, 4 dichloroaniline (only sampled during the first synoptic survey), diazinon, and metolachlor had the largest median and maximum concentrations in base-flow samples from WWTF discharge sites (table 18). Median and maximum simazine concentrations were larger in base-flow samples from sites upstream from WWTF discharges, but the majority of detections were less than laboratory reporting levels. Base-flow samples generally had similar median, but smaller maximum, pesticide concentrations than in either agricultural or urban samples from the NAWQA program (table 18).

Sixty-six pesticide compounds were analyzed in Johnson County stormflow samples (table 6). Thirty-three of the compounds were detected in stormflow samples; 27 compounds were detected at concentrations greater than laboratory reporting levels (table 18). Although the sheer number of pesticide detections do not give an indication of the total amount of pesticides in each sample, these data are useful to distinguish which samples contained a broad range of contaminants. Samples from urban sites IN6 (18 detections), MI7 (14 detections), and TU1 (14 detections) averaged the most pesticide detections of samples from stormflow sites, whereas samples from site KI6b averaged the fewest number of detections (9) (fig. 23A). However samples from agricultural sites had the largest total concentrations of pesticides; 9 of the 10 largest total concentrations of pesticides in stormflow samples occurred at sites BL5, CE6, and KI6b. The largest total concentration of pesticides occurred at site KI6b (71 $\mu \mathrm{g} / \mathrm{L}$ on May 25, 2004) (fig. 23B).

Most pesticide transport occurs over a relatively short time period, generally after the first few rains or irrigation following application (Schottler and others, 1994). The largest number of pesticides detected in stormflow were in samples collected in the spring. Two of 54 stormflow samples exceeded the $3.0-\mu \mathrm{g} / \mathrm{L}$ KDHE chronic aquatic-life criterion for atrazine $(3.9 \mu \mathrm{g} / \mathrm{L}$ in a sample from site BL5 on April 25, 2003, and $69 \mu \mathrm{g} / \mathrm{L}$ in a sample from site KI6b on May 25, 2004). Only five other 
northeastern Kansas, October 2002-June 2004.

$\left[\mathrm{R}^{2}\right.$, coefficient of determination; MSE, mean square error; RMAE, relative mean absolute error; $\mathrm{mg} / \mathrm{L}$, milligrams per liter; col/100 mL, colonies per $100 \mathrm{milliliter}$ of water]

\begin{tabular}{llcccc}
\hline \multicolumn{1}{c}{ Dependent variable } & Independent variable & $\mathrm{R}^{2}$ & $\begin{array}{c}\text { Number of } \\
\text { samples }\end{array}$ & Range of dependent variable & MSE \\
(percent)
\end{tabular}


Table 18. Median and maximum concentrations of pesticide compounds detected at greater than laboratory reporting levels in base-flow and stormflow samples collected from selected Johnson County streams, northeastern Kansas, October 2002-June 2004, and samples collected nationwide for the U.S. Geological Survey's National Water-Quality Assessment cycle-I studies, 1992-2001.

[ $\mu \mathrm{g} / \mathrm{L}$, micrograms per liter; <, less than; E, estimated; --, category already reported in table]

\begin{tabular}{|c|c|c|c|c|c|c|c|c|}
\hline \multirow{3}{*}{ Compound } & \multicolumn{4}{|c|}{ Johnson County study } & \multicolumn{4}{|c|}{$\begin{array}{c}\text { National Water-Quality Assessment cycle-I } \\
\text { studies }{ }^{1}\end{array}$} \\
\hline & \multirow[b]{2}{*}{$\begin{array}{l}\text { Number of } \\
\text { samples }\end{array}$} & \multirow{2}{*}{$\begin{array}{l}\text { Detection } \\
\text { frequency } \\
\text { (percent) }\end{array}$} & \multicolumn{2}{|c|}{ Concentration $(\mu \mathrm{g} / \mathrm{L})$} & \multicolumn{4}{|c|}{ Concentration $(\mu \mathrm{g} / \mathrm{L})$} \\
\hline & & & Median & $\begin{array}{c}\text { Maximum } \\
\text { (sampling site } \\
\text { identifier, fig. 1) }\end{array}$ & $\begin{array}{l}\text { Agricul- } \\
\text { tural } \\
\text { median }\end{array}$ & $\begin{array}{l}\text { Agricul- } \\
\text { tural } \\
\text { maximum }\end{array}$ & $\begin{array}{l}\text { Urban } \\
\text { median }\end{array}$ & $\begin{array}{l}\text { Urban } \\
\text { maximum }\end{array}$ \\
\hline \multicolumn{5}{|c|}{ Base-flow samples } & \multicolumn{4}{|c|}{ Various streamflow conditions } \\
\hline $\begin{array}{l}\text { 2-Chloro-4-isopropyl- } \\
\text { amino-6-amino-s- } \\
\text { triazine (CIAT) }\end{array}$ & 23 & 96 & 0.06 & $0.22(\mathrm{BI} 1)$ & -- & -- & -- & -- \\
\hline 3,4 Dichloroaniline & 22 & 100 & .09 & $.69(\mathrm{CE} 3)$ & -- & -- & -- & -- \\
\hline 4-Chloro-2-methylphenol & 22 & 45 & $<.006$ & $.01(\mathrm{TU} 1)$ & -- & -- & -- & -- \\
\hline Acetochlor & 22 & 18 & $<.006$ & $.57(\mathrm{BI} 1)$ & $<0.005$ & 25 & $<0.004$ & 0.31 \\
\hline Atrazine & 54 & 100 & .14 & $3.5(\mathrm{BI} 1)$ & .071 & E200 & .01 & 3.4 \\
\hline Desulfinylfipronil & 53 & 57 & .01 & $.02(\mathrm{KI} 2)$ & -- & -- & -- & -- \\
\hline Desulfinylfipronil amide & 22 & 27 & $<.009$ & $.02(\mathrm{KI} 2)$ & -- & -- & -- & -- \\
\hline Diazinon & 73 & 60 & .02 & E.30(CE5) & $<.005$ & 2.5 & .01 & 1.4 \\
\hline Fipronil sulfide & 53 & 58 & .01 & $.01(\mathrm{KI} 2)$ & -- & -- & -- & -- \\
\hline Fipronil sulfone & 53 & 57 & .01 & .03 (IN6) & -- & -- & -- & -- \\
\hline Fipronil & 53 & 77 & .03 & $.11(\mathrm{TO} 3)$ & -- & -- & -- & -- \\
\hline Malathion & 53 & 8 & $<.027$ & E.03 (IN1) & $<.027$ & .5 & $<.027$ & .63 \\
\hline Metolachlor & 73 & 78 & .04 & .20 (CE3) & .03 & E78 & .003 & 2.4 \\
\hline Myclobutanil & 22 & 27 & $<.008$ & .04 (BR2) & -- & -- & -- & -- \\
\hline Simazine & 53 & 40 & $<.005$ & .04 (IN1) & .01 & 5.8 & .01 & 9 \\
\hline Tebuthiuron & 53 & 32 & $<.02$ & .12 (MI4) & $<.016$ & E.95 & $<.016$ & E2.8 \\
\hline Terbuthylazine & 22 & 14 & $<.01$ & .62 (LI1) & -- & -- & -- & -- \\
\hline \multicolumn{5}{|c|}{ Stormflow samples } & \multicolumn{4}{|c|}{ Various streamflow conditions } \\
\hline $\begin{array}{l}\text { 2-Chloro-4-isopropyl- } \\
\text { amino-6-amino-s- } \\
\text { triazine (CIAT) }\end{array}$ & 46 & 85 & .03 & $.28(\mathrm{KI} 6 \mathrm{~b})$ & -- & -- & -- & -- \\
\hline $\begin{array}{l}\text { 2-Chloro-2,6- } \\
\text { diethylacetanilide }\end{array}$ & 46 & 2 & $<.005$ & $.02(\mathrm{KI} 6 \mathrm{~b})$ & -- & -- & -- & -- \\
\hline 3,4 Dichloroaniline & 46 & 59 & .01 & .51 (MI7) & -- & -- & -- & -- \\
\hline 4-Chloro-2-methylphenol & 46 & 35 & $<.006$ & E.02 (MI7) & -- & -- & -- & -- \\
\hline Acetochlor & 46 & 52 & .01 & 1.1 (KI6b) & $<.005$ & 25 & $<.004$ & .31 \\
\hline Alachlor & 46 & 52 & $<.005$ & .73 (BL5) & .001 & 11 & $<.005$ & .4 \\
\hline
\end{tabular}


Table 18. Median and maximum concentrations of pesticide compounds detected at greater than laboratory reporting levels in base-flow and stormflow samples collected from selected Johnson County streams, northeastern Kansas, October 2002-June 2004, and samples collected nationwide for the U.S. Geological Survey's National Water-Quality Assessment cycle-I studies, 1992-2001. -Continued

[ $\mu \mathrm{g} / \mathrm{L}$, micrograms per liter; <, less than; E, estimated; --, category not reported]

\begin{tabular}{|c|c|c|c|c|c|c|c|c|}
\hline \multirow{3}{*}{ Compound } & \multicolumn{4}{|c|}{ Johnson County study } & \multirow{2}{*}{\multicolumn{4}{|c|}{$\begin{array}{l}\text { National Water-Quality Assessment cycle- } \\
\text { I studies }{ }^{1} \\
\text { Concentration }(\mu \mathrm{g} / \mathrm{L})\end{array}$}} \\
\hline & \multirow[b]{2}{*}{$\begin{array}{c}\text { Number of } \\
\text { samples }\end{array}$} & \multirow[b]{2}{*}{$\begin{array}{l}\text { Detection } \\
\text { frequency } \\
\text { (percent) }\end{array}$} & \multicolumn{2}{|c|}{ Concentration $(\mu \mathrm{g} / \mathrm{L})$} & & & & \\
\hline & & & Median & $\begin{array}{l}\text { Maximum } \\
\text { (sampling site } \\
\text { identifier, } \\
\text { fig. 1) }\end{array}$ & $\begin{array}{l}\text { Agricul- } \\
\text { tural } \\
\text { median }\end{array}$ & $\begin{array}{l}\text { Agricul- } \\
\text { tural } \\
\text { maximum }\end{array}$ & $\begin{array}{l}\text { Urban } \\
\text { median }\end{array}$ & $\begin{array}{c}\text { Urban } \\
\text { maximum }\end{array}$ \\
\hline \multicolumn{5}{|c|}{ Stormflow samples } & \multicolumn{4}{|c|}{ Various streamflow conditions } \\
\hline Atrazine & 46 & 95 & 0.16 & 69 (KI6b) & 0.071 & E200 & 0.01 & 3.4 \\
\hline Benfluralin & 46 & 37 & $<.01$ & .01 (IN6) & $<.01$ & .008 & $<.01$ & .022 \\
\hline Carbaryl & 45 & 61 & $<.041$ & .29 (IN6) & $<.041$ & E5.2 & E.004 & E5.2 \\
\hline Chlorpyrifos & 46 & 9 & $<.005$ & $.02($ KI6b) & $<.005$ & .26 & $<.005$ & .3 \\
\hline DCPA & 46 & 35 & $<.003$ & $.01(\mathrm{KI} 6 \mathrm{~b}, \mathrm{MI} 7)$ & -- & -- & -- & -- \\
\hline Desulfinyl fipronil & 46 & 59 & .005 & .01 (CE6) & -- & -- & -- & -- \\
\hline Diazinon & 46 & 65 & .01 & $.31(\mathrm{CE} 6)$ & $<.005$ & 2.5 & .01 & 1.4 \\
\hline Dichlorvos & 44 & 27 & $<.011$ & E.12 (IN6) & -- & -- & -- & -- \\
\hline Fipronil & 46 & 76 & .01 & .06 (IN6 & -- & -- & -- & -- \\
\hline Fonofos & 46 & 2 & $<.003$ & .02 (TU1) & $<.003$ & $<.003$ & $<.003$ & .084 \\
\hline Malathion & 46 & 20 & $<.027$ & .03 (TU1) & $<.027$ & .5 & $<.027$ & .63 \\
\hline Metalaxyl & 46 & 9 & $<.05$ & E.05 (CE6) & -- & -- & -- & -- \\
\hline Metolachlor & 46 & 98 & .03 & 1.7 (BL5) & .03 & E78 & 0 & 2.4 \\
\hline Myclobutanil & 46 & 33 & $<.008$ & .04 (IN6) & -- & -- & -- & -- \\
\hline Pendimethalin & 46 & 39 & $<.022$ & .09 (TU1) & $<.020$ & 2 & $<.020$ & .37 \\
\hline Prometon & 46 & 93 & .02 & .16 (MI7) & .01 & .25 & .2 & E25 \\
\hline Prometryn & 46 & 4 & $<.005$ & .01 (BL5, MI7) & -- & -- & -- & -- \\
\hline Simazine & 45 & 80 & .01 & 4.6 (IN6) & .01 & 5.8 & .01 & 9 \\
\hline Tebuthiuron & 46 & 20 & $<.016$ & .07 (MI7) & $<.016$ & E.95 & $<.016$ & E2.8 \\
\hline Terbuthylazine & 45 & 7 & $<.01$ & .04 (MI7) & -- & -- & -- & -- \\
\hline Trifluralin & 46 & 54 & $<.09$ & .02 (KI6b) & $<.09$ & .17 & $<.09$ & .037 \\
\hline
\end{tabular}

\footnotetext{
${ }^{1}$ Minimum of 135 samples (data from Martin and others, 2003).
} 


\section{A. Big Bull Creek watershed}

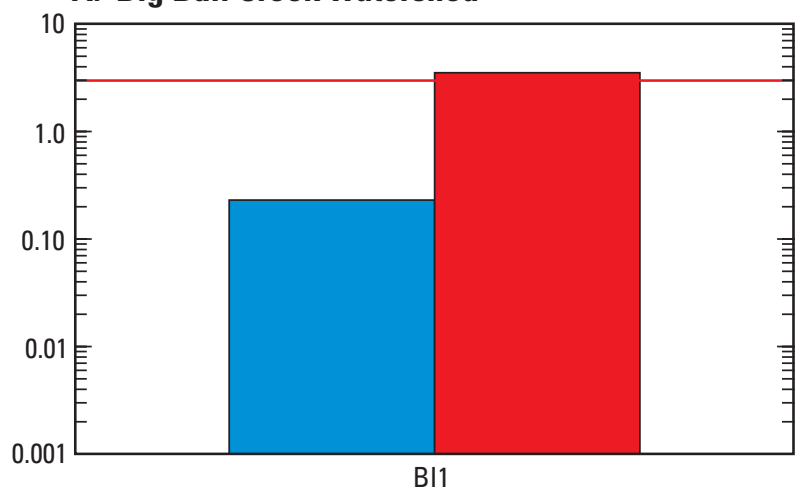

C. Brush Creek watershed

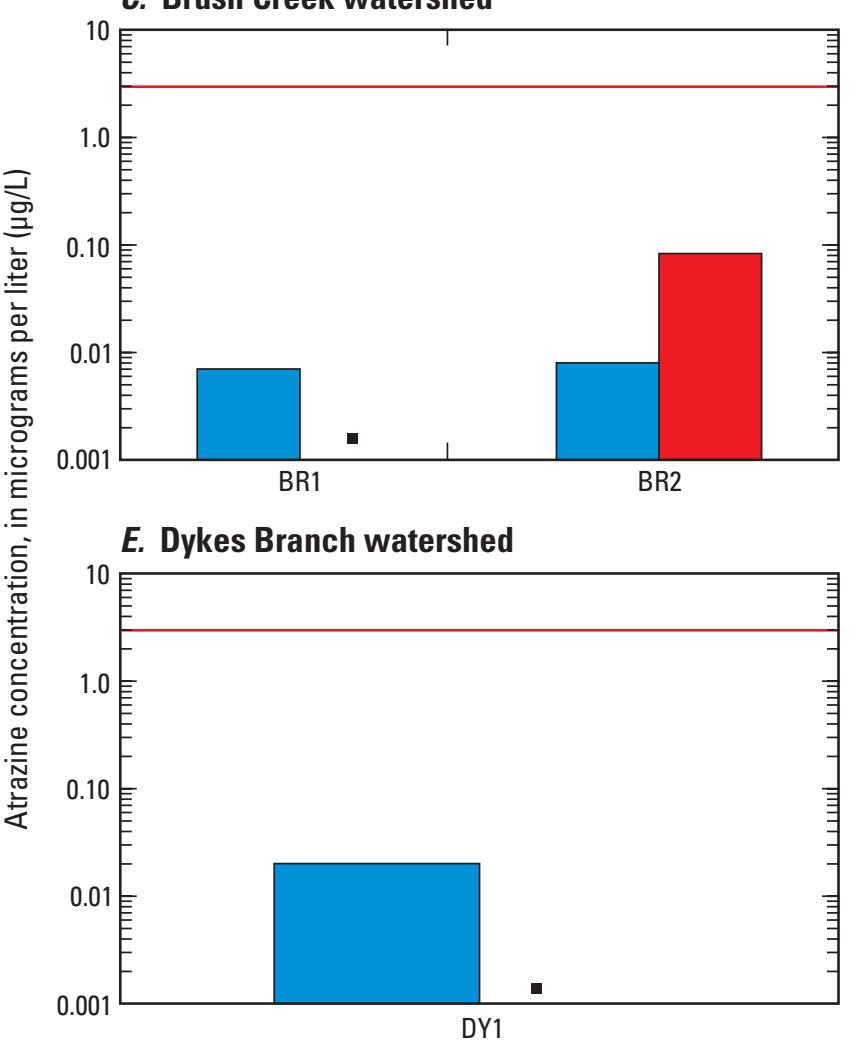

G. Kill Creek watershed

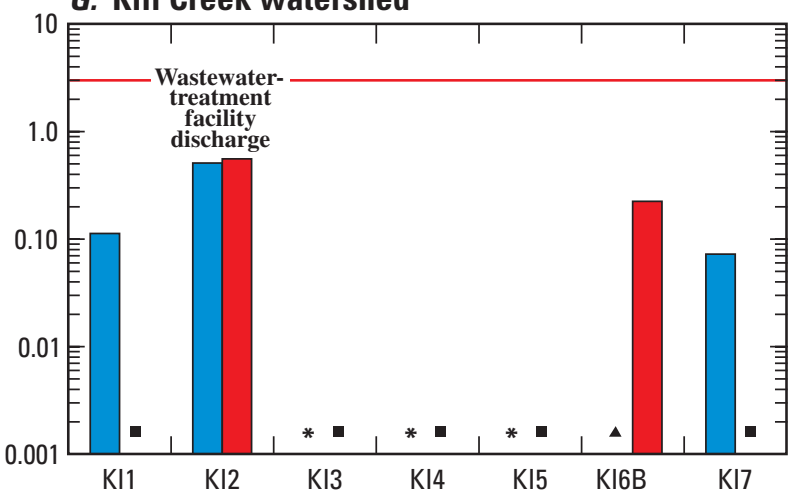

B. Blue River watershed

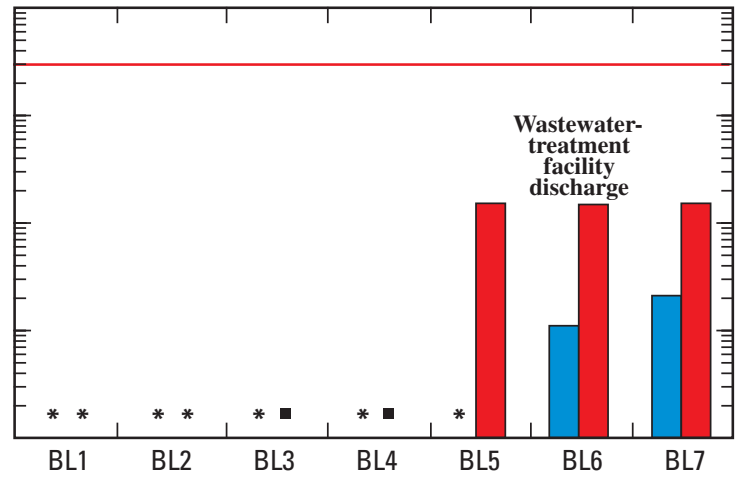

D. Cedar Creek watershed

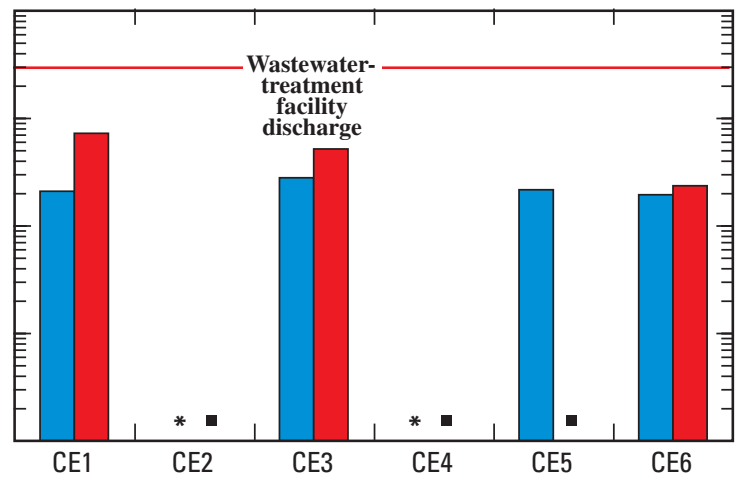

F. Indian Creek watershed

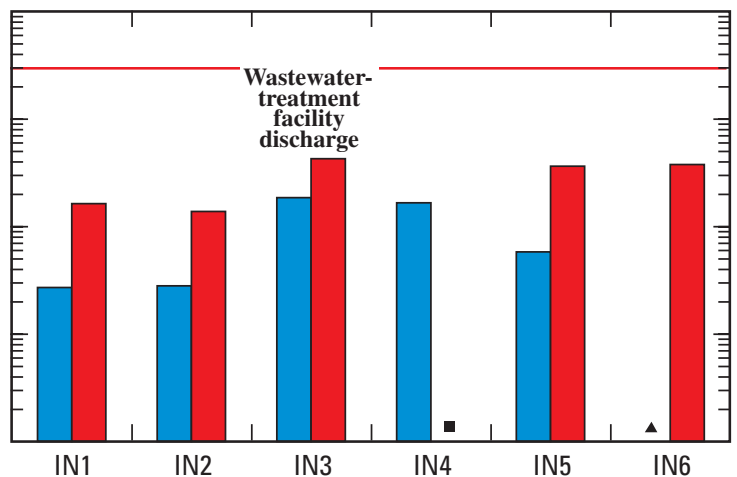

H. Little Bull Creek watershed

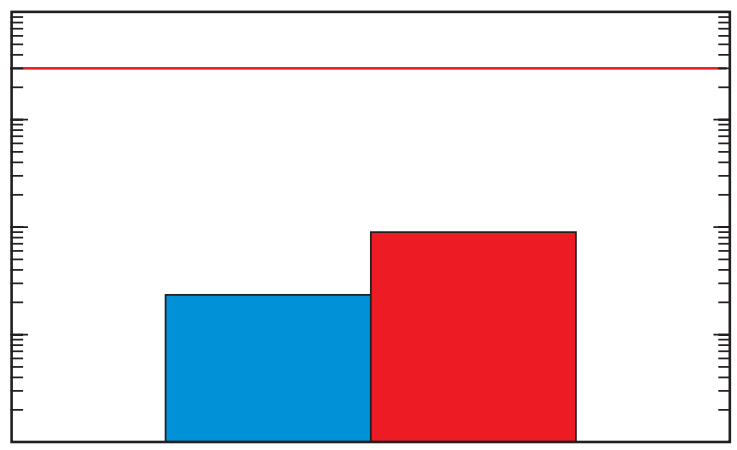

LI1

Sampling site (fig. 1)

Figure 22. Atrazine concentrations in base-flow samples, November 4-7, 2002, and July 14-18, 2003. 


\section{Mill Creek watershed}

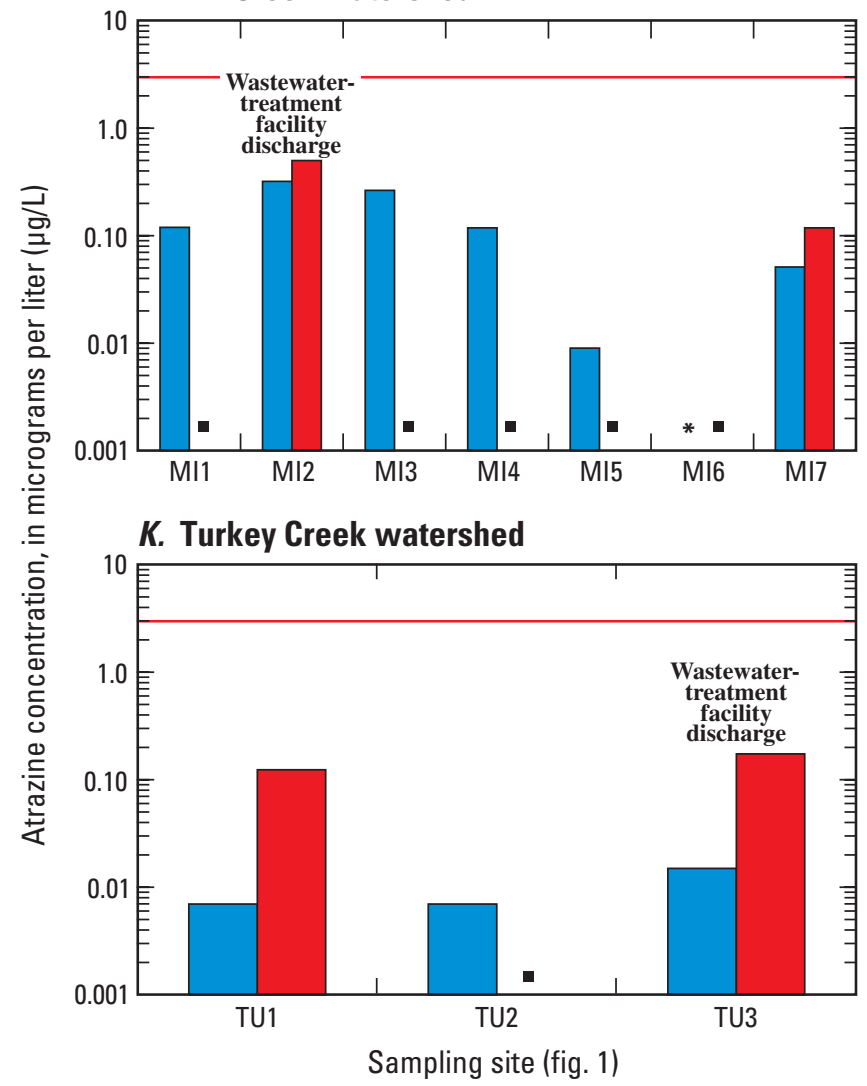

\section{J. Tomahawk Creek watershed}

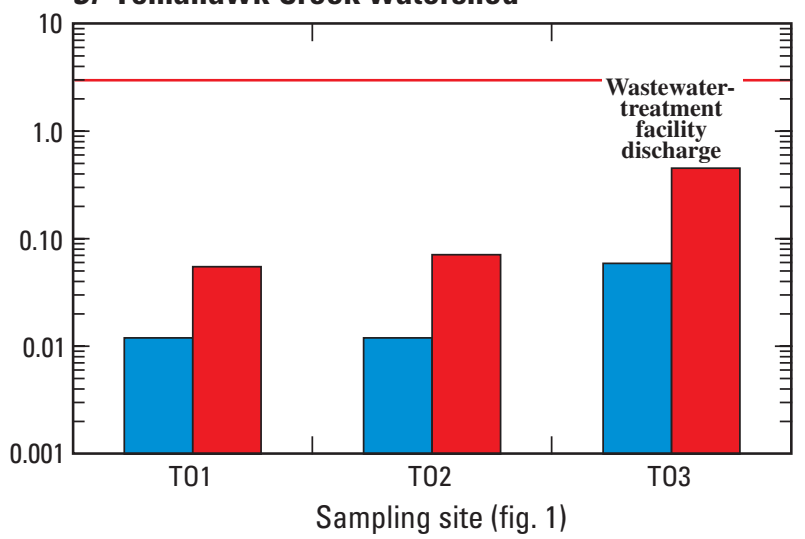

EXPLANATION

Synoptic survey

November 4-7, 2002

July $14-18,2003$

- Kansas Department of Health and Environment (2004c) chronic aquatic-life criterion $(3.0 \mu \mathrm{g} / \mathrm{L})$

* No observable flow-sample not collected

$\Delta$ Site only sampled during second synoptic survey

- Atrazine not analyzed in sample

Figure 22. Atrazine concentrations in base-flow samples, November 4-7, 2002, and July 14-18, 2003.-Continued

samples exceeded $1.0 \mu \mathrm{g} / \mathrm{L}$; all of which were from sites BL5 and KI6b (downstream from predominantly nonurban land uses) collected during May in 2003 and 2004. Acetochlor, metolachlor, and simazine were the only other pesticides detected at larger than microgram-per-liter concentrations in stormflow samples. In comparison to NAWQA median and maximum pesticide concentrations, stormflow samples from Johnson County streams had similar median values (with many nondetections) and smaller maximum values for all detected pesticide compounds (table 18). Large pesticide concentrations in stormflow samples likely are related to sample timing with respect to spring pesticide applications; additional sampling can improve definition of pesticide occurrence in Johnson County streams.

\section{Wastewater-Indicator Compounds}

Wastewater-indicator compounds are categorized as constituents used in commercial, industrial, and residential environments whose sources in freshwater typically are from treated and untreated sewage. Many of these constituents have shown the ability to persist in sewage treatment processes in small (microgram-per-liter) concentrations and discharge into streams. The long-term effects of continuous exposure of humans and animals to different combinations of wastewater compounds currently (2005) are unknown; however, the effects of exposure to some individual constituents have been defined (Daughton and Ternes, 1999).

Fifty-five organic wastewater-indicator compounds were analyzed in stream-water samples collected during base-flow synoptic surveys (tables 6 and 19). Forty-four of these compounds were detected in Johnson County streams. Fifteen compounds were detected at concentrations greater than laboratory reporting levels and are the only compounds discussed in the body of this report.

Every sample collected during base flow, with the exception of a sample from site KI7 collected during the November 2002 survey, contained at least one wastewater compound (fig. 24A). Samples with the most wastewater compounds detected were either from WWTF discharge sites or from sites immediately downstream from WWTFs. Acetyl-hexamethyltetrahydro-napthalene (AHTN), caffeine, DEET, nonylphenoldiethoxylate, and tris(2-butoxyethyl) phosphate made up greater than 50 percent of the total wastewater compound concentrations in 59 of 73 base-flow samples.

The largest total concentrations of wastewater compounds (fig. 24B) were in base-flow samples from sites at or immediately downstream from trickling-filter WWTF discharges (sites MI2, TO3, TU3; table 3). Upstream from WWTF discharges, a Mann-Whitney test of independent groups showed that base-flow samples from sites with mostly urban watersheds 

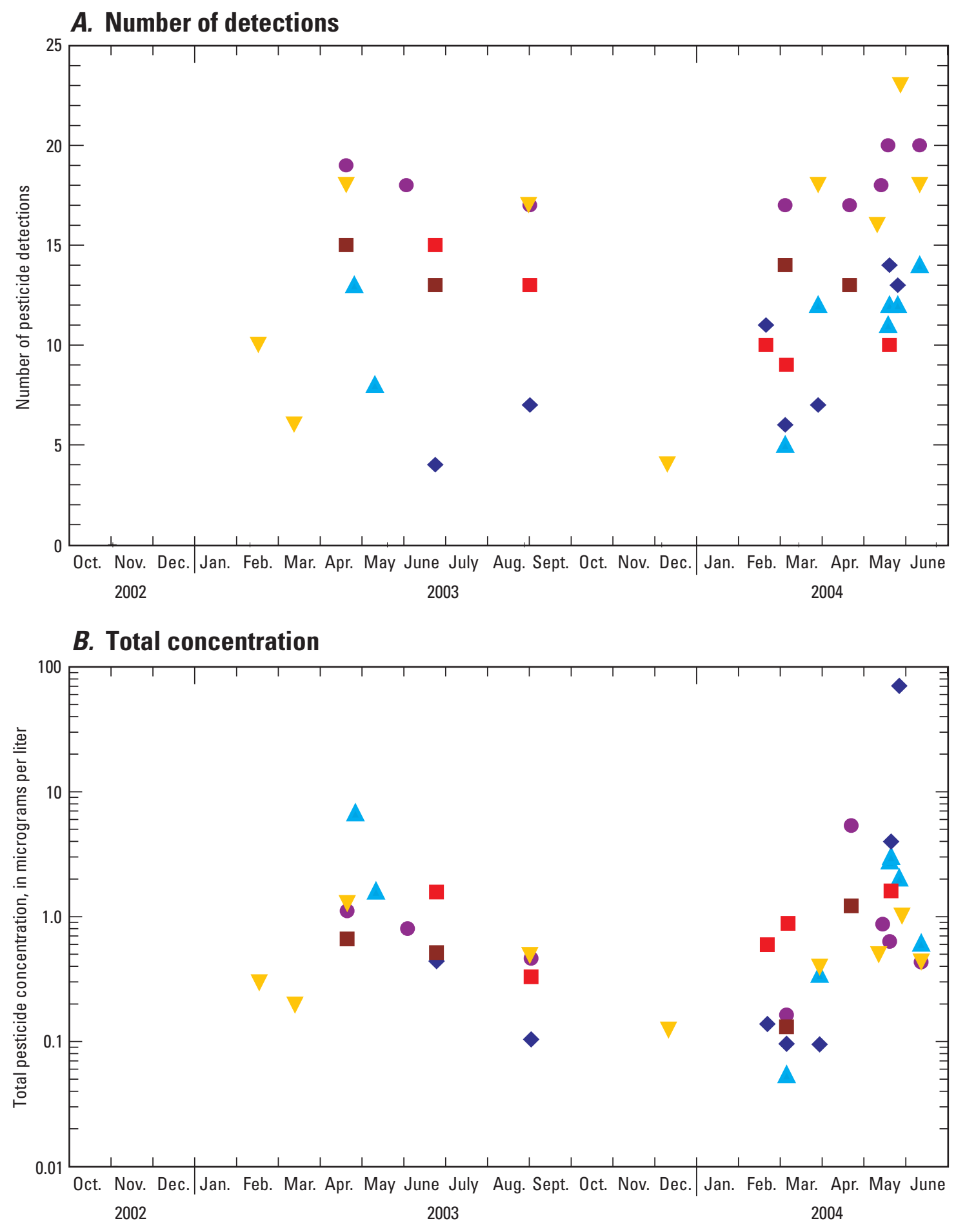

\section{EXPLANATION}

\section{Stormflow sampling} sites (fig. 1)

$$
\begin{array}{ll}
\Delta \text { BL5 } & \bullet \text { KI6b } \\
\text { - CE6 } & \nabla \text { MI7 } \\
- \text { IN6 } & \text { - TU1 }
\end{array}
$$

Figure 23. (A) Number of pesticide compound detections and $(B)$ total concentrations in stormflow samples, February 2003-June 2004. 
Table 19. Organic wastewater-indicator compounds analyzed in stream-water samples from selected streams in Johnson County, northeastern Kansas, October 2002-June 2004.

[PAH, polycyclic aromatic hydrocarbon]

\begin{tabular}{|c|c|}
\hline Compound & Possible compound uses or sources $^{1}$ \\
\hline 1,4-Dichlorobenzene & Moth repellent, fumigant, deodorant metabolite \\
\hline 1-Methylnaphthalene & 2-5 percent of gasoline, diesel fuel, or crude oil \\
\hline 2,6-Dimethylnaphthalene & Present in diesel/kerosene (trace in gasoline) \\
\hline 2-Methylnaphthalene & 2-5 percent of gasoline, diesel fuel, or crude oil \\
\hline 3-Beta-coprostanol & Carnivore fecal indicator \\
\hline 3-Methyl-1(H)-indole (Skatol) & Fragrance, stench in feces and coal tar \\
\hline 3-Tert-Butyl-4-hydroxy anisole (BHA) & Antioxidant, general preservative \\
\hline 4-Cumylphenol & Nonionic detergent metabolite \\
\hline 4-Nonylphenol & Nonionic detergent metabolite \\
\hline 4-Octylphenol & Nonionic detergent metabolite \\
\hline 4-Tert-octylphenol & Nonionic detergent metabolite \\
\hline 5-Methyl-1H-benzotriazole & Antioxidant in antifreeze and deicers \\
\hline 9,10-Anthraquinone & Bird repellent, manufactured dye/textiles, seed treatment \\
\hline Acetophenone & Fragrance in detergent and tobacco, flavor in beverages \\
\hline Acetyl-hexamethyl-tetrahydro-naphthalene & Musk fragrance (widespread usage), persistent in ground water \\
\hline Anthracene & Wood preservative, component of tar, diesel, or crude oil, combustion product \\
\hline Benzo A Pyrene & Regulated PAH, used in cancer research, combustion product \\
\hline Benzophenone & Fixative for perfumes and soaps \\
\hline beta-Sitosterol & Plant sterol \\
\hline beta-Stigmastanol & Plant sterol \\
\hline Bisphenol A & Manufactured polycarbonate resins, antioxidant, flame retardant \\
\hline Bromacil & Herbicide, pesticide, greater than 80 percent noncrop usage \\
\hline Caffeine & Beverages, diuretic, very mobile/biodegradable \\
\hline Camphor & Flavor, odorant, ointments \\
\hline Carbazole & Component of coal tar, oil, petroleum products \\
\hline Cholesterol & Often a fecal indicator, plant sterol \\
\hline Cotinine & Primary nicotine metabolite \\
\hline DEET (N,N-diethyl-meta-toluamide) & Insecticide, urban uses, mosquito repellent \\
\hline d-Limonene & Fungicide, antimicrobial, antiviral, fragrance \\
\hline Fluoranthene & Component of coal tar and asphalt, combustion product \\
\hline $\begin{array}{l}\text { Hexahydro-hexamethyl-cyclopentabenzopyran } \\
\text { (HHCB) }\end{array}$ & Musk fragrance (widespread usage), persistent in ground water \\
\hline Indole & Pesticide inert ingredient, fragrance in coffee \\
\hline Isoborneol & Fragrance in perfumery, disinfectants \\
\hline Isophorone & Fragrance in perfumery, disinfectants \\
\hline Isopropyl benzene (Cumene) & Manufactured phenol/acetone, fuels, and paint thinner \\
\hline
\end{tabular}


Table 19. Organic wastewater-indicator compounds analyzed in stream-water samples from selected streams in Johnson County, northeastern Kansas, October 2002-June 2004.-Continued

[PAH, polycyclic aromatic hydrocarbon]

\begin{tabular}{|c|c|}
\hline Compound & Possible compound uses or sources ${ }^{1}$ \\
\hline Isoquinonline & Flavors and fragrances \\
\hline Menthol & Cigarettes, cough drops, liniment, mouthwash \\
\hline Methyl salicylate & Liniment, food, beverage, ultraviolet-absorbing lotion \\
\hline Naphthalene & Fumigant, moth repellent, major component of gasoline \\
\hline Nonylphenol- diethoxylate & Nonionic detergent metabolite \\
\hline Octylphenol-diethoxylate & Nonionic detergent metabolite \\
\hline Octylphenol-ethoxylate & Nonionic detergent metabolite \\
\hline Para-Cresol & Wood preservative \\
\hline Pentachlorophenol & Herbicide, fungicide, wood preservative, termite control \\
\hline Phenanthrene & $\begin{array}{l}\text { Manufactured explosives, component of tar, diesel fuel, crude oil, combustion } \\
\text { byproduct }\end{array}$ \\
\hline Phenol & Disinfectant, manufacture of several products, leachate \\
\hline Pyrene & $\begin{array}{l}\text { Component of coal tar and asphalt (only traces in gasoline or diesel fuel), } \\
\text { combustion product }\end{array}$ \\
\hline Tetrachloroethylene & Solvent, degreaser, veterinary anthelminitic \\
\hline Tributylphosphate & Antifoaming agent, flame retardant \\
\hline Triclosan & Disinfectant, antimicrobial \\
\hline Triethyl citrate & Cosmetics, pharmaceuticals \\
\hline Triphenyl phosphate & Plasticizer, resin, wax, finish, roofing paper, flame retardant \\
\hline Tris(2-butoxyethyl) phosphate (TBEP) & Plasticizer, floor polish, flame retardant \\
\hline Tris(2-chloroethyl) phosphate (Fyrol CEF) & Flame retardant, plasticizer \\
\hline Tris(dichlorisopropyl) phosphate (Fyrol PCF) & Flame retardant \\
\hline
\end{tabular}

(sites BR1, BR2, DY1, IN1, IN2, MI1, TO1, TO2, TU1, TU2; table 4; fig. 2) had significantly larger ( $p$ value less than 0.05 ) median concentrations $(6.1 \mu \mathrm{g} / \mathrm{L})$ of wastewater compounds than samples from sites in mostly nonurban watersheds (sites BL3, BL4, BL5, CE1, CE2, KI1, MI5, MI6) (2.4 $\mu \mathrm{g} / \mathrm{L})$. These larger concentrations indicate that additional sources (other than WWTFs) of wastewater compounds exist in urban watersheds. These sources could range from leaking sewage lines, illicit discharges, atmospheric deposition, ground water, or interaction between stream water and streambed sediment.

Concentrations in Johnson County stream-water samples were compared to data from USGS studies performed by Kolpin and others (2002) and Lee and others (2004). Both of these studies analyzed wastewater compounds and pharmaceuticals from whole-water samples (this study used filtered water samples). Lee and others (2004) observed that whole- and filtered-water analyses found similar concentrations for most wastewater and pharmaceutical compounds in a Minnesota study; however, some compounds had smaller concentrations in filtered-water analyses due to adsorption to suspended sediment. Thus, values from these studies may have larger concentrations of some contaminants due to the inclusion of sediment-bound and dissolved contaminants. In addition, each of these studies had different sampling designs. Kolpin and others (2002) did not directly sample WWTF discharges; thus, concentrations in the Johnson County study reported herein may be larger due to the direct sampling of WWTF discharges. Lee and others (2004) sampled wastewater compounds collected in WWTF influent, effluent, landfill and feedlot lagoon leachate, surface water, ground water, and WWTF discharges. Concentrations from these samples may be larger than concentrations in the Johnson County study due to the sampling of raw sewage.

Several of the compounds in Johnson County base-flow samples (table 20) were detected at larger maximum concentrations than those reported in a nationwide reconnaissance of pharmaceuticals, hormones, and other organic wastewaterindicator contaminants conducted in 1999-2000 (Kolpin and others, 2002). Although Lee and others (2004) sampled raw sewage, the compounds caffeine, nonylphenol-diethoxylate, and tris(2-butoxyethyl) phosphate were detected at larger 


\section{A. Number of detections}

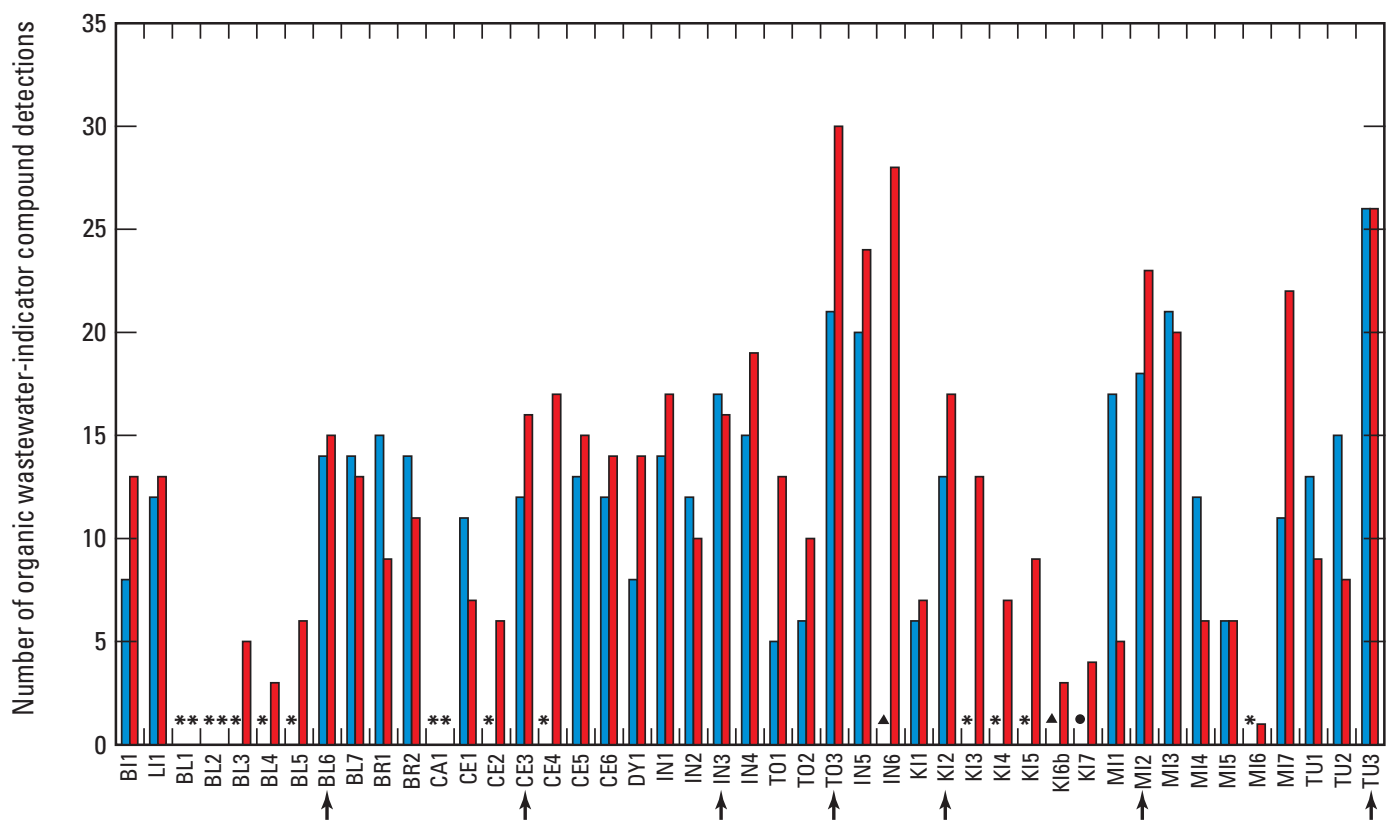

B. Total concentration

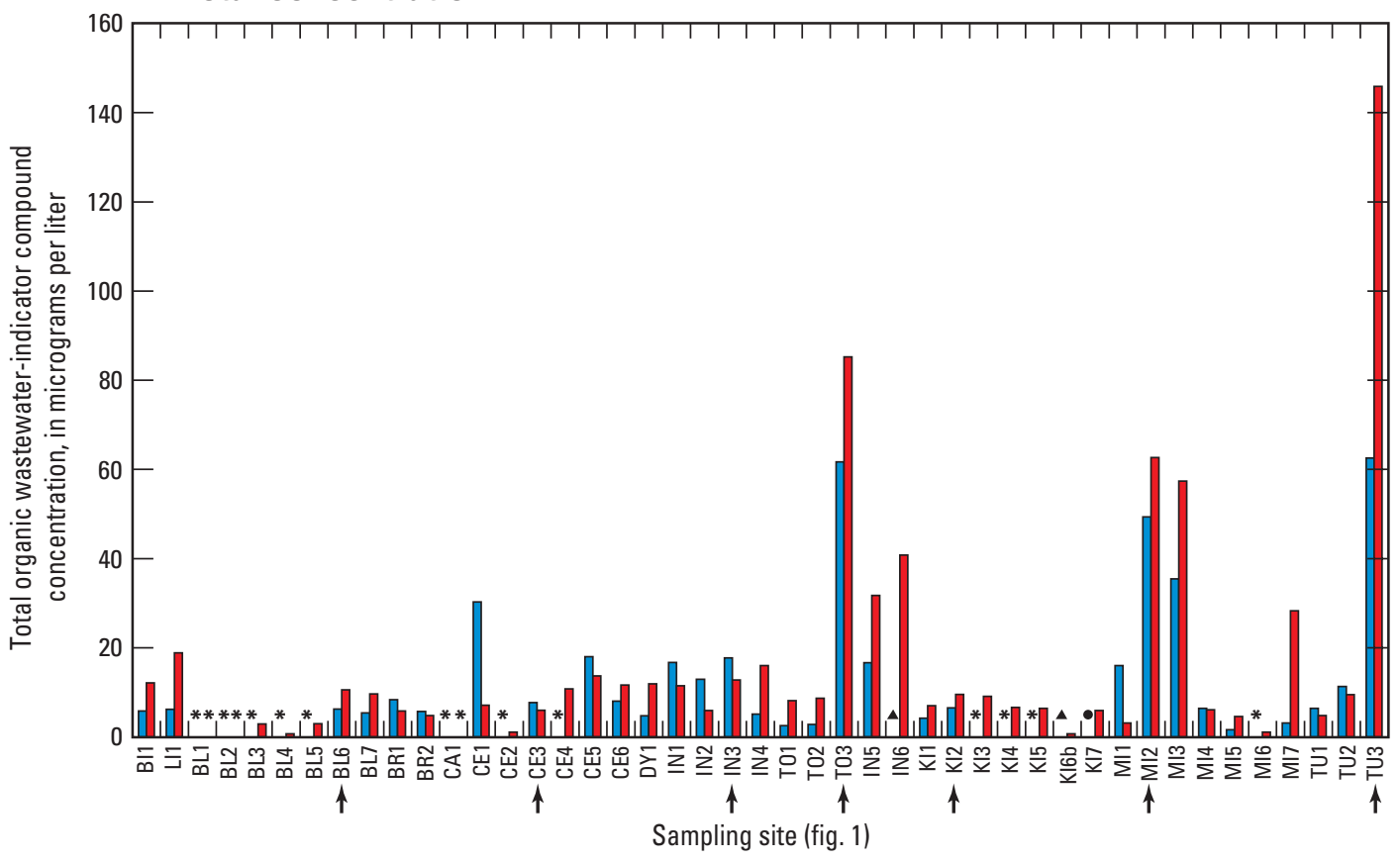

EXPLANATION

Synoptic survey

November 4-7, 2002

July $14-18,2003$

$\uparrow$ Wastewater-treatment facility discharge

* No observable flow-Sample not collected

$\triangle \quad$ Site only sampled during second synoptic survey

- No detection of organic wastewater-indicator compounds

Figure 24. (A) Number of organic wastewater-indicator compound detections and (B) total concentrations in base-flow samples, November 4-7, 2002, and July 14-18, 2003. 
Table 20. Median and maximum concentrations of organic wastewater-indicator compounds detected at concentrations larger than laboratory reporting levels in base-flow samples from selected Johnson County streams, northeastern Kansas, November 4-7, 2002, and July 14-18, 2003, and samples collected nationwide by the U.S. Geological Survey (Kolpin and others, 2002), 1999-2000, and in Minnesota (Lee and others, 2004), 2000-02.

$[<$, less than; E, estimated; --, not analyzed]

\begin{tabular}{|c|c|c|c|c|c|c|c|c|}
\hline \multirow[b]{2}{*}{ Wastewater compound } & \multirow[b]{2}{*}{ Category } & \multirow[b]{2}{*}{$\begin{array}{l}\text { Number of } \\
\text { samples }\end{array}$} & \multirow[b]{2}{*}{$\begin{array}{l}\text { Detection } \\
\text { frequency } \\
\text { (percent) }\end{array}$} & \multicolumn{5}{|c|}{ Concentration (micrograms per liter) } \\
\hline & & & & $\begin{array}{l}\text { Median } \\
\text { Johnson } \\
\text { County } \\
\text { study }^{1}\end{array}$ & $\begin{array}{l}\text { Maximum Johnson County } \\
\text { study }^{1} \text { (sampling site } \\
\text { identifier, fig. 1) }\end{array}$ & $\begin{array}{l}\text { Median from } \\
\text { Kolpin and } \\
\text { others }{ }^{2} \\
\text { (2002) }\end{array}$ & $\begin{array}{l}\text { Maximum } \\
\text { from Kolpin } \\
\text { and others }{ }^{2} \\
\text { (2002) }\end{array}$ & $\begin{array}{l}\text { Maximum } \\
\text { from Lee and } \\
\text { others }{ }^{3} \\
\text { (2004) }\end{array}$ \\
\hline 3-beta-coprostanol & fecal indicator & 73 & 14 & $<1$ & $5.0(\mathrm{TO} 3, \mathrm{TU} 3)$ & 0.7 & 9.8 & 81 \\
\hline 4-nonylphenol (4-NP) & detergent metabolite & 73 & 59 & $<5$ & E17 (TU3) & .8 & 40 & 56 \\
\hline $\begin{array}{l}\text { Acetyl-hexamethyl-tetrahydro- } \\
\text { napthalene (AHTN) }\end{array}$ & fragrance & 73 & 52 & $<.5$ & 2.3 (MI2) & -- & -- & 5.3 \\
\hline beta-Sitosterol & plant sterol & 73 & 27 & $<2$ & 3.0 (IN6, MI7, TO3) & -- & -- & 36 \\
\hline beta-Stigmastanol & plant sterol & 73 & 57 & $<2$ & 4.0 (MI7) & 2.0 & 4.0 & 5.7 \\
\hline Caffeine & diuretic & 73 & 78 & $<.5$ & 12 (TO3) & .1 & 5.7 & .5 \\
\hline Cholesterol & fecal indicator, plant sterol & 73 & 36 & $<2$ & 10 (TO3) & 1 & 10 & 130 \\
\hline Menthol & additive in cigarettes & 73 & 15 & $<.5$ & $1.4(\mathrm{TO} 3)$ & -- & -- & 96 \\
\hline $\begin{array}{l}\text { N,N-diethyl-meta- } \\
\text { toluamide (DEET) }\end{array}$ & insecticide & 73 & 88 & $<.5$ & 3.7 (TU3) & .1 & 1.1 & 47 \\
\hline $\begin{array}{l}\text { Nonylphenol-diethoxylate } \\
\text { (NPEO2) }\end{array}$ & detergent surfactant & 73 & 90 & $<5$ & 63 (TU3) & 1 & 9 & 42 \\
\hline Phenol & disinfectant & 73 & 33 & $<.5$ & $1.8(\mathrm{TO} 3)$ & E.7 & E1.3 & -- \\
\hline Triclosan & disinfectant, antimicrobial & 73 & 5 & $<1$ & $2.0(\mathrm{TO} 3, \mathrm{TU} 3))$ & .1 & 2.3 & 4.3 \\
\hline $\begin{array}{l}\text { Tris(2-butoxyethyl) } \\
\text { phosphate (TBEP) }\end{array}$ & $\begin{array}{l}\text { flame retardant, floor } \\
\text { polish }\end{array}$ & 73 & 66 & $<.5$ & 27 (TU3) & .5 & 6.7 & 5.3 \\
\hline Tris(2-chloroethyl) phosphate & flame retardant, plasticizer & 73 & 64 & $<.5$ & $.6(\mathrm{BL} 6, \mathrm{IN} 3, \mathrm{MI} 2, \mathrm{MI} 3)$ & .1 & .5 & 9.2 \\
\hline Tris(dichloroisopropyl) phosphate & flame retardant & 73 & 64 & $<.5$ & $.6(\mathrm{IN} 3)$ & .1 & .2 & 2.5 \\
\hline
\end{tabular}

\footnotetext{
${ }^{1}$ Analyzed filtered water samples.

${ }^{2}$ Analyzed whole water samples.

${ }^{3}$ Analyzed whole and filtered water samples.
} 
maximum concentrations in samples from selected Johnson County trickling-filter WWTF discharges (sites TO3 and TU3). Several of the wastewater compounds were not detected down stream from WWTF discharges in Johnson County, indicating adsorption to sediment, biodegradation, and (or) degradation to metabolites not analyzed in this study.

Thirty-six of the 55 wastewater compounds analyzed were detected in stormflow samples; however, only 10 of 55 were detected at concentrations larger than laboratory reporting levels. Samples from urban sites (site TU1, 20 detections; site IN6, 18 detections) averaged the most detections of wastewater compounds, whereas samples from nonurban site KI6b averaged the least (4) (fig. 25).

The wastewater compounds that were detected at concentrations larger than laboratory reporting levels in stormflow samples generally had smaller concentrations compared to base-flow samples, likely due to dilution of WWTF point sources. Caffeine, DEET, and nonylphenol-diethoxylate (NPEO2) were the only compounds detected in samples from the majority of stormflow sites, and 9, 10-anthraquinone, caffeine, carbazole, NPEO2, and tris(2-butoxyethyl) phosphate were the only wastewater compounds detected at concentrations larger than laboratory reporting levels and larger than microgram-per-liter levels. 9,10-anthraquinone and carbazole were detected in the largest concentrations (of all stream-water samples) in stormflow samples from site TU1 on April 20, 2004. These concentrations were also larger than maximum detections found by Kolpin and others (2002) and Lee and others (2004).

Wastewater compounds analyzed in Johnson County stream water can be broadly categorized as detergent metabolites, flame retardants and plasticizers, fragrances, caffeine, insecticides/bird repellents/petroleum compounds, and sterols and stanols. Each of these categories are discussed in the following sections.

\section{Detergent Metabolites}

Alkylphenol ethoxylates (APEOs) are a class of surfactants used in commercial, industrial, and residential applications. Octylphenol ethoxylates (OPEOs) and nonylphenol ethoxylates (NPEOs) are two of the most common surfactants in the marketplace and are used in domestic detergents, pesticide formulations, and in industrial products (Ying and others, 2002). NPEOs comprise 80 percent of the worldwide market, whereas OPEOs comprise 20 percent (White and others, 1994). NPEOs were the only detergent metabolites detected at greater than laboratory reporting levels in Johnson County streams and thus are the only APEOs discussed in this report.

Degradation of NPEOs in WWTFs or in the environment often results in formation of shorter chain compounds such as nonylphenol mono-, di-, and triethoxylate and 4-nonylphenol (4-NP) (Giger and others, 1984). Shorter chain compounds such as 4-NP are typically the most persistent, toxic, and estrogenic compounds of the detergent metabolites (Routedge and Sumpter, 1997). 4-NP has been found to cause estrogenic effects in fish such as increasing vitellogenin production and inhibiting testicular growth in male fish (Soto and others, 1991; Jobling and Sumpter, 1993; White and others, 1994; Jobling and others, 1996). USEPA has released a draft criteria on the occurrence of 4-NP in freshwater. These criteria establish acute toxicity $(55.7 \mu \mathrm{g} / \mathrm{L})$ and chronic toxicity $(5.9 \mu \mathrm{g} / \mathrm{L})$ guidelines for 4-NP in freshwater (U.S. Environmental Protection Agency, 2003). The acute toxicity criterion describes the concentration below which 4-NP should not be lethal; the chronic toxicity criterion describes the concentration below which most species should not be affected (U.S. Environmental Protection Agency, 2003).

NPEO2 and 4-NP concentrations were largest in samples from the effluent of trickling-filter WWTFs (sites MI2, TO3, TU3; table 3), and the largest NPEO2 and 4-NP concentrations were in samples collected during the July 2003 synoptic survey in the Turkey Creek watershed at WWTF discharge site TU3 (63 and $17 \mu \mathrm{g} / \mathrm{L}$, respectively) (table 20). The maximum NPEO2 concentration ( $63 \mu \mathrm{g} / \mathrm{L}$ at site TU3; fig. 26) was larger than any detected by Kolpin and others (2002) or by Lee and others (2004). This large concentration may due to large influent concentrations levels, poor removal by trickling-filter treatment processes, and (or) poor removal due to individual treatment facility characteristics. Trickling-filter WWTFs have been shown to be less efficient in the removal of detergent metabolites (Phillips and others, 2004).

NPEO2 concentrations exceeded 4-NP concentrations in all base-flow samples, with more frequent detections and larger maximum values. NPEO2 was detected in 66 of 73 samples, up- and downstream from WWTF discharges and in urban and nonurban areas. 4-NP concentrations exceeded USEPA draft chronic toxicity criterion $(5.9 \mu \mathrm{g} / \mathrm{L})$ in five samples; all either were collected at or downstream from WWTF sites MI2 or TU3. NPEO2 and 4-NP concentrations decreased downstream from WWTF discharges. NPEO metabolites have large octanol-water partitioning coefficients $\left(\log \mathrm{K}_{\mathrm{ow}}\right.$ from 4.0 to 4.5 ), indicating that they are likely to partition to sediment following discharge from WWTFs (Ying and others, 2002). Rapid loss of NPEO2 and 4-NP downstream from sources suggests degradation to metabolites and (or) adsorption from stream water to streambed sediment.

Other than in base-flow WWTF discharge samples, NPEO2 concentrations in stormflow samples were the largest during spring 2004 (fig. 27). A sample collected on March 4, 2004, from site TU1 (upstream from WWTF discharges) had the largest NPEO2 concentration $(51 \mu \mathrm{g} / \mathrm{L})$ in stormflow samples. Although concentrations of NPEO2 were not significantly different in base- and stormflow samples ( $p$ value $=0.08$ ), estimated hourly loads of stormflow samples were significantly larger ( $p$ value less than 0.01) (fig. 28). Large NPEO2 concentrations and loads in stormflow samples indicate that there were significant nonpoint sources of these compounds. NPEOs are used in agricultural pesticides; however, large concentrations of pesticides were not found in the samples in conjunction with large NPEO2 concentrations. It is possible that large surfactant concentrations could have been related to sources from septic 


\section{A. Number of detections}
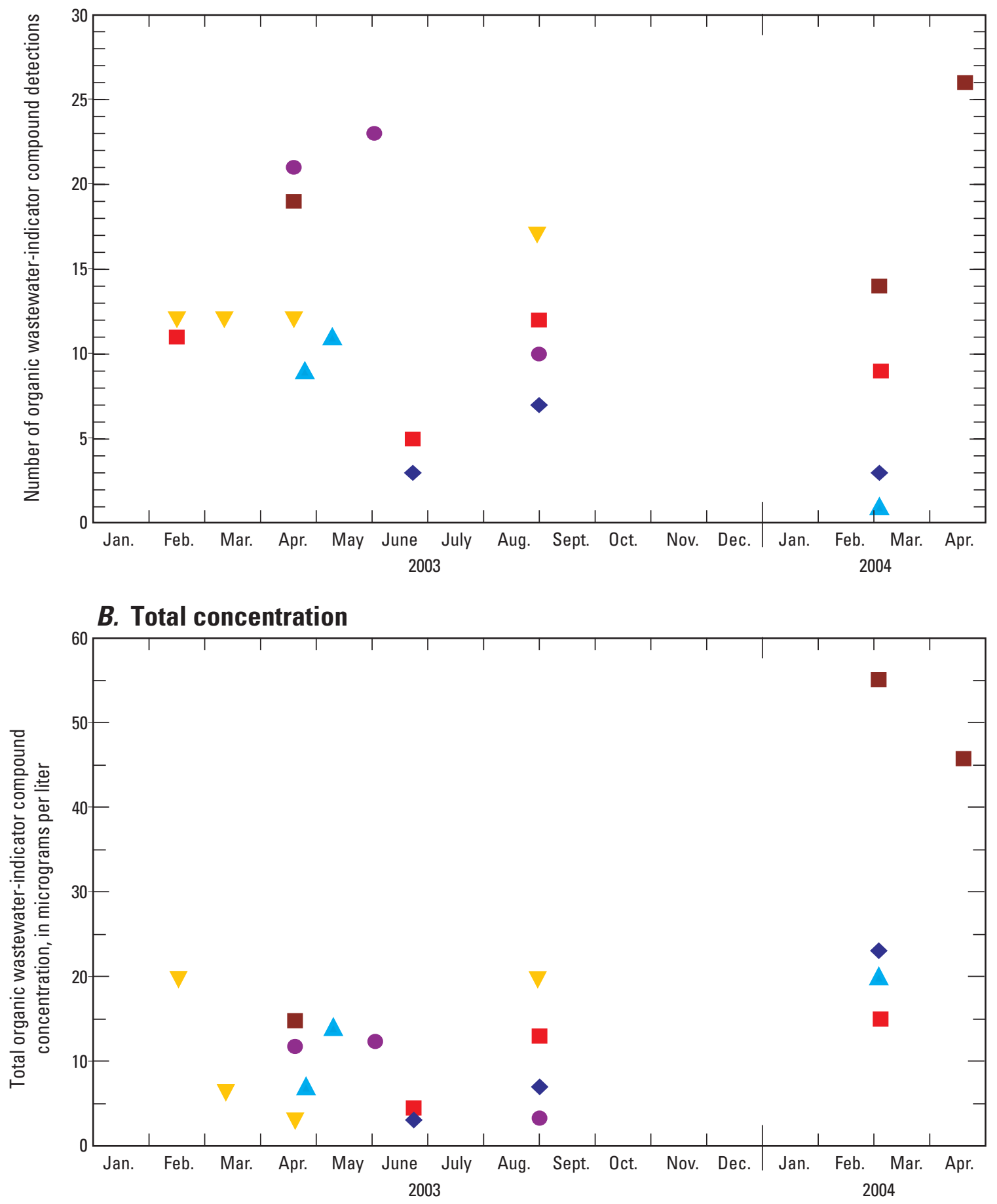

EXPLANATION

Stormflow sampling sites (fig. 1)

$$
\begin{array}{ll}
\triangle \text { BL5 } & \diamond \text { KI6b } \\
\text { - CE6 } & \nabla \text { MI7 } \\
\text { - IN6 } & \square \text { TU1 }
\end{array}
$$

Figure 25. (A) Number of organic wastewater-indicator compound detections and $(B)$ total compound concentrations in stormflow samples, February 2003-April 2004. 
A. Big Bull Creek watershed

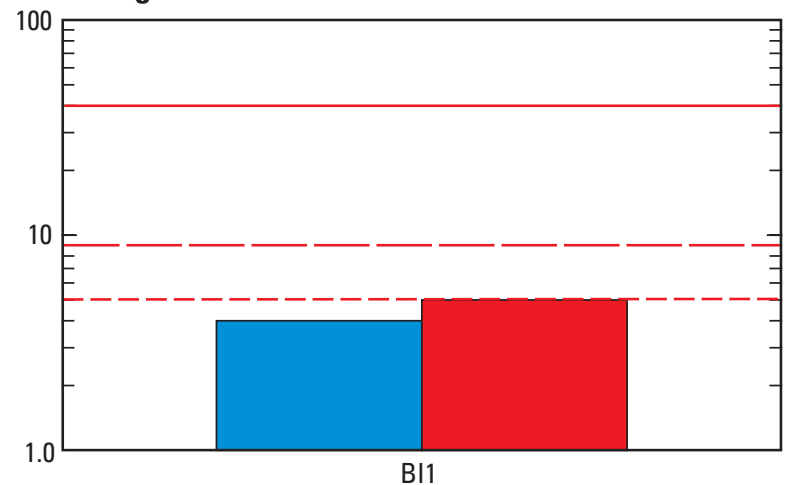

C. Brush Creek watershed

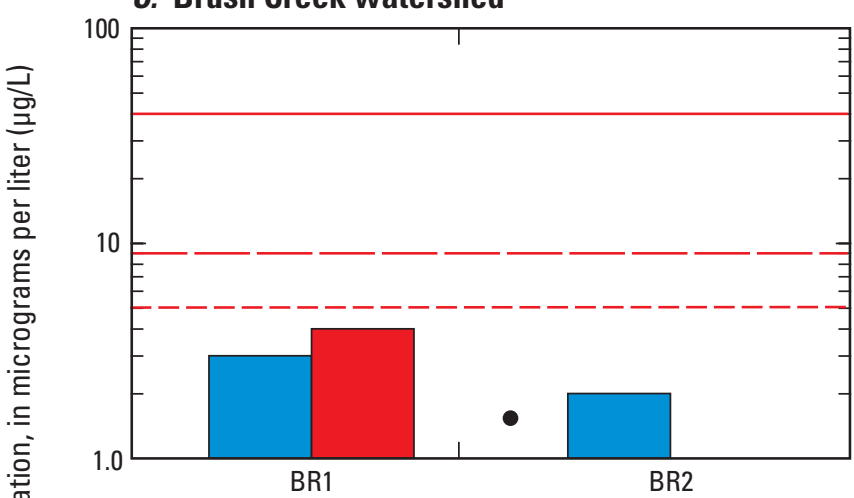

E. Dykes Branch watershed

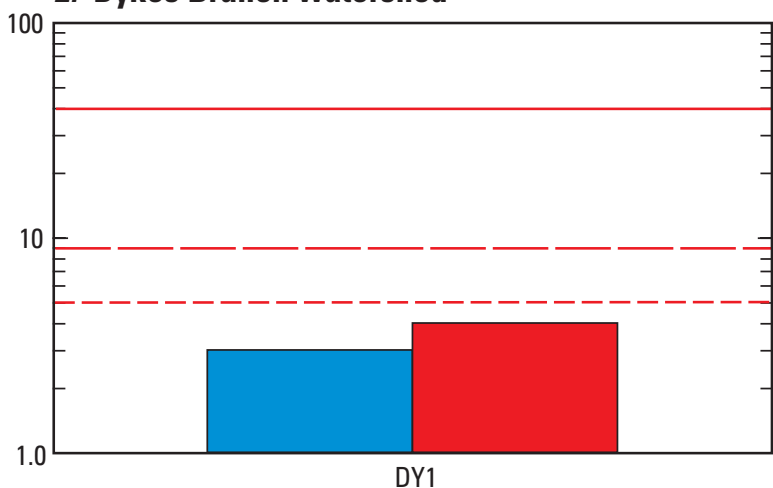

G. Kill Creek watershed

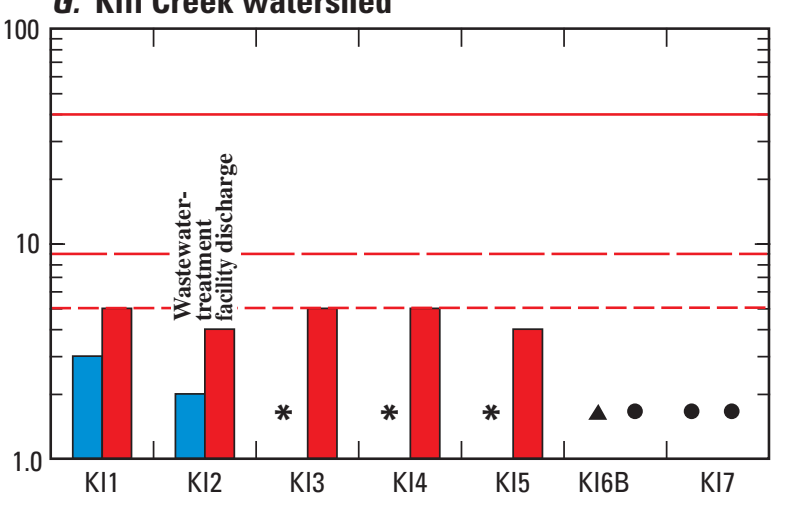

B. Blue River watershed

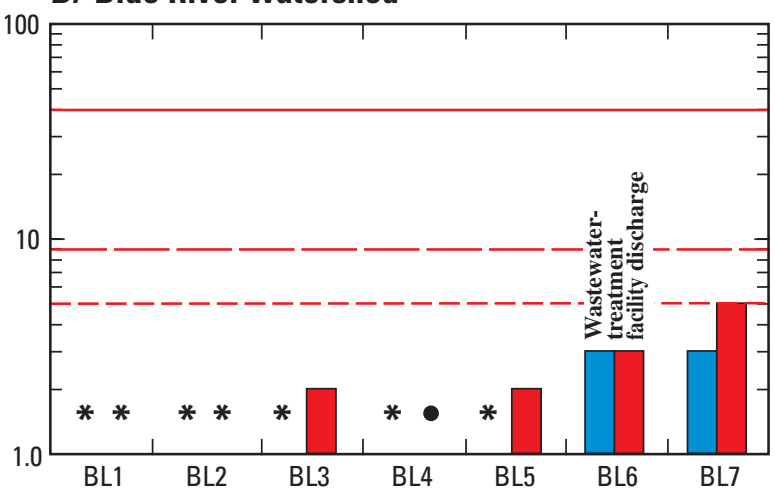

D. Cedar Creek watershed

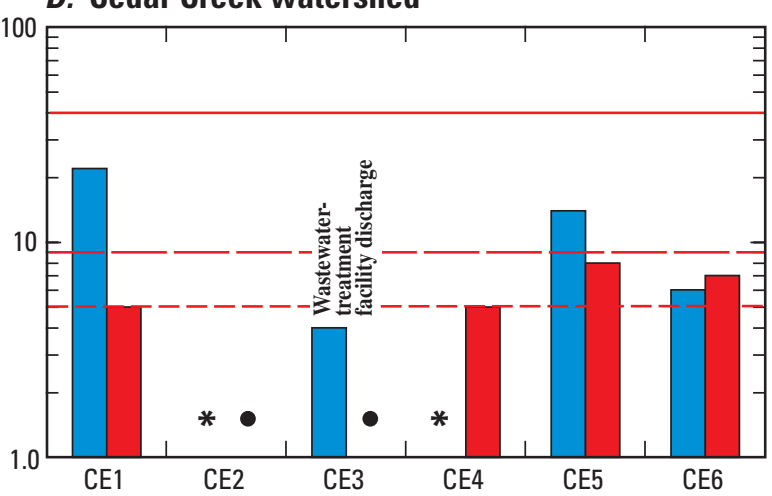

F. Indian Creek watershed
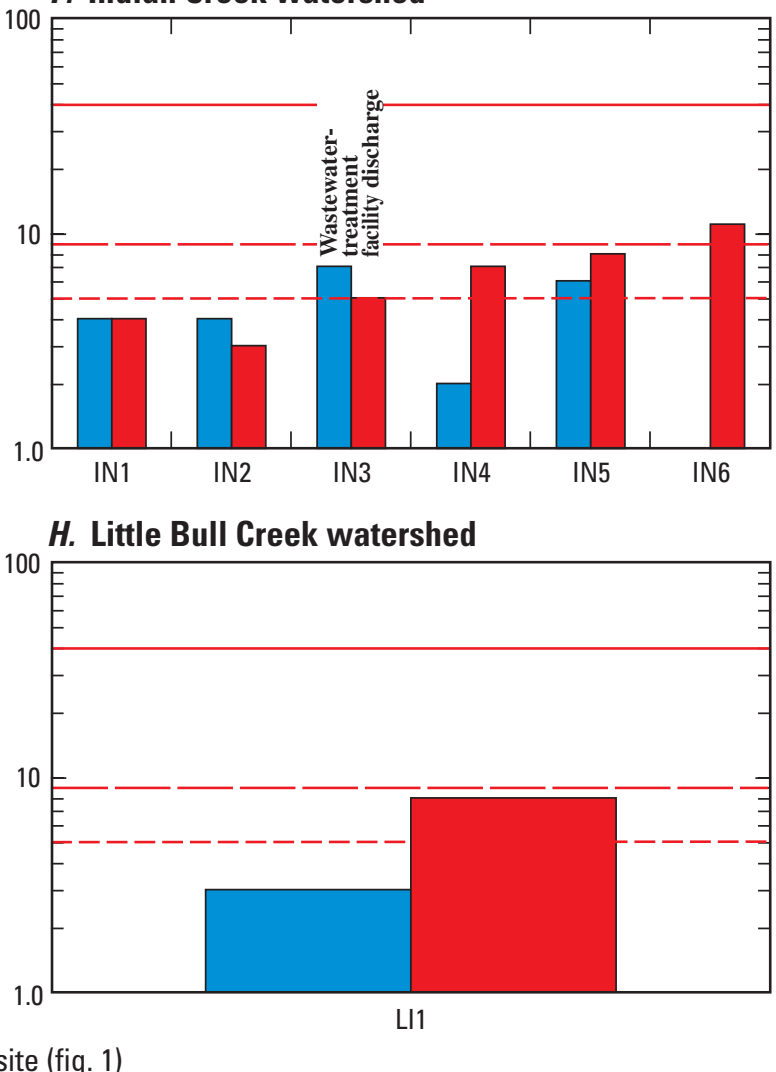

Figure 26. Nonylphenol-diethoxylate concentrations in base-flow samples, November 4-7, 2002, and July 14-18, 2003. 

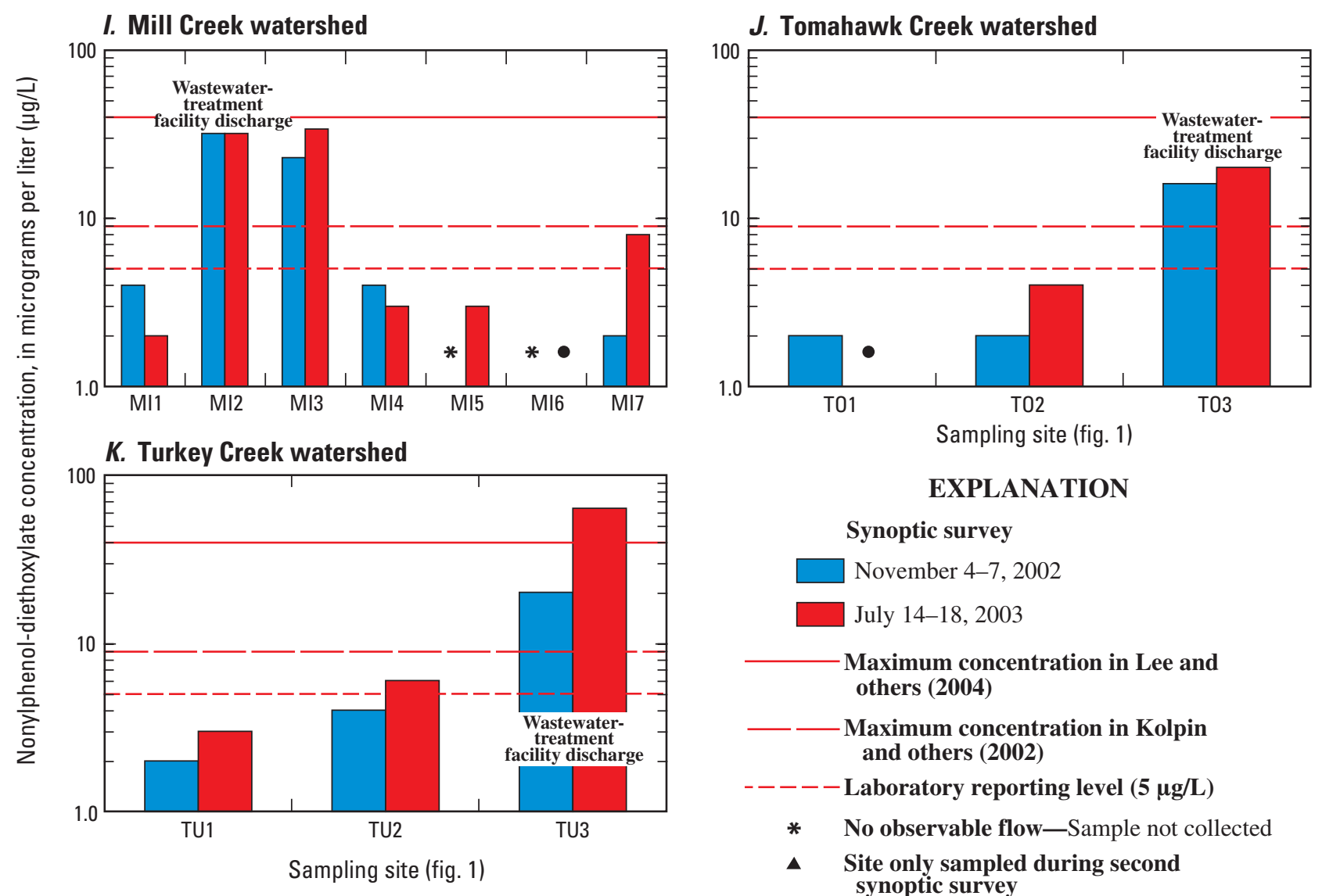

\section{EXPLANATION}

Synoptic survey

November 4-7, 2002

$\square$ July 14-18, 2003

Maximum concentration in Lee and others (2004)

- Maximum concentration in Kolpin and others (2002)

----- Laboratory reporting level $(5 \mu \mathrm{g} / \mathrm{L})$

* No observable flow-Sample not collected

\ Site only sampled during second synoptic survey

- No detections of nonylphenoldiethoxylate (NPE02)

Figure 26. Nonylphenol-diethoxylate concentrations in base-flow samples, November 4-7, 2002, and July 14-18, 2003.—Continued

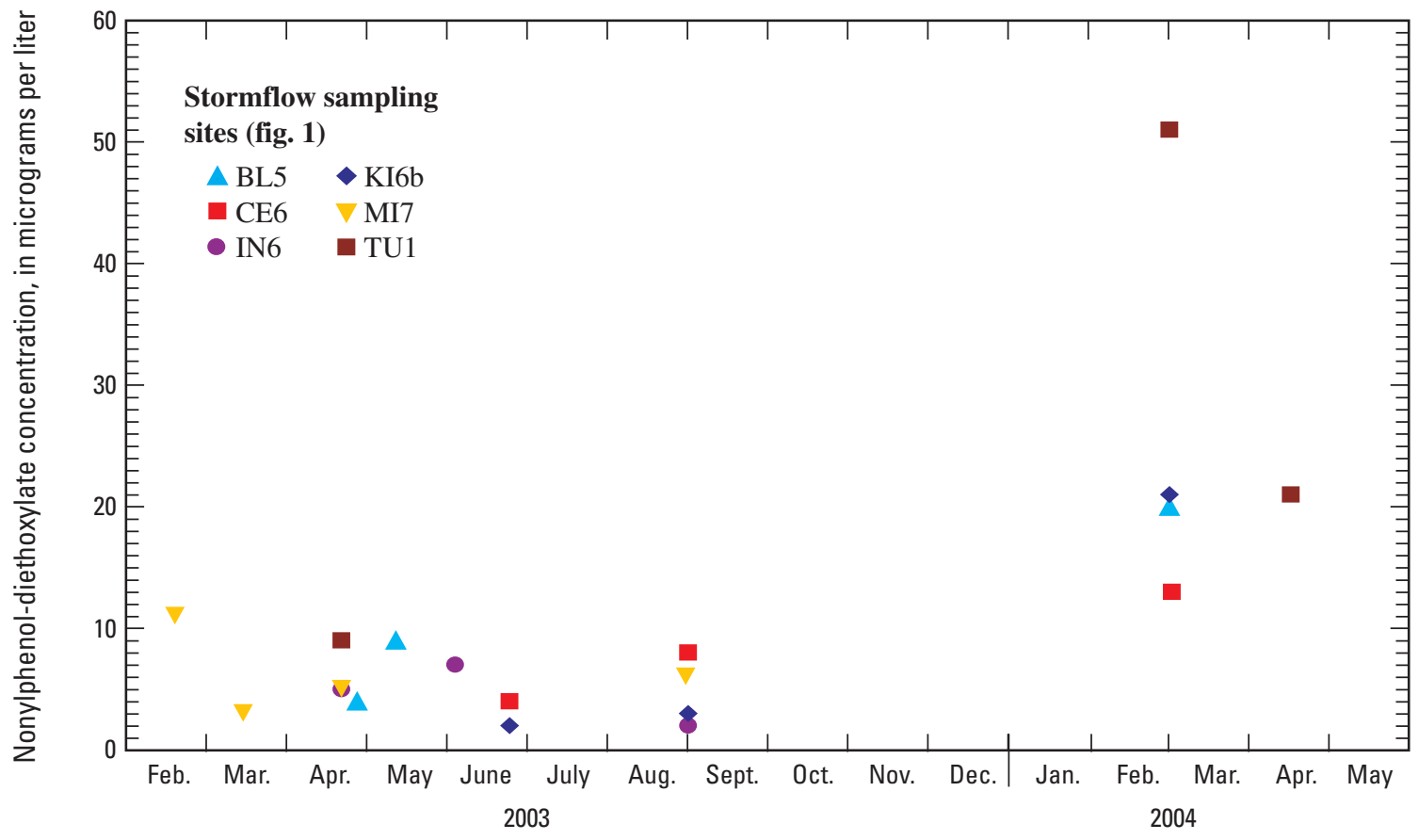

Figure 27. Nonylphenol-diethoxylate concentrations in stormflow samples, February 2003-April 2004. 

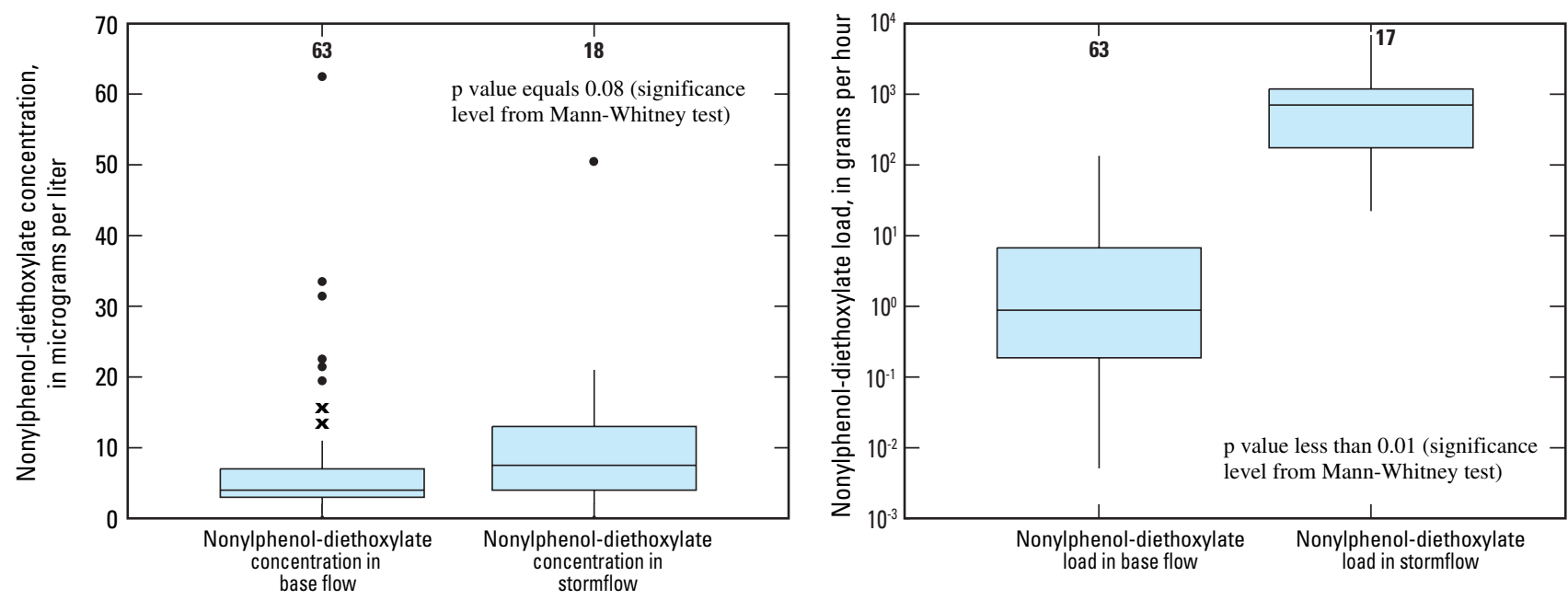

\section{EXPLANATION}

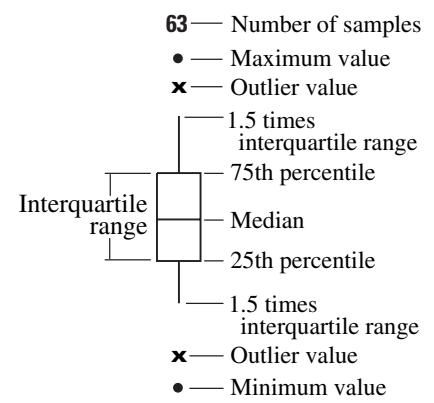

Figure 28. Nonylphenol-diethoxylate concentrations and estimated loads in base- and stormflow samples, November 2002April 2004.

systems, sanitary sewer overflows, leaking sewer lines, or illicit discharges. Industrial applications comprise 55 percent of the APEO market, remaining uses include institutional cleaning products (30 percent) and household cleaning products (Ying and others, 2002). All 4-NP concentrations in stormflow samples were less than the laboratory reporting level $(5 \mu \mathrm{g} / \mathrm{L})$ and, therefore, less than USEPA draft chronic toxicity criterion (5.9 $\mu \mathrm{g} / \mathrm{L})$.

\section{Flame Retardants and Plasticizers}

Tris(2-butoxyethyl) phosphate (TBEP), tris(2-chloroethyl) phosphate, and tris(dichlorisopropyl) phosphate are flame retardants and plasticizers that were detected at concentrations larger than laboratory reporting levels. TBEP was detected at the largest concentrations and was the only compound in this category detected at concentrations larger than $1 \mu \mathrm{g} / \mathrm{L}$. TBEP is used primarily as an ingredient in floor polishes and as a plasticizer in rubber and plastics. TBEP is expected to adsorb to sediment $\left(\mathrm{K}_{\mathrm{ow}}=4.8\right)$ and degrade to metabolites quickly after input into surface water (World Health Organization, 2000).

The largest TBEP concentrations were found in base-flow samples collected from WWTF discharges. Three of the four largest TBEP concentrations in samples from WWTF discharges were collected at trickling-filter WWTF sites MI2 (5.3 $\mu \mathrm{g} / \mathrm{L})$, TO3 (15 $\mu \mathrm{g} / \mathrm{L})$, and TU3 (27 $\mu \mathrm{g} / \mathrm{L})$. Samples from site MI1 $(7.4 \mu \mathrm{g} / \mathrm{L})$ contained the largest TBEP concentration collected upstream from WWTF discharges during the November synoptic survey. Sources of this concentration are unknown; TBEP was not detected in the base-flow sample collected from site MI1 during the July 2003 synoptic survey. Five Johnson County samples contained concentrations that exceeded maximum TBEP levels found by Kolpin and others (2002), and seven samples exceeded concentrations found by Lee and others (2004) (table 20). Five of the seven concentrations were in samples collected at or downstream from trickling-filter WWTFs (sites TO3 and TU3). Large TBEP concentrations in samples collected from these sites may be related to larger concentrations in WWTF inflows and (or) to poor removal using trickling-filter secondary treatment processes. TBEP was the only flame retardant or plasticizer in stormflow samples detected at concentrations larger than the laboratory reporting levels $(0.5 \mu \mathrm{g} / \mathrm{L})$. TBEP was detected at concentrations larger than laboratory reporting levels at site TU1 (2.7 $\mu \mathrm{g} / \mathrm{L}$, April 20, 2004, and $0.8 \mu \mathrm{g} / \mathrm{L}$, March 4, 2004), site CE6 (0.8 $\mu \mathrm{g} / \mathrm{L}$, February 14, 2003), site IN6 (0.7 $\mu \mathrm{g} / \mathrm{L}$, June 2, 2003), and site MI7 (0.6 $\mathrm{g} / \mathrm{L}$, August 30, 2003). With the exception of Cedar Creek, these sites are downstream from 
predominantly urban or urbanizing watersheds, which are more likely to contain leaking sewer lines and (or) easily transportable spillage of floor polishes, flame retardants, or plasticizers, the primary sources of TBEP.

\section{Fragrances}

Acetyl-hexamethyl-tetrahydro-napthalene (AHTN) was the only fragrance compound detected at concentrations larger than laboratory reporting levels. AHTN is a polycyclic synthetic musk used in a variety of personal care products including detergents, perfumes, and shampoos; thus, WWTF discharges are expected to be the primary sources of AHTN. AHTN has been found to persist after wastewater treatment and has a large $\mathrm{K}_{\mathrm{ow}}$ value (5.7), making it likely to accumulate in both streambed sediment and fish tissue (Heberer and others, 2001). Although the aquatic effects of AHTN are generally unknown, AHTN has been found to cause estrogenic activity in human breast cancer cells (Bitsch and others, 2002).

AHTN concentrations were largest in base-flow samples from WWTF sites; the two largest AHTN concentrations were in samples from trickling-filter facilities (site MI2, $2.3 \mu \mathrm{g} / \mathrm{L}$, July 16, 2003, and site TU3, $2.2 \mu \mathrm{g} / \mathrm{L}$, July 16, 2003). AHTN concentrations decreased rapidly in samples downstream from WWTFs, likely because of adsorption to streambed sediment and (or) biodegradation. AHTN was not studied by Kolpin and others (2002), and maximum Johnson County concentrations were smaller than those found by Lee and others (2004) (table 20). AHTN was detected only in samples from two sites (DY1, IN1) upstream from WWTF discharges; concentrations in all samples were estimated at $0.1 \mu \mathrm{g} / \mathrm{L}$, less than the laboratory reporting level of $0.5 \mu \mathrm{g} / \mathrm{L}$. AHTN was detected at less than the laboratory reporting level in 8 of 20 stormflow samples in samples from sites CE6, IN6, MI7, and TU3.

\section{Caffeine}

Caffeine was detected in 78 percent of Johnson County base-flow samples. Caffeine is a popular stimulant and is present in coffee, soda, pharmaceuticals, and a variety of other commercially available products. Caffeine is very mobile and is expected to stay in solution and migrate through soils (Seiler and others, 1999). Caffeine has been shown to be an effective marker of human-related effects in surface water (Buerge and others, 2003).

Caffeine concentrations were largest in samples from trickling-filter WWTF sites TO3 $(12 \mu \mathrm{g} / \mathrm{L})$ and TU3 $(10 \mu \mathrm{g} / \mathrm{L})$. Maximum caffeine concentrations in samples from sites IN1, TO3, and TU3 exceeded maximum concentrations in studies by Kolpin and others (2002) and Lee and others (2004) (table 20). Although influent concentrations are not known, treatment processes are considered to be important in affecting caffeine removal (Buerge and others, 2003). Comparing mean concentrations in samples from WWTFs with similar capacities (10 to $15 \mathrm{Mgal} / \mathrm{d}$ ), samples from trickling-filter discharge (sites TO3 and TU3) had much larger mean caffeine concentrations $(9.1 \mu \mathrm{g} / \mathrm{L})$ than samples from activated-sludge discharge (site IN3, design flow $9.0 \mathrm{Mgal} / \mathrm{d}$, less than $0.5 \mu \mathrm{g} / \mathrm{L}$ ).

Base-flow samples collected during the November 2002 synoptic survey from sites upstream from WWTF discharges in urban areas, sites BR1 $(0.6 \mu \mathrm{g} / \mathrm{L})$, DY1 $(0.9 \mu \mathrm{g} / \mathrm{L})$, IN1 $(6.9 \mu \mathrm{g} / \mathrm{L})$, IN2 $(1.8 \mu \mathrm{g} / \mathrm{L})$, MI1 $(0.6 \mu \mathrm{g} / \mathrm{L})$, and TU1 $(0.7 \mu \mathrm{g} / \mathrm{L})$, also had caffeine concentrations larger than the laboratory reporting level. Concentrations in samples upstream from WWTF discharges during the November 2002 synoptic survey were likely due to nonpoint sources of caffeine such as leaking sewage lines or spilled caffeinated beverages. For example, an average cup ( $8 \mathrm{oz}$ ) of coffee contains $250 \mathrm{mg}$ of caffeine, so at a hypothetical site with an average streamflow of $1 \mathrm{ft}^{3} / \mathrm{s}, 1$ cup of coffee, perfectly poured and distributed throughout the stream over 1 hour, would result in a consistent concentration of $2.5 \mu \mathrm{g} / \mathrm{L}$ of caffeine. Thus, very small inputs of caffeine from coffee, soda, or other sources can cause microgram-per-liter concentrations of caffeine in small urban streams during baseflow conditions.

Caffeine was detected in 15 of 20 stormflow samples, two of which (site TU1, $1.9 \mu \mathrm{g} / \mathrm{L}$, April 20, 2004, and site MI7, $0.7 \mu \mathrm{g} / \mathrm{L}$, February 14,2003 ) had concentrations larger than the laboratory reporting level $(0.5 \mu \mathrm{g} / \mathrm{L})$. Potential sources of caffeine during stormflow conditions include leaking sewage lines, sanitary sewer overflows, and nonpoint spillage of products containing caffeine. The widespread detections in baseand stormflow samples indicate that caffeine generally is ubiquitous in Johnson County streams, especially in more urban watersheds.

\section{Insecticides/Bird Repellents/Petroleum Compounds}

The insecticide DEET was detected at concentrations larger than the laboratory reporting level $(0.5 \mu \mathrm{g} / \mathrm{L})$ in Johnson County base-flow samples. DEET is a commonly used insecticide for control of mosquitos, flies, and ticks. There is little information on the transport and degradation of DEET in surface water, but DEET has been shown to have low acute toxicity to humans and aquatic life (U.S. Environmental Protection Agency, 1998a). Likely sources of DEET include contributions from laundry and bathing inputs to WWTFs.

During base-flow conditions, DEET was detected at concentrations larger than the laboratory reporting level only in samples collected during the July 2003 synoptic survey and then only in samples collected at or immediately downstream from trickling-filter WWTFs (site MI2, $1.4 \mu \mathrm{g} / \mathrm{L}$; site TO3, $3.0 \mu \mathrm{g} / \mathrm{L}$; and site TU3, $3.7 \mu \mathrm{g} / \mathrm{L}$ ). These concentrations were larger than maximum values found by Kolpin and others (2002) but were much less than maximum values found by Lee and others (2004) (table 20). The occurrence of DEET primarily during the July 2003 synoptic indicates that increased summer use of DEET corresponds to increased concentrations in Johnson County streams. Secondary treatment processes again appear to be a dominant factor in the occurrence of DEET in 
stream water; samples of discharge from activated-sludge treatment facilities did not contain a detection of DEET larger than the laboratory reporting level during either base-flow synoptic survey.

DEET was detected in 15 of 20 stormflow samples; however, only 1 sample (site IN6, $0.6 \mu \mathrm{g} / \mathrm{L}$, June 2, 2003) had a detection larger than the laboratory reporting level $(0.5 \mu \mathrm{g} / \mathrm{L})$. The large number of detections indicate that DEET sources are widespread throughout Johnson County, at both urban and nonurban sites; however, the samples from the most urban sites (sites IN6, MI7, and TU1) had detections of DEET in every stormflow sample.

9,10-Anthraquinone is a bird repellent and also is used in manufactured dyes and textiles. 9,10-Anthraquinone was detected only at concentrations larger than laboratory reporting levels in selected urban stormflow samples (site IN6, $0.9 \mu \mathrm{g} / \mathrm{L}$, June 2, 2003, and site TU1, $0.9 \mu \mathrm{g} / \mathrm{L}$, April 19, 2003, $0.8 \mu \mathrm{g} / \mathrm{L}$, March 4, 2004, $3.2 \mu \mathrm{g} / \mathrm{L}$, April 20, 2004). Detections of this compound in stormflow samples from urban watersheds suggest that sources of this compound are nonpoint, possibly from application of the compound as a geese repellent or by degradation of anthracene (component of coal tar, combustion byproduct) (U.S. Environmental Protection Agency, 2005b).

Carbazole is an product in coal-tar creosote found in crude oil and is a potential mutagen (Benedik and others, 1998). Carbazole was only detected at a concentration larger than laboratory reporting levels in one stormflow sample (site TU1, $1.8 \mu \mathrm{g} / \mathrm{L}$, April 20, 2004) likely due to street runoff containing coal tar (from street paving and roof usage) and petroleum byproducts (oil and gasoline). Carbazole had six total detections in stormflow samples, all at sites with the most impervious surface area (sites IN6, MI7, and TU1, table 5).

\section{Sterols and Stanols}

The sterols and stanols 3-beta-coprostanol (coprostanol), beta-sitosterol (sitosterol), beta-stigmastanol (stigmastanol), and cholesterol were detected at concentrations larger than laboratory reporting levels in Johnson County streams. Coprostanol is produced by microbial reduction of cholesterol in some mammals and humans and has been found to be a reliable fecal indicator (Leeming and others, 1996; Chan and others, 1998; Isobe and others, 2002). Sitosterol and stigmastanol generally are found in the epicuticular waxes of vascular plants as well as in pulp from papermills (Weete, 1976; Cook and others, 1997). Cholesterol is associated primarily with animal fecal material (Lee and others, 2004). All coprostanol and cholesterol detections larger than laboratory reporting levels in base-flow samples were collected at sites at, and downstream from, trickling-filter WWTFs (site MI2, 2.0 and $3.0 \mu \mathrm{g} / \mathrm{L}$, respectively; site TO3, 5.0 and $10 \mu \mathrm{g} / \mathrm{L}$, respectively; and site TU3, 5.0 and $8.0 \mu \mathrm{g} / \mathrm{L}$, respectively), indicating that WWTF treatment processes may affect the occurrence of these compounds. Sitosterol and stigmastanol did not have an obvious detection pattern and were detected in the largest concentrations in base- flow samples from site MI7 (3.0 and $4.0 \mu \mathrm{g} / \mathrm{L}$, respectively) and site TO3 (3.0 and $3.0 \mu \mathrm{g} / \mathrm{L}$, respectively).

Sterols and stanols were not detected at concentrations larger than laboratory reporting levels in stormflow samples, with the exception of one cholesterol value at site MI7 $(3.0 \mu \mathrm{g} / \mathrm{L}$, August 30, 2003). Coprostanol was estimated at concentrations less than laboratory reporting levels in 2 of 20 samples, sitosterol in 4 of 20 samples, stigmastanol in 5 of 20 samples, and cholesterol in 7 of 20 samples. Samples with detections less than laboratory reporting levels did not appear to be more or less frequent at any one of the stormflow sites.

\section{Pharmaceutical Compounds}

Ten sites were sampled for 22 pharmaceutical compounds (table 6) exclusively during base-flow synoptic surveys; no stormflow samples were analyzed for pharmaceuticals. Caffeine data were excluded from this analysis because of duplicate analysis in wastewater compound methodologies. Sites BL6, BL7, BR2, CE1 (only during July 2003 synoptic), IN2, IN3, IN4, MI7, TU2, and TU3 were sampled for pharmaceutical compounds. The number of pharmaceutical detections and total pharmaceutical concentrations were largest in WWTF discharge samples and in samples from sites downstream from WWTF discharges (sites BL7, IN4, MI7) (fig. 29). Samples from a trickling-filter WWTF (site TU3) had the largest total concentrations of pharmaceutical compounds.

Cotinine was the only pharmaceutical compound detected in samples from every site. Cotinine is formed when the human body metabolizes nicotine, a component of cigarette smoke. The largest cotinine concentrations were found in samples from site TU3 (0.74 $\mu \mathrm{gL}$, November 7, 2002; $0.63 \mu \mathrm{g} / \mathrm{L}$, July 16, 2003); samples from all other sites had cotinine concentrations less than $0.1 \mu \mathrm{g} / \mathrm{L}$. The largest concentrations of many pharmaceutical compounds were found in samples from site TU3, including acetaminophen $(2.9 \mu \mathrm{g} / \mathrm{L}$, November 7, 2002; $2.6 \mu \mathrm{g} / \mathrm{L}$, July 16, 2003) and 1,7-dimethylxanthine (caffeine metabolite) $(4.3 \mu \mathrm{g} / \mathrm{L}$, November 7, 2002; $5.1 \mu \mathrm{g} / \mathrm{L}$, July 16 , 2003). All other pharmaceuticals were detected at less than microgram-per-liter levels and generally had the largest concentrations in samples from WWTF sites BL6, IN3, or TU3.

\section{Streambed-Sediment Quality}

Streambed-sediment samples (15 total) were collected from 15 sites in nine watersheds (sites BI1, BL3, BL5, CA1, CE1, CE6, IN3a, IN6, KI5, KI6b, MI1, MI4, MI7, TO2, and TU1) to determine contaminant source areas, to evaluate contaminant fate and transport, and to estimate the toxicity of streambed sediment. Eight of the 15 sites were located downstream from municipal WWTF discharges. Sediment was sieved to less than $63 \mu \mathrm{m}$ to eliminate sediment-size effects on chemical concentrations. A variety of constituents, such as trace elements, bacteria, and organic compounds, often occur in 
A. Number of detections

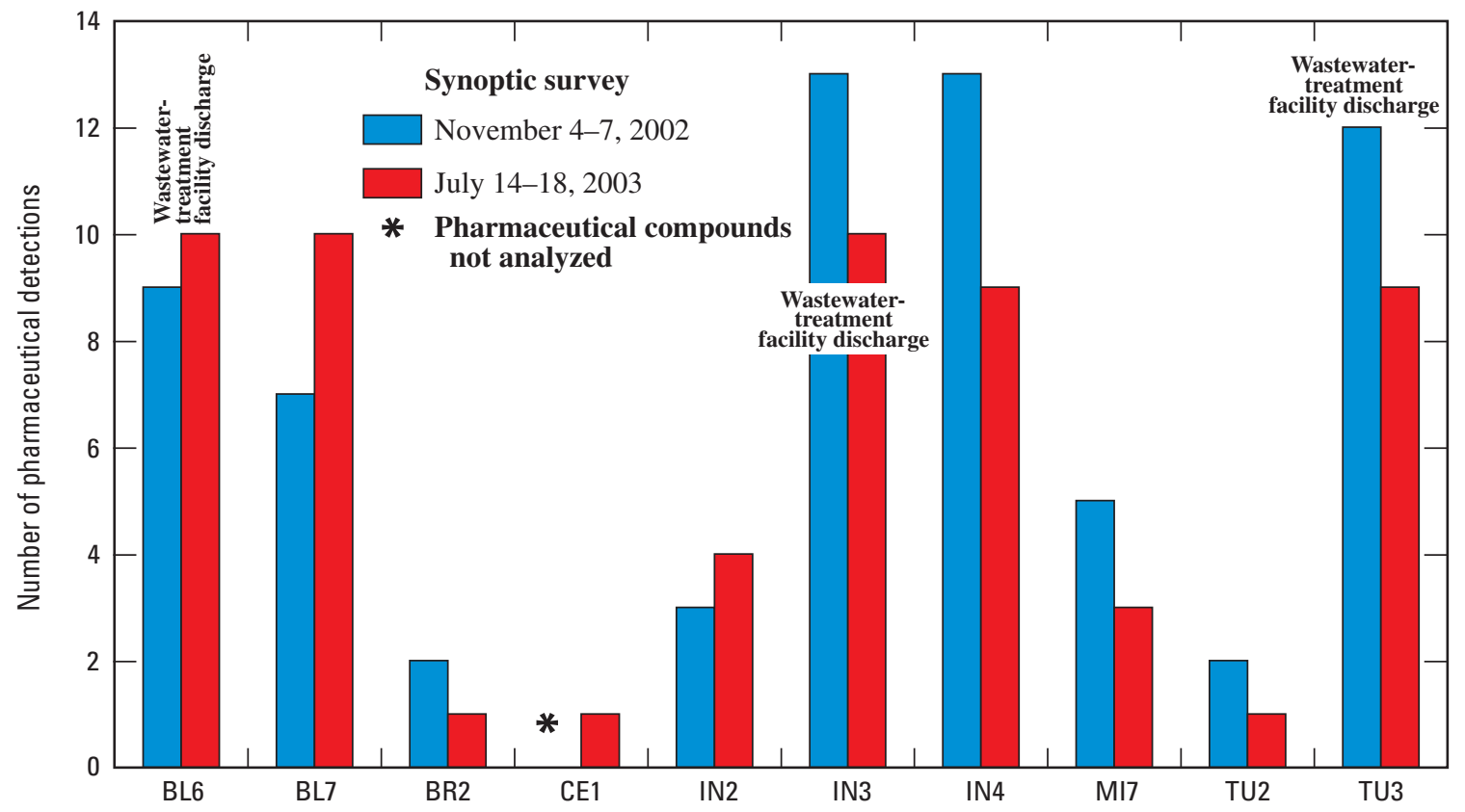

$B$. Total concentrations

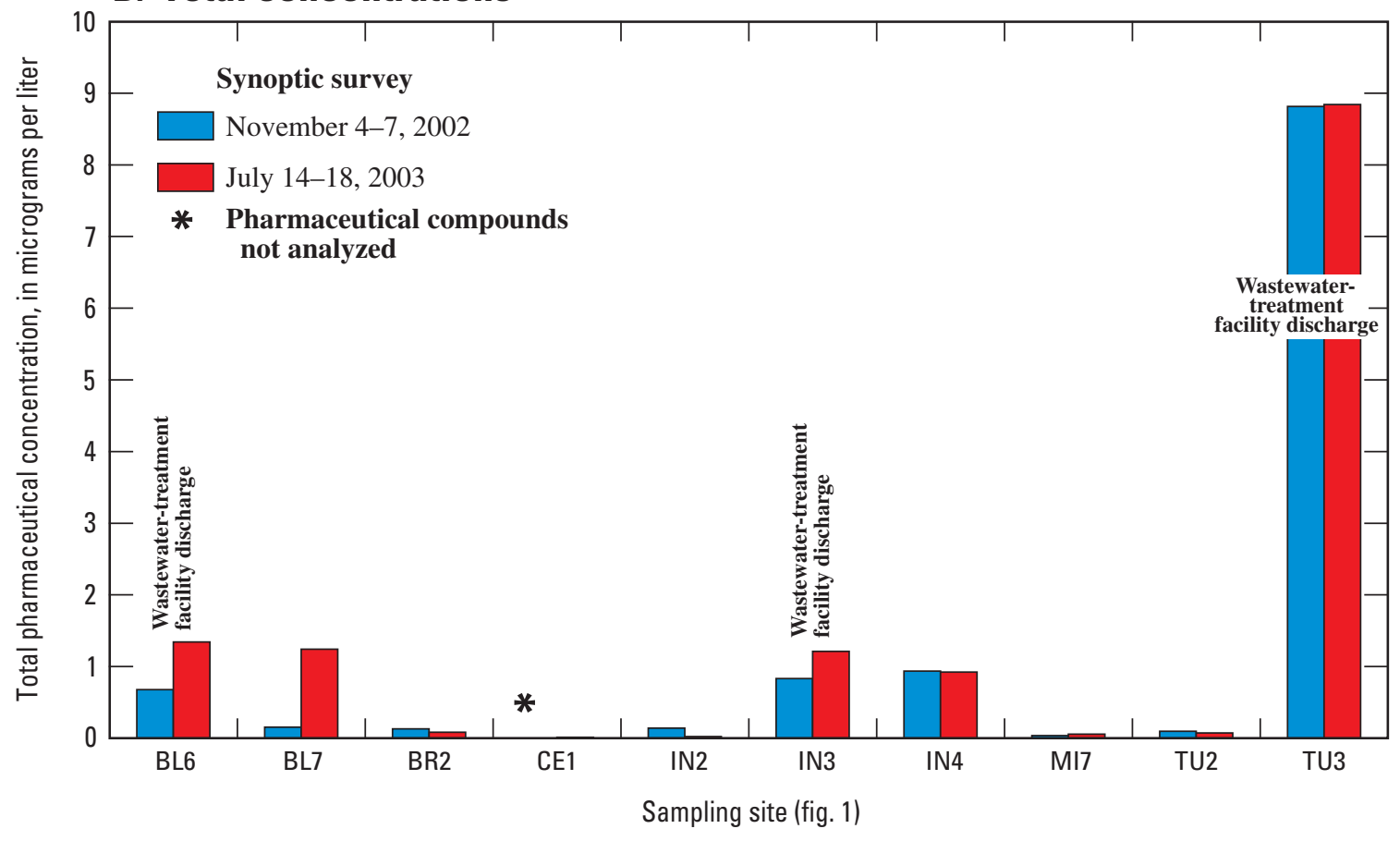

Figure 29. ( $A$ ) Number of pharmaceutical compound detections and $(B)$ total concentrations in selected baseflow samples, November 4-7, 2002, and July 14-18, 2003. 
larger concentrations in sediment than in the overlying water column (Van Donsel and Geldreich, 1971; Horowitz, 1991). Contaminated sediment can be toxic to benthic organisms and can bioaccumulate in fish and mammals (U.S. Environmental Protection Agency, 2000a).

USEPA (1998b) has established two sets of sedimentquality guidelines for selected trace elements and organic compounds - the threshold effects level (the TEL) and the probable effects level (PEL). These guidelines are used for comparison with constituents analyzed from Johnson County streambed sediment. The smaller of the two guidelines (TEL) is used to represent the concentrations below which toxic effects rarely occur. In the range between the TEL and PEL, toxic effects occasionally occur, and above the PEL guideline toxic effects frequently occur. The guidelines are used by USEPA as screening tools and do not have regulatory implications. This cautionary statement is made because, although biological-effects correlation to trace element concentrations identifies levels-ofconcern concentrations associated with the likelihood of adverse organism response, the particular procedure used may not demonstrate that a particular chemical is solely responsible. Biological-effects correlations may not indicate direct causeand-effect relations because sediment samples may contain a mixture of chemicals that contribute to adverse effects in varying degrees. Therefore, for any given site, these guidelines may be over- or underprotective (U.S. Environmental Protection Agency, 1998b).

Sediment-quality analysis is divided into six sectionstotal organic carbon and nutrients, trace elements, bacteria, pesticides (including certain pesticides and pesticide degradation products), wastewater compounds, and pharmaceutical compounds. Complete results of analysis of all streambed-sediment quality samples are available on the USGS Web site (http://ks.water.usgs.gov/Kansas/studies/qw/joco) and are on file with USGS in Lawrence, Kansas.

\section{Total Organic Carbon and Nutrients}

Large concentrations of organic carbon in streambed sediment are indicative of organic enrichment and can have a substantial effect on the chemical makeup of sediment. Organic carbon forms complexes with metal species and improves adsorption of many contaminants (Horowitz, 1991). Total organic carbon concentrations ranged from $8,000 \mathrm{mg} / \mathrm{kg}$ in a sample from site TU1 to $61,000 \mathrm{mg} / \mathrm{kg}$ in a sample from site BI1.

Major sources of nutrients in freshwater sediment include fertilizers, livestock production, WWTFs, and septic systems. There are currently (2005) no published guidelines for nutrients in freshwater sediment. Total nitrogen concentrations in Johnson County streambed-sediment samples ranged from $1,000 \mathrm{mg} / \mathrm{kg}$ (sites BL3 and TU1) to $6,800 \mathrm{mg} / \mathrm{kg}$ (site BI1) (fig. 30). Total phosphorus concentrations ranged from $620 \mathrm{mg} / \mathrm{kg}$ ( site CA1) to $2,600 \mathrm{mg} / \mathrm{kg}$ (site BI1). WWTF discharges were a likely source of the large nutrient concentrations; samples from sites with the two largest concentrations (sites BI1 and IN3a) were both collected downstream from WWTF discharges.

\section{Trace Elements}

Trace elements are important constituents of concern in sediment because of their potential toxicity to living organisms. Trace elements analyzed in streambed sediment are listed in table 6. Many trace elements originate naturally in rock formations. However, automobile emissions, human and animal waste, fertilizers, liming materials, pesticides, as well as residential and industrial uses comprise potential sources of trace elements in streambed sediment.

Many streambed-sediment samples had trace element concentrations that exceeded TELs for several of the nine trace elements with sediment-quality guidelines; however, of these nine trace elements, only two analytes exceeded PEL guidelines (fig. 31). Samples from sites BI1 (downstream from WWTF, nonurban use), CE1 (downstream from limestone quarry), and IN6 (downstream from WWTF, urban use) had trace element concentrations larger than the TEL criteria in seven of nine samples, whereas samples from urban sites upstream from WWTF discharges (sites MI1, TO2, and TU1) had concentrations larger than the TEL in six of nine samples. The PEL for nickel $(42.8 \mu \mathrm{g} / \mathrm{kg})$ was exceeded in a sample from site CE1 $(43 \mu \mathrm{g} / \mathrm{kg})$. Nickel concentrations in Lake Olathe bottom sediment were $39 \mu \mathrm{g} / \mathrm{kg}$ (Mau, 2000). Limestone quarries upstream from sites CE1, MI1, TO2, and TU1 could be the source of elevated nickel concentrations in streambed sediment; however average nickel concentrations in eastern Kansas limestones have been found to be $10 \mathrm{mg} / \mathrm{kg}$ (Runnels and Schleicher, 1956). The PEL for silver $(1.77 \mu \mathrm{g} / \mathrm{kg})$ was exceeded in a sample from site IN3a $(2.3 \mu \mathrm{g} / \mathrm{kg})$. Sources of silver found in wastewater discharge most likely are from photographic contributions (Shakibaie and others, 1999).

\section{Bacteria}

Fecal coliform, Escherichia coli (E. coli), and enterococci are indicator bacteria typically used to represent the pathogenicity of surface water. There are no USEPA guidelines or typical background densities published for indicator bacteria in freshwater streambed sediment. Indicator bacteria densities have been found to be 100 to 1,000 times larger at the mud/surfacewater interface than in the overlying water column (Van Donsel and Geldreich, 1971). Fecal coliform bacteria have been observed to reproduce in streambed sediment and have shown improved survival rates in smaller particle-size sediment. In addition, fecal coliform bacteria have shown higher mortality rates in sediment than fecal streptococci bacteria, a bacteria class similar in structure to that of enterococci (Howell and others, 1996).

The largest fecal coliform and E. coli densities in Johnson County streambed sediment were in a sample from site IN3a, the site located closest to a WWTF discharge (fig. 32). Samples 

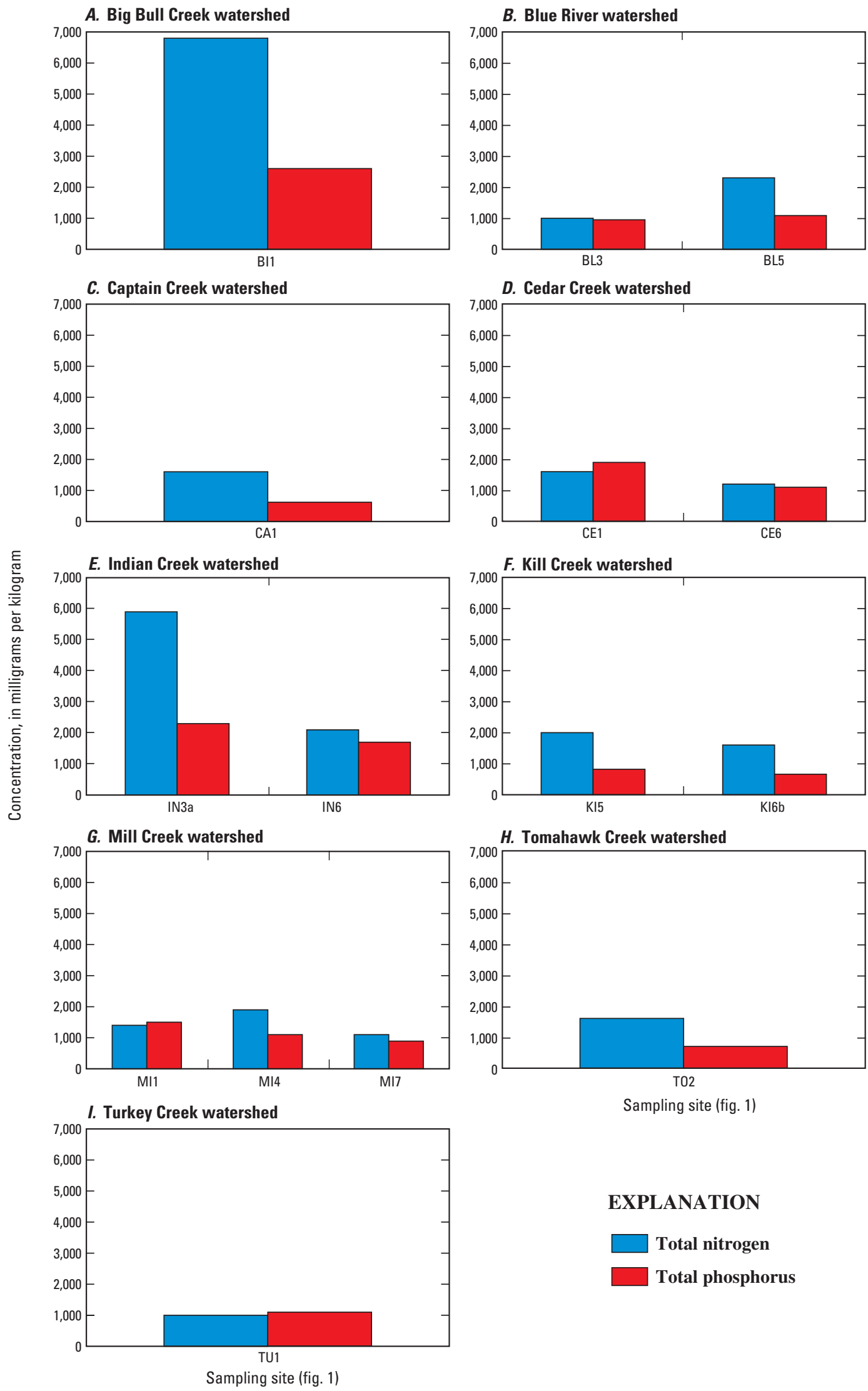

Sampling site (fig. 1)

EXPLANATION

Total nitrogen

Total phosphorus

Figure 30. Total nitrogen and total phosphorus concentrations in streambed-sediment samples, March 31-April 3, 2003. 


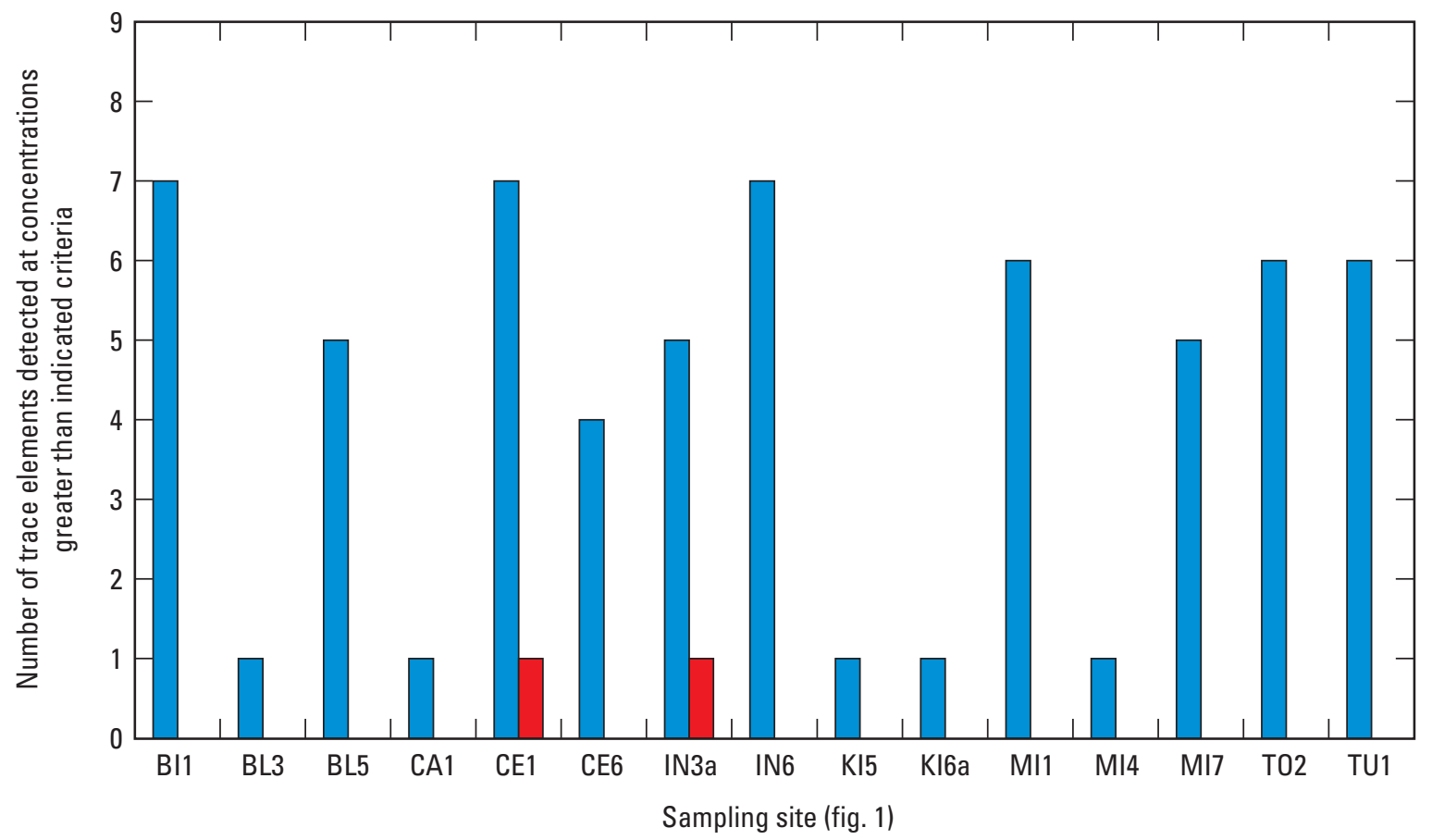

EXPLANATION

Number of trace elements exceeding U.S.

Environmental Protection Agency threshold effects level guidelines (1998b)

Number of trace elements exceeding U.S.

Environmental Protection Agency probable effects level guidelines (1998b)

Figure 31. Number of trace elements detected at concentrations greater than U.S. Environmental Protection Agency (1998b) sediment-quality threshold effects level (TEL) and probable effects level (PEL) in Johnson County streambed-sediment samples, March 31-April 3, 2003.

from urban and nonurban sites exhibited different enterococci/ fecal coliform ratios. Samples from urban sites (IN3a, IN6, MI1, MI4, MI7, TO2, TU1) had a mean enterococci/fecal coliform ratio of 3.8, whereas samples from more nonurban sites (BI1, BL3, BL5, CA1, CE1, CE6, KI5, KI6b) had a mean enterococci/fecal coliform ratio of 17.3. Whether this phenomenon is related to different sources of fecal contamination, bacteria survival characteristics, or other factors is unknown.

The occurrence of indicator bacteria in streambed sediment increases the possibility that resuspension of this material may be a substantial source of large bacteria densities determined in samples (fig. 20) at stormflow sampling sites (BL5, CE6, IN6, KI6b, MI7, TU1; fig. 1). To investigate this possibility, densities of $E$. coli determined in streambed sediment at the six stormflow sampling sites were converted to theoretical stormflow densities on the basis of suspended-sediment concentrations determined in stormflow samples. This theoretical conversion was performed by first dividing the streambedsediment densities of $E$. coli (in colonies per gram dry weight) by $1,000 \mathrm{mg} / \mathrm{g}$, multiplying by the suspended-sediment concentration (in milligrams per liter) in each stormflow sample, and then multiplying by 0.1 to achieve colonies per $100 \mathrm{~mL}$. The results were theoretical E. coli densities whose source was completely based on resuspension of streambed material. These theoretical densities were compared to the analytically determined E. coli densities in the stormflow samples. On the basis of these comparisons for the six stormflow sampling sites, it was determined that, on average, resuspension of streambed sediment would account for less than 1 percent of the $E$. coli densities analytically determined in stormflow samples. This indicates that resuspension of streambed sediment is likely not the dominant source of E. coli (and potentially other indicator bacteria) during stormflow conditions in Johnson County watersheds. Nonpoint-source runoff and WWTF bypasses are more likely sources of indicator bacteria densities during stormflow conditions. 

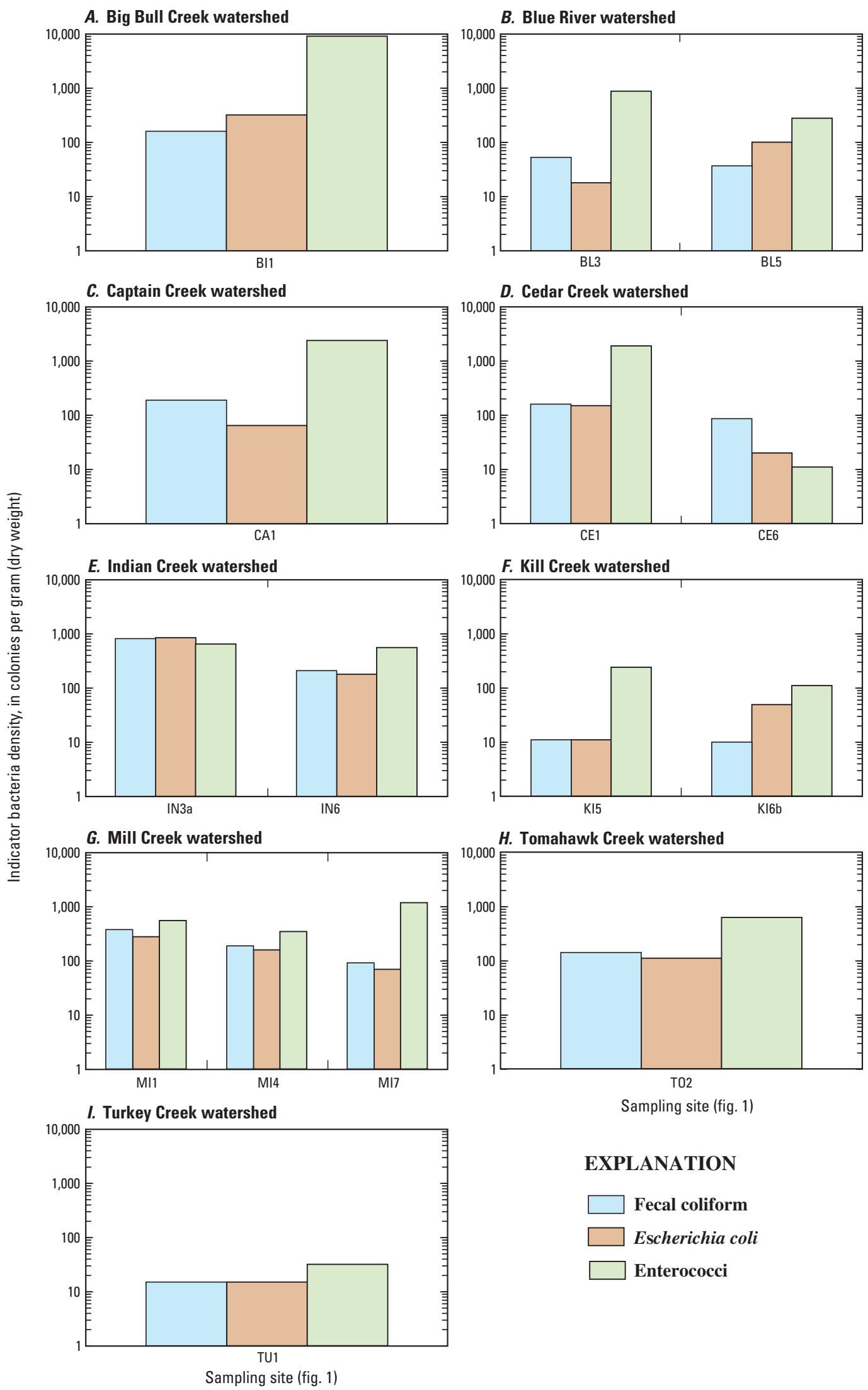

Sampling site (fig. 1)

EXPLANATION

Fecal coliform

Escherichia coli

Enterococci

Figure 32. Indicator bacteria densities in streambed-sediment samples, March 31-April 3, 2003. 


\section{Pesticides}

Thirty-eight pesticide compounds and metabolites (table 6) were analyzed in Johnson County streambed-sediment samples. Pesticide compounds that were analyzed in streambed-sediment samples are generally hydrophobic and are very persistent in the environment. Because of this, they have long environmental lifetimes and accumulate in the fatty tissue of organisms (Masters, 1991).

Samples from 7 of 15 sampling sites did not have detections of pesticide compounds. Eleven compounds analyzed were detected in streambed-sediment samples. Of the compounds that were detected, four are components of total chlordane, and three are components of total dichloro-diphenyltrichloroethane (DDT). Heptachlor, heptachlor epoxide, and total PCBs also were detected, but concentrations were all estimated at less than laboratory reporting levels. Dieldrin was the only compound other than chlordane or DDT to have concentrations larger than laboratory reporting levels. The most detections and largest concentrations of organochlorine compounds were found in samples from urban sites, primarily in the Indian and Mill Creek watersheds.

The compounds cis-chlordane, trans-chlordane, cisnonachlor, and trans-nonachlor were all detected; together these compounds compose total chlordane. Chlordane is an insecticide that has both agricultural and residential uses and was banned from use by USEPA in 1988 (U.S. Environmental Protection Agency, 2004a). Watersheds with more agricultural land uses (Blue River and Kill Creek) have TMDLs for chlordane, but chlordane was not detected in samples from either of these watersheds (fig. 33). All total chlordane detections were in samples from more urban sites (IN3a, IN6, MI1, MI4, TU1) and were larger than the $4.79 \mu \mathrm{g} / \mathrm{kg}$ USEPA PEL. The more urban watersheds of Indian and Mill Creeks do not have TMDLs for chlordane (related to fish-tissue consumption) but recorded the largest chlordane concentrations in streambedsediment samples. The sample from site MI1 had the largest concentration of chlordane $(26 \mu \mathrm{g} / \mathrm{kg})$.

Total DDT comprises the organochlorine compounds p,p'DDD, p,p'DDE, and p,p'DDT. DDT was banned for use as a commercial pesticide in 1972 (U.S. Environmental Protection Agency, 1972). Streambed-sediment samples from sites IN6 and MI1 had the only detections of DDT compounds. The sample from site IN6 had detections of two metabolitesp,p'DDE $(3 \mu \mathrm{g} / \mathrm{kg})$, which was larger than the USEPA TEL guideline $(2.07 \mu \mathrm{g} / \mathrm{kg})$, and p,p'DDT (5 $\mu \mathrm{g} / \mathrm{kg})$, which was larger than the USEPA PEL guideline $(4.77 \mu \mathrm{g} / \mathrm{kg})$. The sample from site MI1 had detections of all three DDT metabolites; concentrations of p,p'DDD $(5 \mu \mathrm{g} / \mathrm{kg})$ and p,p'DDE $(6 \mu \mathrm{g} / \mathrm{kg})$ were larger than the USEPA TEL, whereas p,p'DDT ( $5 \mu \mathrm{g} / \mathrm{kg})$ concentrations were larger than the PEL guideline. Total DDT concentrations in samples from sites IN6 and MI1 were 8 and $16 \mu \mathrm{g} / \mathrm{kg}$, respectively, and were larger than the USEPA TEL guideline of $3.89 \mu \mathrm{g} / \mathrm{kg}$ but less than the PEL guideline of $51.7 \mu \mathrm{g} / \mathrm{kg}$.
Dieldrin is a metabolite of aldrin, both pesticides that were phased out of use between 1974 and 1987 (Agency for Toxic Substances and Disease Registry, 2002). However, dieldrin can still be found in freshwater sediment due to its chemical stability. Dieldrin is known to be toxic and have estrogenic effects on aquatic life (Soto and others, 1994). Dieldrin was detected in samples from sites CA1 (1 $\mu \mathrm{g} / \mathrm{kg})$, IN6 (6 $\mu \mathrm{g} / \mathrm{kg})$, MI $(2 \mu \mathrm{g} / \mathrm{kg})$, and MI4 (2 $\mu \mathrm{g} / \mathrm{kg})$; all concentrations were larger than the USEPA TEL of $0.715 \mu \mathrm{g} / \mathrm{kg}$. However, dieldrin laboratory reporting levels ranged from 1 to $3 \mu \mathrm{g} / \mathrm{kg}$; thus all detections were larger than USEPA TELs. The sample from site IN6 recorded the only concentration larger than the USEPA PEL of $4.3 \mu \mathrm{g} / \mathrm{kg}$. Concentrations of chlordane, DDT, and dieldrin in Johnson County streambed-sediment samples likely are due to persistence of these compounds in the streambed sediment from their use prior to each respective compound ban.

\section{Wastewater-Indicator Compounds}

Sixty organic wastewater compounds were analyzed in streambed-sediment samples (table 6); 33 of these compounds were detected. Samples from every site had detections of at least eight wastewater compounds (fig. 34). The largest concentrations of wastewater compounds were found in samples from sites near WWTF discharges due to the tendency of many organic compounds to adsorb to sediment. Of the four sites with samples that had more than 23 detections, three sites were close to WWTF discharges (sites IN3a, IN6, and MI4). However, three of the seven sites with samples that had the most detections of wastewater compounds were located upstream from WWTF discharges and downstream from urban watersheds. Samples from sites with more nonurban land uses (sites BI1, BL3, BL5, CA1, CE1, CE6, KI5, KI6b) averaged 12 wastewater compound detections; samples from more urban sites (IN3a, IN6, MI1, MI4, MI7, TO2, TU1) averaged 22 detections. Streambed-sediment samples from sites downstream from and close to WWTF discharges (sites BI1, IN3a, IN6) had the largest total concentrations of wastewater compounds.

Wastewater compounds were categorized as polycyclic aromatic hydrocarbons (PAHs), detergent metabolites and phenols, fragrances, sterols and stanols, and other compounds (consisting of antimicrobials, flame retardants, hormones, insecticides, and plasticizers). Only compounds detected at concentrations larger than laboratory reporting levels are discussed in the following sections.

\section{PAHs}

The occurrence of polycyclic aromatic hydrocarbons (PAHs) in environmental samples typically is related to human activities, such as "slash and burn" agriculture, automobile use, and fossil fuel combustion (Mitra and Bianchi, 2003). Many PAHs are known or suspected carcinogens and can be toxic to aquatic life individually or in combination with other PAHs. PAHs are hydrophobic and likely to adsorb to streambed 


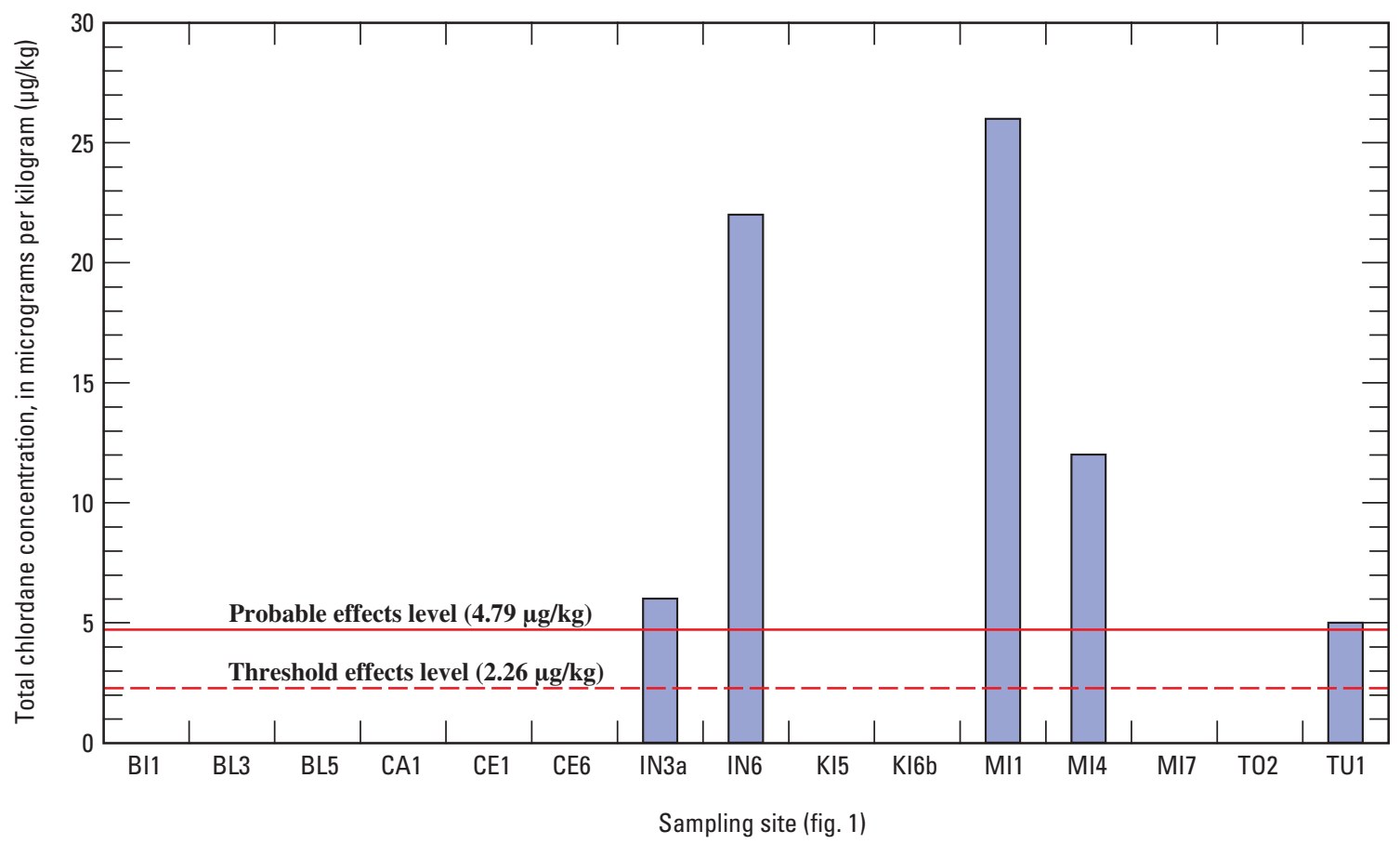

Figure 33. Total chlordane concentrations in streambed-sediment samples, March 31-April 3, 2003. Threshold and probable effects levels from U.S. Environmental Protection Agency (1998b).

sediment (Agency for Toxic Substances and Disease Registry, 1995a). Van Metre and others (2000) reported that PAH concentrations increased in lake sediment from 10 urban watersheds in the United States in the past 20 years. The larger increases in PAH concentrations may be associated with urban expansion and increasing vehicle traffic. The PAHs anthracene, benzo(a)pyrene, fluoranthrene, naphthalene, phenanthrene, and pyrene have USEPA TEL and PEL streambed-sediment guidelines.

Streambed-sediment samples from 14 of 15 sites (all except site KI5) had detections of at least one PAH; samples from sites CE1, IN3a, IN6, KI6b, MI1, MI4, MI7, TO2, and TU1 had detections of all sampled PAH compounds (table 21). Samples from urban sites IN3a, IN6, and TO2 consistently had the largest PAH concentrations; concentrations in samples from these sites exceeded USEPA PELs for fluoranthrene and phenanthrene. Additionally, samples from sites IN3a and IN6 exceeded USEPA PELs for benzo(a)pyrene and pyrene. Samples from sites in the urbanizing Mill Creek watershed (sites MI1, MI4, and MI7) and site TU1 (in the older urban part of Johnson County) consistently had detections of PAHs but at smaller concentrations than samples from the Indian and Tomahawk Creek sites (IN3a, IN6, and TO2).

\section{Detergent Metabolites and Phenols}

Detergent metabolites and phenols include 4-methyl phenol (para-cresol), bisphenol A, phenol, and metabolites of the alkylphenol ethoxylates (APEOs). Para-cresol is a common wood preservative, is readily degradable in the environment, and is a potential carcinogen (Agency for Toxic Substances and
Disease Registry, 1995b). Bisphenol A (BPA) is used predominantly as an intermediate in the production of polycarbonate plastics and epoxy resins and is known to exert estrogenic effects on some aquatic-life species (Ying and others, 2002; Fent and others, 2003). BPA is expected to adsorb to sediment in the environment (Fent and others, 2003). Phenol is a known toxin; the largest uses are in the plywood, adhesive, construction, automotive, and appliance industries (U.S. Environmental Protection Agency, 2002). APEOs are a class of surfactants used in commercial, industrial, and residential applications.

Para-cresol was detected in samples from every site; samples from 8 of 15 sites had detections larger than the laboratory reporting level $(100 \mu \mathrm{g} / \mathrm{kg})$. The largest concentrations were in samples from sites IN3a $(6,300 \mu \mathrm{g} / \mathrm{kg})$, CE1 $(2,300 \mu \mathrm{g} / \mathrm{kg})$, as well as sites MI4 and TO2 (2,000 $\mu \mathrm{g} / \mathrm{kg}$ each). BPA was detected in three samples from 15 streambedsediment sites; a sample from site $\mathrm{TO} 2$ had the only detection $(140 \mu \mathrm{g} / \mathrm{kg})$ larger than the laboratory reporting level $(100 \mu \mathrm{g} / \mathrm{kg})$. Phenol was detected in samples from 12 of 15 sites; samples from six sites had concentrations larger than the laboratory reporting level $(100 \mu \mathrm{g} / \mathrm{kg})$. Samples from sites TO2 $(510 \mu \mathrm{g} / \mathrm{kg})$ and IN3a $(480 \mu \mathrm{g} / \mathrm{kg})$ had the largest phenol concentrations.

The hydrophobic properties of APEOs suggest that they will adsorb to streambed sediment in much larger concentrations than in surface water (Ying and others, 2002). APEOs were not detected in samples from sampling sites in the Big Bull Creek, Blue River, Captain, Kill, and Turkey Creek watersheds. There were no detections of octylphenol ethoxylates, but there were detections of 4-nonylphenol (4-NP), nonylphenol- 


\section{A. Number of detections}

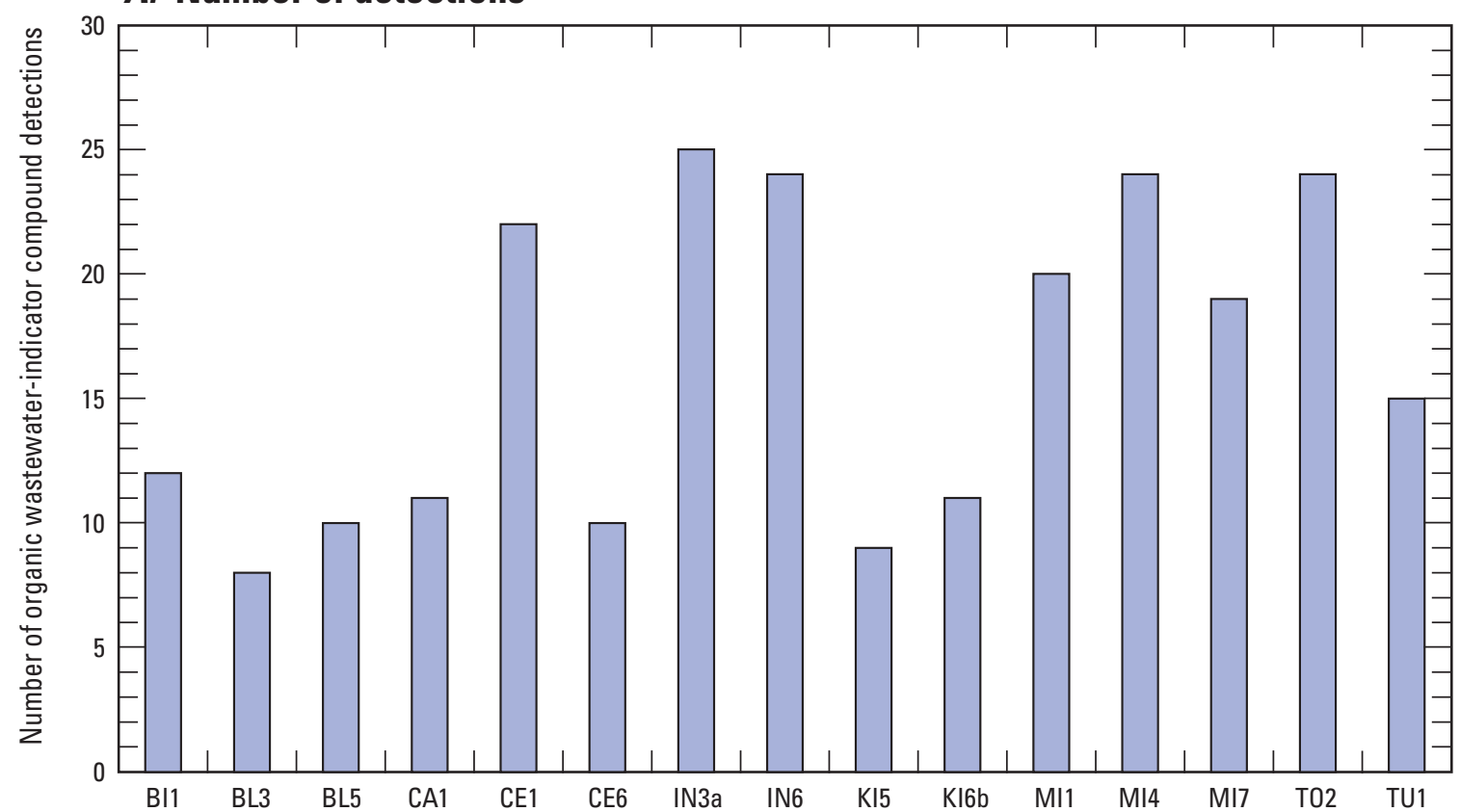

\section{B. Total concentration}

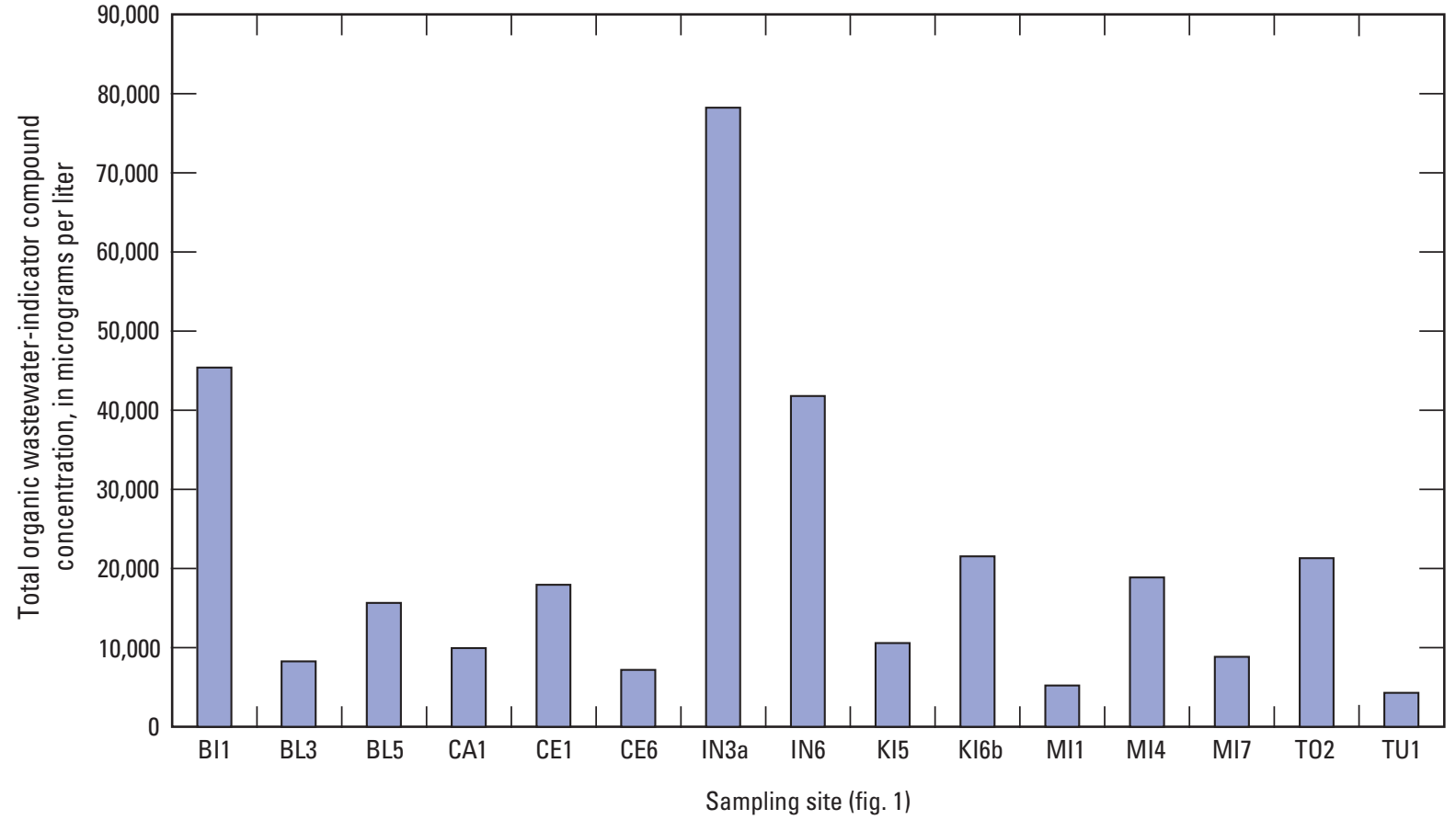

Figure 34. (A) Number of organic wastewater-indic ator compound detections and (B) total concentrations in streambed-sediment samples, March 31-April 3, 2003. 
Table 21. Concentrations of polycyclic aromatic hydrocarbons (PAHs) and metabolites in streambed-sediment samples collected from selected Johnson County streams, northeastern Kansas, March 31-April 3, 2003.

[LRL, laboratory reporting level; USEPA TEL, U.S. Environmental Protection Agency (1998b) threshold effects level; USEPA PEL, U.S. Environmental Protection Agency (1998b) probable effects level; $\mu \mathrm{g} / \mathrm{kg}$, micrograms per kilogram; <, less than; E, estimated]

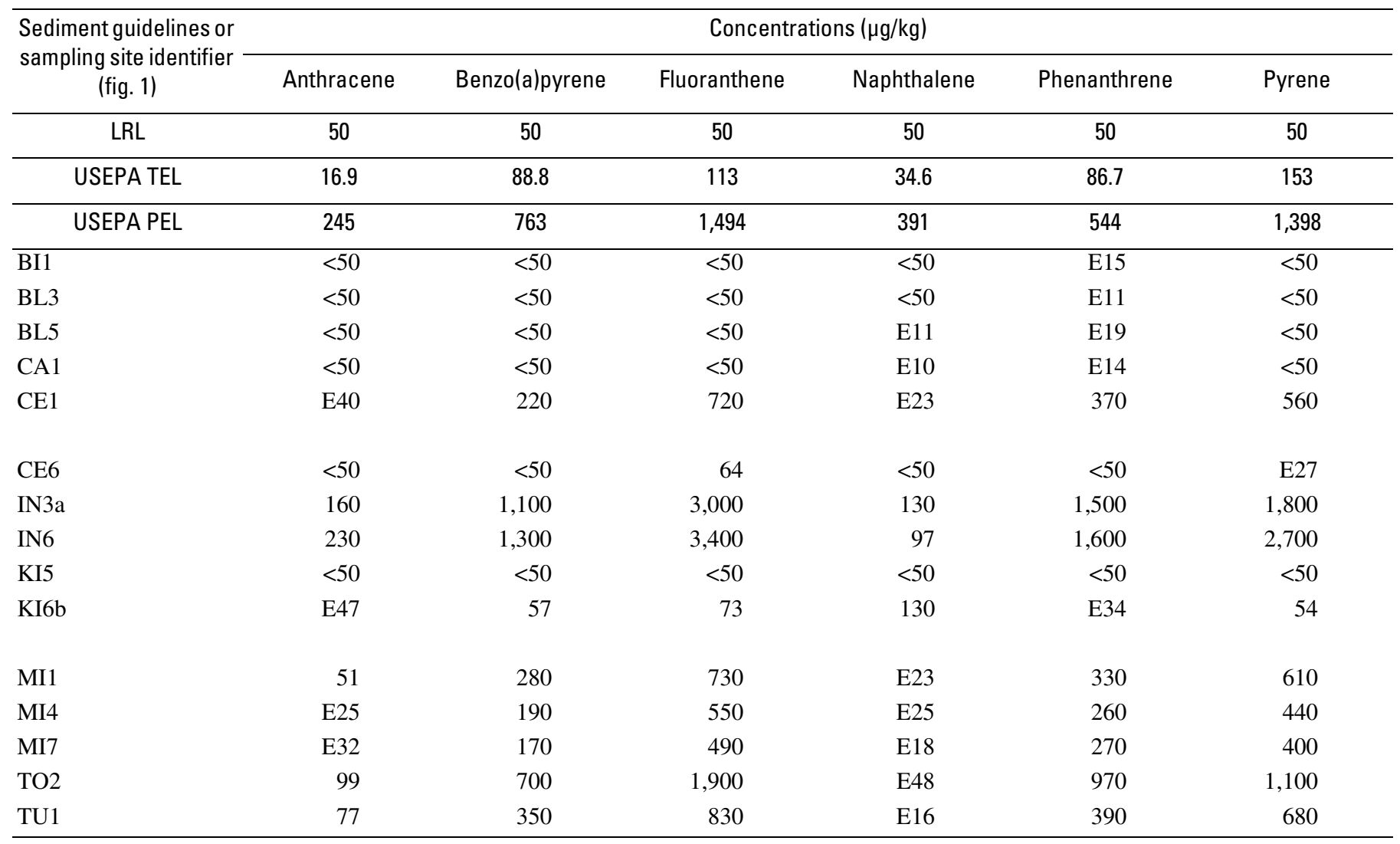

ethoxylate (NPEO1), and nonylphenol-diethoxylate (NPEO2) (fig. 35). The largest detections of nonylphenol compounds were in samples from sites located immediately downstream from WWTF discharges (sites IN3a and IN6). NPEO2 had the largest concentrations of APEO metabolites in all samples. Samples from sites CE1, MI1, MI4, MI7, and TO2 had detections of NPEO2, and samples from sites CE1, MI4, and TO2 also had detections of NPEO1. Samples from four sites had detections of 4-NP; however, only the sample from site IN3 $(950 \mu \mathrm{g} / \mathrm{kg})$ and the sample from site TO2 $(560 \mu \mathrm{g} / \mathrm{kg})$ had 4-NP concentrations larger than the laboratory reporting level (500 $\mu \mathrm{g} / \mathrm{kg})$.

Concentrations of nonylphenol compounds in Johnson County streambed sediment were within ranges found in other streambed-sediment studies (Ferguson and others, 2001; Isobe and others, 2002). Given that $1 \mathrm{~L}$ of water weighs $1 \mathrm{~kg}$, concentrations of all nonyphenol compounds in streambed sediment were larger than equivalent concentrations in stream-water samples. The largest surface-water concentration of NPEO2 was $63 \mu \mathrm{g} / \mathrm{L}$, whereas the smallest estimated detection in streambed sediment was $83 \mu \mathrm{g} / \mathrm{kg}$ (sediment); laboratory reporting levels for all nonylphenol compounds in streambed sediment were $500 \mu \mathrm{g} / \mathrm{kg}$. Of the streambed-sediment samples that had NPEO2 detections, mean concentrations were more than two orders of magnitude larger than mean concentrations in base-flow samples, indicating that NPEO2 preferentially adsorbs to streambed sediment in Johnson County streams.

\section{Fragrances}

Fragrance compounds are often complex mixtures of chemicals, some of which can be toxic or carcinogenic (Fisher, 1998). Fragrance compounds with detections larger than laboratory reporting levels in Johnson County streambed-sediment samples included 3-methyl-1(H)-indole (Skatol), acetylhexamethyl-tetrahydro-naphthalene (AHTN), d-Limonene, hexahydro-hexamethyl-cyclopentabenzopyran (HHCB), and indole.

AHTN and HHCB are polycyclic synthetic musks used in a variety of personal care products, have been found to survive wastewater treatment, and have been found in both streambed sediment and fish tissue (Heberer and others, 2001). AHTN was detected in five streambed-sediment samples, two of which had concentrations larger than the laboratory reporting level $(50 \mu \mathrm{g} / \mathrm{kg})$; the samples were collected downstream from WWTF discharges at sites IN3a $(930 \mu \mathrm{g} / \mathrm{kg})$ and IN6 


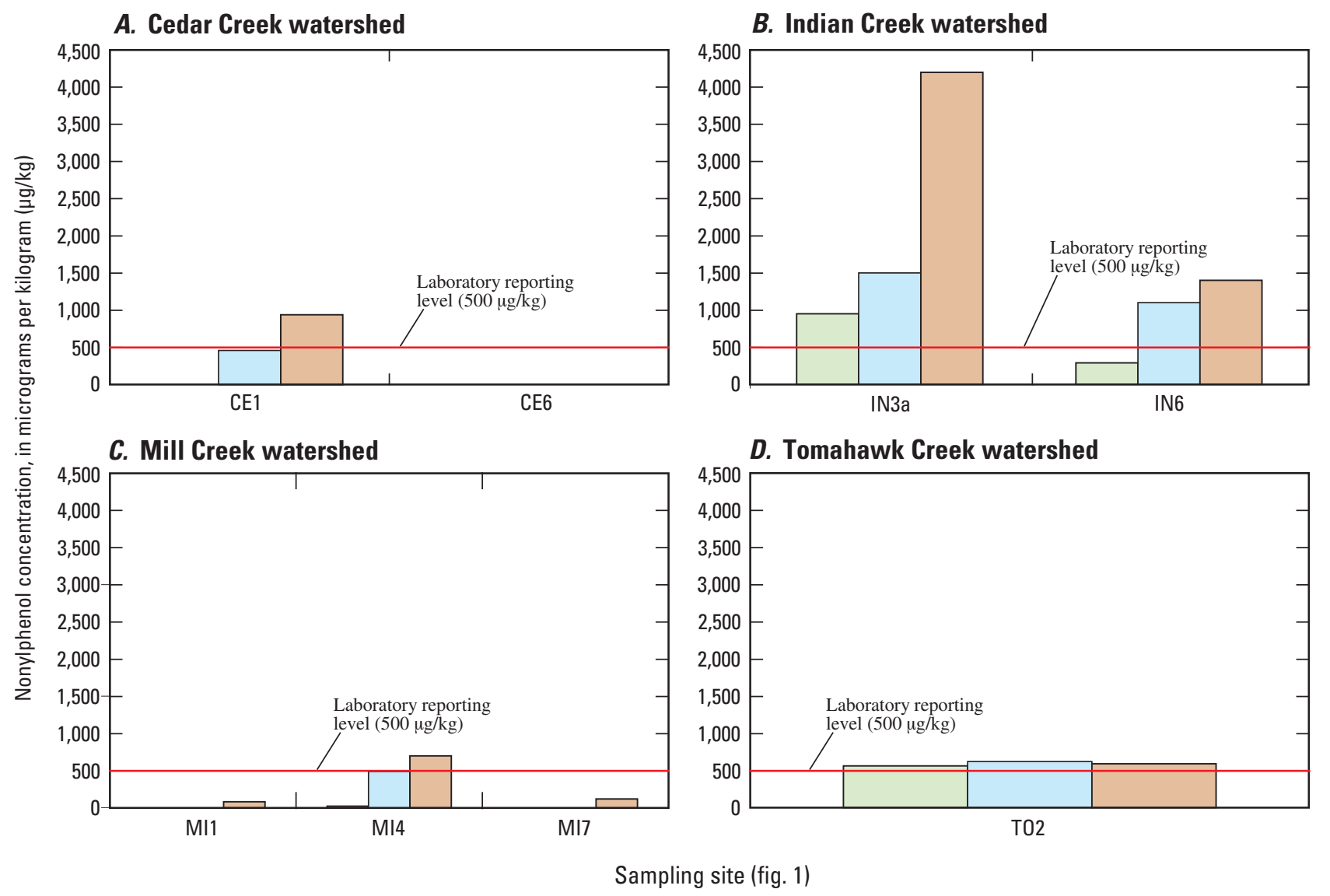

EXPLANATION

4-nonylphenol (4-NP)

Nonylphenol-ethoxylate (NPE01)

Nonylphenol-diethoxylate (NPE02)

Figure 35. Nonylphenol concentrations in streambed-sediment samples, March 31-April 3, 2003.

$(210 \mu \mathrm{g} / \mathrm{kg})$. HHCB was detected only in the sample from site IN3a $(180 \mu \mathrm{g} / \mathrm{kg})$, at a concentration larger than the laboratory reporting level $(50 \mu \mathrm{g} / \mathrm{kg}$ ) (table 22$)$.

$\mathrm{d}$-Limonene is a naturally occurring oil in citrus peels and other plants and commonly is used to provide a citrus flavor or fragrance in many foods and personal care products. d-Limonene is known to have toxic effects on some aquatic organisms and is expected to bind strongly to sediment (World Health Organization, 1998). d-Limonene was detected in samples from three sites; only the sample from site KI6b $(200 \mu \mathrm{g} / \mathrm{kg})$ had an estimated concentration larger than the laboratory reporting level $(50 \mu \mathrm{g} / \mathrm{kg})$.

Indole and skatol are in the class of indolic compounds and are used for a variety of applications, including cosmetics, pesticides, disinfectants, agrochemicals, and dyestuffs. Although there are little data on the fate and transport of these compounds or their potential effects on freshwater ecosystems, they have been found to be a nuisance due to their unpleasant odor $(\mathrm{Gu}$ and others, 2002). Indole was detected in streambed-sediment samples from all sites; the largest concentration was in the sample from site BI1 $(1,000 \mu \mathrm{g} / \mathrm{kg})$. Skatol was detected in samples from 13 of 15 sites; the largest concentration was in a sample from site TO2 $(1,000 \mu \mathrm{g} / \mathrm{kg})$. The detection of these compounds at a majority of sites suggests that they may be ubiquitous in streambed sediment throughout the county.

\section{Sterols and Stanols}

The sterols and stanols 3-beta-coprostanol (coprostanol), beta-sitosterol (sitosterol), beta-stigmastanol (stigmastanol), and cholesterol were detected in Johnson County streambedsediment samples. Coprostanol concentrations in Johnson County streambed sediment appear to be linked to WWTF discharges. Of the 10 detections, the largest concentrations were in samples from sites IN3a $(10,000 \mu \mathrm{g} / \mathrm{kg})$ and IN6 $(5,500 \mu \mathrm{g} / \mathrm{kg})$, sites nearest to WWTF discharges. Sitosterol and stigmastanol were not as linked to WWTF discharges. The 
Table 22. Concentrations of fragrance compounds in streambed-sediment samples collected from selected Johnson County streams, northeastern Kansas, March 31-April 3, 2003.

$[\mu \mathrm{g} / \mathrm{kg}$, micrograms per kilogram; LRL, laboratory reporting level; AHTN, acetyl-hexamethyl-tetrahydro-napthalene; HHCB, hexahydrohexamethyl-cyclopentabenzopyran; <, less than; E, estimated]

\begin{tabular}{|c|c|c|c|c|c|}
\hline \multirow{2}{*}{$\begin{array}{l}\text { Sampling site } \\
\text { identifier (fig. 1) }\end{array}$} & \multicolumn{5}{|c|}{ Concentration $(\mu \mathrm{g} / \mathrm{kg})$} \\
\hline & $\begin{array}{c}\text { 3-Methyl-1(H)-indole } \\
\text { (Skatol) }\end{array}$ & AHTN & d-Limonene & HHCB & Indole \\
\hline LRL & 50 & 50 & 50 & 50 & 50 \\
\hline$\overline{\mathrm{BI} 1}$ & 220 & $<50$ & E15 & $<50$ & 1,000 \\
\hline BL3 & 53 & $<50$ & $<50$ & $<50$ & 45 \\
\hline BL5 & 120 & $<50$ & $<50$ & $<50$ & 120 \\
\hline CA1 & 71 & $<50$ & $<50$ & $<50$ & 240 \\
\hline CE1 & 210 & E47 & $<50$ & $<50$ & 400 \\
\hline CE6 & E17 & E49 & $<50$ & $<50$ & 53 \\
\hline IN3a & 450 & 930 & $<50$ & 180 & 540 \\
\hline IN6 & 160 & 210 & $<50$ & $<50$ & 160 \\
\hline KI5 & 90 & $<50$ & $<50$ & $<50$ & 220 \\
\hline KI6b & $<50$ & $<50$ & E200 & $<50$ & 230 \\
\hline MI1 & E18 & $<50$ & $<50$ & $<50$ & 140 \\
\hline MI4 & 200 & E48 & $<50$ & $<50$ & 240 \\
\hline MI7 & 77 & $<50$ & $<50$ & $<50$ & 84 \\
\hline TO2 & 1,000 & $<50$ & E42 & $<50$ & 520 \\
\hline TU1 & $<50$ & $<50$ & $<50$ & $<50$ & 60 \\
\hline
\end{tabular}

largest concentrations of sitosterol were in samples from site BI1 $(28,000 \mu \mathrm{g} / \mathrm{kg})$ and site KI6b $(16,000 \mu \mathrm{g} / \mathrm{kg})$. Stigmastanol concentrations were the largest in samples from sites KI6b $(2,300 \mu \mathrm{g} / \mathrm{kg})$ and BI1 $(2,000 \mu \mathrm{g} / \mathrm{kg})$.

Cholesterol was detected in samples from every sediment site sampled, showing the ubiquitous nature of cholesterol sources. The largest concentrations were in samples from sites downstream from WWTF discharges, including sites IN3a $(30,000 \mu \mathrm{g} / \mathrm{kg})$, BI1 $(12,000 \mu \mathrm{g} / \mathrm{kg})$, and IN6 $(10,000 \mu \mathrm{g} / \mathrm{kg})$. Coprostanol/cholesterol ratios larger than 1 have been shown to be indicative of severe fecal contamination (Quéméneur and Marty, 1992; 1994). Coprostanol/cholesterol ratios were all less than 1; maximum ratios were in samples from sites downstream from WWTF discharges (sites IN3a, 0.33; IN6, 0.55; and MI4, 0.26).

\section{Other Compounds}

The "other" wastewater compound category includes five compounds with detections larger than laboratory reporting levels that did not fit within a broad grouping of detected compounds. These compounds were 9,10 -anthraquinone, carbazole, diethylhexyl phthalate, triclosan, and tris(2-butoxyethyl) phosphate. 9,10-anthraquinone was detected in samples from nine streambed-sediment sites; eight of the detections were larger than the laboratory reporting level $(50 \mu \mathrm{g} / \mathrm{kg})$. The largest concentrations were in samples from watersheds with urban land uses—sites IN3a (580 $\mu \mathrm{g} / \mathrm{kg})$, IN6 (590 $\mu \mathrm{g} / \mathrm{kg})$, MI1 $(110 \mu \mathrm{g} / \mathrm{kg})$, MI4 (110 $\mu \mathrm{g} / \mathrm{kg})$, MI7 (91 $\mu \mathrm{g} / \mathrm{kg})$, TO2 $(310 \mu \mathrm{g} / \mathrm{kg})$, and TU1 (120 $\mu \mathrm{g} / \mathrm{kg})$. Potential 9,10anthraquinone sources include application of bird repellents and (or) degradation of anthracene to 9,10-anthraquinone (McKinney and others, 1999; U.S. Environmental Protection Agency, 2005b).

Eight detections were made of carbazole in Johnson County streambed-sediment samples; four of the detections were larger than the laboratory reporting level $(50 \mu \mathrm{g} / \mathrm{kg})$. Carbazole concentrations were largest in samples collected downstream from WWTF discharges and at urban sites. The largest concentrations were in samples from sites IN $3 \mathrm{a}(310 \mu \mathrm{g} / \mathrm{kg})$ and IN6 $(300 \mu \mathrm{g} / \mathrm{kg})$. Other concentrations larger than the laboratory reporting level were in samples from site TO2 $(140 \mu \mathrm{g} / \mathrm{kg})$ and site TU1 $(55 \mu \mathrm{g} / \mathrm{kg})$, likely due to runoff from urban roadways and parking lots.

Diethylhexyl phthalate (DEHP) is a phthalate ester, a compound commonly used to increase the flexibility of plastics, and is known to adsorb to solids (Zhou and Liu, 2000). DEHP was detected in samples from eight sites, all at concentrations larger than the laboratory reporting level $(100 \mu \mathrm{g} / \mathrm{kg})$. DEHP concentrations were largest in samples from urban sites downstream 
from WWTF discharges, suggesting that the primary sources of this compound are WWTF discharge. The largest DEHP concentrations were in samples from site IN3a $(2,700 \mu \mathrm{g} / \mathrm{kg})$ and site IN6 $(1,800 \mu \mathrm{g} / \mathrm{kg})$.

Triclosan is an antibacterial agent used in a variety of cosmetic products. It is only slightly soluble in water and generally is well removed by WWTFs (Sabaliunas and others, 2003). Six detections of triclosan were made in Johnson County streambed-sediment samples; samples collected downstream from WWTFs at sites IN6 $(160 \mu \mathrm{g} / \mathrm{kg})$ and IN3a $(140 \mu \mathrm{g} / \mathrm{kg})$ had concentrations larger than the laboratory reporting level $(50 \mu \mathrm{g} / \mathrm{kg})$.

Tris(2-butoxyethyl) phosphate (TBEP) is in a class of plasticizers often used in floor polish and is thought to be moderately toxic to aquatic organisms (World Health Organization, 2000). TBEP was detected in 2 of 15 streambed-sediment samples (site CE6, $300 \mu \mathrm{g} / \mathrm{kg}$; site IN6, $150 \mu \mathrm{g} / \mathrm{kg}$ ) at concentrations larger than the laboratory reporting level $(100 \mu \mathrm{g} / \mathrm{kg})$.

\section{Pharmaceutical Compounds}

Twenty-two pharmaceutical compounds were analyzed in all Johnson County streambed-sediment samples (table 6). Interpretation of pharmaceutical data is limited primarily because small spike recoveries indicate that many pharmaceutical compounds likely were underreported in streambedsediment samples. The number of pharmaceutical detections (fig. 36A) and total pharmaceutical concentrations (fig. 36B) are associated with WWTF discharge. Sites downstream from WWTF discharges (sites BI1, CE6, IN3a, IN6, KI5, KI6b, MI4, MI7) averaged $84 \mu \mathrm{g} / \mathrm{kg}$ for all pharmaceutical compounds concentrations combined, whereas sites upstream from WWTF discharges (sites BL3, BL5, CA1, CE1, MI1 TO2, TU1) averaged $13 \mu \mathrm{g} / \mathrm{kg}$ of combined pharmaceutical concentrations. Caffeine was the most frequently detected pharmaceutical compound (detected at 12 of 15 sites) and was found in the largest concentrations of any pharmaceutical compounds. Caffeine was found in the largest concentrations at sites MI7 (54 $\mu \mathrm{g} / \mathrm{kg})$, MI4 (46 $\mu \mathrm{g} / \mathrm{kg})$, and CE6 (42 $\mu \mathrm{g} / \mathrm{kg})$; however, caffeine was not detected at site IN3a, the site with the largest total concentration of pharmaceutical compounds. Two other compoundsdiphenhydramine (antihistamine) and cotinine (nicotine metabolite)—were detected in a majority of Johnson County streambed-sediment samples.

Site IN3a is located closest to a WWTF discharge and had the largest total concentrations of pharmaceutical compounds. Site IN3a had the largest total concentration of diphenhydramine (antihistamine) $(134 \mu \mathrm{g} / \mathrm{kg})$ along with site IN6 $(27 \mu \mathrm{g} / \mathrm{kg})$. Indian Creek sites also had the largest concentrations of trimethoprim (antimicrobial) (site IN3a, $22 \mu \mathrm{g} / \mathrm{kg}$; site IN6, $15 \mu \mathrm{g} / \mathrm{kg}$ ) and cimetidine (antacid) (site IN3a, $5.1 \mu \mathrm{g} / \mathrm{kg}$; site IN6, $2.6 \mu \mathrm{g} / \mathrm{kg}$ ). Acetaminophen (antipyretic) was detected in larger concentrations at sites MI4 $(49 \mu \mathrm{g} / \mathrm{kg})$, CE6 (39 $\mu \mathrm{g} / \mathrm{kg})$, MI7 (24 $\mu \mathrm{g} / \mathrm{kg})$, and CE1 (24 $\mu \mathrm{g} / \mathrm{kg})$. Site TU1 had the most detections (six) of pharmaceutical compounds, and site CE1 had the largest total concentration of pharmaceutical compounds (42 $\mu \mathrm{g} / \mathrm{kg}$ ) of sites upstream from WWTF discharges.

\section{Summary of Stream-Water and Streambed- Sediment-Quality Data by Watershed}

The following sections summarize stream-water and streambed-sediment-quality data by each watershed sampled from October 2002 through June 2004 in Johnson County, Kansas.

\section{Big Bull Creek Watershed}

Two base-flow samples and one streambed-sediment sample were collected at site BI1 in the Big Bull Creek watershed (table 2). Site BI1 was located downstream from two municipal WWTFs; a majority of upstream land use is devoted to agriculture (primarily cropland and grassland). The Big Bull Creek watershed is partially contained within Johnson County; the creek flows downstream into Miami County, Kansas (fig. 1). Base-flow concentrations of dissolved solids, nutrients, and indicator bacteria were among the smallest in the county and, generally, were consistent with other base-flow samples downstream from, but at a distance from, WWTF discharge. The KDHE chronic aquatic-life criterion for atrazine $(3.0 \mu \mathrm{g} / \mathrm{L})$ was exceeded during the July synoptic survey (3.5 $\mu \mathrm{g} / \mathrm{L})$, which is generally consistent with results determined by Putnam (1997). A mean of 11 wastewater compounds were detected in samples collected during base-flow surveys; the majority of detections were at concentrations less than laboratory reporting levels.

The one streambed-sediment sample from the Big Bull Creek watershed contained the largest total organic carbon, nitrogen, and phosphorus concentrations of all Johnson County streambed-sediment samples. The Big Bull Creek streambedsediment sample did not contain any pesticides but had the second largest total concentration of wastewater compounds, 94 percent of which consisted of indole, para-cresol, phenol, sterols, and stanols. Neither PAHs or nonylphenol compounds were detected in the streambed-sediment sample. The streambed-sediment sample from site BI1 had among the smallest concentrations of pharmaceutical compounds of samples from sites downstream from WWTF discharges. Although base-flow concentrations of contaminants generally were small, site BI1 represents a depositional zone for nutrients, as well as wastewater compounds generally associated with agriculture and plant matter.

\section{Blue River Watershed}

Seven base-flow samples (two collected during the first synoptic survey and five collected during the second survey), eight stormflow samples (from site BL5), and two streambedsediment samples (from sites BL3 and BL5) were collected in the Blue River watershed. The Blue River watershed is the 


\section{A. Number of detections}

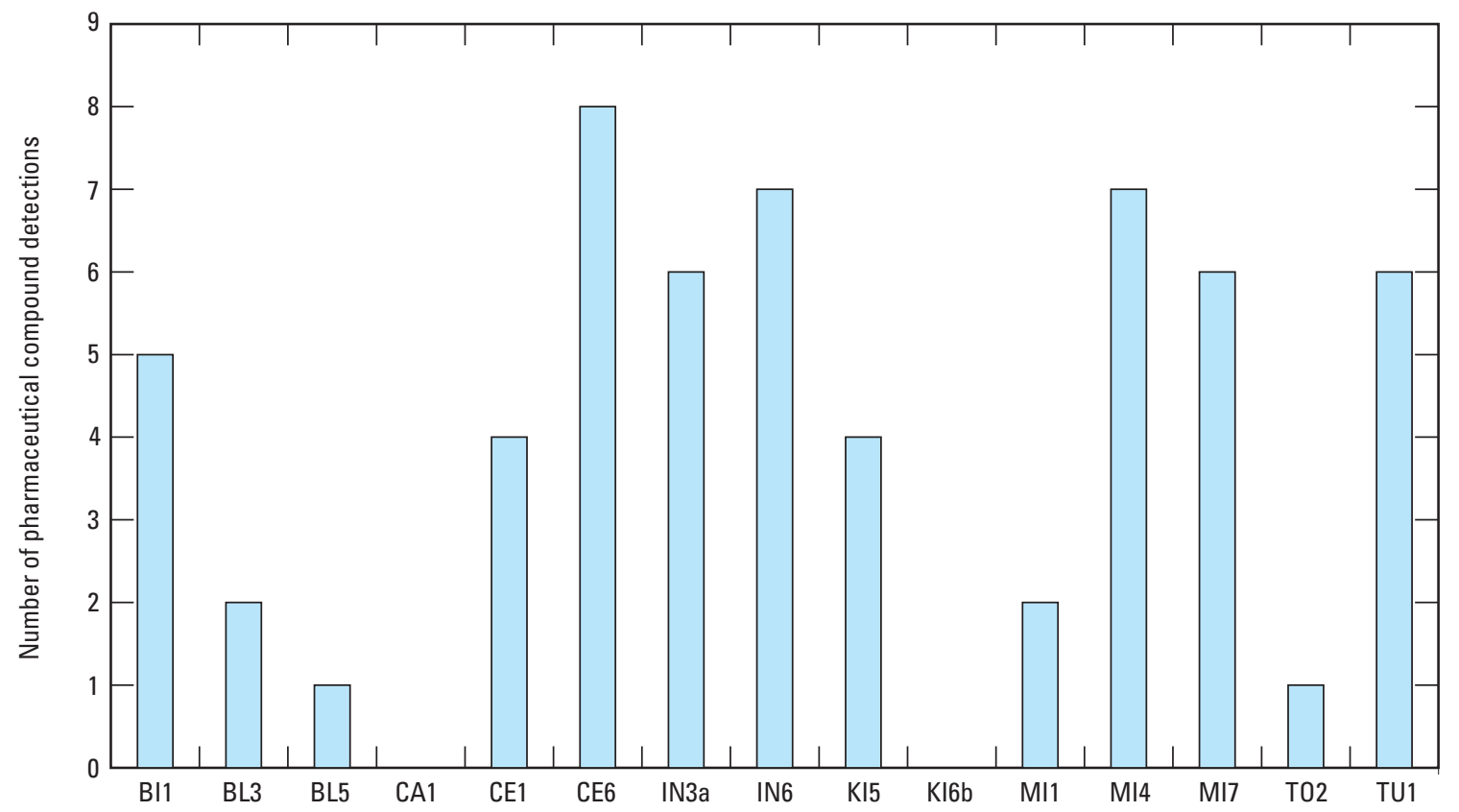

B. Total concentrations

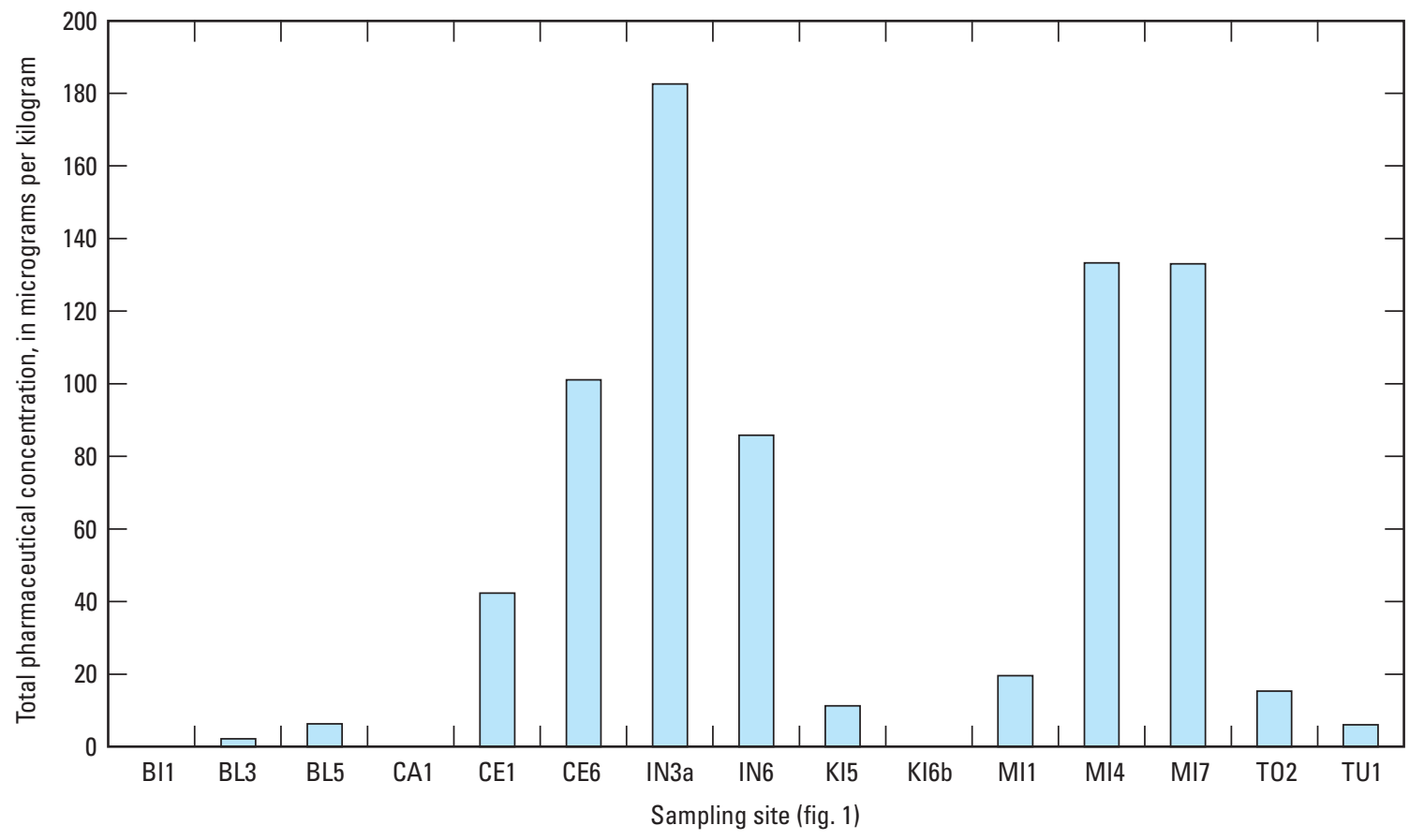

Figure 36. (A) Number of pharmaceutical compound detections and $(B)$ total concentrations in streambedsediment samples, March 31-April 3, 2003. 
largest watershed in Johnson County; the river flows downstream into Jackson County, Missouri (fig. 1). Land use is primarily undeveloped (61 percent), but it is becoming more urban, especially in the northern and downstream parts of the watershed. The stormflow site BL5 is located upstream from the WWTF discharge (site BL6); thus, it is noted that stormflow concentrations and loads from this site do not include any sanitary sewer overflows from site BL6.

Similar to other large watersheds with WWTF discharges, nearly 100 percent of streamflow at the farthest downstream site during base-flow conditions was contributed by WWTF discharge (fig. 7, site BL7) near the Kansas-Missouri State line. Dissolved-solids concentrations were larger in samples from site BL6 (Blue River Main Number 1 WWTF) than in any other stream-water samples collected in the watershed. Suspendedsediment concentrations and loads were much larger in stormflow samples than in base-flow samples.

The largest total nitrogen and phosphorus concentrations in all stream-water samples were from WWTF discharge (site BL6) during base-flow conditions. Nitrate and orthophosphorus were the dominant nutrient species in these samples. Nutrient concentrations and loads generally increased with increasing streamflow conditions at site BL5 (upstream from the WWTF discharge). Indicator bacteria densities and loads were larger during stormflow conditions, indicating that nonpoint sources are the largest contributors of indicator bacteria upstream from site BL5.

Many pesticide compounds were detected at the largest concentrations in spring stormflow samples from the Blue River watershed, whereas the majority of wastewater compound detections and largest concentrations were at, or downstream from, the Blue River Main Number 1 WWTF (site BL6) during base-flow conditions. The majority of the pesticide loads likely were contributed during stormflow conditions soon after pesticide application in the spring and summer months. Activated-sludge secondary treatment processes likely were responsible for improved removal of wastewater compounds found in larger concentrations in other Johnson County WWTF discharges. There were no detections of nonylphenol compounds greater than laboratory reporting levels in base-flow samples; the largest concentrations and loads of nonylphenoldiethoxylate were in stormflow samples. Like other Johnson County streams during base-flow conditions, pharmaceutical detections were largest at and downstream from WWTF discharges in samples from sites BL6 and BL7.

Streambed sediment in samples from sites BL3 and BL5 generally had among the smallest mean concentrations of nutrients, trace elements, and bacteria densities because of site locations upstream from WWTF discharges and downstream from mostly nonurban land uses. Blue River streambed-sediment samples also had no pesticide detections and had among the fewest wastewater and pharmaceutical compound detections in the county. Along with Captain and Kill Creek samples, Blue River streambed-sediment samples represented the leastaffected sediment-quality condition in Johnson County.

\section{Brush Creek Watershed}

Four base-flow samples were collected at two Brush Creek sites (BR1 and BR2); no stormflow or streambed-sediment samples were collected. Brush Creek flows downstream into Jackson County, Missouri, eventually entering the Blue River (fig. 1). Site BR1 is downstream from the upper Brush Creek watershed; the downstream site, BR2, includes the upper part of Brush Creek and the entire Rock Creek watershed. Sampling sites were downstream from mostly residential land uses, some commercial land uses, and among the largest percentage of impervious surface in Johnson County.

Brush Creek base-flow samples had the smallest mean dissolved-solids concentrations in the county $(272 \mathrm{mg} / \mathrm{L})$ and among the smallest total nitrogen and total phosphorus concentrations. Mean fecal coliform $(580 \mathrm{col} / 100 \mathrm{~mL})$ and E. coli $(250 \mathrm{col} / 100 \mathrm{~mL})$ densities in Brush Creek samples were more than double that of Johnson County median values in base-flow samples (200 col/100 mL, fecal coliform; $120 \mathrm{col} / 100 \mathrm{~mL}$, E. coli). Base-flow samples from Brush Creek sites generally had fewer detections and smaller concentrations of pesticide and wastewater compounds when compared with all Johnson County base-flow samples. However, when compared to samples from other sites upstream from WWTFs, samples from Brush Creek sites had among the largest number of pesticide and wastewater compound detections, averaged the largest diazinon concentrations, and samples from two of seven upstream sites had caffeine and tri(2-butoxyethyl) phosphate detections larger than laboratory reporting levels. Although streamflow conditions and concentrations of many contaminants were small during base-flow conditions, the urban nature of this watershed lends to larger densities of bacteria and concentrations of some wastewater compounds compared to other base-flow samples collected upstream from WWTF discharges. Potential sources of these constituents could include leaking sewage lines, golf courses, nonpoint application or spillage, domestic or wild animals, or other sources.

\section{Captain Creek Watershed}

Only one streambed-sediment sample was collected from Captain Creek because of a lack of measurable streamflow at site CA1 during base-flow synoptic surveys. No stormflow samples were collected in this watershed. The Captain Creek watershed is contained within both Douglas and Johnson County, Kansas; the creek flows downstream into the Kansas River (fig. 1). Site CA1 has predominantly nonurban land use upstream, along with the inactive Sunflower Army Ammunition Plant.

The Captain Creek streambed-sediment samples had among the smallest concentrations of nutrients, trace elements, and similar densities of $E$. coli relative to streambed sediment in samples from other Johnson County sites. One pesticide was detected ( $1 \mathrm{mg} / \mathrm{kg}$ of dieldrin) in the sample from site CA1. The sample from site CA1 had among the fewest detections and 
smallest concentrations of wastewater compounds among Johnson County streambed-sediment samples. No pharmaceutical compounds were detected in the Captain Creek streambedsediment sample. Along with Blue River and Kill Creek samples, the Captain Creek streambed-sediment sample represented the least-affected sediment-quality condition in Johnson County.

\section{Cedar Creek Watershed}

Thirteen base-flow samples (four collected during the first synoptic survey, six collected during the second survey, and three additional base-flow samples collected at site CE6), six stormflow samples (site CE6), and two streambed-sediment samples (sites CE1 and CE6) were collected in the Cedar Creek watershed. The Cedar Creek watershed is contained entirely within Johnson County; the creek flows downstream into the Kansas River (fig. 1). Cedar Creek has a primarily nonurban watershed and also has the largest percentage of land area devoted to impoundments (table 4), including Cedar Lake and Lake Olathe. More than 99 percent of the streamflow from Cedar Creek entering the Kansas River during base-flow sample collection was contributed by WWTF discharge (fig. 6 , site CE6).

Base-flow samples collected at site CE1 (upstream from WWTF discharge site CE3) had among the largest concentrations of dissolved solids, calcium, magnesium, sodium, and sulfate of base-flow samples collected from all Johnson County streams. These concentrations likely are due to bedrock dissolution from large rock quarries in the watershed upstream from site CE1. Suspended-sediment concentrations and estimated loads were much larger in stormflow samples than in baseflow samples.

The largest total nitrogen and phosphorus concentrations in all stream-water samples were from WWTF discharge (site CE3) during base-flow conditions (as in all other watersheds with sampled WWTF discharges) and from a base-flow sample collected from site CE6 on February 14, 2003. Although this is classified as a base-flow sample, streamflow conditions were elevated above normal base-flow conditions due to a 1.3-in. rainfall from February 13 through 14 (Overland Park Stormwatch, 2004), thus nutrient concentrations may be affected by nonpoint-source and (or) sanitary sewer overflows. Nitrate and orthophosphorus were the dominant nutrient species in base-flow samples. Mean nutrient concentrations were similar between base- and stormflow samples; however, instantaneous nutrient loads increased with streamflow conditions at site CE6. Indicator bacteria densities and loads were larger during stormflow conditions, indicating that nonpoint sources and (or) sanitary sewer overflows are likely the largest contributors of indicator bacteria upstream from site CE6.

Several pesticides compounds, including carbaryl $(0.22 \mu \mathrm{g} / \mathrm{L})$, diazinon $(0.31 \mu \mathrm{g} / \mathrm{L})$, and simazine $(0.56 \mu \mathrm{g} / \mathrm{L})$, were detected in among the largest concentrations of all Johnson County stream-water samples in a stormflow sample collected at site CE6 on June 23, 2003. These large concentrations likely were due to seasonal pesticide application in agricultural areas or from lawn and golf course application from small developments upstream from site CE6. Atrazine $(0.72 \mu \mathrm{g} / \mathrm{L}$, July 2003) was found in the second-largest concentration of all Johnson County base-flow samples in a sample from site CE1; it is not known why this site had larger concentrations than other base-flow samples downstream from agricultural land uses. Pesticide and wastewater compounds were detected consistently in samples from the Cedar Creek watershed and typically were found in samples from both site CE1 upstream from WWTF discharge and downstream from the Cedar Creek WWTF at site CE3. Samples from site CE1 had the largest mean total concentrations of wastewater compounds $(18.7 \mu \mathrm{g} / \mathrm{L})$ compared to all other sites upstream from WWTF discharge, primarily due to large detergent metabolite concentrations. This site also had the largest NPEO2 concentrations of all Cedar Creek stream-water samples. Rock-quarry operations and (or) failing septic systems upstream may be the source of these concentrations. Samples from site CE1 had among the fewest detections and smallest concentrations of pharmaceutical compounds than all other site samples.

The streambed-sediment sample from site CE1 (along with sites BI1 and IN6) had the most (seven) trace elements concentrations that were larger than USEPA threshold effect levels. It also had nitrogen concentrations equal to the median for Johnson County streambed-sediment samples $(1,600 \mathrm{mg} / \mathrm{kg})$, but had phosphorus concentrations $(1,900 \mathrm{mg} / \mathrm{kg})$ nearly double that for median Johnson County streambed-sediment concentrations $(1,000 \mathrm{mg} / \mathrm{kg})$. The sample from site CE1 had indicator bacteria densities larger than median values for Johnson County streambed sediment, had the most detections (22) and among the largest total concentration of wastewater compounds $(17.9 \mathrm{mg} / \mathrm{kg})$, and the largest total concentration of pharmaceutical compounds $(42 \mu \mathrm{g} / \mathrm{kg})$ among sites upstream from WWTF discharges. Sources of these elevated contaminant concentrations could include rock quarry operations or failing septic systems upstream from site CE1.

The streambed-sediment sample from site CE6 had fewer trace element concentrations larger than USEPA sediment guidelines (four), a nitrogen concentration $(1,200 \mathrm{mg} / \mathrm{kg}$ ) smaller than the Johnson County median concentration, and a phosphorus concentration $(1,100 \mathrm{mg} / \mathrm{kg})$ slightly larger than the median concentration for Johnson County. Indicator bacteria densities in the sample from site CE6 were less than Johnson County median values, and the sample from site CE6 also had among the fewest wastewater compound detections in Johnson County streambed sediment (10 detections). However, streambed sediment from site CE6 had the most detections (eight) and among the largest concentrations of pharmaceutical compounds $(101 \mu \mathrm{g} / \mathrm{kg})$. Neither Cedar Creek sampling site had pesticide detections in streambed-sediment samples. 


\section{Dykes Branch Watershed}

Two base-flow samples were collected from the Dykes Branch watershed, a small, predominantly residential watershed located on the east side of Johnson County. Dykes Branch is partially contained within Johnson County, entering the Blue River through Indian Creek in Jackson County, Missouri (fig. 1).

Dissolved-solids and nutrient concentrations were less than base-flow median values for the county, and the mean E. coli density $(200 \mathrm{col} / 100 \mathrm{~mL})$ was larger than the Johnson County median value $(120 \mathrm{col} / 100 \mathrm{~mL})$. Stream-water samples from Dykes Branch had among the fewest detections and smallest concentrations of pesticide and wastewater compounds; however, site DY1 was the only site upstream from WWTF discharges with samples that contained DEET concentrations equal to or larger than the laboratory reporting level and was one of six sites upstream from WWTFs to have samples with caffeine detections larger than the laboratory reporting level. Similar to other urban sites upstream from WWTF discharges, samples from Dykes Branch Creek had smaller concentrations of many water-quality constituents but had larger concentrations and more detections of bacteria and some wastewater compounds than samples from sites in nonurban watersheds. Potential sources of these water-quality contaminants could include leaking sewage lines, nonpoint application or spillage, domestic or wild animals, or other sources.

\section{Indian and Tomahawk Creek Watersheds}

Twelve base-flow samples (five collected during the first synoptic survey, six collected during the second, and one additional at site IN6), eight stormflow samples (collected from site IN6), and two streambed-sediment samples (sites IN3a and IN6) were collected from the Indian Creek watershed. The Tomahawk Creek watershed is upstream from sites IN5 and IN6; six base-flow samples and one streambed-sediment sample (site TO2) from Tomahawk Creek are included in this analysis. The Indian Creek watershed is partially contained within Johnson County; the creek flows downstream into the Blue River in Jackson County, Missouri (fig. 1). The Tomahawk Creek watershed is entirely contained within Johnson County; the creek discharges into Indian Creek at site IN5 (fig. 1). In combination, the Indian and Tomahawk Creek watersheds are the second largest watershed in the county and consist of mostly residential and commercial land uses. Ninety percent of streamflow exiting Johnson County during base-flow conditions was contributed by wastewater discharges (fig. 7, sites IN3 and TO3).

A stormflow sample from site IN6 on January 26, 2004, had the largest dissolved-solids $(2,000 \mathrm{mg} / \mathrm{L})$ and chloride $(1,000 \mathrm{mg} / \mathrm{L})$ concentrations detected in the county, likely due to road-salt application. Other than this sample, and similar to other watersheds with WWTF discharges, dissolved-solids concentrations were largest during base-flow sample collection at
WWTFs (sites IN3 and TO3). Suspended-sediment concentrations and loads were larger in stormflow than in base-flow samples.

The largest total nitrogen and phosphorus concentrations in all stream-water samples were from WWTF discharges during base-flow conditions (sites IN3 and TO3). Nitrate and orthophosphorus were the dominant nutrient species in WWTF samples; however, ammonia concentrations were also among the largest in Johnson County stream-water samples at site TO3. Nutrient concentrations were largest during base-flow conditions at site IN6 due to proximity to WWTF discharge site TO3. Nutrient loads increased with increasing streamflow conditions, which may indicate nonpoint sources and (or) sanitary sewer overflows. Site IN1 had among the largest base-flow indicator bacteria densities of sites upstream from WWTF discharges; however, indicator bacteria densities and loads were larger during stormflow conditions, indicating that nonpoint sources and (or) sanitary sewer overflows are likely the largest contributors of indicator bacteria in these watersheds.

Almost all other pesticide and wastewater compounds found in Indian Creek recorded the most detections and largest concentrations downstream from WWTF discharges. Samples from the Tomahawk Creek WWTF (site TO3) had among the most wastewater compounds detected in the county (51 in both base-flow samples), likely due to less-effective trickling-filter secondary treatment processes in removing these constituents. Caffeine $(12 \mu \mathrm{g} / \mathrm{L})$, cholesterol $(10 \mu \mathrm{g} / \mathrm{L})$, coprostanol $(5 \mu \mathrm{g} / \mathrm{L})$, and phenol $(1.8 \mu \mathrm{g} / \mathrm{L})$ were detected at the largest concentrations in Johnson County stream water in samples from site TO3. Base-flow samples from site TO3 had the largest concentrations of NPEO2 in the watershed. Samples at and downstream from the activated-sludge WWTF (site IN3) had among the most (along with trickling-filter WWTF site TU3) detections of pharmaceutical compounds compared to samples from all other sites but had smaller total concentrations of pharmaceutical compounds than did samples from site TU3 (fig. 29).

Streambed-sediment samples from sites IN3a and IN6 had among the most detections and largest concentrations of nutrients, trace elements, bacteria, pesticides, and wastewater and pharmaceutical compounds in Johnson County. These sites were the closest to WWTF discharges in the county, which likely were the cause of increased contaminant deposition. Samples from site IN3a had the largest silver concentrations in Johnson County sediment samples $(2.3 \mathrm{mg} / \mathrm{kg})$, larger than USEPA PEL guidelines, likely because of the use of silver in photographic processing. Nitrogen and phosphorus concentrations also were among the largest in the county because of WWTF discharges containing large nutrient concentrations. Streambed-sediment samples from site IN6 had among the largest concentrations of chlordane, dieldrin, and DDT in Johnson County. These constituents have a high adsorptive capacity and are very persistent in the environment; thus, the large streambed-sediment concentrations may be due to residential and (or) agricultural application prior to USEPA bans of these compounds. PAH compounds were detected in the largest concentrations in samples from sites IN3a, IN6, and TO2. PAHs 
likely were contributed by oil spills and (or) automobile emissions. Although samples from sites IN3a and IN6 had among the largest number of total detections and concentrations of wastewater and pharmaceutical compounds in the county, samples from site TO2 also had among the most wastewater compound detections (24), despite being upstream from WWTF discharges.

\section{Kill Creek Watershed}

Ten base-flow samples (three collected during the first synoptic survey, seven collected during the second), seven stormflow samples (site KI6b), and two streambed-sediment samples (sites KI5 and KI6b) were collected in the Kill Creek watershed. The Kill Creek watershed is entirely contained within Johnson County; the creek flows downstream into the Kansas River (fig. 1). Kill Creek drains a primarily agricultural watershed, which also contains a part of the inactive Sunflower Army Ammunition Plant. Similar to other Johnson County watersheds, greater than 99 percent of flow entering the Kansas River from Kill Creek (fig. 7, site KI7) during base-flow sample collection was contributed by wastewater discharge (site KI2).

Base-flow samples from the WWTF (site KI2) had slightly larger dissolved-solids concentrations than did other Kill Creek stream-water samples. Suspended-sediment concentrations and loads were much larger during stormflow than during base-flow conditions.

The largest total nitrogen concentrations in all streamwater samples were from WWTF discharge (site KI2) during base-flow conditions on November 4, 2002, because the facility was new and bacterial processes were not mature in the activated sludge (E. Hack, Johnson County Wastewater and Public Works, written commun., 2005). Total phosphorus concentrations were largest at this site during both base-flow synoptic surveys. Nitrate and orthophosphorus were the dominant nutrient species in WWTF samples. Nutrient concentrations and loads generally increased with increasing streamflow conditions at site KI6b. Indicator bacteria densities and loads were larger during stormflow conditions, indicating that nonpoint sources and (or) sanitary sewer overflows were likely the largest contributors of indicator bacteria upstream from site KI6b.

The most pesticide and wastewater compound detections (as well as the largest concentrations) occurred in base-flow samples collected from the Kill Creek WWTF (site KI2). However, pesticide and wastewater compound detections generally were fewer, and of a lesser magnitude, than in base-flow samples from other large watersheds with WWTFs. The largest NPEO2 concentration was during stormflow conditions at site KI6b, likely indicating nonpoint sources and (or) sanitary sewer overflows were the largest contributors of detergent surfactants in the Kill Creek watershed.

Streambed-sediment samples from sites KI5 and KI6b had among the smallest concentrations of nutrients, trace elements, bacteria, and wastewater and pharmaceutical compounds of any streambed-sediment samples in the county. Samples from Kill
Creek sites had only one detection of trace elements larger than USEPA TEL guidelines (nickel); samples from all other Johnson County sites also exceeded this guideline. No pesticide detections occurred in either Kill Creek streambed-sediment sample. Along with Blue River and Captain Creek samples, Kill Creek streambed-sediment samples represented the leastaffected sediment-quality condition in Johnson County.

\section{Little Bull Creek Watershed}

Two base-flow samples were collected at site LI1, located where Little Bull Creek exits the southern end of Johnson County (fig. 1). The Little Bull Creek watershed is only partially contained within Johnson County; the creek flows downstream into Miami County (fig. 1). Land use in the Little Bull Creek watershed is primarily agricultural, largely consisting of cropland and grassland. One WWTF is located upstream from site LI1, at the New Century Air Center, in the northern part of the watershed.

Dissolved-solids and nutrient concentrations in base-flow samples were among the smallest in the county, whereas suspended-sediment concentrations were the largest of any base-flow samples collected during the second base-flow synoptic survey $(220 \mathrm{mg} / \mathrm{L})$. Spraying of gravel roads in the area by maintenance crews to control dust likely contributed to large suspended-sediment concentrations. Mean indicator bacteria densities were larger than Johnson County median levels, whereas Little Bull Creek base-flow samples had among the fewest detections of pesticide and wastewater compounds, with very few detections larger than laboratory reporting levels.

\section{Mill Creek Watershed}

Fifteen base-flow samples (six collected during the first synoptic survey, seven collected during the second, and two additional samples from site MI7), 10 stormflow samples (site MI7), and three streambed-sediment samples (sites MI1, MI4, and MI7) were collected from the Mill Creek watershed. The Mill Creek watershed is entirely contained within Johnson County; the creek flows downstream into the Kansas River (fig. 1). Mill Creek is an urbanizing watershed; land use is divided between residential, commercial, and agricultural land uses. Unlike other watersheds with WWTF discharges, less than 20 percent of the flow entering the Kansas River from Mill Creek (fig. 7, site MI7) was contributed by wastewater discharge (site MI2) during base-flow sample collection, likely due to a small amount of rain that fell during base-flow sampling in the watershed.

A stormflow sample collected on March 12, 2003, had the largest dissolved-solids concentrations $(960 \mathrm{mg} / \mathrm{L})$ of any Mill Creek sample, likely the result of road-salt application. Other than stormflow samples affected by road-salt application, baseflow samples at and downstream from the WWTF discharge (sites MI2 and MI3) had the largest dissolved-solids concentrations. Suspended-sediment concentrations and loads 
were much larger in stormflow samples than in base-flow samples.

The largest total nitrogen and phosphorus concentrations in all stream-water samples were from WWTF discharge (site MI2) during base-flow conditions. Nitrate and orthophosphorus were the dominant nutrient species in WWTF samples. Nutrient concentrations and loads were larger in stormflow than base-flow samples at site MI7 (upstream from the WWTF discharge). Sites MI1 and MI5 had among the largest indicator bacteria densities of sites upstream from WWTF discharges, and site MI2 had among the largest indicator bacteria densities of WWTF discharges in Johnson County base-flow samples. However, indicator bacteria densities and loads were largest during stormflow conditions, indicating that nonpoint sources and (or) sanitary sewer overflows are likely the largest contributors of indicator bacteria in the Mill Creek watershed.

The most detections and largest concentrations of pesticide and wastewater compounds in Mill Creek stream-water samples generally occurred in samples collected at, or immediately downstream from, WWTF discharge site MI2, although samples from site MI1 had the largest concentrations of phenol $(0.6 \mu \mathrm{g} / \mathrm{L})$ and tri(2-butoxyethyl) phosphate $(7.4 \mu \mathrm{g} / \mathrm{L})$ compared with other samples collected in the Mill Creek watershed. NPEO2 concentrations at site MI2 were the second largest in Johnson County stream-water samples. Pesticide and wastewater compound concentrations were similar in base- and stormflow samples from site MI7.

Streambed-sediment samples from three sites showed substantial differences in contaminant concentrations. The sample from site MI1 had the largest concentrations of lead $(73 \mathrm{mg} / \mathrm{kg})$, zinc $(230 \mathrm{mg} / \mathrm{kg})$, total chlordane $(26 \mu \mathrm{g} / \mathrm{kg})$, and total DDT $(16 \mu \mathrm{g} / \mathrm{kg})$ of all Johnson County streambed-sediment samples. Phosphorus (1,500 mg/kg), fecal coliform (380 col/GDW), E. coli $(280 \mathrm{col} / \mathrm{GDW})$, and total PAHs were detected in the largest concentrations and densities in the sample from site MI1 compared with all Mill Creek samples. Elevated concentrations of contaminants in streambed sediment from site MI1 may be due to runoff from urban land uses upstream, municipal sewer leakages, or industrial land uses upstream. Site MI4 is downstream from the Harold Street WWTF (site MI2, fig. 1), and the streambed-sediment sample from this site had the largest nitrogen concentration $(1,900 \mathrm{mg} / \mathrm{kg})$, the most wastewater compound detections (24), and the largest total concentration of wastewater compounds of all other Mill Creek samples. Streambed sediment in the sample from site MI7 had among the smallest concentrations of many constituents compared with all other Johnson County streambed-sediment samples. PAHs were detected in samples from all Mill Creek streambedsediment sites, likely due to sources from automobile usage. Samples from sites MI4 and MI7 had among the most detections (site MI4, seven detections; site MI7, six detections) and total concentrations (site MI4, $133 \mu \mathrm{g} / \mathrm{kg}$; site MI7, $133 \mu \mathrm{g} / \mathrm{kg}$ ) of pharmaceutical compounds in Johnson County streambed sediment, likely due to WWTF discharge upstream.

\section{Turkey Creek Watershed}

Six base-flow samples (three collected during the first synoptic survey and three collected during the second), four stormflow samples (site TU1), and one streambed-sediment sample (site TU1) were collected from the Turkey Creek watershed.

The Turkey Creek watershed is only paritally contained within Johnson County; the creek flows downstream into Wyandotte County, Kansas (fig. 1). Turkey Creek is a small watershed with mostly residential and commercial land uses and the largest percentage of impervious surface in the county. Nearly 100 percent of streamflow leaving the county through Turkey Creek during base-flow sample collection was contributed by WWTF discharges (fig. 7, site TU3).

The largest dissolved-solids concentrations were found in base-flow samples of WWTF discharge from site TU3 (mean = $595 \mathrm{mg} / \mathrm{L}$ ). Suspended-sediment concentrations and loads were much larger in stormflow samples than in base-flow samples.

The largest total nitrogen and phosphorus concentrations in all Turkey Creek stream-water samples were from WWTF discharge (site TU3) during base-flow conditions. Nitrate and orthophosphorus were the dominant nutrient species in WWTF samples; samples from this site also had the largest mean concentration of ammonia of all stream-water samples. At site TU1, nutrient concentrations and loads were larger during stormflow conditions than during base flow. Indicator bacteria densities and loads were larger during stormflow conditions, indicating that nonpoint sources are the largest contributors of indicator bacteria upstream from site TU1.

Pesticide and wastewater compounds were detected more often and at larger concentrations in base-flow samples from the Turkey Creek WWTF (site TU3) than in stormflow samples. Samples from site TU3 had among the most detections of wastewater and pharmaceutical compounds of all Johnson County stream-water samples. Site TU3 had the largest nonylphenol-diethoxylate concentration $(63 \mu \mathrm{g} / \mathrm{L})$ of all streamwater samples, and site TU1 had the largest NPEO2 concentration of all stormflow samples $(51 \mu \mathrm{g} / \mathrm{L})$. Many wastewater and pharmaceutical compounds, including 3-beta-coprostanol (5 $\mu \mathrm{g} / \mathrm{L})$, 4-nonylphenol (17 $\mu \mathrm{g} / \mathrm{L})$, DEET $(3.7 \mu \mathrm{g} / \mathrm{L})$, tri(2-butoxyethyl) phosphate $(27 \mu \mathrm{g} / \mathrm{L})$, acetaminophen $(2.9 \mu \mathrm{g} / \mathrm{L})$, cotinine $(0.74 \mu \mathrm{g} / \mathrm{L})$, and 1,7 dimethylxanthine (caffeine metabolite, $5.1 \mu \mathrm{g} / \mathrm{L}$ ), were detected at among the largest concentrations in base-flow samples from site TU3. The trickling-filter secondary treatment process at the Turkey Creek WWTF likely is one contributing factor to less effective removal of wastewater and pharmaceutical compounds in samples from site TU3.

The streambed-sediment sample from site TU1 exceeded six of nine USEPA TELs for trace elements, had among the smallest nutrient and indicator bacteria concentrations, and had among the smallest total wastewater compound concentrations. Total chlordane $(5 \mu \mathrm{g} / \mathrm{kg})$ also was detected in this sample. PAHs were detected in the Turkey Creek streambed-sediment sample, likely due to urban runoff containing contaminants from automobile emissions. The sample from site TU1 had 
among the most detections of pharmaceutical compounds (six) but among the smallest total concentrations $(6.1 \mu \mathrm{g} / \mathrm{kg})$ of streambed-sediment sites upstream from WWTF discharges.

\section{Summary and Conclusions}

Stream-water and sediment-quality samples were collected by the U.S. Geological Survey in 12 watersheds in Johnson County, northeastern Kansas, to determine the effects of nonpoint and selected point contaminant sources on streamwater quality and their relation to varying land use. The streams studied were located in urban areas of the county (Brush, Dykes Branch, Indian, Tomahawk, and Turkey Creeks), developing areas of the county (Blue River and Mill Creek), and in more rural areas of the county (Big Bull, Captain, Cedar, Kill, and Little Bull Creeks). Data were evaluated to determine the distribution of potential water-quality contaminants relative to potential contaminant sources and varying land-use characteristics.

Wastewater-treatment facility (WWTF) discharges proved to be an important factor in the occurrence of many constituents in Johnson County base-flow samples. Discharge from these facilities comprised greater than 50 percent of streamflow at the farthest downstream sampling site in six of the seven watersheds with WWTF sites during base-flow conditions. Samples from sites at or just downstream from WWTF discharges often contained the largest concentrations of dissolved solids, nutrients, pesticides, and wastewater-indicator and prescription and nonprescription pharmaceutical compounds in Johnson County streams. Concentrations of most constituents decreased in samples collected farther downstream from WWTF discharges where the constituents were either assimilated by stream biota, adsorbed onto streambed sediment, or degraded to metabolites. During base-flow conditions, sites upstream from WWTF discharges had significantly larger fecal coliform and E. coli densities than downstream sites. Stormflow samples had the largest suspended-sediment concentrations and indicator bacteria densities as well as the largest loads of these constituents. Nutrient concentrations and loads were generally larger with increasing flow at stormflow sampling sites. Stormflow samples also had some of the largest concentrations and loads of pesticide compounds in the spring after pesticide application. Stormflow samples consistently had the largest loads of suspended sediment, nutrients, indicator bacteria, and nonylphenoldiethoxylate, indicating the presence of nonpoint sources of these constituents.

During base-flow conditions, the effectiveness of wastewater-treatment processes appears to be an important factor in the occurrence and concentration of nutrients, pesticides, and wastewater-indicator compounds from point-source discharges. Samples from two of three trickling-filter WWTFs exceeded Kansas Department of Health and Environment $\mathrm{pH}$ - and temperature-dependent chronic aquatic-life criteria for ammonia when early-life stages of fish are present. Samples from the three trickling-filter WWTFs also had the most detections and often the largest concentrations of wastewater-indicator compounds of all Johnson County stream-water samples. Many wastewater-indicator compounds in Johnson County base-flow samples (mostly from trickling-filter discharges) exceeded maximum concentrations found in a national reconnaissance study; however, the compounds caffeine (stimulant), nonylphenol-diethoxylate (detergent surfactant), and tris(2-butoxyethyl) phosphate (flame retardant) also exceeded maximum concentrations measured in a Minnesota study, which included samples of raw sewage, landfill and animal lagoon leachate, and WWTF discharges.

Land use was another important factor affecting the occurrence and magnitude of many water-quality constituents. Upstream from WWTFs, samples from sites in urban watersheds, Brush Creek, Dykes Branch, Indian Creek, Tomahawk Creek, and Turkey Creek, often had larger concentrations of indicator bacteria and wastewater-indicator compounds during base-flow conditions than did samples from sites in nonurban areas. However, base-flow conditions at upstream sites contributed a relatively small part of the total load of these constituents compared to WWTF discharges.

Differences among sites were more difficult to identify in stormflow samples due to limited data. Suspended-sediment concentrations, nutrients, and indicator bacteria densities had statistically significant positive trends with streamflow at all stormflow sites; however, indicator bacteria densities were more significantly related to increasing suspended-sediment concentrations. Stormflow samples in urban watersheds also were shown to contain detectable concentrations of select wastewater-indicator compounds $(9,10$-anthraquinone, caffeine, carbazole, DEET, nonylphenol-diethoxylate, tris(2-butoxyethyl) phosphate). These concentrations indicate that some wastewater compounds also have nonpoint sources, primarily in urban watersheds.

Seasonality affected the concentrations of many contaminants. Selected samples collected during the winter and early spring were affected by road-salt application and contained the largest dissolved-solids concentrations, including one sample from an urban site that had a chloride concentration larger than the Kansas Department of Health and Environment acute aquatic-life criterion. Stormflow samples collected at more rural sites during the spring and summer after pesticide application had the largest pesticide concentrations.

The effects of WWTF discharges and varying land use were observable in streambed-sediment samples. Trace elements, total chlordane, total dichloro-diphenyl-trichloroethane (DDT), polycyclic aromatic hydrocarbons (PAHs), and some wastewater-indicator compounds were detected at the largest concentrations in samples from urban sites both upstream and downstream from WWTF discharges. Organic carbon, nutrients, silver, and total wastewater and pharmaceutical compounds were detected in the largest concentrations in samples collected downstream from WWTF discharges.

During base-flow conditions, point-source contributions from WWTFs were the most substantial source of water-quality contaminants in Johnson County streams; however, 
contaminant concentrations decreased substantially with increasing distance from WWTF discharges. Stormflow samples had larger concentrations of some constituents and larger instantaneous loads of most detected constituents. Sources from different land uses affected the type and amount of waterquality constituents in Johnson County streams. Samples from streams draining urban areas had increased densities of indicator bacteria and larger concentrations of selected wastewaterindicator compounds. Samples from nonurban streams had the largest concentrations of pesticide compounds during spring runoff.

Information from this study can be used to help define current (2005) water-quality conditions in Johnson County streams and to help identify source areas of water-quality contamination.

\section{References Cited}

Agency for Toxic Substances and Disease Registry, 1995a, Division of Toxicology FAQs, PAHs: Information available on the Web, accessed August 13, 2004, at http://www.atsdr.cdc.gov/tfacts69.pdf

Agency for Toxic Substances and Disease Registry, 1995b, Division of Toxicology FAQs, cresols: Information available on the Web, accessed August 13, 2004, at http://www.atsdr.cdc.gov/tfacts34.html

Agency for Toxic Substances and Disease Registry, 2002, Division of Toxicology FAQs, aldrin and dieldrin: Information available on the Web, accessed August 5, 2004, at http://www.atsdr.cdc.gov/tfacts1.pdf

American Public Health Association, American Water Works Association, and Water Environment Federation, 1995, Standard methods for the examination of water and wastewater (19th ed.): Washington, D.C., American Public Health Association, $905 \mathrm{p}$.

Benedik, M., Gibbs, P., Riddle, R., and Wilson, R. 1998, Microbial denitrogenation of fossil fuels: Trends in Biotechnology, v. 16 , p. 390-395.

Bitsch, N., Dudas, C., Korner, W., Failing, K., Biselli, S., Rimkus, G., and Brunn, H., 2002, Estrogenic activity of musk fragrances detected by the E-screen assay using human mcf-7 cells: Archives of Environmental Contamination and Toxicology, v. 43, p. 257-264.

Blevins, D.W., 1986, Quality of stormwater runoff in the Blue River Basin, Missouri and Kansas, July-October 1981 and April-July 1982: U.S. Geological Survey Water-Resources Investigations Report 84-4226, $131 \mathrm{p}$.

Buchanan, T.J., and Somers, W.P., 1969, Discharge measurements at gaging stations: U.S. Geological Survey Techniques of Water-Resources Investigations, book 3, chap. A8, 65 p.

Buerge, J., Ignaz, P., Thomas, J., Müller, M.D., and Buser, H., 2003, Caffeine, an anthropogenic marker for wastewater contamination of surface waters: Environmental Science and Technology, v. 37, p. 691-700.
Cahill, J.D., Furlong, E.T., Burkhardt, M.R, Kolpin, D., and Anderson, L.G., 2004, Determination of pharmaceutical compounds in surface- and ground-water samples by solidphase extraction and high-performance liquid chromatography-electrospray ionization mass spectrometry: Journal of Chromatography A, v. 1041, p. 171-180.

Capel, P.D., and Larson, S.J., 2001, Effect of scale on behavior of atrazine in surface waters: Environmental Science and Technology, v. 35, no. 4, p. 648-657.

Chan, K.H., Lam, M.H.W., Poon, K.F., Yeung, H.Y., and Chiu, K.T., 1998, Application of sedimentary fecal stanols and sterols in tracing sewage pollution in coastal waters: Water Research, v. 32, no. 1, p. 225-235.

Childress, C.J.O., Foreman, W.T., Connor, B.F., and Maloney, T.J., 1999, New reporting procedures based on long-term method detection levels and some considerations for interpretations of water-quality data provided by the U.S. Geological Survey National Water-Quality Laboratory: U.S. Geological Survey Open-File Report 99-193, 19 p.

Christensen, V.G., Jian, Xiaodong, and Ziegler, A.C., 2000, Regression analysis and real-time water-quality monitoring to estimate constituent concentrations, loads, and yields in the Little Arkansas River, south-central Kansas, 1995-99: U.S. Geological Survey Water-Resources Investigations Report 00-4126, 36 p.

Cook, D.L., LaFleur, L., Parrish, A., Jones, J., and Hoy, D., 1997, Characterization of plant sterols from 22 U.S. pulp and paper mills: Water Science and Technology, v. 35, no. 2-3, p. 297-303.

Daughton, C.G., and Ternes, T.A., 1999, Pharmaceuticals and personal care products in the environment - agents of subtle change: Environmental Health Perspectives, v. 107 (supplement 6), p. 907-938.

Faires, L.M., 1993, Methods of analysis by the U.S. Geological Survey National Water-Quality Laboratory-determination of metals in water by inductively coupled plasma-mass spectrometry: U.S. Geological Survey Open-File Report 92-634, $28 \mathrm{p}$.

Fent, G., Werner, J., Moendel, M., and Kubiak, R., 2003, Fate of ${ }^{14}$ C-bisphenol A in soils: Chemosphere, v. 51, p. 735-746.

Ferguson, P.L., Iden, C.R., and Brownawell, B.J., 2001, Distribution and fate of neutral alkylphenol ethoxylate metabolites in a sewage-impacted estuary: Environmental Science and Technology, v. 35, no. 12, p. 2428-2435.

Fisher, B.E., 1998, Scents and sensitivity: Environmental Health Perspectives, v. 106, no. 12, information available on the Web, accessed August 5, 2004, at http://ehp.niehs.nih.gov/docs/1998/106-12/focus.html Fishman, M.J., ed., 1993, Methods of analysis by the U.S. Geological Survey National Water-Quality Laboratorydetermination of inorganic and organic constituents in water and fluvial sediments: U.S. Geological Survey Open-File Report 93-125, 217 p. 
Fishman, M.J., and Friedman, L.C., 1989, Methods for determination of inorganic substances in water and fluvial sediments: U.S. Geological Survey Techniques of WaterResources Investigations, book 5, chap. A1, 545 p.

Foreman, W.T., Connor, B.F., Furlong, E.T., Vaught, D.G., and Merten, L.M., 1995, Methods of analysis by the U.S. Geological Survey National Water-Quality Laboratory-determination of organochlorine pesticides and polychlorinated biphenyls in bottom sediment by dual capillary- column gas chromatography with electron-capture detection: U.S. Geological Survey Open-File Report 95-140, 78 p.

Francy, D.S., and Darner, R.A., 1998, Factors affecting Escherichia coli concentrations at Lake Erie public bathing beaches: U.S. Geological Survey Water-Resources Investigations Report 98-4241, 48 p.

Furlong, E.T., Kinney, C.A., Ferrer, I., Werner, S.L., Ratterman, G., and Cahill, J.D., 2005, Determination of human health pharmaceuticals and antimicrobials in terrestrial and aquatic solids: U.S. Geological Survey Scientific Investigations Report, in press.

Giger, W., Brunner, P.H., and Schaffner, C., 1984, 4-Nonylphenol in sewage sludge-accumulation of toxic metabolites from non-ionic surfactants: Science, v. 225, p. 623-625.

Gu, J., Yanzhen, F., and Hanchang, S., 2002, Relationship between structures of substituted indolic compounds and their degradation by marine anaerobic microorganisms: Marine Pollution Bulletin, v. 45, p. 379-384.

Guy, H.P., 1969, Laboratory theory and methods for sediment analysis: U.S. Geological Survey Techniques of WaterResources Investigations, book 5, chap. C1, $58 \mathrm{p}$.

Heberer, T., These, A., and Grosch, U.A., 2001, Occurrence and fate of synthetic musks in the aquatic system of urban areas-polycyclic and nitro musks as environmental pollutants in surface waters, sediments, and aquatic biota, pharmaceuticals and personal care products in the environment: Washington, D.C., American Chemical Society, p. 143-155.

Helsel, D.R., and Hirsch, R.M., 1992, Statistical methods in water resources: Amsterdam, Elsevier Science Publishers, $522 \mathrm{p}$.

Hem, J.D., 1992, Study and interpretation of the chemical characteristics of natural water (3rd ed.): U.S. Geological Survey Water-Supply Paper 2254, 263 p.

Hodel, D.R., and Chang, A.C., 2004, Trace elements and urban gardens: Information available on the Web, accessed December 21, 2004, at http://celosangeles.ucdavis.edu/ envhort/trace_elements.html

Horowitz, A.J., 1991, A primer on trace metal-sediment chemistry (2nd ed): Ann Arbor, Michigan, Lewis Publishing Company, $136 \mathrm{p}$.

Horowitz, A.J., Elrick, K.A., and Smith, J.J., 2001, Estimating suspended sediment and trace element fluxes in large river basins-methodological considerations as applied to the NASQAN programme: Hydrological Processes, v. 15, no. 7, p. $1169-1208$.

Howell, J.M., Coyne, M.S., and Cornelius, P.L, 1996, Effect of sediment particle size and temperature on fecal bacteria mor- tality rates and the fecal coliform/fecal streptococci ratio: Journal of Environmental Quality, v. 21, p. 1216-1220.

Isobe, K.O., Tarao, M., Zakaria, M.P., Chiem, N.H., Minh, L.Y., and Takada, H., 2002, Quantitative application of fecal sterols using gas chromatography-mass spectrometry to investigate fecal pollution in tropical waters-Western Malaysia and Mekong Delta, Vietnam: Environmental Science and Technology, v. 36, no. 21, p. 4497-4507.

Jobling, S., Sheahan, D., Osborne, J.A., Matthiessen, P., and Sumpter, J.P., 1996, Inhibition of testicular growth in rainbow trout (Oncorhynchus mykiss) exposed to estrogenic alkylphenolic chemicals: Environmental Toxicology and Chemistry, v. 15, p. 194-202.

Jobling, S., and Sumpter, J.P., 1993, Detergent components in sewage effluent are weakly oestrogenic to fish — an in vitro study using rainbow trout (Oncorhynchusmykiss): Aquatic Toxicology, v. 27, p. 661-672.

Kansas Department of Health and Environment, 2002, Kansas register, article 28-16-28e, Surface water quality standards: Topeka, Kansas, Secretary of State, 22 p.

Kansas Department of Health and Environment, 2004a, Kansas register, article 28-15-201, Public notification of drinking water standards: Topeka, Kansas, Secretary of State, 19 p.

Kansas Department of Health and Environment, 2004b, Total maximum daily load designations: Information available on the Web, accessed June 15, 2004, at http://www.kdhe.state.ks.us/tmdl/

Kansas Department of Health and Environment, 2004c, Kansas register, article 28-16-28e, Surface water quality standards: Topeka, Kansas, Secretary of State, 10 p.

Kiely, T., Donaldson, D., and Grube, A., 2004, Pesticides industry sales and usage, 2000 and 2001 market estimates: Information available on the Web, accessed January 24, 2005, at http://www.epa.gov/oppbead1/ pestsales/01pestsales/market_estimates2001.pdf

Kolpin, D.W., Furlong, E.T., Meyer, M.T., Thurman, E.M., Zaugg, S.D., Barber, L.B., and Buxton, H.T., 2002, Pharmaceuticals, hormones, and other organic wastewater contaminants in U.S. streams, 1999-2000-a national reconnaissance: Environmental Science and Technology, v. 36, no. 6, p. 1202-1211.

Lee, G.F., and Sears, R.I., 1985, Kansas Clean Lakes Program, Lake Olathe: prepared for the city of Olathe, Kansas, various pagination.

Lee, K.E., Barber, L.T., Furlong, E.T., Cahill, J.D., Kolpin, D.W., Meyer, M.T., and Zaugg, S.D., 2004, Presence and distribution of organic wastewater compounds in wastewater, surface, ground, and drinking waters, Minnesota, 2000-02: U.S. Geological Survey Scientific Investigations Report 2004-5138, 47 p.

Leeming, R., Ball, A., Ashbolt, N., and Nichols, P., 1996, Using faecal sterols from humans and animals to distinguish fecal pollution in receiving waters: Water Research, v. 30, p. 2893-2900. 
Lewis, M.E., and Zaugg, S.D., 2003, National field manual for the collection of water-quality data: U.S. Geological Survey Techniques of Water-Resources Investigations, book 9, chap. A5, 9 p.

Maidment, D.R., 1993, Handbook of hydrology: New York, McGraw-Hill, Inc., variously paged.

Martin, J.D., Crawford, C.G., and Larson, S.J., 2003, Pesticides in streams - summary statistics, preliminary results from cycle I of the National Water-Quality Assessment Program (NAWQA), 1992-2001: Information available on the Web, accessed October 22, 2004, at http://ca.water.usgs.gov/ pnsp/pestsw/Pest-SW_2001_Text.html

Masters, G.M., 1991, Introduction to environmental engineering and science: Englewood Cliffs, New Jersey, Prentice Hall, p. 110-111.

Mau, D.P., 2000, Sediment deposition and selected water-quality characteristics in Cedar Lake and Lake Olathe, northeast Kansas, 2000: U.S. Geological Survey Water-Resources Investigations Report 02-4073, 71 .

Mau, D.P., Ziegler, A.C., Porter, S.D., and Pope, L.M., 2004, Surface-water-quality conditions and relation to taste-andodor occurrences in the Lake Olathe watershed, northeast Kansas, 2000-02: U.S. Geological Survey Scientific Investigations Report 2004-5047, 95 p.

McKinney, R.A., Pruell, R.J., and Burgess, R.M., 1999, Ratio of concentration of anthraquinone to anthracene in coastal marine sediments: Chemosphere, v. 38, no. 10, p. 2415-2430.

Mid-America Regional Council, 2002, Long-range forecast for the Kansas City metropolitan area, 2002: Information available on the Web, accessed December 17, 2004, at http://www.metrodataline.org/Forecasts/ 2002\%20Long\%20Range\%20Forecast.xls

Mid-America Regional Council and F.X. Browne Associates, Inc., 1983, Nationwide Urban Runoff Program-Kansas City area project-executive and technical summaries: Kansas City, Missouri, Mid-America Regional Council, 16 p.

Mitra, S., and Bianchi, T.S., 2003, A preliminary assessment of polycyclic aromatic hydrocarbon distributions in the lower Mississippi River and Gulf of Mexico: Marine Chemistry, v. 82 , no. 3-4, p. 273-288.

National Oceanic and Atmospheric Administration, 1966-98, Climatological data annual summary-east-central Kansas: Asheville, North Carolina, National Weather Service Climatic Data Center, published monthly.

O’Connor, H.G., 1971, Johnson County geohydrology: Kansas Geological Survey, information available on the Web, accessed July 20, 2004, at http://www.kgs.ku.edu/ General/Geology/Johnson/index.html

Overland Park Stormwatch, 2004, Overland Park, Kansas, flood warning system: Information available on the Web, accessed at July 21, 2004, at http://www.stormwatch.com/index.asp

Phillips, P.J., Stinson, B., Zaugg, S.D., and Furlong, E.T., 2004, Concentrations of organic compounds in wastewater at five sites in New York State, 2003, in Emerging Issues Confer- ence, October 13-15, 2004, Minneapolis, Minnesota: National Ground Water Association, p. 73.

Plinsky, R.O., Zimmerman, J.L., Dickey, H.P., Jorgensen, G.N., Fenwick, R.W., and Roth, W.E, 1975, Soil survey of Johnson County, Kansas: U.S. Department of Agriculture, Soil Conservation Service, 93 p.

Pope, L.M., and Putnam, J.E., 1997, Effects of urbanization on water quality in the Kansas River, Shunganunga Creek Basin and Soldier Creek, Topeka, Kansas, October 1993 through September 1995: U.S. Geological Survey Water-Resources Investigations Report 97-4045, 84 p.

Putnam, J.E., 1997, Occurrence of phosphorus, other nutrients, and triazine herbicides in water from the Hillsdale Lake Basin, northeast Kansas, May 1994 through May 1995: U.S. Geological Survey Water-Resources Investigations Report 97-4019, 66 p.

Quéméneur, M., and Marty, Y., 1992, Sewage influence in a macrotidal estuary - fatty acids and sterol distributions: Estuarine, Coastal and Shelf Science, v. 34, p. 347-363.

Quéméneur, M., and Marty, Y., 1994, Fatty acids and sterols in domestic wastewaters: Water Research, v. 28, p. 1217-1226.

Rasmussen, P.P., and Ziegler, A.C, 2003, Comparison and continuous estimates of fecal coliform and Escherichia coli bacteria in selected Kansas streams, May 1999 through April 2002: U.S. Geological Survey Water-Resources Investigations Report 03-4056, 80 p.

Routedge, E.J., and Sumpter, J.P., 1997, Structural features of alkylphenolic chemicals associated with estrogenic activity: Journal of Biological Inorganic Chemistry, v. 272, p. 3280 3288.

Runnels, R., and Schleicher, J., 1956, Chemical composition of eastern Kansas limestones: Kansas Geological Survey Bulletin 119, pt. 3, p. 81-103.

Sabaliunas, D., Webb, S.F., Hauk, A., Jacob, M., and Eckhoff, W.S., 2003, Environmental fate of triclosan in the River Aire Basin, UK: Water Research, v. 37, p. 3145-3154.

Schoewe, W.H., 1949, The geography of Kansas: Transactions of the Kansas Academy of Science, v. 52, no. 3, p. 261-333. Schottler, S.P., Eisenreich, S.J., and Capel, P.D., 1994, Atrazine, alachlor and cyanazine in a large agricultural river system: Environmental Science and Technology, v. 28, p. 1079-1089.

Seiler, R.L., Zaugg, S.D., Thomas, J.M., and Howcroft, D.L.,1999, Caffeine and pharmaceuticals as indicators wastewater contamination in wells: Ground Water, v. 37, no. 3, p. 405-410.

Shakibaie, M.R., Kapadnis, B.P., Dhakephalker, P., and Chopade, B.A., 1999, Removal of silver from photographic wastewater effluent using Acinetobacter baumannii BL54: Canadian Journal of Microbiology, v. 45, no. 12, p. 9951000.

Soto, A.M., Chung, K.L., and Sonnenschein, C., 1994, The pesticides endosulfan, toxaphene, and dieldrin have estrogenic effects on human estrogen-sensitive cells: Environmental Health Perspectives, v. 102, no. 4, p. 380-383. 
Soto, A.M., Justica, H., Wray, J.W., and Sonnenschein, C., 1991, p-Nonyl-phenol—an estrogenic xenobiotic released from "modified" plystyrene: Environmental Health Perspectives, v. 92, p. 167-173.

U.S. Census Bureau, 2004, Kansas population of county by decennial census, 1900-90: Information available on the Web, accessed July 15, 2004, at http://quickfacts.census.gov/qfd/states/20/20091.html, http://www.census.gov/population/cencounts/ks190090.txt, and http://www.census.gov/population/estimates/ metro-city//03mfips.txt

U.S. Environmental Protection Agency, 1972, DDT ban takes effect: Information available on the Web, accessed August 9, 2004, at http://www.epa.gov/history/topics/ddt/01.htm

U.S. Environmental Protection Agency,1986, Ambient water quality for bacteria - 1986: Information available on the Web, accessed August 4, 2004, at http://www.epa.gov/ waterscience/beaches/1986crit.pdf

U.S. Environmental Protection Agency, 1998a, DEET facts: U.S. Environmental Protection Agency Report EPA-738-F95-010, information available on the Web, accessed June 21, 2004, at http://www.epa.gov/oppsrrd1/REDs/ factsheets/0002fact.pdf

U.S. Environmental Protection Agency 1998b, The incidence and severity of sediment contamination in surface waters of the United States, volume 1-national sediment survey: U.S. Environmental Protection Agency Report 823-R-97-006, various pagination.

U.S. Environmental Protection Agency, 1999, 1999 Update of ambient water quality criteria for ammonia: Information available on the Web, accessed May 2, 2005, at http://www.epa.gov/waterscience/standards/ ammonia/99update.pdf

U.S. Environmental Protection Agency, 2000a, Methods for measuring the toxicity and bioaccumulation of sedimentassociated contaminants with freshwater invertebrates (2nd ed.): Information available on the Web, accessed October 26, 2004, at http://www.epa.gov/waterscience/cs/ freshmanual.pdf

U.S. Environmental Protection Agency, 2000b, Ambient water quality criteria recommendations for rivers and streams in nutrient Ecoregion IX: Information available on the Web, accessed October 14, 2004, at http://www.epa.gov/ waterscience/criteria/nutrient/ecoregions/rivers/rivers_9.pdf

U.S. Environmental Protection Agency, 2002, List of drinking water contaminants and their MCLs: Information available on the Web, accessed October 18, 2004, at http://www.epa.gov/safewater/mcl.html\#mcls

U.S. Environmental Protection Agency, 2003, Ambient aquatic life water quality criteria for nonylphenol-draft: Information available on the Web, accessed August 9, 2004, at http://www.epa.gov/waterscience/criteria/ nonylphenol/draft-nonylphenol.pdf

U.S. Environmental Protection Agency 2004a, Consumer fact sheet on chlordane: Information available on the Web, accessed August 9, 2004, at http://www.epa.gov/ safewater/contaminants/dw_contamfs/chlordan.html

U.S. Environmental Protection Agency 2004b, Monitoring and assessing water quality, chapter 5.11, fecal bacteria: Information available on the Web, accessed November 4, 2004, at http://www.epa.gov/OWOW/monitoring/ volunteer/stream/vms511.html

U.S. Environmental Protection Agency, 2005a, National Pollutant Discharge Information System (NPDES): Information available on the Web, accessed April 6, 2005, at http://cfpub.epa.gov/npdes/

U.S. Environmental Protection Agency, 2005b, Anthraquinone fact sheet: Information available on the Web, accessed May 3, 2005, at http://www.epa.gov/pesticides/ biopesticides/ingredients/factsheets/factsheet_122701.htm

U.S. Geological Survey, 2004, National Water-Quality Assessment (NAWQA) Program retrospective database for nutrients in groundwater and surface water: Information available on the Web, accessed December 20, 2004, at http://water.usgs.gov/nawqa/nutrients/datasets/retro.data.sw

Van Donsel, D., and Geldreich, E., 1971, Relationship of salmonellae to fecal coliforms in bottom sediments: Water Resources, v. 5, p. 1079-1087.

Van Metre, P.C., Mahler, B.J., and Furlong, E.T., 2000, Urban sprawl leaves its PAH signature: Environmental Science and Technology, v. 34, no. 19, p. 4064-4070.

WaterOne, 2004, Water District No. 1 of Johnson Countydrinking water quality report 2004: Information available on the Web, accessed October, 20, 2004, at http://www.waterone.org/Adobe\%20Files/ 2004\%20Water\%20Quality\%20Report_1.pdf

Weete, J.D., 1976, Algal and fungal waxes-chemistry and biochemistry of natural waxes: Amsterdam, Elsevier, p. 349-418.

White, R., Jobling, S., Hoarde, S.A., Sumpter, J.P., and Parker, M.G., 1994, Environmentally persistent alkylphenolic compounds are estrogenic: Endocrinology, v. 135, no. 1, p. $175-182$.

Wilde, F.O., Radtke, D.B., Gibs, J., and Iwatsubo, R.T., eds., 1998, National field manual for the collection of waterquality data: U.S. Geological Survey Techniques of WaterResources Investigations, book 9, chap. A7, 49 p.

Wilkison, D.H., Armstrong, D.J., and Blevins, D.W., 2002, Effects of combined sewer overflows on water quality in the Blue River Basin, Kansas City, Missouri and Kansas, June 1998-October 2000: U.S. Geological Survey WaterResources Investigations Report 02-4107, 162 p.

World Health Organization, 1998, Concise international chemical assessment document on limonene no. 5, in World Health Organization, International Programme on Chemical Safety, Geneva: Information available on the Web, accessed August 13, 2004, at http://www.inchem.org/ documents/cicads/cicads/cicad05.htm 
World Health Organization, 2000, Environmental health criteria for flame retardants - tris(2-butoxyethyl) phosphate, tris(2-ethylhexyl) phosphate, tetrakis-(hydroxymethyl) phosphonium salts: Geneva, Environmental Health Criteria \# 218, information available on the Web, accessed August 13, 2004, at http://www.inchem.org/documents/ehc/ehc/ ehc218.htm

Ying, G., Williams, B., and Kookana, R., 2002, Environmental fate of alkylphenols and alkylphenol ethoxylates-a review: Environment International, v. 28, p. 215-226.

Zaugg, S.D., Sandstrom, M.W., Smith, S.G., and Fehlberg, K.M., 1995, Methods of analysis by the U.S. Geological Survey National Water-Quality Laboratory-determination of pesticides in water by $\mathrm{C}$ - 18 solid-phase extraction and capillary-column gas chromatography/mass spectrometry with selected-ion monitoring: U.S. Geological Survey Open-File Report 95-181, 60 p.

Zaugg, S.D., Smith, S.G., Schroeder, M.P., Barber, L.B., and Burkhardt, M.R., 2002, Methods of analysis by the U.S. Geological Survey National Water-Quality Laboratorydetermination of wastewater compounds by polystyrenedivinylbenzene solid-phase extraction and capillary-column gas chromatography/mass spectrometry: U.S. Geological Survey Water-Resources Investigations Report 01-4186, $37 \mathrm{p}$.

Zhou, J.L., and Liu, Y.P., 2000, Kinetics and equilibria of the interactions between diethylhexl phthalate and sediment particles in simulated estuarine systems: Marine Chemistry, v. 71 , no. $1-2$, p. $165-176$. 